University of Tennessee Health Science Center

UTHSC Digital Commons

\title{
The Long-Term Teratogenic Effect of Prenatal Alcohol Exposure on the Somatosensory and Motor Cortex of Rats
}

Tyson Chappell

University of Tennessee Health Science Center

Follow this and additional works at: https://dc.uthsc.edu/dissertations

Part of the Nervous System Commons

\section{Recommended Citation}

Chappell, Tyson, "The Long-Term Teratogenic Effect of Prenatal Alcohol Exposure on the Somatosensory and Motor Cortex of Rats" (2007). Theses and Dissertations (ETD). Paper 41. http://dx.doi.org/10.21007/ etd.cghs.2007.0048.

This Dissertation is brought to you for free and open access by the College of Graduate Health Sciences at UTHSC Digital Commons. It has been accepted for inclusion in Theses and Dissertations (ETD) by an authorized administrator of UTHSC Digital Commons. For more information, please contact jwelch30@uthsc.edu. 


\title{
The Long-Term Teratogenic Effect of Prenatal Alcohol Exposure on the Somatosensory and Motor Cortex of Rats
}

\begin{abstract}
The primary goal of this dissertation was to examine the long-term effect of prenatal alcohol exposure (PAE) on the primary somatosensory $(\mathrm{SI})$ and primary motor cortex $(\mathrm{MI})$. For over 30 years, PAE has been shown to produce a triad of symptoms (growth deficits, craniofacial dysmorphologies and central nervous system disruptions) diagnosed as fetal alcohol syndrome (FAS) in children and adults. Since low to moderate levels of prenatal alcohol exposure are less likely to produce FAS symptoms in children, the term "PAE" in this dissertation typically refers to heavy/abusive/chronic levels of prenatal alcohol exposure (in humans this equates to $5+$ drinks/per occasion/1+ times per week) administered in a chronic binge-type fashion having a high potential to cause FAS. However, it must be pointed out that the most important factor in studies dealing with PAE in humans or animals is the peak blood alcohol level (BAL) and next are the temporal and spatial parameters dealing with high BALs. It is evident that children with FAS frequently show major deficits in information processing, which is one of the major functions of the sensorimotor system. Two major components of the sensorimotor system are the cortical input to layer IV primary sensory cortex (SI) and output from layers $\mathrm{V}$ and $\mathrm{VI}$ primary motor cortex (MI). Therefore, the focus of this dissertation is on PAE-related anatomical and physiological deficits in SI and MI that are likely to play a role in the multiple sensorimotor, behavioral, and learning disruptions seen in children with FAS.

The similarities in rodent and human cortical organization make rodents excellent models for elucidating the influence of PAE on the sensorimotor system. Of particular note is the highly organized human and rodent sensorimotor system with the sensory input and motor output arranged into structural and functional columns. However, one difference between humans and rodents is that the latter receives its sensory information in layer IV cortex into cell aggregates termed "barrels," whereas humans have no such specific cortical structures per se. Each barrel in rodent cortex is spatially related to sensory receptors that innervate specific regions of the body surface, thus barrels produce a cortical somatic map. The longest studied and most easily identifiable barrel region is the posterior medial barrel subfield (PMBSF), representing mystacial vibrissae of the rodent. A second highly studied region of the barrel cortex is the forepaw barrel subfield (FBS), representing the forepaw.
\end{abstract}

One hallmark feature of PAE is the behavioral disruptions seen in fine motor control. These deficits may be due to disruptions in both $\mathrm{SI}$ and $\mathrm{MI}$. Like SI, MI is also well organized into specific cortical regions associated with eliciting motor responses. Because SI and $\mathrm{MI}$ are highly organized, these cortical regions are very useful for studying the effect of prolonged PAE-related sensorimotor disruptions.

The primary goal of this dissertation was to examine the long-term consequences of PAE on SI and MI in 6-week-old (juvenile) or 7-month-old (adult) rats. To accomplish this goal, four major experiments were carried out utilizing a binge-type alcohol exposure paradigm on pregnant rat dams followed by an examination of the PAE pups as well as untreated control and nutritional control pups at six weeks and seven months of age.

Experiment 1: We tested the hypothesis (Chapter 2) that PAE would reduce the PMBSF area in juvenile and adult rats. Results indicated that PAE significantly reduced the PMBSF area, the total PMBSF barrel area, and interbarrel septa area in PAE juveniles and adult rats. Furthermore, PAE effects were asymmetrical across the PMBSF, as the areas of posterior barrels were less reduced than anterior barrel areas. By combining barrels into lateral-to-medial running "arcs" and calculating the area as a percent difference from respective chowfed (CF) or pairfed (PF) arcs, results suggested a split in the PMBSF between a more reduced anterior region and a less reduced posterior region. The areas of anterior arcs of 
PMBSF were always more reduced than the areas of posterior arcs. However, a similar asymmetric pattern was not identified in anterior to posterior running rows.

Experiment 2: The goal of Experiment 2 (Chapter 2) was to test the hypothesis that the rostrocaudal asymmetry identified in Experiment 1 was due, in part, to differential sensory experience across the mystacial pad. It is well established that numerous peripheral and CNS asymmetries already occur by way of variations in mystacial pad vibrissae lengths, base widths, vibrissae behavior, peripheral innervation patterns, and metabolic activity levels within cortical PMBSF barrels. It was hypothesized that trimming the mystacial pad would eliminate any differences between posterior and anterior vibrissae, in turn affecting the vibrissae cortical barrel representations and subsequent PMBSF asymmetries. To test this hypothesis, unilateral mystacial vibrissae were trimmed in $\mathrm{EtOH}$ and $\mathrm{CF}$ rats from postnatal day five (PD5) to PD42. EtOH rats sacrificed on PD42 had their individual PMBSF area and individual PMBSF barrel areas measured. These areas were then compared to respective PMBSF and barrel areas of the ipsilateral cortex from a CF group that had also undergone vibrissae trimming, and the percent change was calculated. Results indicated that vibrissae trimming did not affect the asymmetry identified in Chapter 2.

Experiment 3: We tested the hypothesis that PAE would significantly reduce the area of the FBS morphology and the area of the peripheral glabrous forepaw (Chapter 3). The FBS morphology and peripheral glabrous forepaw areas were significantly reduced in juvenile rats following PAE.

Experiment 4: This experiment was carried out to test the hypothesis that PAE would reduce the physiological/functional forepaw representation and also delay evoked response latency between periphery and SI (Chapter 4). Using carbon-fiber electrodes inserted into layer IV cortex, the physiological forepaw representation was mapped by lightly tapping the forepaw periphery (i.e., digits, or pads) with a dull insect pin and recording forepaw receptive fields in the cortex. PAE significantly reduced the representation of the entire glabrous forepaw surface as well as the physiological forepaw representation.

Experiment 5: We tested the hypothesis that PAE would reduce the size of the primary motor cortex (MI), specifically those regions responsible for contralateral vibrissae and forepaw movement (Chapter 5). Results indicated that PAE significantly reduced $\mathrm{MI}$ areas for mystacial vibrissae and forepaw motor cortex.

Results from Experiments 1 to 5 reinforce the hypothesis that PAE has a long-term effect on the sensorimotor system and provides new information on the effect of PAE on MI. The sensorimotor system is important for processing peripheral information and performing planned motor movements. Therefore, deficits in this system may be among the underlying mechanisms for reported clinical symptoms, such as the disturbances in balance, posture, fine motor control, attention, learning, reaction time, and other information disruptions often identified in children exposed to high levels of alcohol in utero.

\section{Document Type}

Dissertation

Degree Name

Doctor of Philosophy (PhD)

\section{Program}

Anatomy and Neurobiology

\section{Research Advisor}

Robert S. Waters, Ph.D. 


\section{Keywords}

Prenatal alcohol, FASD, Cortex, Somatosensory, Motor, Barrels, Rodent, Vibrissae, PMBSF, FBS, Morphology, Physiology

\section{Subject Categories}

Anatomy | Medicine and Health Sciences | Nervous System 


\title{
THE LONG-TERM TERATOGENIC EFFECT OF PRENATAL ALCOHOL EXPOSURE ON THE SOMATOSENSORY AND MOTOR CORTEX OF RATS
}

\author{
A Dissertation \\ Presented for \\ The Graduate Studies Council \\ The University of Tennessee \\ Health Science Center
}

In Partial Fulfillment

Of the Requirements for the Degree

Doctor of Philosophy

From The University of Tennessee

By

Tyson Dow Chappell

December 2007 
Copyright (C) 2007 by Tyson Dow Chappell

All rights reserved 


\section{DEDICATION}

To my incredibly supportive wife Amber;

To my dear sweet children: Alexis Shea, Zachary William,

Garrett Jaxon and Drake Xavier;

And finally to my loving father and mother: Bill and Susan. 


\section{ACKNOWLEDGEMENTS}

Primarily I would like to thank Amber, my amazing wife, who bore the main responsibility of raising our four wonderful children throughout our time in Memphis. Amber, your love is always a shining beacon, even in the darkest of days. I thank my children (the fantastic four) who always seemed to understand when the workdays were long. Kids, your huge grins and energetic cheers of "daddy" as I returned home each day always invigorated me while reminding me of the true meaning and purpose of life. Next, I thank my parents for their incredible support throughout my life. Mom and dad your examples of hard work and your ability to enjoy life even when times are tough have always remained in my heart and mind. You taught me that life is not necessarily about reaching the goal at the end of each journey, rather enjoying the trip along the way. Mom and dad, thank you for teaching me honor, conviction, and optimism.

I am very grateful for the training I received in the Waters' lab. Thanks Bob, for the innumerable hours you spent reading, editing, and correcting my papers and for providing me with the many useful comments that indicated how to improve my writing skills. I thank Dr. Li for teaching me the many methods used in the lab, ranging from making electrodes and chemical solutions to performing craniotomy and deafferentation surgeries. Dr Li's patience, supportive words, and life philosophies were a constant strength. Cecilia thanks for clearing the path in many respects and showing me that graduating is possible. Cecilia, you're a great friend, lab-mate, and the sister I never had. Taha and David, it was great working with you both during the summer. I wish you two the very best. I also thank my committee members for their critical analysis of my 
qualifying exam and thesis/dissertation. I'd especially like to thank all my past teachers, instructors, mentors, and counselors. Without you, I could not have accomplished any of this. Finally, I thank NIH for the funding and support of these projects over the years with the grant awarded to Robert Waters, PhD (P.I.) and Andrea Elberger, PhD (co-P.I.), grant\# AA-013437-01. 


\begin{abstract}
The primary goal of this dissertation was to examine the long-term effect of prenatal alcohol exposure (PAE) on the primary somatosensory (SI) and primary motor cortex (MI). For over 30 years, PAE has been shown to produce a triad of symptoms (growth deficits, craniofacial dysmorphologies and central nervous system disruptions) diagnosed as fetal alcohol syndrome (FAS) in children and adults. Since low to moderate levels of prenatal alcohol exposure are less likely to produce FAS symptoms in children, the term "PAE" in this dissertation typically refers to heavy/abusive/chronic levels of prenatal alcohol exposure (in humans this equates to $5+$ drinks/per occasion/1+ times per week) administered in a chronic binge-type fashion having a high potential to cause FAS. However, it must be pointed out that the most important factor in studies dealing with PAE in humans or animals is the peak blood alcohol level (BAL) and next are the temporal and spatial parameters dealing with high BALs. It is evident that children with FAS frequently show major deficits in information processing, which is one of the major functions of the sensorimotor system. Two major components of the sensorimotor system are the cortical input to layer IV primary sensory cortex (SI) and output from layers V and VI primary motor cortex (MI). Therefore, the focus of this dissertation is on PAErelated anatomical and physiological deficits in SI and MI that are likely to play a role in the multiple sensorimotor, behavioral, and learning disruptions seen in children with FAS.
\end{abstract}

The similarities in rodent and human cortical organization make rodents excellent models for elucidating the influence of PAE on the sensorimotor system. Of particular 
note is the highly organized human and rodent sensorimotor system with the sensory input and motor output arranged into structural and functional columns. However, one difference between humans and rodents is that the latter receives its sensory information in layer IV cortex into cell aggregates termed "barrels," whereas humans have no such specific cortical structures per se. Each barrel in rodent cortex is spatially related to sensory receptors that innervate specific regions of the body surface, thus barrels produce a cortical somatic map. The longest studied and most easily identifiable barrel region is the posterior medial barrel subfield (PMBSF), representing mystacial vibrissae of the rodent. A second highly studied region of the barrel cortex is the forepaw barrel subfield (FBS), representing the forepaw.

One hallmark feature of PAE is the behavioral disruptions seen in fine motor control. These deficits may be due to disruptions in both SI and MI. Like SI, MI is also well organized into specific cortical regions associated with eliciting motor responses. Because SI and MI are highly organized, these cortical regions are very useful for studying the effect of prolonged PAE-related sensorimotor disruptions.

The primary goal of this dissertation was to examine the long-term consequences of PAE on SI and MI in 6-week-old (juvenile) or 7-month-old (adult) rats. To accomplish this goal, four major experiments were carried out utilizing a binge-type alcohol exposure paradigm on pregnant rat dams followed by an examination of the PAE pups as well as untreated control and nutritional control pups at six weeks and seven months of age.

Experiment 1: We tested the hypothesis (Chapter 2) that PAE would reduce the PMBSF area in juvenile and adult rats. Results indicated that PAE significantly reduced the PMBSF area, the total PMBSF barrel area, and interbarrel septa area in PAE juveniles 
and adult rats. Furthermore, PAE effects were asymmetrical across the PMBSF, as the areas of posterior barrels were less reduced than anterior barrel areas. By combining barrels into lateral-to-medial running "arcs" and calculating the area as a percent difference from respective chowfed (CF) or pairfed (PF) arcs, results suggested a split in the PMBSF between a more reduced anterior region and a less reduced posterior region. The areas of anterior arcs of PMBSF were always more reduced than the areas of posterior arcs. However, a similar asymmetric pattern was not identified in anterior to posterior running rows.

Experiment 2: The goal of Experiment 2 (Chapter 2) was to test the hypothesis that the rostrocaudal asymmetry identified in Experiment 1 was due, in part, to differential sensory experience across the mystacial pad. It is well established that numerous peripheral and CNS asymmetries already occur by way of variations in mystacial pad vibrissae lengths, base widths, vibrissae behavior, peripheral innervation patterns, and metabolic activity levels within cortical PMBSF barrels. It was hypothesized that trimming the mystacial pad would eliminate any differences between posterior and anterior vibrissae, in turn affecting the vibrissae cortical barrel representations and subsequent PMBSF asymmetries. To test this hypothesis, unilateral mystacial vibrissae were trimmed in EtOH and $\mathrm{CF}$ rats from postnatal day five (PD5) to PD42. EtOH rats sacrificed on PD42 had their individual PMBSF area and individual PMBSF barrel areas measured. These areas were then compared to respective PMBSF and barrel areas of the ipsilateral cortex from a CF group that had also undergone vibrissae trimming, and the percent change was calculated. Results indicated that vibrissae trimming did not affect the asymmetry identified in Chapter 2. 
Experiment 3: We tested the hypothesis that PAE would significantly reduce the area of the FBS morphology and the area of the peripheral glabrous forepaw (Chapter 3). The FBS morphology and peripheral glabrous forepaw areas were significantly reduced in juvenile rats following PAE.

Experiment 4: This experiment was carried out to test the hypothesis that PAE would reduce the physiological/functional forepaw representation and also delay evoked response latency between periphery and SI (Chapter 4). Using carbon-fiber electrodes inserted into layer IV cortex, the physiological forepaw representation was mapped by lightly tapping the forepaw periphery (i.e., digits, or pads) with a dull insect pin and recording forepaw receptive fields in the cortex. PAE significantly reduced the representation of the entire glabrous forepaw surface as well as the physiological forepaw representation.

Experiment 5: We tested the hypothesis that PAE would reduce the size of the primary motor cortex (MI), specifically those regions responsible for contralateral vibrissae and forepaw movement (Chapter 5). Results indicated that PAE significantly reduced MI areas for mystacial vibrissae and forepaw motor cortex.

Results from Experiments 1 to 5 reinforce the hypothesis that PAE has a longterm effect on the sensorimotor system and provides new information on the effect of PAE on MI. The sensorimotor system is important for processing peripheral information and performing planned motor movements. Therefore, deficits in this system may be among the underlying mechanisms for reported clinical symptoms, such as the disturbances in balance, posture, fine motor control, attention, learning, reaction time, 
and other information disruptions often identified in children exposed to high levels of alcohol in utero. 


\section{TABLE OF CONTENTS}

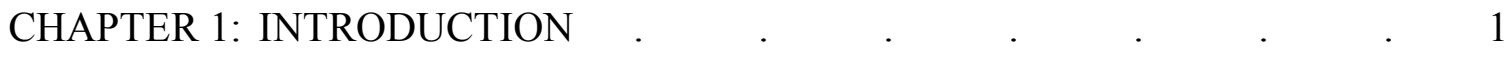

1.1 FETAL ALCOHOL SYNDROME . $\quad$. $\quad$. $\quad . \quad$. $\quad$. 1

1.2 FETAL ALCOHOL SPECTRUM DISORDER . . . . . 2

1.3 THE PRIMARY SOMATOSENSORY CORTEX . . . . . 3

1.3.1 Circuitry pathways: periphery to cortex $\quad$. $\quad . \quad$. $\quad$. 4

1.3.2 From cortical columns to cortical somatotopy $\quad$. $\quad$. $\quad$. 5

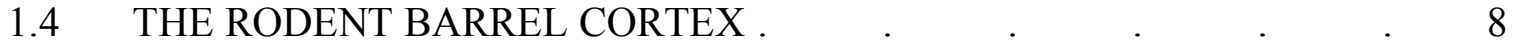

1.4.1 Barrelettes, barreloids, and barrels . $\quad . \quad$. $\quad . \quad$. 8

1.4.2 Morphological organization of layer IV barrel cortex $\quad$. $\quad$. 9

1.4.2.1 PMBSF, ALBSF, and LLBSF $\quad . \quad$ • $\quad$. $\quad . \quad 10$

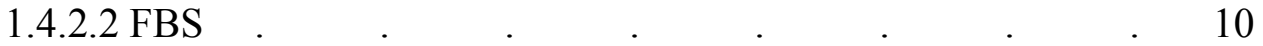

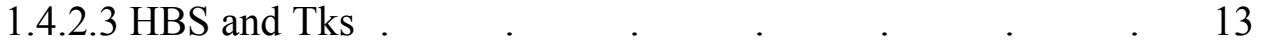

1.4.3 SI physiological forepaw representation $\quad . \quad$. $\quad . \quad$. 13

1.5 THE PRIMARY MOTOR CORTEX. . . . . . . . . $\quad$. 17

1.5.1 History of motor cortex studies _ . $\quad$. $\quad$. $\quad$. $\quad$. 17

1.5.2 Cortical cytoarchitecture related to motor maps: AGl,
AGm, and Cg1

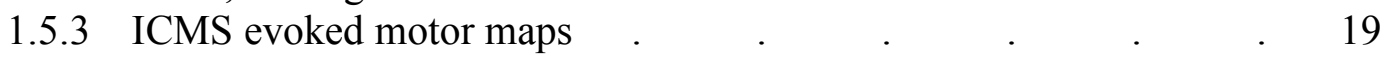

1.5.3.1 Eyelid/periocular movements respond to ICMS in Cg1 . 19

1.5.3.2 ICMS in AGm and PL evokes vibrissae movements . . 20

1.5.3.3 Forelimb responds to ICMS in AG1 . $\quad$. $\quad$. $\quad$. 21

1.6 CORTICAL DEVELOPMENT IN NORMAL RATS . . . 23

1.6.1 Pyramidal cell development . . . . . . . . 23

1.6.2 Nonpyramidal cell development . . . . . . $\quad$. 25

1.7 TERATOGENIC EFFECT OF PAE ON DEVELOPING BRAIN . 26

1.7.1 The effect of PAE on cortical neurogenesis during the first trimester (GD1 to GD12) in rodent . . . . . . 26

1.7.2 The effect of PAE on cortical neurogenesis during the second trimester (GD12 to PD0) in rodent . . . . . . . . . 27

1.7.3 The effect of first and second trimester PAE on cortical neurogenesis in rodent . . . . . . . . . . 28

1.8 THE EFFECT OF PAE ON THE SENSORIMOTOR SYSTEM . . 30

1.8.1 Behavioral alterations in children with FASD . . . . 30

1.8.2 CNS structural disruptions following PAE . . . . . 31

1.8.3 PAE sensorimotor disturbances in rodent . . . . $\quad . \quad 33$

1.9 TERATOGENIC EFFECT OF ALCOHOL ON HUMAN BRAIN AND
BEHAVIOR .
.

1.9.1 Factors contributing to the effect associated with FASD $\quad . \quad$. $\quad 37$

1.9.2 Mechanisms mitigating brain deficits following PAE . . 39

1.9.3 PAE and oxidative stress in the developing CNS . . . . 41 
1.9.3.1 Generation of ROS from aldehyde, the by-product of ethanol metabolism . . . . . . 42

1.9.3.2 Additional ethanol-induced ROS production and cell death . $\quad 43$

1.9.3.3 ROS produces cell death . . . . . . . 44

1.10 HUMAN VERSUS ANIMAL BLOOD ALCOHOL LEVEL · . 45

CHAPTER 2: THE LONG-TERM EFFECT OF PRENATAL ALCOHOL EXPOSURE ON POSTEROMEDIAL BARREL SUBFIELD IN JUVENILE AND ADULT RATS

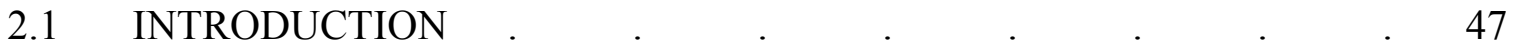

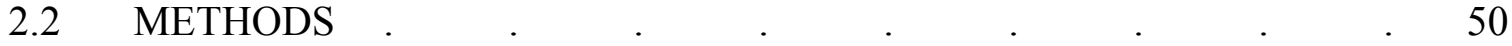

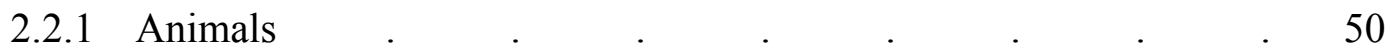

2.2.2 Breeding and treatment groups $\quad . \quad$. $\quad . \quad$. $\quad . \quad 50$

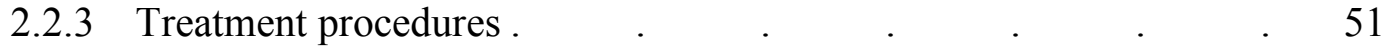

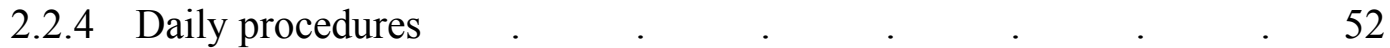

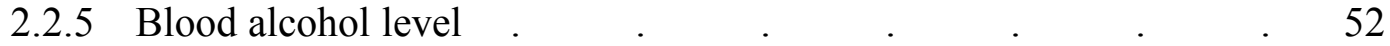

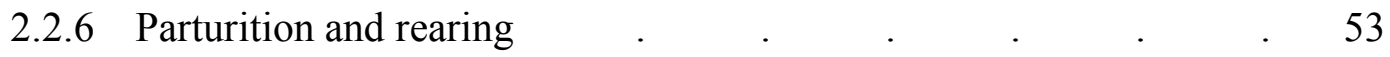

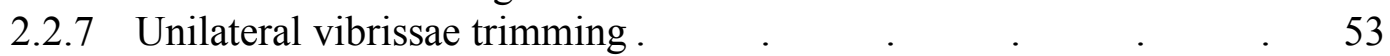

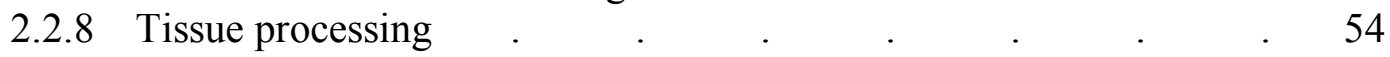

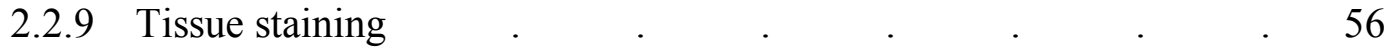

2.2.10 Quantitative PMBSF morphometric analysis . . . . $\quad$. 57

2.3 RESULTS . $. \quad . \quad . \quad . \quad . \quad . \quad . \quad .60$

2.3.1 Maternal blood alcohol levels. $\quad . \quad$. $\quad . \quad$. $\quad . \quad$. $\quad 60$

2.3.2 Effect of PAE on PMBSF in juveniles $\quad . \quad$. $\quad . \quad . \quad$. 61

2.3.2.1 The pattern of PMBSF in juveniles . . . . . . 61

2.3.2.2 The area of PMBSF in juveniles . . . . . . . $\quad$. 61

2.3.2.3 PMBSF barrel area of juveniles . . . . . . . 63

2.3.2.4 PMBSF septal area of juveniles . . . . . $\quad .63$

2.3.2.5 PAE produced intraregional asymmetries in PMBSF of juveniles . . . . . . . $\quad .63$

2.3.2.5.1 PMBSF barrel rows in juveniles . . . $\quad 65$

2.3.2.5.2 PMBSF barrel arcs in juveniles . . . $\quad 65$

$\begin{array}{lllllll}\text { 2.3.3 Effect of PAE on body weight in juveniles . } & \text {. } & \text {. } & 67\end{array}$

$\begin{array}{llllll}2.3 .4 & \text { Effect of PAE on brain weight in juveniles . } & \text {. } & 67\end{array}$

2.3.5 Forebrain/PMBSF ratio in juveniles . $\quad . \quad$. $\quad . \quad .69$

2.3.6 Correlation between forebrain weight and total PMBSF area in

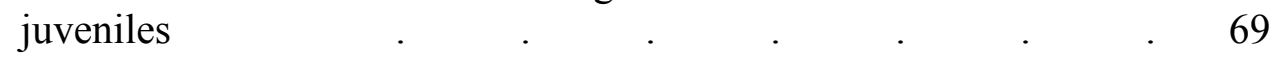

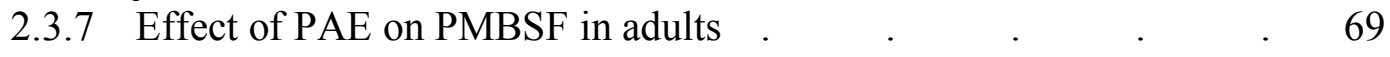

2.3.7.1 PMBSF barrel pattern in adults . . . . . $\quad .69$

2.3.7.2 The PMBSF area in adults . . . . . . . 71

2.3.7.3 The PMBSF barrel area in adults . . . . . . 71

2.3.7.4 PMBSF septal area in adults . . . . . . . 71

2.3.7.5 PMBSF intraregional vulnerability to PAE in adults . . 73

2.3.7.5.1 PMBSF barrel rows in adults . . . . 73

2.3.7.5.2 PMBSF barrel arcs in adults . . . . 76 
2.3.8 Effect of PAE on brain and body weights in adults . . . 76

2.4 THE EFFECT OF PAE AND VIBRISSAE TRIMMING ON PMBSF IN

JUVENILES . . . . . . . . . . . 76

2.4.1 Pattern of PMBSF in vibrissae trimmed juveniles . . . $\quad$. 78

2.4.2 The area of the PMBSF in trimmed EtOH and CF juveniles . $\quad 78$

2.4.3 PMBSF barrel area in trimmed EtOH and CF juveniles . $\quad$. 78

2.4.4 Septal area in trimmed EtOH and CF juveniles . $\quad . \quad$. $\quad 78$

2.4.5 Intraregional vulnerability in vibrissae trimmed $\mathrm{EtOH}$ and $\mathrm{CF}$

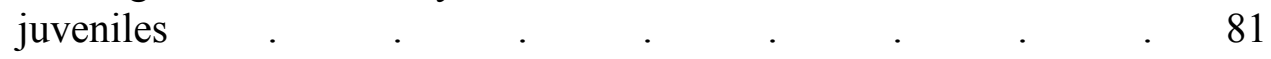

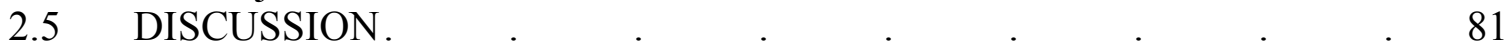

2.5.1 PAE has a long-term effect on the somatosensory cortex . $\quad$. 82

$\begin{array}{lllll}\text { 2.5.2 } & \text { PMBSF asymmetries in juveniles and adults . } & \text {. } & \text {. } & 84\end{array}$

2.5.2.1 Asymmetric variability within the CNS . $\quad . \quad$. 84

2.5.2.2 Asymmetries within the PMBSF following PAE . $\quad$. 85

2.5.2.3 Possible peripheral substrates influencing the anterior-to-posterior PMBSF asymmetry . . . $\quad$. 85

2.5.2.4 Possible CNS substrates influencing the anterior-to-posterior

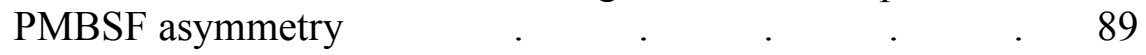

2.5.3 The effect of PAE and vibrissae trimming on the area of the PMBSF

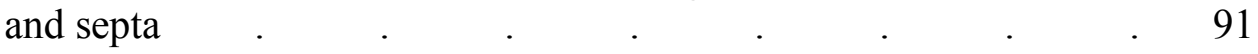

2.5.4 Body and brain weights of juveniles and adults $\quad . \quad$. $\quad . \quad 993$

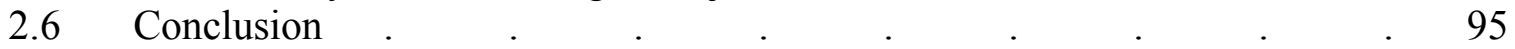

CHAPTER 3: PRENATAL ALCOHOL EXPOSURE REDUCED THE AREA OF THE GLABROUS FOREPAW AND FOREPAW BARREL

SUBFIELD .

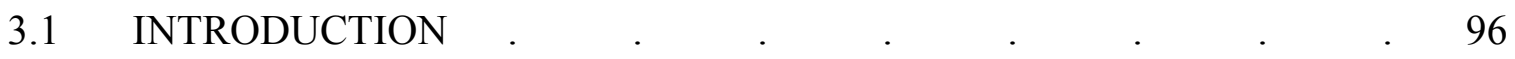

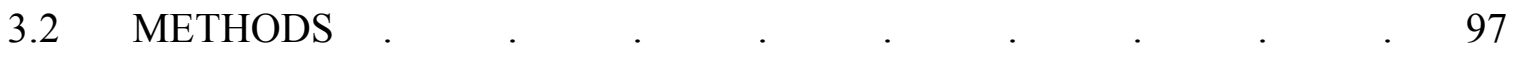

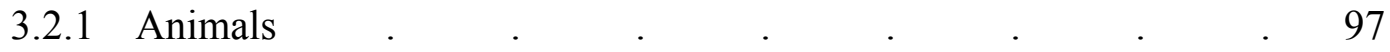

3.2.2 Breeding and treatment groups $\quad . \quad$. $\quad . \quad$. $\quad . \quad$. $\quad .98$

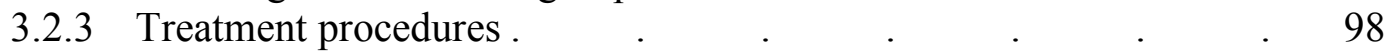

3.2.4 Quantitative FBS morphometric analysis . . . . . $\quad .98$

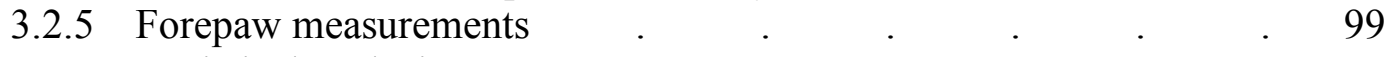

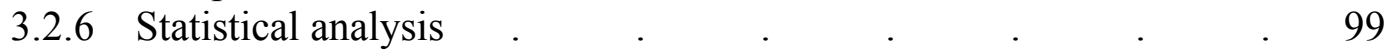

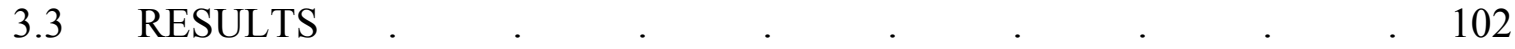

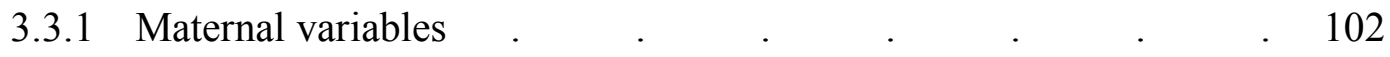

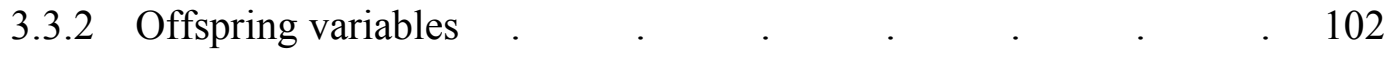

3.3.2.1 The effect of PAE on the area of the FBS . . . 102

3.3.2.2 The effect of PAE on the area of D2v to D5v barrels in FBS. 102

3.3.3 Effect of PAE on the area the of glabrous forepaw . . . . 104

3.3.3.1 Total glabrous forepaw area . . . . . . . . . 104

3.3.3.2 Glabrous digit area . . . . . . . . . . . 104

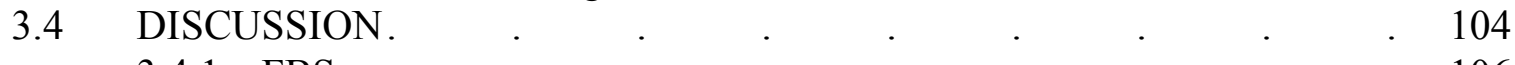

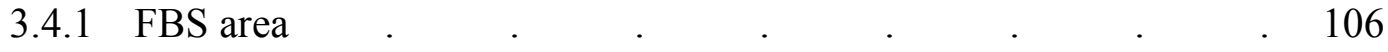

3.4.2 Glabrous forepaw and total glabrous digit area $\quad . \quad$. $\quad . \quad 108$ 
CHAPTER 4: PRENATAL ALCOHOL EXPOSURE REDUCED THE AREA OF THE FOREPAW REPRESENTATION IN SOMATOSENSORY CORTEX

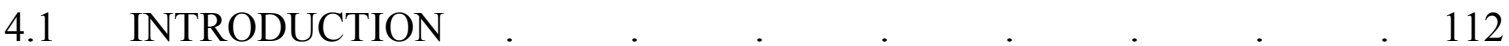

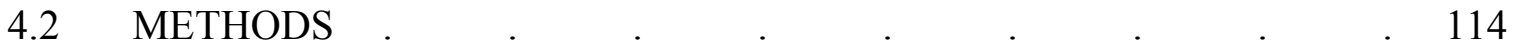

4.2.1 Animals, breeding, and treatment groups _ . . . . . . $\quad$. 114

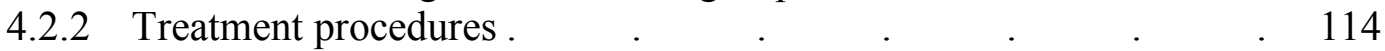

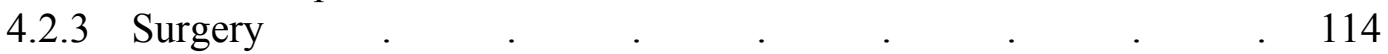

4.2.4 Physiological mapping and map reconstruction of primary receptive fields in the forepaw representation of SI . . . . . . 115

4.2.5 Evoked response latencies . . . . . . . . . . . . . 116

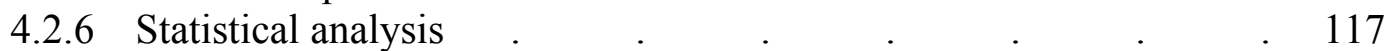

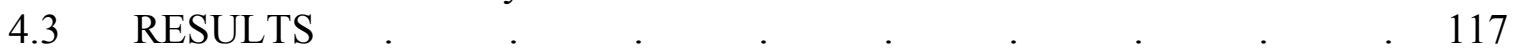

4.3.1 Maternal blood alcohol levels. $\quad . \quad$. $\quad . \quad$. $\quad . \quad$. 117

4.3.2 Effect of PAE on the forepaw representation in SI . . $\quad$. 117

4.3.2.1 Effect of PAE on the total ventral forepaw representation . 118

4.3.2.2 Total forepaw representation . . . . . . . . 118

4.3.2.3 D2vd to D5vd representation . $\quad . \quad$. $\quad$. $\quad$. 121

4.3.2.4 D2v to D5v representation . . . . . . . . $\quad$. 121

4.3.2.5 D2d to D5d representation . . . . . . . . . 121

4.3.2.6 Individual digit representations _ . . . . . . $\quad$. 122

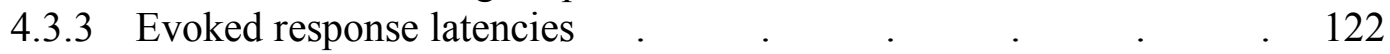

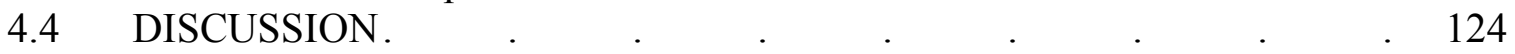

4.4.1 PAE reduced the forepaw representation in juvenile rats . $\quad$. 125

4.4.2 Increased evoked response latencies in EtOH and PF groups — 129

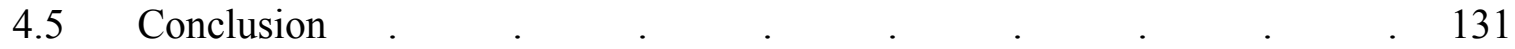

CHAPTER 5: THE EFFECT OF PRENATAL ALCOHOL EXPOSURE ON THE PRIMARY MOTOR CORTEX. . . . . . 132

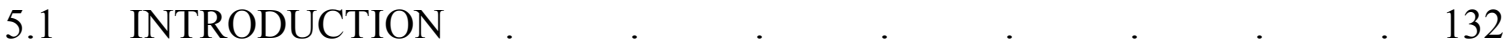

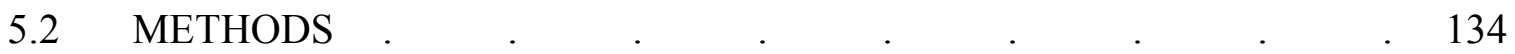

5.2.1 Breeding of dams and treatment groups $\quad . \quad$. $\quad . \quad . \quad . \quad$. 134

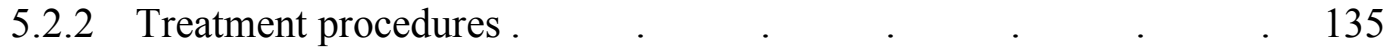

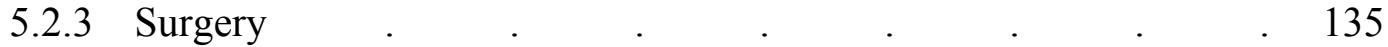

5.2.4 Microstimulation and motor mapping _ . $\quad . \quad$. $\quad . \quad$. 136

5.2.5 Motor cortex map reconstruction . . . . . . . . 138

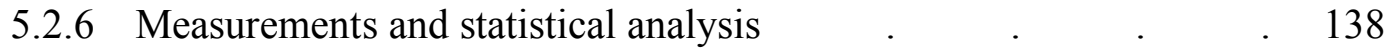

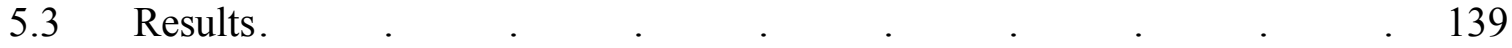

5.3.1 Maternal blood alcohol level . $\quad . \quad$. $\quad . \quad$. $\quad . \quad$. $\quad 139$

5.3.2 The effect of PAE on the area of forepaw and vibrissae
representations in MI .

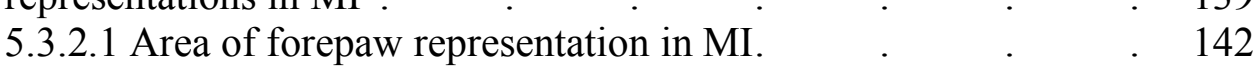

5.3.2.2 Area of the vibrissae representation in MI . . . . 142 
5.3.2.3 Combined areas of forepaw and vibrissae representations in $\mathrm{MI}$

5.3.3 The effect of PAE on ICMS thresholds for forepaw and vibrissae representation in $\mathrm{MI}$ 5.3.3.1 ICMS thresholds in forepaw MI

5.3.3.2 ICMS thresholds in vibrissae MI . . . . . 144

5.3.3.3 Total ICMS thresholds for vibrissae and forepaw $\begin{array}{lllllll}\text { representation in MI } & \text {. } & \text {. } & \text {. } & \text {. } & & 144\end{array}$

5.4 Discussion

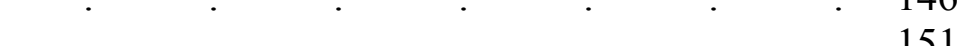

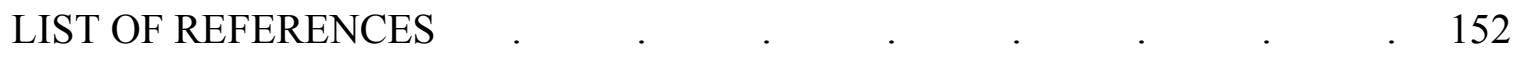

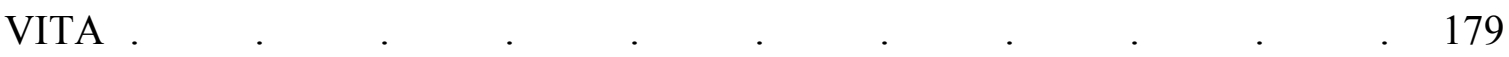




\section{LIST OF TABLES}

Table 2.1 PMBSF areas in juveniles $\quad . \quad$. $\quad . \quad$. $\quad . \quad$. $\quad 62$

Table 2.2 The effect of PAE on body, brain weights, and forebrain/

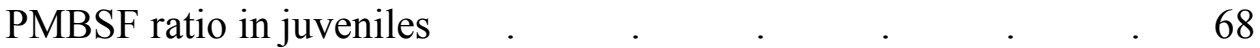

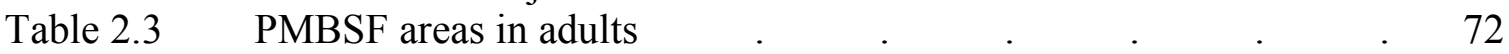

Table 2.4 The effect of PAE on body and brain weights in adults . . 77

Table 2.5 PMBSF areas in EtOH-trim, EtOH-intact, CF-intact, and CF-trim

groups .

Table 3.2 PAE reduced the area of the FBS $\quad . \quad \ldots \quad . \quad . \quad . \quad . \quad 103$

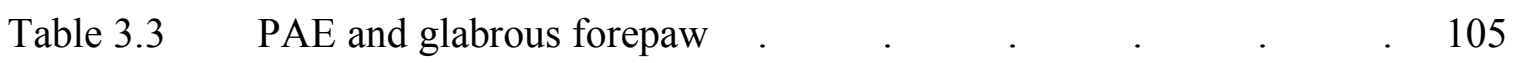

Table 4.1 Electrophysiological forepaw representations $\quad . \quad$. $\quad 120$

Table 4.2 Longer evoked response latencies occurred in the EtOH and PF

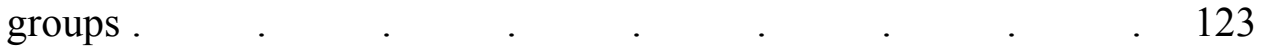

Table 5.1 Forepaw and vibrissae motor map areas with thresholds $\leq 100 \mu \mathrm{A} \quad . \quad 143$

Table $5.2 \quad$ Average threshold $(\leq 100 \mu \mathrm{A})$ needed to produce motor maps $\quad . \quad 145$ 


\section{LIST OF FIGURES}

Figure 1.1 Mystacial vibrissae, forepaw, and the barrel field _ . . . 11

Figure 1.2 Physiological representation of forepaw in SI $\quad . \quad$. $\quad$. 14

Figure 2.1 Trimmed unilateral mystacial pads in juvenile rats . $\quad . \quad 55$

Figure 2.2 The juvenile barrel cortex emphasizing PMBSF barrel pattern . 58

Figure 2.3 Barrel asymmetries within the PMBSF of PAE juvenile rats. $\quad 64$

Figure 2.4 Juvenile intraregional arc and row vulnerability within PMBSF $\quad$. 66

Figure 2.5 Correlation between forebrain weight and total PMBSF area in

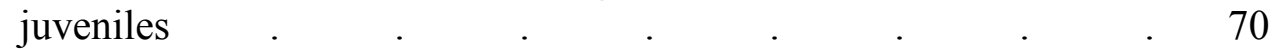

Figure 2.6 Barrel asymmetries within the PMBSF of PAE adults . $\quad$. 74

Figure 2.7 Intraregional PMBSF arc and row vulnerability within PMBSF of adults . . . . . . . . . 75

Figure 2.8 The PMBSF following unilateral vibrissae trimming in CF-trim, CF-intact, EtOH-trim, and EtOH-intact groups . . . . 79

Figure 3.1 Total FBS and ventral digit representation for each group . $\quad$. 100

Figure 3.2 Glabrous forepaw and digit areas _ . $\quad . \quad$. $\quad . \quad$. 101

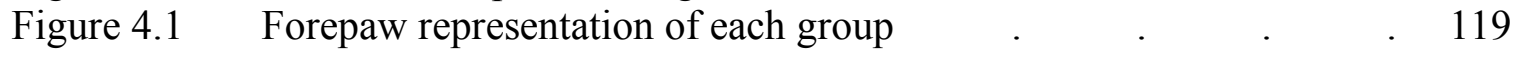

Figure 5.1 Forepaw and vibrissae motor map overlying cortical MI surface. $\quad 140$

Figure 5.2 MI maps of forelimb and vibrissae evoked motor responses

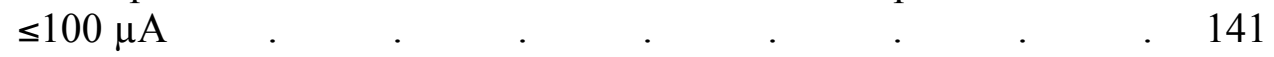




\section{LIST OF ABBREVIATIONS}

2DG

5-HT

ADHD

AGl

AGm

ALBSF

ANOVA

ARBD

ARND

BAL

BBB

$\mathrm{CF}$

CFL

$\mathrm{Cg} 1$

CNS

$\mathrm{CO}$

CYP2E1

D

d

EtOH

EtOH-trim

EtOH-intact

FAS

FASD

FBS

FP

FSC

GD

HBS

$\mathrm{Hd}$

HP

HRP

HT

ICMS

KPBS

LL

LLBSF

$\mathrm{M}$

MGE

MI

$\mathrm{NADH}$

NaPBS 2-deoxyglucose

serotonin

Attention deficit and hyperactivity disorder

Agranular lateral field

Agranular medial field

Anterolateral barrel subfield

Analysis of variance

Alcohol related-birth defect

Alcohol related-neurodevelopmental disorder

Blood alcohol level

Blood brain barrier

Chowfed

Caudal forelimb motor cortex

Cingulate area 1

Central nervous system

Cytochrome oxidase

Cytochrome P-4502E1

Digit

Dorsal

Ethanol

Ethanol+contralateral mystacial pad trimmed

Ethanol+ipsilateral mystacial pad trimmed

Fetal alcohol syndrome

Fetal alcohol spectrum disorder

Forepaw barrel subfield

Forepaw

Follicle sinus complex

Gestational day

Hindpaw barrel subfield

Hand dorsal

Hindpaw

Horse radish peroxidase

Hypothenar pad

Intracortical microstimulation

Potassium phosphate buffered saline

Lower-lip

Lower lip barrel subfield

Middle

Medial ganglionic eminence

Primary motor cortex

Nicotinamide adenine dinucleotide

Sodium phosphate buffered saline 


$\begin{array}{ll}\text { NR } & \text { No response } \\ \text { OS } & \text { Oxidative stress } \\ \text { P } & \text { Pad } \\ \text { PAE } & \text { Prenatal alcohol exposure } \\ \text { PD } & \text { Postnatal day } \\ \text { PF } & \text { Pairfed } \\ \text { PL } & \text { Posterior lateral vibrissae motor cortex } \\ \text { PMBSF } & \text { Posteromedial barrel subfield } \\ \text { POm } & \text { Medial division of posterior thalamic nucleus } \\ \text { RFL } & \text { Rostral forelimb motor cortex } \\ \text { ROS } & \text { Reactive oxygen species } \\ \text { SES } & \text { Socioeconomic status } \\ \text { SI } & \text { Primary somatosensory cortex } \\ \text { SII } & \text { Second somatosensory cortex } \\ \text { TH } & \text { Thenar pad } \\ \text { Tks } & \text { Trunk barrel subfield } \\ \text { V } & \text { Ventral } \\ \text { VB } & \text { Ventrobasal thalamus } \\ \text { Vib } & \text { Mystacial vibrissae } \\ \text { VPL } & \text { Ventral posterior lateral thalamic nucleus } \\ \text { VPM } & \text { Ventral posterior medial thalamic nucleus } \\ \text { XF } & \text { Cross foster }\end{array}$




\section{CHAPTER 1 \\ INTRODUCTION}

\subsection{FETAL ALCOHOL SYNDROME}

Consumption of alcohol by a pregnant mother that results in a high blood alcohol level (BAL) is frequently associated with life-long damage to a child, culminating in: 1) facial dysmorphologies, 2) growth retardation, and 3) central nervous system (CNS) disruptions (Jones, et al., 1973). When all three deficits occur in humans, the patient is diagnosed as having "fetal alcohol syndrome" (FAS). Some investigators have reported the FAS incidence rate at 0.33 to 1.9 per 1000 live births (Abel \& Sokol, 1987; Abel \& Sokol, 1991; May \& Gossage, 2001) while others reported incidence rates as high as $>40$ cases per 1,000 births (May, et al., 2000) [these differences reflect variations in the population under investigation]. While FAS typically only occurs in women who consume high levels (as defined in section 1.9.1) of alcohol (typically in a binge-type and chronic manner) during pregnancy, it is interesting to note that FAS is the most preventable cause of mental retardation in the western world and would completely cease if women refrained from alcohol consumption during pregnancy. The observable history of alcohol's adverse side-effects on children originally dates to ancient times as described by Aristotle (Abel, 1999). In the 1700s, groups of physicians described the feeble and weak nature of children born to alcoholics (Hoyme, et al., 2005). By 1899 alcohol abuse was beginning to be associated with high miscarriage rates and varying levels of birth defects (Hoyme, et al., 2005). In 1968 Lemoine examined children of alcoholic mothers and documented several physical and behavioral patterns similar to those later reported as 
FAS (Chaudhuri, 2000; Hoyme, et al., 2005). However, it was not until 1973 when Jones and Smith first coined the term FAS with its classic symptoms defining this syndrome (Jones \& Smith, 1973; Jones, et al., 1973).

\subsection{FETAL ALCOHOL SPECTRUM DISORDER}

FAS is useful in describing the most severe examples of the teratogenic effects of chronic, binge-type, gestational alcohol exposure, hereafter referred to as "prenatal alcohol exposure" (PAE) [this term is used here for humans and rodents]. However, a more general term was needed to represent the broad range of structural, behavioral, and neurocognitive disorders frequently associated with PAE. To this end, fetal alcohol spectrum disorder (FASD) was implemented as an umbrella term covering the full range of PAE-related effects. FASD is used to cover: alcohol-related neurodevelopmental disorder (ARND) (Sampson, et al., 1997), alcohol-related birth defects (ARBD) (Coles, et al., 1991), fetal alcohol abuse effects or partial-FAS, fetal alcohol effects (Rosett, Weiner \& Edelin, 1981; Warren \& Foudin, 2001) and FAS. Therefore, while FASD is not a diagnostic term, it is an all-encompassing descriptive term that covers the breadth and depth of the multiple teratogenic effects (ARND, ARBD, FAS, partial-FAS) often seen in children following PAE. Due to the expansiveness of FASD as well as to facilitate understanding by covering the numerous terms associated with PAE, the term FASD will be primarily used throughout this dissertation when reviewing human effects associated with abusive gestational alcohol exposure. (Goodlett, Horn \& Zhou, 2005; Hoyme, et al., 2005; Riley \& McGee, 2005; Warren \& Li, 2005). 
In 1996, the Institute of Medicine categorized the criteria used to assist in diagnosing children that fell under the FASD umbrella (Stratton, Howe \& Battaglia, 1996). However, as investigators continue to understand the more detailed aspects of PAE, the original criteria have been updated and refined and can be found in current literature reviews (Hoyme, et al., 2005).

Often, children with FASD show severe behavioral impairments associated with sensorimotor processing deficits as evidence by their poor performance on higher-order cognitive motor tasks (Adnams, et al., 2001), difficulties in maintaining postural balance (Roebuck, et al., 1998b), slower premotor and motor reaction times (Simmons, et al., 2002), and deficits in fine motor control (Connor, et al., 2006), and coordinated movement timing (Wass, et al., 2002). Many of these deficits in sensorimotor processing may be due to the teratogenic effect of PAE on the parietal cortex (Archibald, et al., 2001) which is the major center of somatosensory information processing.

\subsection{THE PRIMARY SOMATOSENSORY CORTEX}

The somatosensory system provides a systematic representation of the peripheral world impinging upon the body surface. Specialized receptors in the skin transduce mechanical, thermal, or nociceptive sensations and relay this information to the nervous system (Kandel \& Schwartz, 1985). The somatosensory system is responsible for processing three types of somatic sensations: 1) Exteroceptive sensations from mechanoreceptors (touch), thermoreceptors (temperature), and nociceptors (pain) provide information from the outside world; 2) Proprioceptive sensations from joints, ligaments, and tendons provide information about body position, current state of muscle contraction, 
and tendon tension; and 3) Interoceptive sensations from receptors in the viscera provide information on the state of internal organs (Larry, et al., 2003).

\subsubsection{Circuitry pathways: periphery to cortex}

Peripheral sensory information is relayed to spinal cord, brainstem, cerebrum, and cerebellum. Tactile input for touch is primarily relayed ipsilaterally to medullary dorsal column nuclei where fibers from upper body (or forelimb/rostral half of body in rats) project to the cuneate nucleus while lower body (hindlimb region in rats) inputs project to the gracilis nucleus (Kandel \& Schwartz, 1985). Sensory inputs for pain, temperature, crude touch, and limb proprioception are relayed into the dorsal horn of the spinal cord. Tactile input from the head and neck is relayed to trigeminal nucleus principalis, while pain and temperature inputs are relayed to the spinal trigeminal nuclei (Williams, Zahm \& Jacquin, 1994).

Medullary cells in the dorsal column nuclei send their axons across the midline where they enter the medial lemniscal fiber tract and travel to the ventral basal complex (VB) in the thalamus. Tactile input from the body below the neck terminates in the ventral posterior lateral nucleus (VPL) of the thalamus whereas input from the head and neck terminate in the ventral posterior medial nucleus (VPM) of the thalamus. In the spinal cord, axons of pain and temperature sensory cells in the dorsal horn ascend within one or two spinal segments before crossing the spinal cord to become part of the spinothalamic tract within the anterolateral system. The spinothalamic tract axons merge with medial lemniscal fibers and terminate in VPL and posterior medial thalamus (POM) (Kandel \& Schwartz, 1985; Larry, et al., 2003). Medial lemniscal fibers are separated into 
1) lemniscal fibers, relaying in VB and 2) paralemniscal fibers, relaying in POM (Diamond, 1995; Diamond, et al., 1992).

VB axons enter the internal capsule and terminate, in large part, in primary somatosensory cortex (SI) layer IV with a few axons making synapses on layer III cells (Arnold, Li \& Waters, 2001; Jensen \& Killackey, 1987); secondary somatosensory cortex (SII), and motor cortex (Kandel \& Schwartz, 1985) also receive some afferent projections. POM neurons send their axons to layers II through VI in SI. Spiny stellate neurons in layer IV receive thalamic terminals (Lubke, et al., 2000). Layer IV neurons in turn project to supragranular layer II/III (Erzurumlu \& Jhaveri, 1990; Kim \& Ebner, 1999; Lubke, et al., 2003). Layer II/III neurons project to infragranular layer V and layer VI, largely within a radially oriented column. Infragranular layer neurons project to motor cortex, SII, cerebellum and basal ganglia. Cells in both supra and infragranular layers project through the corpus callosum to the contralateral hemisphere (White, 1989).

\subsubsection{From cortical columns to cortical somatotopy}

Lorente de Nó first described a columnar organization for somatosensory cortex based on anatomical evidence indicating that neurons traversed the cortex in organized, vertical pathways (Lorente de No, 1922). Mountcastle later noted that all cells encountered in a given vertical electrode penetration responded to input from similar regions of the body surface. This highly organized feature extending radially through the cortex was described as a cortical "column" since cells throughout the depths responded to input from a restricted region of the body surface (Mountcastle, 1957; Mountcastle,

1958; Mountcastle, Davies \& Berman, 1957). Columns were initially thought to be 300 to 
$400 \mu \mathrm{m}$ wide, but were subsequently divided into smaller units termed "minicolumns" that were 50 to $60 \mu \mathrm{m}$ in diameter (Mountcastle, 1997a). The minicolumn was defined by Mountcastle as the basic modular unit of the neocortex (Mountcastle, 1978). Rakic later described how neurons migrated along radial glia processes during corticogenesis (Rakic, 2003) to produce ontogenic columns. The ontogenic columns were hypothesized to be the basic processing units in the adult cortex and were closely related to Mountcastle minicolumns (Horton \& Adams, 2005; Rakic, 1988). Although columns have been well described (Diamond, et al., 2003; Favorov \& Diamond, 1990; Panzeri, et al., 2003; Vierck, Favorov \& Whitsel, 1988), others still question the purpose, function, and role (if any), of columns in contributing to perception (Horton \& Adams, 2005; Rockland \& Ichinohe, 2004).

Anatomical studies of somatosensory cortex (Bernardo, et al., 1990; Kim \& Ebner, 1999) have shown that cell bodies in cortical layer IV barrels have dendrites that stay mostly confined to cortical regions directly above the cell body of the dendrite in supragranular layers II/III, while axons stay confined to a pattern within their layer IV barrel (Lubke, et al., 2000; Petersen \& Sakmann, 2000). Anterograde tracers injected into thalamus revealed that individual axonal projections to cortex are largely segregated into specific regions that stay mostly confined to cell-scarce interbarrel cortical septa (receives fewer connections) or cell-dense barrel cortex [receives the majority of projections] (Lu \& Lin, 1993). The anterograde tracers Phaseolus vulgaris leucoagglutinin and horseradish peroxidase, injected into the posterior medial thalamus, labeled neuronal projections that were distributed throughout the septal region in upper layer $\mathrm{V}$ to layer I. When these tracers were injected into ventral posterior medial 
thalamus (VPM), neuronal projections were mostly confined to cortical regions (layer VI to layer I) directly above and below layer IV barrels. The majority of the labeled fiber terminals were identified within the barrel region of layer IV (Koralek, Jensen \& Killackey, 1988; Lu \& Lin, 1993). Columns with similar cortical synaptic patterns have also been described in visual (Hubel, Wiesel \& Stryker, 1978; LeVay, Hubel \& Wiesel, 1975; LeVay, Wiesel \& Hubel, 1980) and auditory (Imig \& Brugge, 1978; Wallace \& Bajwa, 1991; Winer, 1984) cortices. The general notion of columnar organization is supported by physiological studies in auditory (Imig \& Adrian, 1977; Scheich, 1983), visual (Crawford, Meharg \& Johnston, 1982; Kanold, et al., 2003; Singer, 1981; WongRiley, 1979) somatosensory (Simons, 1978; Welker, et al., 1993) and motor cortices (Asanuma \& Rosen, 1972b; Mountcastle, 1997b; Rosen \& Asanuma, 1972). Using 2deoxyglucose, metabolically active regions were found to extend throughout cortical layers in a columnar pattern supporting the idea that cortical activity is generally confined to a specific section of cortex from layer VI to layer II/III (Durham \& Woolsey, 1977; Kossut \& Juliano, 1999; McCasland, et al., 1991; McCasland \& Woolsey, 1988). Additional studies examining cortical columns in primates (Kaas, et al., 1979; Lin, et al., 1979; Nelson, et al., 1980; Sur, Nelson \& Kaas, 1980; Welker, et al., 1957) and rodents (Waters, McCandlish \& Li, 1995; Welker \& Woolsey, 1974; Woolsey \& Van der Loos, 1970; Woolsey, Welker \& Schwartz, 1975) have led to somatosensory maps and the understanding that peripheral receptors form a topographic map in SI. The underlying assumption is that cortical columns receive input from a restricted region of the body surface. By systematically moving electrodes across cortex it became clear that the columnar arrangement was part of a large topographically oriented map of the body 
surface. During neurosurgery, Penfield (Penfield, 1972; Penfield \& Boldrey, 1937) used physiological methods to map the representation of the human body surface. Detailed maps of the body surface in animals were then made by Woolsey in monkey (Woolsey, Marshall \& Bard, 1942) and cat (Woolsey, 1964). Later his work, dealing with how sensory information is represented in the cortex, was continued by many of his students (Kaas, et al., 1979; Merzenich, et al., 1983; Van der Loos \& Woolsey, 1973; Woolsey, Welker \& Schwartz, 1975). The highly organized somatotopy of the cortex is not restricted to SI alone, as thalamus (Manger, Rosa \& Collins, 2001) and brainstem (Jacquin, Rhoades \& Klein, 1995) are also organized into somatotopic representations as well. The use of rats and mice has greatly facilitated an understanding of cortical somatotopy due to their highly organized layer IV barrel cortex.

\subsection{THE RODENT BARREL CORTEX}

\subsubsection{Barrelettes, barreloids, and barrels}

The somatosensory system, in rodent as in all other mammals, is somatotopically arranged with the representation of the body surface found at the DCN, VB, SI, and SII. However, unlike other mammals, a cell organization exists at each relay station that represents the body surface. These cell arrangements can be visualized with any number of stains, including Nissl (cell), cytochrome oxidase (mitochondria), or serotonin antibody. The manner in which cells aggregate into dense clusters and form the body representation has been termed "barrels" in the cortex (Killackey, 1973; Woolsey, Dierker \& Wann, 1975; Woolsey, Welker \& Schwartz, 1975), "barreloids" in thalamus 
(Haidarliu \& Ahissar, 2001; Land, Buffer \& Yaskosky, 1995; Senft \& Woolsey, 1991; Welt \& Steindler, 1977; Zantua, et al., 1996) and "barrelettes" in dorsal column nuclei (Ma, 1993). Due to the high degree of somatotopic organization at each ascending synaptic site and the general one-to-one topography between periphery and barrelette, barreloid, or barrel, the rodent somatosensory system is a valuable model system for studying somatosensory information processing (Margret, et al., 2006a; Margret, et al., 2006b; McCandlish, Li \& Waters, 1993; Schlaggar \& O'Leary, 1993; Schlaggar \& O'Leary, 1994; Waters, McCandlish \& Li, 1995), learning (Ebner \& Armstrong-James, 1990; Mierau, et al., 2004; Rema, Armstrong-James \& Ebner, 2003), plasticity, cortical reorganization (Pearson, et al., 2001; Pearson, et al., 2003), cortical connectivity (Petersen, et al., 2004; Staiger, et al., 1999) and sensory deprivation (Machin, et al., 2004; Maier, et al., 2003).

\subsubsection{Morphological organization of layer IV barrel cortex}

As first identified in 1922 by Lorente de Nó (Lorente de No, 1922), the barrel cortex is organized into punctate cell-dense aggregates forming structurally distinct cytoarchitectonic units in layer IV of SI called "barrels" (Woolsey \& Van der Loos, 1970). Each barrel represents a precise region of the skin surface. Larger groups of barrels form subfields associated with the representation of vibrissae, lips, and limbs

(Welker \& Woolsey, 1974; Woolsey, Welker \& Schwartz, 1975). Barrels are separated from one another by interbarrel regions called septa, that receive input from POM (Chmielowska, Carvell \& Simons, 1989). Because cytochrome oxidase is a mitochondrial stain, its increased staining in barrels indicates that cells in barrels have more 
mitochondria (and/or there are more cells) and are thus more metabolically active than septa (Wong-Riley \& Welt, 1980). Larger barrel subfields are typically associated with peripheral regions that are more active or require more cortical processing. Therefore, while the cortical representation of the vibrissae encompasses a rather large cortical area, the hindpaw representation is relatively quite small. The vibrissae and hindpaw subfields are but two examples of six total major subfields contained within the barrel cortex.

\subsubsection{PMBSF, ALBSF, and LLBSF}

The posterior medial barrel subfield (PMBSF), named because of its location, is associated with the representation of the mystacial vibrissae. The PMBSF, shown in Fig 1.1 A, is organized into five rostrocaudally running rows and six main medial-lateral running arcs correspond to the mystacial pad vibrissae (Woolsey \& Van der Loos, 1970). PMBSF rows contain 4 to 12 barrels, each associated with the representation of a single whisker on the contralateral face. The anterolateral barrel subfield (ALBSF) extends rostrally from the PMBSF and is associated with the representation of the sinus hairs on the face and snout. PMBSF and ALBSF can be separated by the medial notch lying between the edges of the medial portion of each subfield. The lower lip barrel subfield (LLBSF) is located directly medial to the ALBSF and is associated with the representation of the lower lip and chin.

\subsubsection{FBS}

The forepaw barrel subfield (FBS) is located medial to the PMBSF and posteromedial to the LLBSF (see Fig 1.1A). The FBS is associated with the 
Figure 1.1 Mystacial vibrissae, forepaw, and the barrel field.

Illustrated examples of the PMBSF, mystacial vibrissae, FBS, and peripheral forepaw in six-week-old rats. A) Photomicrograph of SI layer IV barrel field. Individual barrel subfields are noted. Due to the importance of the PMBSF and FBS in the current dissertation, these two subfields have been circumscribed and marked with additional information related to their specific barrel constitutions. Notice the similar row and arc patterns between the PMBSF barrel pattern and mystacial pad vibrissae. Each PMBSF barrel represents an individual mystacial vibrissa. The posterior PMBSF also contains four Straddler barrels $(\alpha, \beta, \gamma$, and $\delta)$. Note the medial notch separating the entire PMBSF from the anterolateral barrel subfield (ALBSF). Also note the dashed line indicating the last arc of PMBSF barrels used for data in the following chapters. The ALBSF is primarily representative of smaller vibrissae (not fur) around the nares as well as the buccal pad or the vibrissae lining the inner lip (not shown). Medial to the ALBSF is an oblong barrel subfield primarily associated with the lower lip (LLBSF). The FBS is located medial and posterior to the LLBSF. Continuing medial and posterior from the FBS is the hindpaw barrel subfield (HBS) and a triangular-shaped region devoid of barrels containing the representation of the trunk, shoulder and wrist, called the trunk subfield (Tks). B) Whisker pad in a six-week-old rat showing the five rows (A to E) of mystacial vibrissae. The location of the 27 main mystacial vibrissae has been marked with dots at the whisker base. All mystacial vibrissae rows have also been traced to elucidate their organized positions in relation to the mystacial pad. Vibrissae A1 (extreme dorsal and posterior on face), B2 (diagonal from A1 in a anteroventral direction), C3 (middle vibrissae of mystacial pad, located diagonal in an anteroventral direction from B2), D4 and E5 are shown below C3. Straddler vibrissae $\alpha, \beta, \gamma$, and $\delta$ are indicated along the posterior edge of the mystacial pad. The image of the ventral/glabrous region of the forepaw is shown in $\mathbf{C}$ ) The spatial placement of digit and pad representations in the FBS is similar to that in the FBS, shown in A. 

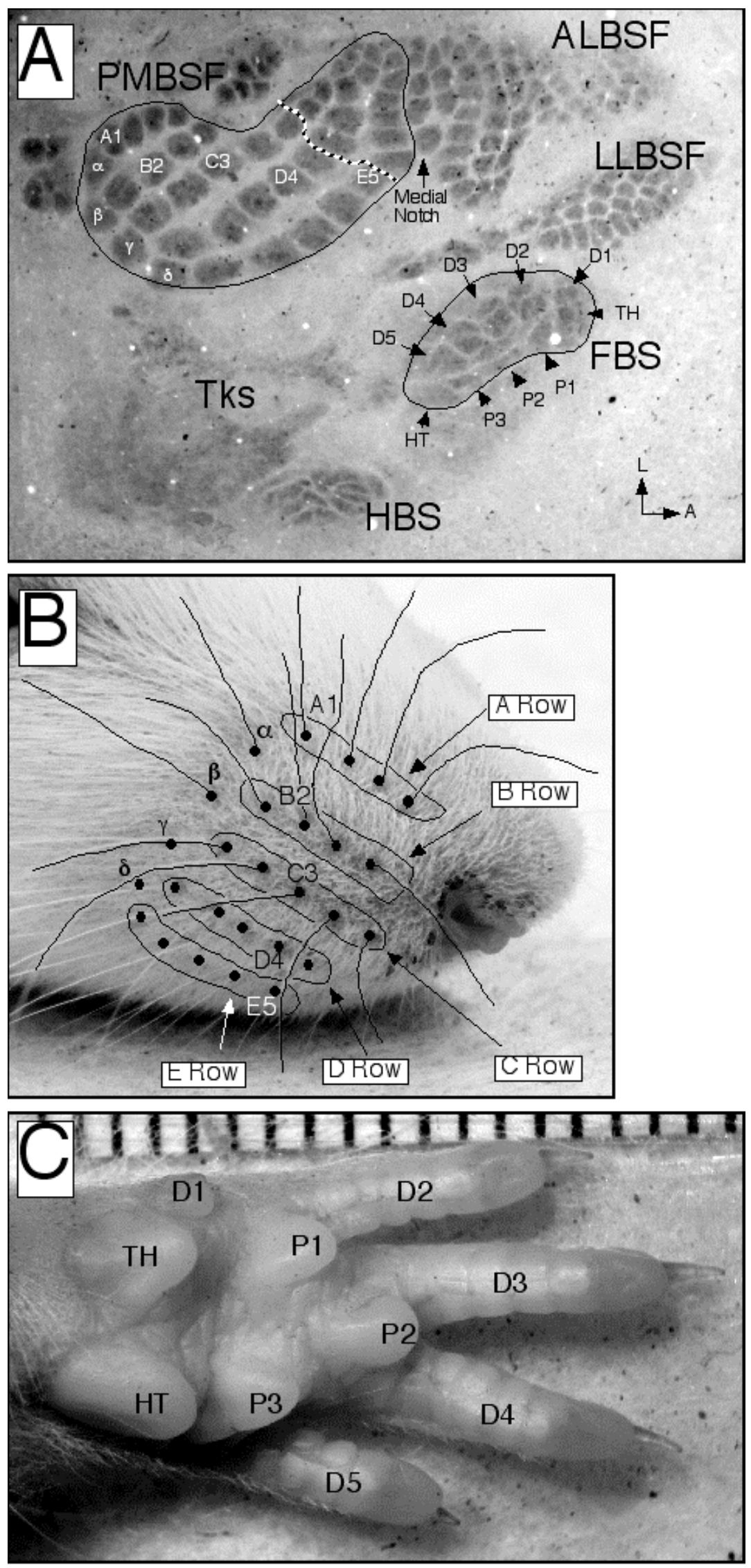
representation of the contralateral dorsal and ventral forepaw as shown in Fig 1.1C. The FBS contains four medial to lateral running bands; each band contains four to five barrels and represents the ventral surfaces of either digit 2 (D2), D3, D4, or D5. The anterior lateral portion of FBS consists of D1 and medial to D1 is the thenar pad (TH). The posteromedial region of FBS represents the hypothenar pad (HT). Medial to the ventral digit representation is the location of representative pads 1 (P1), P2, and P3, whereas dorsal digits 2 (D2d), D3d, D4d, and D5d are lateral to the digits is the dorsal hand (Hd). FBS and ventral forepaw regions are illustrated in Fig 1.1A and Fig 1.1C respectively.

\subsubsection{HBS and Tks}

The hindpaw barrel subfield (HBS) is located more medial and posterior to the FBS (Fig 1.1A). The HBS is associated with the representation of the hindpaw (Pearson, et al., 1996). The trunk subfield (Tks) represents trunk, chest, abdomen, back and shoulder representations as indicated in Fig 1.1A. Tks is not composed of barrels.

\subsubsection{SI physiological forepaw representation}

The morphological forepaw representation of FBS corresponds closely to a functional forepaw representation identified with electrophysiology in SI (Waters, Li \& McCandlish, 1995). The forepaw has been mapped in SI by several investigators (Chapin \& Lin, 1984; Waters, Li \& McCandlish, 1995; Welker, 1976). In these studies, microelectrodes were used to locate the receptive field of neurons in SI and generate a map of the forepaw representation. The forepaw representation in relation to surface vasculature and its reconstructed map are shown in Fig 1.2A and Fig 1.2B respectively. 


\section{Figure 1.2 Physiological representation of forepaw in SI.}

Fig 1.2 shows the details of the representation of forepaw in SI and the ventral surface of the forepaw. A) Surface view of right cortex showing the approximate location of the forepaw representation lying between the anterior and middle cerebral arteries and veins $($ scale $=1 \mathrm{~mm})$. Black box indicates the location of the forepaw representation. Asterisk denotes bregma at midline. B) Map of the forepaw representation from layer IV that was projected onto the cortical surface. Dots indicate individual electrode penetrations. Individual digit and pad sites of the forepaw are demarcated. A white line separates ventral and dorsal digit representations. Scale bar $=250 \mu \mathrm{m}$. C) Photomicrograph indicating the ventral forepaw and the similar spatial orientation of forepaw and forepaw representations. D) Photomicrograph of the dorsal hand. Scale bar segments each equal $1 \mathrm{~mm}$. 


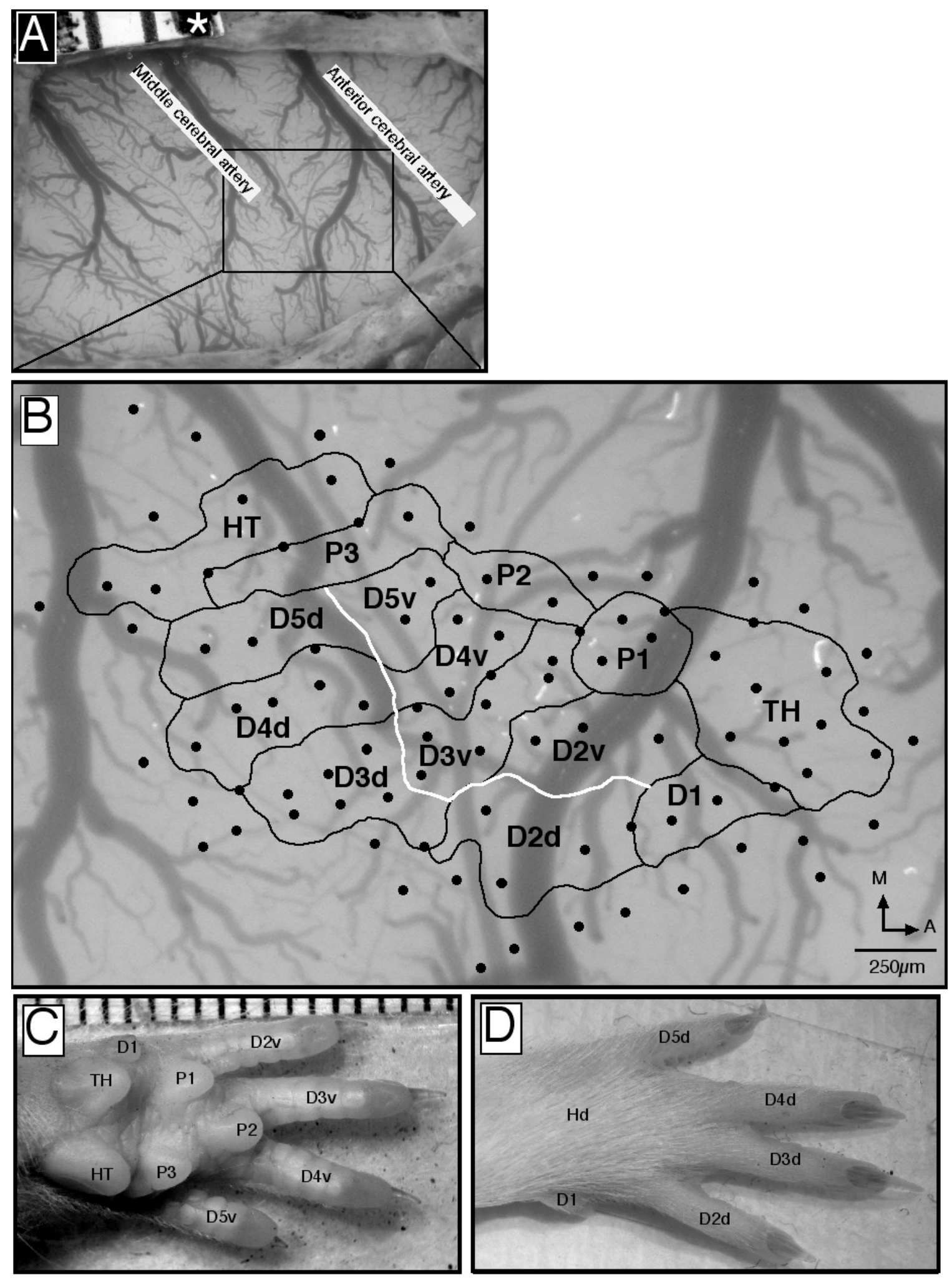


Forepaw representation maps are produced by placing extracellular electrodes into specific SI regions of layer IV cortex and recording single or multi-unit neuronal receptive fields after mechanically stimulating the periphery. In this dissertation the map was generated by lightly tapping the ventral and dorsal forepaw with a small, blunt, wire. The forepaw representation lies between the anterior and middle cerebral artery and vein as shown in Fig 1.2A. The reconstructed forepaw map shown in Fig 1.2B shows the general organization and location of a forepaw physiological map. Digit 1 (D1) is located at the anterolateral edge of most forepaw maps. Immediately medial to D1 is the thenar (TH) representation. Posterior to D1 and following a slightly posterior and medial direction are digit representations for D2, D3, D4, and D5. The most posteromedial receptive field to D5 is the hypothenar (HT) pad representation. Anterior to the hypothenar pad is palmar pad 3 (P3) followed by the anterolateral representation of palmar pad 2 (P2) and palmar pad 1 (P1). Medial to P1 to P3 are non-responsive zones or zones identified as "joint" owing to the diffuseness of their responses, unspecific with respect to exact regions of the forepaw. Rostral to TH and D1 is lower lip or neck responsive zones while posterior to HT is responsive to wrist stimulation (not shown). Finally, lateral to digit representations D1 to D5 is dorsal hand (Hd) followed by a nonresponsive zone (neither one shown). Cortical regions responsive to vibrissae stimulation are found lateral to the forepaw representation. Inspection of the forepaw indicates the presence of four easily identifiable digits D2 to D5 with a stunted D1 (Fig 1.2C). Three digit pads, P1 to P3, and two large palmar pads (thenar and hypothenar) are also observable. Dorsal hairy digits are not shown. Previously identified similarities between morphological and physiological representations of respective peripheral sensory areas 
(Waters, Li \& McCandlish, 1995) provide an excellent model to view the influence of perturbations to the periphery by examining barrel morphology and functional physiology.

\subsection{THE PRIMARY MOTOR CORTEX}

\subsubsection{History of motor cortex studies}

Once SI has processed information from the periphery, this information is then relayed to the primary motor cortex, which is responsible for eliciting fine motor movement. Hughlings Jackson hypothesized that motor functions were localized to specific regions of the brain responsible for the initiation of movement (Kandel \& Schwartz, 1985). This idea followed from his observations indicating that small cortical lesions were often associated with focal seizures. These seizures began in lesioned motor cortical regions responsible for the distal extremities and spread from distal extremities to more proximal areas of the body. Jackson correctly surmised that the seizure location was dependent upon the spread of seizure-related synchronized discharges across the cortex beginning in regions responsible for distal motor movement and passing to motor cortex responsible for proximal muscle contraction (Kandel \& Schwartz, 1985). Neurologist Gustaz Fritsch and Eduard Hitzig were able to confirm specific cortical regions in dogs that were responsible for different contralateral muscle contraction, using cortical surface stimulation. This provided the first direct evidence that distinct cortical areas were responsible for contralateral body regions (Brecht, et al., 2004a). David Ferrier, who demonstrated limb and eye movements in a monkey following stimulation of pre- and 
post-central gyri of the posterior parietal cortex, confirmed the Fritsch/Hitzig observations. Charles Sherrington discovered that the precentral gyrus in cat was the cortical zone that most readily evoked motor movement, thus solidifying the term "motor cortex". However, these early experiments had relied upon surface stimulation using high stimulus currents to evoke movements from major muscle groups. It was not until $\sim 1970$ that Hiroshi Asanuma and associates observed contractions of single muscles in response to intracortical microstimulation (ICMS) of the cortex, using very low stimulus currents (Asanuma \& Rosen, 1972a). ICMS has further been used to explore the organization of primary motor cortex (MI) in rodent (Brecht, et al., 2004a; Brecht, et al., 2004b; Donoghue \& Wise, 1982; Gioanni \& Lamarche, 1985; Hall \& Lindholm, 1974; KartjeTillotson, Neafsey \& Castro, 1985; Li \& Waters, 1991; Miyashita, Keller \& Asanuma, 1994; Neafsey, et al., 1986; Neafsey \& Sievert, 1982; Sievert \& Neafsey, 1986).

\subsubsection{Cortical cytoarchitecture related to motor maps: AGl, AGm, and Cg1}

Investigators that examined MI arrived at a general consensus regarding the cytoarchitectonic areas and subsequent borders to rat motor cortex, (Brecht, et al., 2004a; Donoghue \& Wise, 1982; Neafsey, et al., 1986) as explained below. Furthermore, the most recent study of rat motor cortex included extra- and intracellular stimulation (Brecht, et al., 2004a) techniques to further clarify any previous inconsistencies regarding MI's cytoarchitecture.

The motor cortex is divided into three major cytoarchitectonic areas: the agranular medial field (AGm), the agranular lateral field (AGl) and the cingulate area $1(\mathrm{Cg} 1)$, with each field evoking movement in a different body region following ICMS. Lateral and 
caudal to these regions are the granular and dysgranular fields, which consist of the barrels and septal regions of layer IV, and primarily represent the somatosensory area (Brecht, et al., 2004a).

\subsubsection{ICMS evoked motor maps}

Studies using microelectrodes, inserted perpendicular into rodent cortex, have been used to produce ICMS motor maps. The clearest body movements were produced when ICMS occurred at depths between $1500 \mu \mathrm{m}$ to $1700 \mu \mathrm{m}$ from the cortical surface, which typically represent layer V and upper layer VI when stimulated [deeper to 1700 $\mu \mathrm{m}$ runs the risk of stimulating deep white fiber tracts of the internal capsule] (Brecht, et al., 2004a). At this depth range, ICMS elicits movements with thresholds ranging from 10 to $100 \mu \mathrm{A}$, with a mean of $\sim 30 \mu \mathrm{A}$ (Brecht, et al., 2004a; Donoghue \& Wise, 1982;

Gioanni \& Lamarche, 1985; Neafsey, et al., 1986; Sanes, Suner \& Donoghue, 1990), but responses have also been reported at thresholds lower than $10 \mu \mathrm{A}$ in $\mathrm{AGm}, \mathrm{AGl}$ and $\mathrm{Cg} 1$.

\subsubsection{Eyelid/periocular movements respond to ICMS in Cg1}

Similar to the somatotopic organization of SI, MI is organized into a map of the musculature [musculotopy] (Gioanni \& Lamarche, 1985). ICMS in the anterior of architectonic $\mathrm{Cg} 1$ region (lying along midline) produced eye/periocular and nose movements (Hall \& Lindholm, 1974). 


\subsubsection{ICMS in AGM and PL evokes vibrissae movements}

ICMS in AGm (lying immediately lateral to Cg1) produces vibrissae movement.

However, this area has slightly higher thresholds than $\mathrm{Cg} 1$, especially when attempting to stimulate closer than $1 \mathrm{~mm}$ to bregma (Gioanni \& Lamarche, 1985). Coordinates to vibrissae motor cortex along AGm fall between 1.5 to $2.5 \mathrm{~mm}$ anterior to bregma and 1.0 to $2.5 \mathrm{~mm}$ lateral from midline (Brecht, et al., 2004a). AGm stimulation with $32 \pm 22 \mu \mathrm{A}$ $($ mean $\pm \mathrm{SD})$ within these coordinates produced vibrissae movements (Brecht, et al., 2004a). The number of vibrissae that moved varied frequently, and at times vibrissae movement occurred specifically along a single mystacial row. With sufficiently low thresholds, individual vibrissae movements were evoked. The degree of movement also varied with cortical depth and level of anesthesia, as both were independently responsible for increasing or decreasing vibrissae movement. Ipsilateral vibrissae movements were frequently identified by stimulating AGm, however this type of movement has yet to be studied in great detail. A second vibrissae region was also identified in the posteriorlateral (PL) motor cortex (Gioanni \& Lamarche, 1985), which has been shown to have lower threshold responses than any other region (Gioanni \& Lamarche, 1985). Although the extent of motor maps for the present studies were limited to the medial strip of vibrissae motor responses and lateral to forepaw responses, the vibrissae responses found in PL were used as a posterolateral border for motor maps. ICMS maps in the present study indicated that the PL was always separated from AGm by a hindpaw motor response. ICMS in posterior PL evoked movement from vibrissae row A, as well as from B, C, D, and E rows when ICMS was placed rostromedial to PL (Gioanni \& Lamarche, 1985). 


\subsubsection{Forelimb responds to ICMS in AGl}

Directly lateral to AGm is AGl, a cortical region primarily responsible for body, head, and paw movement (Brecht, et al., 2004a). In some studies the forelimb motor cortex also was divided into separate caudal and rostral zones similar to the medial and lateral vibrissae areas. Neafsey et. al (1986) described the rostral forelimb (RFL) and caudal forelimb (CFL) areas, with the RFL either continuing directly off the rostral tip of CFL, or separated with a small intervening cortical strip of neck or vibrissae movements (Neafsey, et al., 1986). The RFL was hypothesized to be a supplementary motor area similar to the secondary somatosensory cortex for vibrissae movement in rodents. RFL corresponds to the supplementary motor area of monkey and rat (Hall \& Lindholm, 1974). The rat RFL is much less responsive to sensory input from the periphery than CFL (Neafsey, et al., 1986). Previous studies on motor cortex also frequently revealed that RFL was highly dependent upon level of anesthesia. For example, to evoke a motor response in the RFL when anesthesia level was too high, a stimulating current greater than $100 \mu \mathrm{A}$ was needed, which was the limit of acceptable current in the present studies.

Finally, the motor cortex also contained regions that activated movement in the hindpaw, trunk, and tail. The MI regions that produced this movement were posterior to AGm, whereas lower-lip, tongue, and jaw movements were elicited when ICMS was placed anterior and/or lateral to RFL (Neafsey, et al., 1986).

Following ICMS mapping of MI, lesions were made at certain electrode penetration sites in order to match the ICMS maps with anatomical substrates and to identify the transition regions within cortex indicating the ICMS boundaries. The MI lesion studies showed that coronal cortical sections contained anatomically visible 
boundary zones between AGl, AGM, Cg1, granular, and dysgranular cortex (Neafsey, et al., 1986).

The SI to AGl boundary is defined morphologically by an abrupt transition in cortical layer thickness. (Donoghue \& Wise, 1982). This transition is $\sim 500 \mu \mathrm{m}$ wide and consists of a reduction in layer IV thickness with a concomitant increase in layer $\mathrm{V}$ thickness (Brecht, et al., 2004a) and is typical for transition regions between sensory and motor cortex (Kim \& Ebner, 1999).

Histological staining variations assist in differentiating between AGl and AGm regions as Thionin stains layer III of AGm only a pale color while layer II of AGl stains much darker (Donoghue \& Wise, 1982). Using immunohistochemistry the boundary between AGm and Cg1 is most clearly identified by the increase in layer I and layer II area thickness moving from AGm towards Cg1. A clear morphological boundary is present in the AGm to AGl transition as layer V cortex expands and layer III becomes less thick in AGl. Additional AGm/AGl differences relate to the homogeneous cellular appearance of superficial cortical layers in AGl as well as the less densely packed cellular layer II and thicker cortical layer V that also contains larger and more densely stained cells (Donoghue \& Wise, 1982). The transition between AGl and AGm can occur in less than $100 \mu \mathrm{m}$.

The area of cortical surface maps showed that body, head, and paw movements, produced by ICMS in AGl, was widest rostrally and tapered off to a narrow strip posteriorly. ICMS mapping also indicated that AGm surrounded the brain's frontal pole and was thinner than AGl. AGm traveled caudally along the dorsomedial portion of the hemisphere. Both AGm and AGl were very narrow as they reached the caudal limit of 
their posterior border where these agranular fields merged with the agranular retrospinal cortex (Donoghue \& Wise, 1982). The cytoarchitectonic features of rat motor cortex, when overlaid with ICMS, provide clear morphological and physiological comparisons similar to those found in SI.

In conclusion, rodent MI consists of three major features: 1) It has an agranular cytoarchitectonic appearance. 2) Using ICMS, a motor map of the periphery can be produced with stimulating currents less than $100 \mu \mathrm{A}$, while the average ICMS is $\sim 30 \mu \mathrm{A}$.

3) Using ICMS, the entire periphery is shown to be represented in MI.

\subsection{CORTICAL DEVELOPMENT IN NORMAL RATS}

\subsubsection{Pyramidal cell development}

In normal rats, telencephalic development of the pyramidal cells begins immediately prior to cortex formation and consists of postmitotic neurons gathering below the pial surface to form the preplate layer. Successive waves of neuronal generation occur in the subventricular and ventricular zones of the lateral ventricles (Lopez-Bendito \& Molnar, 2003). These newly generated neurons then travel along preestablished radial glia processes, with the assistance of glial growth factor. The cortical plate is then formed (Rakic, 2003), possibly by way of a stop signal (protein SPARC like1) given to migrating neurons and found predominately in upper cortical layer radial glial fibers (Gongidi, et al., 2004).

The cortical plate is then divided by thalamocortical afferents, into the superficial marginal zone (the future layer I) and deep subplate [future layers II to VI] (Lopez- 
Bendito \& Molnar, 2003). The ensuing development of normal rat cortex begins in the second gestational trimester with cortical neurogenesis occurring on gestational day (GD) 12 to 13 . On GD14 to GD16, daily neuronal generation is at its maximum where it is maintained until GD17 to GD19. Neuronal generation then decreases steadily from $\sim$ GD18 until GD21. PD0 marks the day that thalamocortical afferents have entered layers V and VI (Lopez-Bendito \& Molnar, 2003). Furthermore, by parturition, the majority of neuroblast and neuronal mitotic activity has ceased (Kaplan \& Hinds, 1977). By PD2 thalamocortical projections have entered layer IV and begun their organization into periphery-related patterns. By PD8, cortical layers I to VI are well formed. PD8 also marks the time point from which periphery-related activation of clearly defined layer IV barrels can be elicited by using voltage-sensitive dyes (Lopez-Bendito \& Molnar, 2003). Following a postnatal brain growth spurt, neuronal migration is usually complete by PD10 in rodents (Hicks \& D'Amato, 1968), which is analogous to the third trimester in human development (Dobbing \& Sands, 1979). The majority of cortical neuronal changes are finished by PD30 with the completion of apoptosis (Finlay \& Slattery, 1983; Friedman \& Price, 1986; Heumann, Leuba \& Rabinowicz, 1978).

Cortical generation thus follows a typical inside-to-outside sequence; i.e., deep layer VI is produced first with subsequent layers produced later. However, the gestational day of neuronal generation varies slightly across cortical regions. For example, layer VI neurons of lateral area 2 are generated between GD12 to GD13, whereas layer VI neurons of medial area 17 are generated on GD13 to GD16. It can thus be said that cortical neurogenesis occurs in a lateral-to-medial direction as demonstrated in humans (Chan, et al., 2002) mice (Smart \& McSherry, 1982) and rats (Catalano, Robertson \& 
Killackey, 1996; McCandlish, Waters \& Cooper, 1989; McCandlish, Li \& Waters, 1993) as well as in a rostrocaudal direction (Miller, 1988).

\subsubsection{Nonpyramidal cell development}

In rats, cells with typical features of Cajal-Retzius cells first gather in the ganglionic eminence of the subpallium/ventral telencephalon and begin their migration to the primordial plexiform layer or pre-plate at approximately GD14 (Parnavelas, 2002). This first migratory wave exits the medial ganglionic eminence (MGE) and primarily invades the pre-plate in a tangential direction. At GD15 to GD17 a second more prominent wave leaves the MGE and migrates to the intermediate zone (Metin, et al., 2006). In the later stages of corticogenesis, cells originating mostly in the lateral ganglionic emininence (LGE) and some from the MGE appear in the lower intermediate zone and the subventricular zone (Anderson, et al., 2001).

Using genetically manipulated rodent models such as D1x1 and D1x2 double mutant knock-outs, investigators have identified that these mutants have $75 \%$ fewer GABAergic interneurons in the cortex and that MGE appears to be the major source of these cortical interneurons (Metin, et al., 2006; Nadarajah \& Parnavelas, 2002;

Parnavelas, 2002; Stuhmer, et al., 2002). The LGE is the main source for interneurons migrating to the striatum and olfactory bulb (Wichterle, et al., 1999). These findings suggest that the majority of GABA-containing neurons in the mammalian cortex are derived from progenitors in the subpallium/ventral telencephalon. It is now understood that the migrating cells from MGE, that tangentially enter the pre-plate and intermediate zone, become the GABAergic interneurons of the cortex (Parnavelas, 2002). 
In contrast to the pyramidal cells, that use radial glia as a scaffolding to migrate out to the cortical layers, interneurons use cortico-fugal axons as a scaffold for migration to the cortex (Metin, et al., 2006). Using cortical slices, time-lapse imaging, and tracerlabeling techniques, investigators observed interneurons migrate tangentially out to the cortex (in either the marginal zone or intermediate zone), pause briefly then migrate out radially to their final position within cortical layers (Parnavelas, 2002; Wichterle, et al., 1999). It is thus widely believed that both pyramidal neurons and interneurons migrate into the cortical layers in an "inside-out" pattern (Metin, et al., 2006).

\subsection{TERATOGENIC EFFECT OF PAE ON DEVELOPING BRAIN}

\subsubsection{The effect of PAE on cortical neurogenesis during the first trimester (GD1 to GD12) in rodent}

Exposure of rodents to alcohol during the first trimester severely affects the microanatomy of the cerebral cortex. For example, ethanol exposure during GD9 to GD12 (Fakoya, 2005; Fakoya \& Caxton-Martins, 2006), a period that coincides with initial neuronal generation and migration (Miller, 1988; Zhou, et al., 2003), impaired astrocyte differentiation in the cerebral cortex. These results indicated that the cortical thickness of layer $\mathrm{V}$ was markedly diminished and consisted of pyknotic pyramidal neurons consisting mostly of "darkly stained collapsed cell bodies and folded membranes with broken apical dendrites" in juvenile rats as old as PD42 (Fakoya, 2005).

Furthermore, the few normal pyramidal neurons found in this layer were "nonviable as broken apical dendrites extended to upper cortical layers" (Fakoya, 2005) indicating that 
alcohol exposure during gestational day GD9 to GD12 can produce long-term effects on the cortical layers.

Acute PAE doses during the first trimester also have been shown to produce cortical disruptions. Ethanol administration to dams in 2 doses (25\% ethanol administered in two doses at $0.015 \mathrm{ml} / \mathrm{g}$, each separated by four hours) on GD8 resulted in significant volume reductions in neocortex, primary olfactory cortex, ventromedial and central forebrain structures with a concomitant total neuron number reduction at GD15 (Ashwell \& Zhang, 1996). However, the treatment with a single dose $(0.03 \mathrm{ml} / \mathrm{g})$ on GD8 showed no significant reductions in the same brain structures (Ashwell \& Zhang, 1996). These findings indicate that even very low ethanol doses could cause neuronal population deficits and that timing of the alcohol insult is important. Sufficient deficits in neuronal number in humans could lead to microcephaly, a common effect seen in children with FASD.

\subsubsection{The effect of PAE on cortical neurogenesis during the second trimester (GD12 to PD0) in rodent}

Cell culture studies revealed that exposure of primary neuritis from GD16 embryo to different alcohol concentrations $(450 \mathrm{nM}, 45 \mu \mathrm{M}, 45 \mathrm{mM})$ resulted in significantly lower cell numbers (Bingham, et al., 2004). Also, exposure of different groups of pregnant rats to different concentrations of $25 \%(\mathrm{w} / \mathrm{v})$ alcohol doses $(1.2-1.5,2.25,4.0$ or $6.0 \mathrm{~g} / \mathrm{kg})$ during the gestational period equivalent to the second trimester in humans showed that the effects on the brain were concentration dependent (Qiang, Wang \& Elberger, 2002). The highest concentration of ethanol in rat produced corpus callosum projection neurons 
with consistently "longer apical and basilar dendritic branches" in deep and superficial layers of visual cortex (Qiang, Wang \& Elberger, 2002). In addition, the brain weights of EtOH rats were significantly reduced. The mechanisms for increased dendritic projections following PAE is not known, but may be due to malfunctioned or disabled pruning mechanisms that would normally limit dendritic lengths. Similar disrupted pruning mechanisms may be an underlying factor involved with many of the studies by Miller (Miller, 1986; Miller, 1987; Miller, 1988; Miller, 1997) which suggest that a possible abnormal second wave of proliferation may occur in EtOH brains, resulting in more neurons in deep cortex and less neurons in superficial cortex when compared to control rats. An increase in dendritic corpus callosum arbor lengths may also be due to a loss of cholinergic neurons in the basal forebrain, as loss of cholinergic neurons reportedly increased dendritic length and number in human and rodent brains (Arendt, 1994; Qiang, Wang \& Elberger, 2002).

\subsubsection{The effect of first and second trimester PAE on cortical neurogenesis in rodent}

Rat models of PAE during the equivalent of the first two human trimesters [GD1 to GD20] (Dobbing \& Sands, 1973) resulted in more severely effected generation, proliferation, and migration of cortical neurons compared to PAE during either trimester

alone (Miller, 1986; Miller, 1988; Miller, 1993). Injection of $\left[{ }^{3} \mathrm{H}\right]$ thymidine into pregnant rat dams, followed by fetus or pup removal/sacrifice hour or days later, revealed that fetuses/pups exposed to gestational alcohol generated cortical neurons one day later 
while generation persisted two days longer than control pups. Similar retarded generation patterns were reported in corticospinal neurons (Miller, 1987).

Normally the most active time of neuronal generation is between GD13 to GD19, however, PAE pups had significantly fewer thymidine labeled neurons when injections occurred during GD13 to GD19. Interestingly, a late surge in neuronal proliferation and migration occurred in PAE rats after GD19. However, the late surge in neuronal migration patterns of PAE fetuses was not sufficient to fully supply cortical layer II with a normal concentration of cells. PAE also produced an unexpected increase in cell packing density, not seen in layers III to VI of non-PAE fetuses (Miller, 1986; Miller, 1988; Miller, 1993). At GD18, the ventricular thickness of the pups was reduced by $50 \%$ in both EtOH and control groups. However, between GD15 to GD20, subventricular zone thickness of PAE rats quadrupled and consistently became significantly thicker than the subventricular zone in control rats (Miller, 1989).

The difference in thickness between subventricular and ventricular zone following PAE may be due to an excitatory effect of ethanol at low concentrations (blood alcohol levels $\sim 165 \mathrm{mg} / \mathrm{dl}$ ) as studies have shown that the neurotrophins are higher in rats (Angelucci, et al., 1997; Miller, 2004; Moore, et al., 2004; Nakano, et al., 1996) following PAE. It is hypothesized that this differential effect may be caused by shielding of the subventricular zone by the ventricular wall of the ventricular zone (Miller, 1989). While high levels of alcohol may be found in the cerebral spinal fluid that comes in direct contact with the ventricular zone, the concentration of alcohol may be reduced in layers further away from the ventricular wall (Miller, 1989). The lower concentrations of alcohol may act to increase neuronal proliferation in the subventricular zone while 
possibly higher levels of alcohol at the ventricular zone may inhibit neuronal proliferation in zones that directly contact the ventricular wall (Miller, 1989).

$\left[{ }^{3} \mathrm{H}\right]$ thymidine studies have also revealed a second neuronal proliferative wave in the cortex of PAE rats, possibly pointing to a defect in apoptosis related pruning mechanisms (al-Rabiai \& Miller, 1989; Miller, Chiaia \& Rhoades, 1990). Gestational PAE has also produced defects in corpus callosum that were attributed to decreases in layers II, III, and V projection neurons, but increased neuronal distribution in layers V/VI (Miller, 1997). However, others were unable to find similar PAE-related gross defects on the corpus callosum (Livy \& Elberger, 2001). Evidence suggests that apoptotic pruning mechanisms may not reduce neurons in PAE brains if cell generation and migration occurs after active pruning mechanisms have completed their normal cycle of reducing neurons. If natural cell death does not reduce neurons in PAE animals, this may lead to an overabundance of late generating neurons (Rakic, et al., 1986).

\subsection{THE EFFECT OF PAE ON THE SENSORIMOTOR SYSTEM}

\subsubsection{Behavioral alterations in children with FASD}

Children with FASD have slower rates of interhemispheric transfer across the corpus callosum as well as a smaller corpus callosum (Roebuck, Mattson \& Riley, 2002). Studies on the motor performance of children with FASD showed that they overly relied on somatosensory input in performing motor response choice determination tasks (Conry, 1990; Janzen, Nanson \& Block, 1995; Roebuck, et al., 1998b; Wass, et al., 2002), had deficits in spatial fine motor control (Kaplan-Estrin, Jacobson \& Jacobson, 1999) as well 
as delayed fine motor skills (Kalberg, et al., 2006) when compared to children not exposed to alcohol in utero. A recent longitudinal study (Connor, et al., 2006) reexamined motor coordination and balance abilities of adults that had previously demonstrated PAE dose-dependent motor performance problems. Surprisingly, these adults no longer displayed the same dose-dependent motor problems (Connor, et al., 2006). Apparently, recovery from the teratogenic effect of PAE is possible in some regions of CNS, although three-quarters of adults with FASD continued to show motorfunction deficits that, as reported by Connor, were consistently but not significantly worse than control subjects (Connor, et al., 2006). Postural balance ability is also often deteriorated in children with FASD (Roebuck, et al., 1998a). Finally, reaction times (RT) are frequently associated with premotor and motor component deficits exhibited with FASD. In a study using simple RT involving only one hand, and the more complex choice RT using both hands, PAE children demonstrated slower CRT compared with controls and it was hypothesized that both central and peripheral mechanisms were affected (Burden, Jacobson \& Jacobson, 2005; Simmons, et al., 2002). Furthermore it was determined that the processing of sensory information was what slowed the RT of these children with FASD and not the actual motor response per se (Simmons, et al., 2006).

\subsubsection{CNS structural disruptions following PAE}

The most readily observable brain structural defect from PAE is microcephaly, defined as reduced head circumference and/or shrunken brain. Even after many facial dysmorphologies have subsided in time (Habbick, et al., 1998), microcephaly persists 
through adulthood (Streissguth, et al., 1991). Researchers have reported PAE-induced region specific changes in the brain and how these changes are dependent on alcohol concentration (Maier, et al., 1999; Maier, Miller \& West, 1999). In animal studies, an alcohol concentration of $6.5 \mathrm{~g} / \mathrm{kg}$ significantly reduced forebrain weights while lower concentrations ( 2.25 or $4.5 \mathrm{~g} / \mathrm{kg}$ ) had no effect (Maier, et al., 1999). In addition, $6.5 \mathrm{~g} / \mathrm{kg}$ alcohol produced no significant difference in the forebrain-to-body weight ratio, indicating proportional decreases in forebrain and body (Maier, et al., 1999). However, cerebellum-to-body weight ratios were significantly affected as PAE reduced cerebellar weight by $9 \%$ compared to control rats, suggesting that cerebellum may be particularly vulnerable to gestational alcohol exposure (Maier, Miller \& West, 1999).

The consumption of alcohol by a mother during gestation has been shown to severely affect the corpus callosum, the major fiber bundle connecting the two hemispheres of the brain. Investigators have reported a reduced/or thinned corpus callosum in humans; in extreme cases, PAE caused complete agenesis of the corpus callosum (Jeret, et al., 1987; Riley, et al., 1995). Fewer fibers crossing the corpus callosum may severely affect multiple brain regions. Normally, fibers initially projecting from parietal sensory cortex, caudate nucleus, and cerebellum, pass through the corpus callosum to contralateral motor and premotor areas (Bookstein, et al., 2002b). The effect of fewer callosal fibers synapsing in motor cortex may be responsible for some of the motor function deficits seen in children with FASD (Bookstein, et al., 2002b). Greater variability in callosal shape has been described in other studies (Bookstein, et al., 2002b); as well as a callosal shift in position relative to other CNS structures in FASD (Bookstein, et al., 2002a; Bookstein, et al., 2001). 
However, PAE affects more than the corpus callosum (e.g. cerebellum, cerebrum, and basal ganglia). PAE effects often occur in an asymmetrical fashion in which some brain regions are disproportionately reduced in comparison to surrounding regions. For example, PAE significantly reduced the cerebellum anterior lobules I to $\mathrm{V}$, while not significantly changing the size of posterior lobules VI to X (Sowell, et al., 1996). White matter volumes were more reduced (hypoplasia) than gray matter in the cerebrum. The parietal lobes were more affected than temporal and occipital lobes (Archibald, et al., 2001; Sowell, et al., 2002a). The parietal lobe was also disproportionately and additionally reduced in comparison to reductions of the entire brain (Riley \& McGee, 2005). Caudate nucleus was disproportionately reduced compared to the relatively unaffected hippocampus following PAE (Archibald, et al., 2001). Other brain structural alterations associated with FASD are reductions in the sizes of the cerebrum and cerebellum (Archibald, et al., 2001; Sowell, et al., 1996), basal ganglia, and diencephalon (Mattson, et al., 1996). Numerous imaging studies in children with FASD have been useful in elucidating the effect of PAE on the developing CNS. However, the use of animal models can also yield a plethora of information useful in further understanding CNS anatomical and functional defects caused by PAE.

\subsubsection{PAE sensorimotor disturbances in rodent}

Rodent PAE models have been used to identify many structural alterations in somatosensory and motor cortices to better understand the mechanisms for the behavioral and learning disruptions observed in children with FASD. PAE resulted in cortical ultrastructure abnormalities (Bingham, et al., 2004; Fakoya, 2004), and immature cell 
populations in the sensory and motor cortices (Popova, 1989). These immature cells showed signs of development delays when compared to non-PAE normally maturing cells at PD21, PD30 and PD60. Even at two months of age, PAE pups displayed "cortical cells of a hypoxic nature," or cells with a shriveled, fragmented appearance (Popova, 1989). Furthermore, motor cortex pyramidal cell dendrites had abnormally long, thin, entangled spines rather than the typical stubby-mushroom shaped spines (Chaudhuri, 2000; Galofre, et al., 1987; Popova, 1989).

PAE caused disruptions in layer V somatosensory ultrastructure. Local circuit neuron cisternae were haphazardly organized; twice the normal numbers of symmetric synapses were formed by local circuit neurons, and dendritic spines and shafts were reduced along with neuropil (al-Rabiai \& Miller, 1989; Miller, Chiaia \& Rhoades, 1990). In contrast, the space occupied by axons significantly increased in PAE rats (al-Rabiai \& Miller, 1989; Miller, Chiaia \& Rhoades, 1990). PAE also resulted in 30\% fewer synapses in frontal and motor cortex with the majority of reductions found in the molecular layer of motor cortex compared to controls (Adnams, et al., 2001; Chaudhuri, 2000; Inomata, Nasu \& Tanaka, 1987).

PAE reduced the sizes of PMBSF and FBS in neonatal rats. Anterior barrels were more reduced than posterior barrels (Margret, et al., 2006a; Margret, et al., 2005b). PAE also reduced neuronal cell number in mouse PMBSF barrels with a concomitant reduction in barrel area (Powrozek \& Zhou, 2005). In organotypic cultures of rat cerebral cortex, alcohol increased cell death and disrupted neuronal migration in developing cerebral cortex (Mooney \& Miller, 2003). 
Gestational alcohol exposure significantly affected cortical thickness (Miller \& Dow-Edwards, 1988). PAE also significantly reduced the cortical thickness of forelimb motor cortex, vibrissae motor cortex, PMBSF and FBS. However, only cortical layer V of area 3 and layer V of area 2 were significantly reduced (Miller \& Dow-Edwards, 1988).

Glucose utilization studies have been used to identify the metabolic activity levels of particular CNS regions. These studies showed significant decreases in metabolic activity of somatosensory and motor cortex in rats exposed to gestational alcohol (Miller \& Dow-Edwards, 1988), with the greatest decrease (29\%) in cortical layer IV (Miller \& Dow-Edwards, 1988). Further cortical metabolic activity was analyzed by observing glucose uptake in PAE and non-PAE rats. Results indicated that lateral cortical areas were more affected (less metabolically active) following PAE than were medial cortical areas, suggesting that SI of PAE rats should be more effected by alcohol than the more medially positioned MI cortex (Miller \& Dow-Edwards, 1988). Similar metabolic reductions have been identified in the principal sensory nucleus of the trigeminal nerve (Miller \& Dow-Edwards, 1993). In vitro slice preparations also indicated a reduction in neuronal excitability in layer II, III, and V, while layer IV was not examined (Sessler, et al., 1998).

Other investigators examined the effect of PAE on SI and MI. PAE not only delayed cortical neurogenesis by one to two days, but also prolonged neurogenesis by one to two days (Miller, 1988). PAE may also disrupted apoptotic pruning mechanisms, as the total number of corticospinal neurons was significantly increased in rats exposed to 
gestational alcohol (Miller, 1987). A similar effect occurred in neurons that projected via corpus callosum (Miller, 1997).

\subsection{TERATOGENIC EFFECT OF ALCOHOL ON HUMAN BRAIN AND BEHAVIOR}

Children with FASD frequently show signs of microcephaly (Archibald, et al., 2001; Clarren, et al., 1978; Lemoine, 1992; Riley, McGee \& Sowell, 2004), disruptions in the morphology of the corpus callosum (Bookstein, et al., 2002b), corpus callosum agenesis (Jeret, et al., 1985), cortical heterotopias (Guerri \& Renau-Piqueras, 1997), and defects in the commissures. Children with FASD often also show anomalies in cerebellum (O'Hare, et al., 2005), hippocampus (Riikonen, et al., 1999), brainstem, and basal ganglia (Mattson, et al., 1996); all of which have been previously reviewed in numerous papers (Archibald, et al., 2001; Guerri, 1998; Riley \& McGee, 2005; Riley, McGee \& Sowell, 2004; Roebuck, Mattson \& Riley, 2002). These brain anomalies often culminate in life-long social and behavioral difficulties.

Children and adults with FASD may exhibit numerous forms of cognitive and behavioral disorders that vary in severity. Behavioral and cognitive disorders may include the following: disruptions in fine motor control (Connor, et al., 2006; Janzen, Nanson \& Block, 1995; Kalberg, et al., 2006; Kaplan-Estrin, Jacobson \& Jacobson, 1999; Roebuck, et al., 1998b), increased reaction and choice reaction times (Simmons, et al., 2002), disruptions in higher-order cognitive competency (Adnams, et al., 2001), hyperactivity (Burd, et al., 2003b), language disorders (Church, et al., 1997), as well as auditory and visual inattention of varying degrees (Connor, et al., 1999; Kable \& Coles, 2004; 
Stromland, et al., 2005). Disordered behavior (Hoyme, et al., 2005), impaired place learning (Hamilton, et al., 2003), poor academic performance, trouble with the law, and inappropriate sexual behavior (Kelly, Day \& Streissguth, 2000) are also outcomes in children with FASD. Cognitive disruptions may occur in learning and memory (Richardson, et al., 2002), memory for location (Stromland, et al., 2005), low IQ scores (Bailey, et al., 2004; Streissguth, Barr \& Sampson, 1990), and impaired number processing (Burden, Jacobson \& Jacobson, 2005; Kopera-Frye, Dehaene \& Streissguth, 1996) especially when processing increasing amounts of information (Simmons, et al., 2006). Delayed processing of information (Kable \& Coles, 2004; Olson, et al., 1998), deficits in verbal learning, inability to maintain complex attention, poor executive functioning (Connor, et al., 2000; Kerns, et al., 1997), and an increase in mental illness (Famy, Streissguth \& Unis, 1998), depression, and suicide (Kelly, Day \& Streissguth, 2000) have also been reported in children with FASD. The culmination of any number of these behavioral and cognitive disruptions may lead to massive atypical executive and adaptive functioning in children with FASD (Riley \& McGee, 2005).

However, while the teratogen is alcohol, it alone is not sufficient to produce the effect seen in children with FASD. The internal and external environments of the mother and fetus also contribute to the severity of symptoms in a child with FASD (Stratton, Howe \& Battaglia, 1996).

\subsubsection{Factors contributing to the effect associated with FASD}

While numerous studies (Abel, 1984a; Abel, 1984b; Abel \& Hannigan, 1995; Chiriboga, 2003; Church \& Abel, 1998; Covington, et al., 2002; Fox, Sexton \& Hebel, 
1990; Haorah, et al., 2005; Hebel, Fox \& Sexton, 1988; May, et al., 2005; NordstromKlee, et al., 2002; Primatesta, et al., 1993; Stromland, et al., 2005; Viljoen, et al., 2001) have detailed the many environmental, behavioral, and biological factors that are relevant to a study dealing with the effect of PAE on children with FASD (culture/ethnicity of mother, comorbidity of smoking, genetic susceptibilities, blood brain barrier) that are relevant to a study of children with FASD. The important factor for this dissertation deals with the pattern of alcohol exposure.

FASD generally only occurs in children of alcohol abusing mothers. Abusive or heavy drinking patterns in humans has been defined by the U.S. Substance Abuse and Mental Health Services Administration as the consumption of five drinks per occasion at least five times a month (SAMHSA, 1996, p. 91) and by the National Institute of Alcohol Abuse and Alcoholism as two or more standard drinks a day (NIAAA, 1993). Generally, heavy/abusive drinking is understood as a minimum of five standard drinks consumed over a two hour period once a week, while gestational blood alcohol levels (BAL) of $\sim 150 \mathrm{mg} / \mathrm{dl}$ (Abel, 1998; Frezza, et al., 1990) are typically more accurate than the number of drinks in determining possible FASD symptoms. To achieve these high BALs, mothers often consume alcohol in a binge-type drinking pattern.

Human and animal studies have shown that chronic bingeing is highly correlated with more severe deficits than when equivalent amounts of alcohol are spread out over time (Bonthius, Goodlett \& West, 1988; Rosett, et al., 1983). The higher BALs from bingeing push a fetus towards a biological "threshold" for FASD-type patterns to occur (Abel \& Hannigan, 1995; Bonthius \& West, 1988; Rosett, et al., 1983; West, et al., 1990). Clinical examinations have shown that newborns of chronic binge drinking 
mothers have more severely affected facial anomalies (Clarren, Astley \& Bowden, 1988) and increased impairments of cognitive functioning (Streissguth, et al., 1989). Prolonged BALs are more likely to influence certain events during critical periods of development (Maier \& West, 2001a). However, while chronic bingeing most readily produces FASD symptoms, moderate PAE can also produce deficits in humans (Sood, et al., 2001) and

animals (Savage, et al., 2002; Zhou, et al., 2003). Although low BAL seldom produces an FASD effect, a low BAL has a greater potential to produce FASD symptoms when combined with multiple extraneous factors such as low socioeconomic status (SES), illhealth, poor nutrition, smoking, culture, and genetic deficits (May, et al., 2004). These factors are understood to exacerbate the effect of PAE on the developing fetus.

Although each of the above factors likely exacerbate the symptoms associated with FASD, the experiments presented in this dissertation are primarily concerned with: 1) producing BALs sufficiently high to be comparable to chronic binge-type drinking patterns in humans; 2) administering alcohol between GD1 to GD20, as these time points are comparable to human first and second trimester exposure (Dobbing \& Sands, 1973); and 3) controlling the effect of stress and nutrition in relation to PAE.

\subsubsection{Mechanisms mitigating brain deficits following PAE}

Following PAE in humans, numerous brain deficits have been identified including size, shape, and neuronal number from many CNS regions such as: cerebellum, hippocampus, temporal, occipital, and parietal cortices (Archibald, et al., 2001; Riikonen, et al., 1999; Riley, McGee \& Sowell, 2004; Sowell, et al., 2001; Sowell, et al., 2002b). Similar brain deficits have also been identified in animal models. These models have 
greatly enhanced our understanding of PAE-related effects in brain and are critically important for identifying new treatment strategies for ameliorating FASD symptoms in humans. Many factors, either singly or in combination, are attributed to PAE teratogenicity and brain damage due to the toxic effect of alcohol on the brain. PAE can disrupt fetal development by: reducing antioxidant levels such as ascorbic acid (Vitamin C), $\alpha$-tocopherol (Vitamin E), and $\beta$-carotene (Chen, Dehart \& Sulik, 2004; Goodlett, Horn \& Zhou, 2005; Wu \& Cederbaum, 2003); reducing blood flow across placenta thus reducing glucose nutrients to the fetal brain and body (Falconer, 1990; Jones, Leichter \& Lee, 1981); disrupting DNA repair mechanisms (Couch \& Baker, 2002; Lamarche, et al., 2003); decreasing glucose uptake (Singh, Snyder \& Pullen, 1989); inhibiting neuroprotective proteins which can thereby lead to increased levels of apoptosis (Bhave \& Hoffman, 1997); and inhibiting retinoic acid metabolism (Duester, 1991; Pullarkat, 1991).

Serotonin (5-HT) plays a critical role in the development of brain (Sari, Powrozek \& Zhou, 2001), therefore the effect of PAE on 5-HT has been studied in an attempt to link FASD and particularly ARND microcephaly with 5-HT disruption. Studies have indicated that PAE reduces 5-HT stained fibers in the diencephalon and septal nuclei and in cingulate, frontal, subicular, and parietal cortices (Sari, Powrozek \& Zhou, 2001; Zhou, Sari \& Powrozek, 2005; Zhou, et al., 2001). PAE also reduced 5-HT stained fibers in dorsal hippocampus CA4, cinereum, caudate-putamen, and hypothalamus (Zhou, et al., 1994a) as well as ascending projection fibers to forebrain, and dorsal raphe and median raphe nuclei (Zhou, et al., 1994b). These results suggest that low levels of serotonin may be partially responsible for the microcephaly seen in children of mothers who drank 
alcohol heavily during pregnancy. Although the effect of PAE on the above intrinsic factors may assist in perturbing normal CNS development, excessive generation of molecules called "free radicals" plays a pivotal role in PAE-induced oxidative stress (OS) and subsequent CNS damage (Goodlett \& Horn, 2001; Wu \& Cederbaum, 2003) and because of their key role, free-radicals will be discussed more fully below.

\subsubsection{PAE and oxidative stress in the developing CNS}

Particularly detrimental to CNS development are the degradation of lipids, proteins and DNA induced by oxygen containing free radicals known as reactive oxygen species [ROS] (Goodlett \& Horn, 2001; Goodlett, Horn \& Zhou, 2005; Heaton, Mitchell \& Paiva, 2000; Kumral, et al., 2005; Peng, et al., 2005; Taylor \& Crack, 2004). Ethanol exposure has been shown to produce cell death by way of oxidative stress (OS) in both human (Davis \& Syapin, 2004; Rao, et al., 1996) and animal (Chen, Dehart \& Sulik, 2004; Heaton, et al., 2003; Reinke, et al., 1987) studies. OS has been implicated in DNA fragmentation (Ames \& Saul, 1986; Halliwell, 1987), protein disruption, lipid peroxidation of biological membranes (Montoliu, et al., 1994), and mitochondrial dysfunction (Goodlett \& Horn, 2001). Also, OS can produce cellular changes associated with neurodegeneration and structural defects in developing fetal brains by breaking down the membranes of lipid dense neurons (Kumral, et al., 2005; Marino, Aksenov \& Kelly, 2004; Ramachandran, et al., 2001).

During development, brain cells naturally undergo a process of apoptosis. However, alcohol-induced elevation of free radicals may enhance apoptosis causing greater cell death. Therefore, an increase in free radicals by PAE may promote abnormal 
formation of brain structures as a result of disruptions in cell maturation, proliferation and migration. The effect of ethanol on the production of free radicals, how free radicals affect the brain, how alcohol may change brain morphology through free radical production, and finally why the developing brain is more susceptible to free radicals compared with other regions of the body are described in sections 1.9.3.1 to 1.9.3.3.

\subsubsection{Generation of ROS from aldehyde, the by-product of ethanol metabolism}

The metabolism of alcohol is linked to the induction of OS (Zima, et al., 2001) via conversion of alcohol into acetaldehyde (a toxic and reactive molecule) by the enzyme alcohol dehydrogenase in the kidneys. Next, in the presence of the aldehyde dehydrogenase enzyme, acetaldehyde is converted to acetate. The metabolism of alcohol through chemical reactions increases the production of nicotinamide adenine dinucleotide (NADH) molecules which are involved in ATP production via the mitochondrial respiratory chain (Wu \& Cederbaum, 2003). During the creation of ATP in the mitochondria, ROS are formed as intermediate or transition products. Although normal physiologic levels of ROS are rapidly converted into water via electron and proton reactive processes, excessive induction of ROS by high BALs cannot be completely eliminated by antioxidant enzymes in the body. Therefore, ROS from excessive alcohol consumption during pregnancy can enhance neurodegeneration, thereby facilitating cell death in the developing brain (Wu \& Cederbaum, 2003). 


\subsubsection{Additional ethanol-induced ROS production and cell death}

The ethanol-inducible enzyme cytochrome P-4502E1 (CYP2E1) is a by-product of ethanol metabolism (Sun, et al., 2001), and is of great interest for alcohol research particularly during brain development. This enzyme is distributed widely in the brain (Montoliu, et al., 1995) and is significantly increased by heavy alcohol exposure (Lieber, 1997). Since CYP2E1 induces lipid peroxidation, or the dissolving of a cell's lipid membrane (Montoliu, et al., 1994), alcohol induced-elevation of the enzyme may be detrimental to brain cells during their generation, proliferation, and migration. Lipid peroxidation has been shown to decrease when ethanol-incubated brain homogenates were treated with a CYP2E1 specific antibody (Montoliu, et al., 1994) indicating CYP2E1's role in lipid peroxidation.

Additional sources of alcohol induced ROS damage may occur by way of: 1) increased blood pressure followed by endothelium-mediated vasodilation and subsequent blood flow disruption. This disruption triggers xanthine oxidase production which is an important dehydrogenase that assists in NADH and ROS production (Puddey, et al., 2001); and 2) decreased superoxide dismutase (SOD) enzyme, which eliminates ROS. Gestational ethanol exposure for four weeks produced BALs between $150 \mathrm{mg} / \mathrm{dl}$ to 350 mg/dl (Polavarapu, et al., 1998) and decreased SOD levels (Nanji \& Hiller-Sturmhofel, 1997), suggesting that ethanol may facilitate cell death via reduction of SOD antioxidants. Furthermore, ethanol decreased the level of the antioxidant vitamin E, which in turn increased the levels of damaging ROS (Nanji \& Hiller-Sturmhofel, 1997). 


\subsubsection{ROS produces cell death}

When ROS levels are elevated, oxidant and antioxidant equilibrium is disturbed; thus, the antioxidant enzymes in the body are inadequate to completely eliminate ROS from body systems. Therefore, high levels of ROS make lipid peroxidation and cell death unavoidable. Lipid peroxidation is the last phase of cell death and occurs as the lipid membranes of organelles within the cell and the cell wall itself becomes liquefied and bursts. This bursting of cell membranes is devastating to an already imbalanced system, as the ruptured cell membranes release their contents, typically consisting of numerous metals and chelated ROS, further destabilizing surrounding cells (Halliwell, 1987). It is likely that mitochondria are key players in lipid peroxidation induced cell-death as described below.

Mitochondria are the powerhouse structures behind cellular ATP production and the main source of an animal's energy supply; and normal mitochondrial structures are critical for lipid membrane maintenance and cell survival. Following lipid peroxidation of the mitochondrial cell wall, large holes develop in the mitochondrial membrane allowing release of $\mathrm{Ca}+$ and cytochrome $\mathrm{C}$. The release of Cytochrome $\mathrm{C}$ activates caspases, while the excess $\mathrm{Ca}+$ is excitotoxic (Choi, 1995; Goodlett \& Horn, 2001; Kroemer, Zamzami \& Susin, 1997); indicating that lipid peroxidation of mitochondrial walls causes apoptosis and necrosis. This supports the evidence that generation of superoxide free radicals occurs through an ethanol induced lesion in the mitochondrial respiratory electron transport chain (Bailey, Pietsch \& Cunningham, 1999; Sun, et al., 2001). 


\subsection{HUMAN VERSUS ANIMAL BLOOD ALCOHOL LEVEL}

Animal models have been useful in correlating BALs with behavioral and structural alterations that relate back to human FASD symptoms. However, caution must be taken when attempting to relate animal results directly to humans, as animals often require extremely high BALs to elicit a teratogenic effect similar to those seen in FASD children. Incidentally, these differences are often due to variations in the rate of alcohol absorption, metabolism, distribution, and elimination between species. For example, to achieve a $3 \mu \mathrm{g} /$ liter blood level for some drugs requires that rabbits be administered with $200 \mathrm{mg} / \mathrm{kg}$ while humans require only $1 \mathrm{mg} / \mathrm{kg}$ (Abel, 1998). Therefore the concentration of alcohol in the blood is the most accurate measure when comparing the effect of PAE in animals and humans, as BAL is generally the same concentration in the blood as at alcohol's site of action (Abel, 1998).

In the present studies, a PAE rat model, binged daily with $6 \mathrm{~g} / \mathrm{kg}$ was used to achieve BALs measured at $\sim 300 \mathrm{mg} / \mathrm{dl}$. This BAL is the human equivalent of 27 drinks/day or 15 drinks in a 5-hour period in a man weighing $130 \mathrm{lbs}$. The equivalent of $a$ drink is defined as 12 ounces of beer, 5 ounces of wine, or 1.5 ounces of 80-proof distilled spirits. Therefore, the human equivalent to the dosage paradigm used in this dissertation research would be $324 \mathrm{oz}$ of beer/day, $135 \mathrm{oz}$ of wine/day or $40.5 \mathrm{oz}$ of hard liquor/day. These "drink equivalents" are related to gender and one less drink per every 10 drinks (Abel, 1998) would properly equate for women. An additional example to relate this dissertation's rat model exposure level to humans would state that while the legal driving limit of $0.08 \mathrm{~g} / \mathrm{dl}$ equates to $80 \mathrm{mg} / \mathrm{dl}$ on the scale reported here, rat BALs measured in these studies were $\sim 300 \mathrm{mg} / \mathrm{dl}$. 
However, the relationship between rodent and human BALs is difficult to surmise since $6 \mathrm{~g} / \mathrm{kg} /$ day frequently left dams in a state of immobility for several hours. Because non-alcoholic humans seldom achieve BALs in the $300 \mathrm{mg} / \mathrm{dl}$ level, due to the immobility such BALs typically produce, it would be difficult to achieve such levels in the general population. Unconsciousness would usually transpire in human females prior to reaching a BAL of $\sim 300 \mathrm{mg} / \mathrm{dl}$ as used in our rat studies. However, some human individuals have survived BALs as high as $500 \mathrm{mg} / \mathrm{dl}$ to $780 \mathrm{mg} / \mathrm{dl}$ (Lindblad \& Olsson, 1976). On the other hand, while a BAL of $260 \mathrm{mg} / \mathrm{dl}$ is the beginning level for lethality in most people (Abel, 1998), the tolerance level for pregnant alcoholics may permit such a high BAL without death. Our rat model required high BALs due to the aforementioned species differences in alcohol metabolism, etc. 


\section{CHAPTER 2}

\section{THE LONG-TERM EFFECT OF PRENATAL ALCOHOL EXPOSURE ON POSTEROMEDIAL BARREL SUBFIELD IN JUVENILE AND ADULT RATS}

\subsection{INTRODUCTION}

Prenatal alcohol exposure (PAE) can result in children with growth retardation, craniofacial abnormalities, and central nervous system dysfunction and has led to the clinical diagnosis of fetal alcohol syndrome [FAS] (Jones \& Smith, 1973; Jones \& Smith, 1975). Furthermore, children of mothers that consumed alcohol during pregnancy may not meet all of the criteria for a diagnosis of FAS, but nonetheless suffer from permanent damage to brain structures resulting in a variety of dysfunctions including deficits in central nervous system processing. The term, fetal alcohol spectrum disorder (FASD), has been reserved to describe the range of deficits resulting from early alcohol exposure (Goodlett, Horn \& Zhou, 2005; Riley \& McGee, 2005).

PAE children often have sensorimotor processing deficits as demonstrated by their poor performance on higher-order cognitive motor tasks (Adnams, et al., 2001), difficulties in maintaining postural balance (Roebuck, et al., 1998b), slower premotor and motor reaction times (Simmons, et al., 2002), and deficits in fine motor control (Connor, et al., 2006), and coordinated movement timing (Wass, et al., 2002). It has been reported that sensorimotor disturbances might be due, in part, to alterations in visual and auditory capacities (Church, 1987; Church \& Abel, 1998; Stromland, 1985; Stromland, 2004), reduction in overall brain size and shape (Roebuck, Mattson \& Riley, 1998; Sowell, et al., 2001), corpus callosum thinning (Riley, et al., 1995), reductions in parietal cortex 
volume (Archibald, et al., 2001) and basal ganglia volume, as well as a reduction in the area of the caudate-putamen (Mattson, et al., 1994).

Many of the sensorimotor deficits seen in children with FASD are mirrored in PAE animal models. The use of these models may therefore be helpful in uncovering potential deficits that can be further explored in children with FASD. Similar to children, animals exposed to prenatal alcohol may also exhibit impaired balance (Kelly, Hulsether \& West, 1987), disrupted righting reflexes (Lopez-Tejero, et al., 1986), delayed motor development (Molina, et al., 1987; Norton, et al., 1988), and altered corticospinal development (Miller, 1987). Furthermore, PAE has been shown to result in specific deficits in cerebellum (Maier, et al., 1999; Maier, Miller \& West, 1999; Maier \& West, 2001b), locus coeruleus (Maier \& West, 2003), and somatosensory cortex (Margret, et al., 2005a; Margret, et al., 2005b; Miller \& Potempa, 1990; Mooney \& Miller, 1999; Powrozek \& Zhou, 2005).

We (Margret, et al., 2006a; Margret, et al., 2006b; Margret, et al., 2005b), and others (Powrozek \& Zhou, 2005), have used the rodent barrel field cortex to study the effect of gestational alcohol exposure on the organization of somatosensory cortex. Clusters of cells, called barrels, located within layer IV of the primary somatosensory cortex (SI) are associated with the representation of the body surface. These barrels are readily amenable to quantitative methods that allow comparisons of area measurements between alcohol and non-alcohol groups. One subfield of barrels, the posterior medial barrel subfield (PMBSF) is associated with the representation of the large mystacial vibrissae on the contralateral face. Recently, Powrozek and Zhou (2005) examined serotonin labeling in mice at postnatal day 7 (PD7), and reported that PAE reduced the 
overall size of serotonin-labeled barrels in the PMBSF as well as the sizes of some of the individual barrels within the PMBSF. Furthermore, PAE not only reduced the number of cells within selected barrels, but in some cases, entire barrels were missing. Similarly, in PD9 rats, PAE reduced the size of the overall PMBSF, along with the sizes of individual barrels and septal areas lying between barrels, but did not alter barrel pattern or result in missing barrels. Furthermore, an asymmetry between posterior and anterior PMBSF barrels occurred as anterior barrels were more reduced in area than were the areas of posterior barrels compared to respective barrels in CF (Margret, et al., 2005b).

Several peripheral asymmetries are reported in rodents. For example, posteriorly and anteriorly located mystacial vibrissae differ in: vibrissae length and thickness (Brecht, Preilowski \& Merzenich, 1997), whisking behavior (Carvell \& Simons, 1990), vibrissae sensory encoding strategies (Ahissar \& Arieli, 2001), and innervation patterns to the mystacial pad (Welker \& Van der Loos, 1986) suggesting that not all vibrissae are alike. These peripheral asymmetries may lead to morphological barrel asymmetries, as barrel development in neonates is highly dependent on tactile sensory input (Waters, McCandlish \& Cooper, 1990). Studies have shown that immunohistochemical staining characteristics of barrel morphology can be modified by removing sensory input in neonatal (Van der Loos \& Woolsey, 1973; Waters, McCandlish \& Cooper, 1990), but not in adult rats (Wong-Riley \& Welt, 1980). However, no one has studied the role of sensory experience on cortical barrel development in PAE rats.

In the present study, we tested the hypothesis that PAE continues to exert longterm effects on the vibrissae barrel cortex by examining the PMBSF area, PMBSF barrel area, PMBSF septal area and areas of individual barrels observed in six-week-old 
(juvenile) rats and seven-month-old (adult) rats. We also tested the hypothesis that differential sensory experience may underlie the anterior-to-posterior asymmetry reported in neonatal rats and in the present study in juvenile and adult animals. Our results suggest that PAE continued to exert a long-term reduction in PMBSF area and PMBSF barrel area while leaving the barrel pattern unperturbed. Furthermore, our evidence from unilateral trimming studies did not support the hypothesis that differential sensory experience accounted for the anterior-to-posterior asymmetries reported in juvenile and adult rats.

\subsection{METHODS}

\subsubsection{Animals}

Out of a total of 136 Sprague-Dawley pups used in this study, 84 were used for the 6-week-old (juvenile) rat experiments, 25 were used for the 7-month-old (adult) rat experiments, and 27 were used for the 6-week-old vibrissae trimming experiment. Virgin female rats were bred overnight with adult male rats. All females were handled and habituated to the dry gavage procedure 4 days prior to breeding.

\subsubsection{Breeding and treatment groups}

Breeding occurred by placing 2 to 3 adult female rats in a cage overnight with an adult male breeder. Vaginal smears were examined the next morning under a microscope. Sperm positive slides were marked as gestational day one (GD1). At that time, pregnant females were weighed, and separated into one of three groups: alcohol $(\mathrm{EtOH}, \mathrm{n}=14)$, 
pairfed $(\mathrm{PF}, \mathrm{n}=10)$, and chowfed $(\mathrm{CF}, \mathrm{n}=13)$. PF mothers were matched in weight to EtOH mothers. Pregnant females were separated into individual cages during pregnancy and pup rearing.

\subsubsection{Treatment procedures}

The EtOH group was gavaged with 25\% (w/v) ethanol and water solution at a 6 $\mathrm{g} / \mathrm{kg}$ dose from GD1 to GD20. This period is equivalent to the combined first and second trimesters in humans (Dobbing \& Sands, 1973; Dobbing \& Sands, 1979). The PF group was gavaged with $25 \%$ maltose dextran and water solution that was isocaloric and isovolumetric to the alcohol given the EtOH group. PF mothers received only the amount of food consumed a day earlier by a previously matched EtOH female. The PF group served as a control for nutrition and stress. The CF group served as a normal control with no diet restriction or gavage treatment (excepting for the initial gavage acclimation given to all females). For the 6-week-old rat experiment, a cross-foster group (XF) was included to test for a maternal rearing effect. These pups received equal prenatal treatment as the EtOH group, however, following delivery, pups were fostered to a $\mathrm{CF}$ mother whose own recently delivered pups had been removed. For the vibrissae trimming study, the EtOH dams were first habituated to PAE by receiving $1 \mathrm{~g} / \mathrm{kg}$ on GD1, $3 \mathrm{~g} / \mathrm{kg}$ on $\mathrm{GD} 2,5 \mathrm{~g} / \mathrm{kg}$ on GD3 and $6 \mathrm{~g} / \mathrm{kg}$ from GD4 to GD20. All groups were provided with water ad libitum. 


\subsubsection{Daily procedures}

Food hoppers were removed from all groups daily between 0830 and 0930 hours. Daily dam weight and food consumption were recorded. Three hours following food hopper removal, EtOH and PF dams were gavaged with their respective solutions and food hoppers were returned to all groups. Stainless-steel gavage needles, lubricated with canola oil, were used on all dams prior to breeding to accustom virgins to the procedure. On GD1, dams were divided into their respective groups. EtOH and PF dams were then gavaged with plastic needles (Instech Solomon) while CF dams were no longer gavaged. The gavage procedure occurred at the end of a 3-hour fast in order to maximize solution absorption. Each day, EtOH and CF dams were given ad libitum access to chow food (Harlan).

\subsubsection{Blood alcohol level}

Blood samples were collected on GD13 and GD20 (at 60, 120, 180, and 240 minutes post-gavage) from EtOH and PF dams. Although blood samples were taken from $\mathrm{PF}$ dams (to control for the stress of handling the EtOH dams during gavages and blood sampling procedure) the PF samples were not analyzed for BAL. Blood was taken by placing the tail in warm water for 5 seconds, wiping off excess water with a paper towel and cutting the tail tip with a sharp razor blade. Blood from EtOH dams was collected in $20 \mu 1$ heparinised tubes, centrifuged to separate plasma, and stored at $4^{\circ} \mathrm{C}$. In contrast, blood samples were collected on GD10 and GD17 (at 60, 120, 180, and 240 minutes post-gavage) from EtOH dams whose pups received vibrissae trimming. Blood alcohol level (BAL) was measured using an AM1 alcohol analyzer (ANALOX). 


\subsubsection{Parturition and rearing}

Gavage treatment continued until GD20, at which time dams were transferred to clean cages. Two nesting pads were added to the cage. Other than a daily weight gain measurement, dams were left undisturbed until parturition, with free access to chow and water. Starting on GD22, cages were checked twice daily at 0800 and 1700 hours until delivery. On the day of birth (designated postnatal day 0: PD0), litters were culled to 8 pups. Pups were weaned from their mothers on PD28, separated into sex as well as litter specific cages where they remained with ad libitum food and water until sacrifice during the sixth postnatal week.

\subsubsection{Unilateral vibrissae trimming}

On postnatal day (PD) 5, pups in the $\mathrm{EtOH}(n=14)$ and the $\mathrm{CF}(n=13)$ groups were randomly assigned to have the vibrissae from either their left or right mystacial pads trimmed. Trimming began on PD5 and continued daily until PD42. In infraorbital nerve transection studies, PD5 was the earliest time point that the removal of vibrissae input did not permanently alter barrel field development (Boylan, et al., 2001; Shoykhet, Land \& Simons, 2005) Therefore, it was understood that vibrissae trimming that began on PD5 would still allow barrel field development to occur. Iridectomy scissors were used to evenly trim the posterior six arcs of mystacial vibrissae immediately above the skin surface.

Treatment groups were as follows: EtOH-trim = the cortical hemisphere contralateral to vibrissae trimming was used to test the combined effect of PAE and vibrissae trimming $(n=14)$; EtOH-intact $=$ the ipsilateral cortical hemisphere in EtOH 
group was used as the control for contralateral vibrissae trimming $(n=14)$. CF-intact $=$ the cortical hemisphere ipsilateral to mystacial pad trimming in CF pups that represented the intact, non-trimmed, vibrissae pad on the contralateral hemisphere was used to the control for contralateral vibrissae trimming $(n=13)$; $\mathbf{C F}$-trim $=$ the cortical hemisphere contralateral to mystacial pad trimming in CF rats $(n=13)$. The CF group received unilateral vibrissae trimming to control for postnatal experience differences due to handling of pups, possible increased stress levels during trimming procedure, and most importantly as a control for possible effects on the hemisphere contralateral to trimming in the absence of PAE. Two examples of trimmed and un-trimmed mystacial pads are shown in Fig 2.1.

All animals were maintained in the animal facility at The University of Tennessee

Health Science Center (UTHSC) throughout the experiments. They were kept in rooms at 78 to $80{ }^{\circ} \mathrm{F}, 35$ to $40 \%$ humidity, and a 12 -hour light/dark cycle. Experiments conformed to the Principles of Laboratory Animal Care (NIH publication No. 86-23, revised 1985) and were approved by the Animal Care and Use Committee, UTHSC. The Animal Care Facility is accredited by AAALAC.

\subsubsection{Tissue processing}

Juvenile rats in their sixth postnatal week and adult rats at seven months of age were weighed and given a lethal injection of Nembutal (100 mg/kg, i.p.). Rats were perfused intracardially with $0.9 \%$ saline followed by $4 \%$ paraformaldehyde in $0.15 \mathrm{M}$ sodium phosphate buffer saline (NaPBS, ph $\left.7.4,21^{\circ} \mathrm{C}\right)$. Their brains were removed from skulls and weighed (whole brain), then olfactory bulbs and cerebellum were removed and 

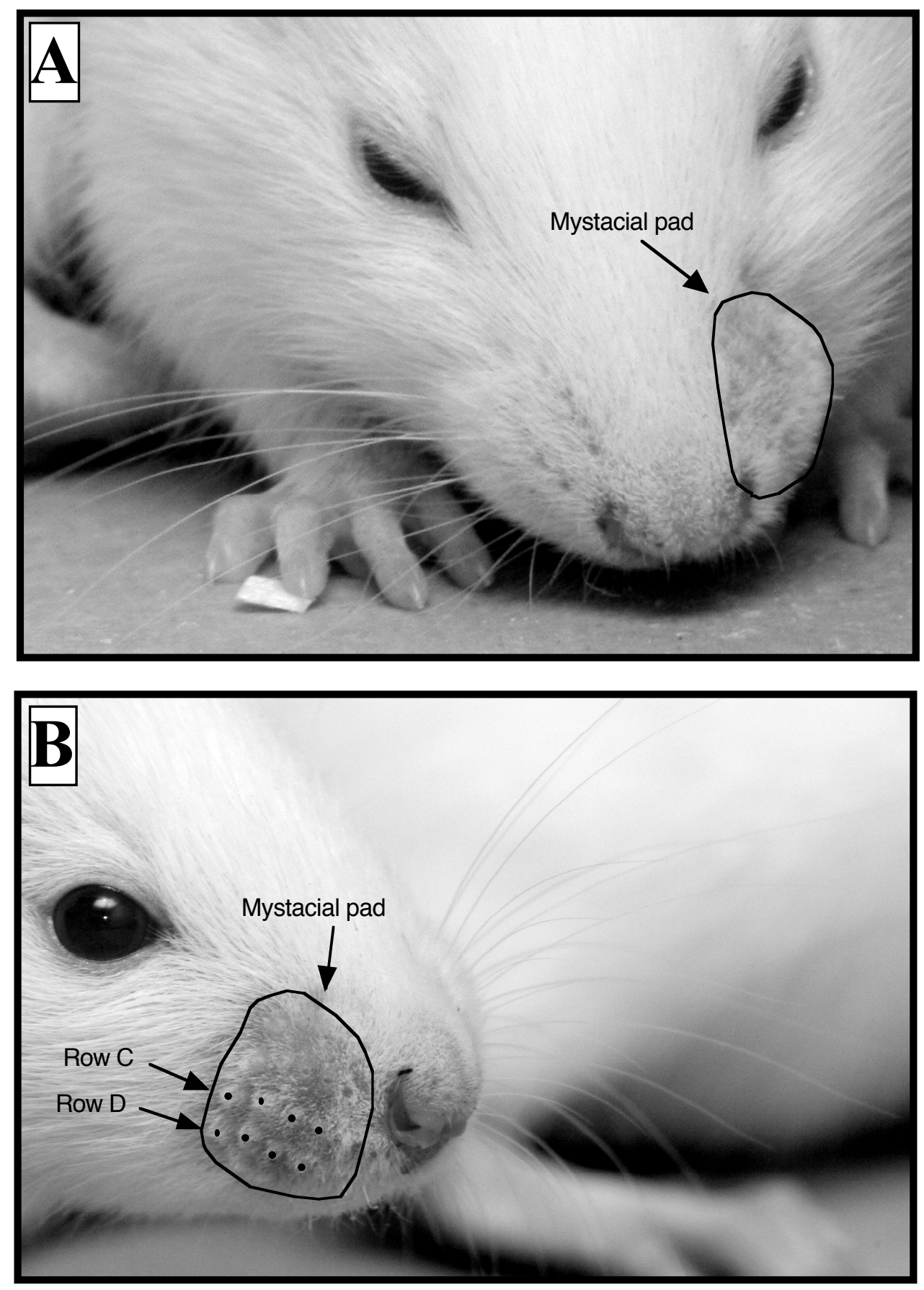

Figure 2.1 Trimmed unilateral mystacial pads in juvenile rats.

A) Frontal view of a 3-week-old rat that had the left mystacial pad trimmed. The absence of whiskers can clearly be seen. B) A lateral view of a 3-week-old rat with the right mystacial pad trimmed. Notice the small white bumps in B marked with black dots, indicating the locations where row $\mathrm{C}$ and $\mathrm{D}$ whiskers would normally have been found. These white bumps/black dots now mark the whisker follicle base. 
forebrain weighed again. The forebrain was hemi-sected, the white matter of each hemisphere was removed and the remaining gray matter of each hemisphere was flattened between two Plexiglas plates compressed together with rubber washers fitted around rivet posts. The cortical hemispheres of the vibrissae trimmed and adult rats were flattened by adjusting hexagonal nuts fitted to fine-threaded machine bolts. The flattened hemispheres of all animals were then placed in paraformaldehyde and refrigerated overnight. Following one day in paraformaldehyde, flattened hemispheres were sectioned in the sagittal plane at $120 \mu \mathrm{m}$ on a vibratome and placed in separate test tubes, for each hemisphere, containing $0.01 \mathrm{M}$ potassium phosphate buffer solution (KPBS, $\mathrm{pH}$ 7.4, $21^{\circ} \mathrm{C}$ ). Because the flattening of the hemispheres was more uniform across sections in the seven month-old rats and the vibrissae trimmed rats, it appeared that the tissues of these animals were less compressed than those of non-trimmed juvenile rats. Therefore, we were unable to compare the PMBSF measurements between the two age groups or between trimmed and non-trimmed juvenile groups.

\subsubsection{Tissue staining}

Sections were washed in KPBS $(3 \times 10 \mathrm{~min}$.) at room temperature. The tissue was stained with cytochrome oxidase using a method modified from Wong-Riley and Welt (1980) First, the tissue sections were rinsed in KPBS, the test tubes with the tissue sections were filled with a solution of $30 \mathrm{ml}$ 0.01M KPBS, $22.5 \mathrm{mg}$ diamenobenzidine (DAB, Sigma), $12 \mathrm{mg}$ cytochrome oxidase (Sigma), and $1.5 \mathrm{mg}$ sucrose (Fisher Chemicals) and the tubes were placed in a $37^{\circ} \mathrm{C}$ water bath for 2 hours or until barrels were clearly discernible from interbarrel septal regions. Finally, sections were rinsed 
$3 \times 10 \mathrm{~min}$. in $0.01 \mathrm{M} \mathrm{KPBS}$, mounted on gelatin coated slides, and coverslipped using permount/xylene.

\subsubsection{Quantitative PMBSF morphometric analysis}

Tissue sections were viewed with a light microscope using a $2 \times$ objective. A blue gelatin filter (Kodak, Wratten gelatin filter \#47A) was used to improve visualization of the barrel field. Images were captured with a digital camera and imported to Photoshop 7.0 on a Macintosh G5 computer where digitized sections containing the PMBSF were aligned, stacked, and reconstructed. The PMBSF consists of 27 well-demarcated barrels consisting of five rows (Row A to E) and four Straddler barrels located in the posterior portion of PMBSF $(\alpha, \beta, \gamma, \delta)$. In addition to the PMBSF, other barrel subfields were present and included the anterolateral barrel subfield (ALBSF) representing the upper lip and sinus hairs of the snout, lower lip barrel subfield (LLBSF), forepaw barrel subfield (FBS), hindpaw barrel subfield (HPBSF), and a nebulously-stained trunk subfield (TKS).

The present study focused exclusively on measurements of the PMBSF area, the barrel area of PMBSF, and the septal area lying between barrels. These areas are illustrated in the line drawings of the non-trimmed groups in Fig 2.2. The total PMBSF area was drawn by placing a boundary line around the reconstructed PMBSF as shown in Fig 2.2A'. The total area of individual barrels is shown in Fig 2.2B', while the septal area is shown by the blackened region in Fig $2.2 \mathrm{C}^{\prime}$. Digital reconstructions of PMBSF area, PMBSF barrel area, and PMBSF septal area were measured using Image J (NIH, Wayne Rashband). When two hemispheres from the same animal were available, both 


\section{Figure 2.2 The juvenile barrel cortex emphasizing PMBSF barrel pattern.}

Barrel field pattern and barrel area measurements in 6-week-old (juvenile) rats across chow-fed (CF), pair-fed (PF) and ethanol-treated (EtOH) groups. A) Digital image of the barrel field in layer IV in a CF rat. $\mathbf{A}^{\prime}$ ) Barrel field reconstruction delineating the major subfields of the rodent barrel cortex (posterior medial barrel subfield, PMBSF; anterolateral barrel subfield, ALBSF; lower lip barrel subfield, LLBSF; forepaw barrel subfield, FBS; hindpaw barrel subfield, HBS; trunk subfield, TKS). Line surrounding the 27 barrels of PMBSF indicates the measured area defining the total PMBSF area. Nomenclature within PMBSF barrels indicates the orientation of the anterior-posterior running barrel rows (A through $\mathrm{E}$ ) and the locations of the lateral-medial running arcs (barrels 1 through 5). The most posterior lateral to medial running barrels constitute the Straddler barrels as they lie between, or straddle, the end of the barrel rows; the Straddler barrels are designated as $\alpha, \beta, \gamma, \delta$ barrels). B) Digital image of barrel cortex in PF rat. $\left.B^{\prime}\right)$ The shaded region within the PMBSF shows the area of each barrel and is used to define the barrel area of PMBSF. C) Digital image of barrel field in EtOH rat. C') The blackened inter-barrel region within the PMBSF denotes the septal area. Septal area was calculated by subtracting total barrel area from total PMBSF area. Note that the general barrel field pattern appears similar across the groups. Scale bar $=500 \mu \mathrm{m}$. 

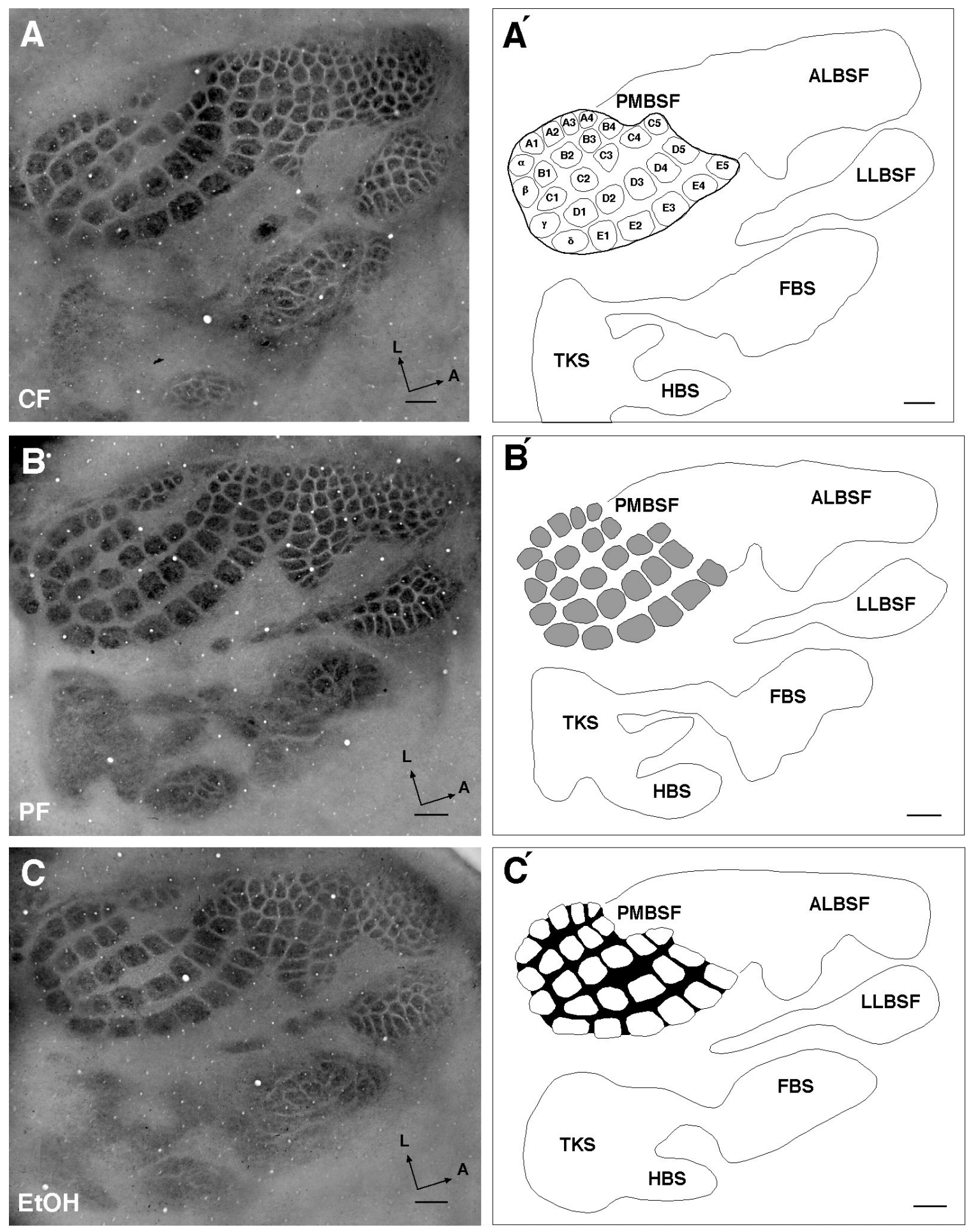
hemispheres were measured and averaged. In contrast, in vibrissae trimmed EtOH and CF rats, hemisphere measurements remained separate and were analyzed independently. Statview (version 5.1) or Data Desk (6.1) was used to perform all statistical analyses. All the groups were compared using a one-way ANOVA and the level of significance $(\alpha)$ was set at 0.05 . When ANOVA revealed a statistically significant effect, pair-wise comparisons were carried out using Tukey-Kramer's post-hoc test. To examine possible differential regional effects of $\mathrm{PAE}$, data from the $\mathrm{EtOH}$ group was calculated as a percent difference from respective $\mathrm{CF}$ and $\mathrm{PF}$ data. The forebrain/total PMBSF ratio was determined by dividing the forebrain weight by the total PMBSF area of each animal. This ratio was used to determine a possible asymmetric effect of PAE on forebrain and total PMBSF area. Also, the Pearson-Product-Moment correlation was used to assess the degree of relationship between the forebrain size and PMBSF area.

\subsection{RESULTS}

\subsubsection{Maternal blood alcohol levels}

Blood samples taken from EtOH dams had an average peak blood alcohol level (BAL) of $285.8 \pm 12.8 \mathrm{mg} / \mathrm{dl}$ (range; $210.4 \mathrm{mg} / \mathrm{dl}$ to $327.6 \mathrm{mg} / \mathrm{dl}$ ) on GD13 and $329.1 \pm$ $21.0 \mathrm{mg} / \mathrm{dl}$ (range; $207.4 \mathrm{mg} / \mathrm{dl}$ to $478.6 \mathrm{mg} / \mathrm{dl}$ ) on GD20. EtOH dams, whose pups had their vibrissae trimmed, had peak BALs of $243 \pm 2.4 \mathrm{mg} / \mathrm{dl}$ (range $=236 \mathrm{mg} / \mathrm{dl}$ to 267 $\mathrm{mg} / \mathrm{dl}$ ) on GD10 and $382 \pm 10 \mathrm{mg} / \mathrm{dl}$ (range $=343 \mathrm{mg} / \mathrm{dl}$ to $463 \mathrm{mg} / \mathrm{dl}$ ) on GD17. 


\subsubsection{Effect of PAE on PMBSF in juveniles}

\subsubsection{The pattern of PMBSF in juveniles}

Representatives of the PMBSF pattern for juvenile rats (non-trimmed) in the CF, $\mathrm{PF}$, and EtOH groups are illustrated in Fig 2.2. Note that the five-row barrel arrangement and the presence of the posteriorly located Straddler barrels are seen in each group. The PMBSF consists of 27 barrels, and likely reflects a common plan of organization that is independent of treatment. A similar PMBSF barrel pattern for all groups was also observed in adult rats (data not shown). Thus, PAE does not appear to alter the overall PMBSF pattern in juvenile or adult rats.

\subsubsection{The area of PMBSF in juveniles}

Table 2.1 shows the mean \pm SEM of the total PMBSF areas for the four nontrimmed groups. A significant main effect was observed for total PMBSF area $[\mathrm{F}(3,80)=$ $6.357, \mathrm{p}=0.0006$ ] between all groups. Total averaged PMBSF area was significantly reduced in the EtOH group compared to the CF group and PF groups. No significant differences were observed between EtOH and XF groups or between $\mathrm{CF}$ and PF groups. When the data was calculated as a percent difference from CF, results showed that the PMBSF area in the EtOH group was $13.1 \pm 1.9 \%$ smaller than that of the rats in CF group and $10.2 \pm 1.9 \%$ smaller than the PF group. 
Table 2.1 PMBSF areas in juveniles.

\begin{tabular}{|c|c|c|c|c|}
\hline Treatment & $\mathrm{EtOH}(n=17)$ & $\mathrm{XF}(n=15)$ & $\mathrm{CF}(n=25)$ & $\mathrm{PF}(n=27)$ \\
\hline PMBSF area $\left(\mathrm{mm}^{2}\right)$ & $4.69^{\mathrm{a}, \mathrm{b}}(0.10)$ & $4.74^{\mathrm{c}, \mathrm{d}}(0.15)$ & $5.40(0.10)$ & $5.22(0.16)$ \\
\hline PMBSF barrel area $\left(\mathrm{mm}^{2}\right)$ & $3.27^{\mathrm{a}, \mathrm{b}}(0.07)$ & $3.29^{\mathrm{c}, \mathrm{d}}(0.09)$ & $3.81(0.07)$ & $3.74(0.11)$ \\
\hline PMBSF septal area $\left(\mathrm{mm}^{2}\right)$ & $1.43 \quad(0.04)$ & $1.45 \quad(0.06)$ & $1.59(0.04)$ & $1.48(0.05)$ \\
\hline
\end{tabular}

Data are presented as mean $\pm \mathrm{SEM}$. A significant difference between groups indicates a $\mathrm{P}$-value of $(\mathrm{P}<0.05)$. EtOH versus $\mathrm{CF}={ }^{\mathrm{a}}, \mathrm{EtOH}$ versus $\mathrm{PF}={ }^{\mathrm{b}}, \mathrm{XF}$ versus $\mathrm{CF}={ }^{\mathrm{c}}, \mathrm{XF}$ versus $\mathrm{PF}={ }^{\mathrm{d}}$. 


\subsubsection{PMBSF barrel area of juveniles}

Analysis of the barrel area of PMBSF revealed a significant main effect $[\mathrm{F}(3,80)$

$=7.589, \mathrm{p}<0.0002]$ for all groups (Table 2.1). The area of the PMBSF barrels was significantly reduced in the EtOH group compared to non-alcohol controls. No significant differences were seen between EtOH and XF groups or between CF and PF groups. Data indicated that the total barrel area of PMBSF in the EtOH group was $14.3 \pm 1.9 \%$ smaller than the total barrel area of PMBSF in CF group and $12.5 \pm 2.0 \%$ smaller than the PF group.

\subsubsection{PMBSF septal area of juveniles}

The mean PMBSF septal area for each group is shown in Table 2.1. The septa regions were not significantly different $[F(3,80)=2.546, p=0.0618]$ between the groups.

\subsubsection{PAE produced intraregional asymmetries in PMBSF of juveniles}

Previously, PD10 EtOH pups were reported to have anterior-to-posterior asymmetries that indicated the posterior PMBSF barrels were less affected by PAE than were anterior barrels compared to respective barrels in the CF group (Margret et al. 2005). To determine whether this effect continued into juvenile and adult rats, we measured and compared the barrel area for each of the 27 barrels for PAE and non-PAE treated rats. Individual barrels for $\mathrm{EtOH}$ and $\mathrm{CF}$ groups were compared on a barrel-bybarrel basis and 74\% (20/27) of the barrels were significantly smaller in the EtOH group compared to the CF group (Fig 2.3). The barrels that were significantly different between these two groups are indicated with asterisks. No significant differences in barrel areas 


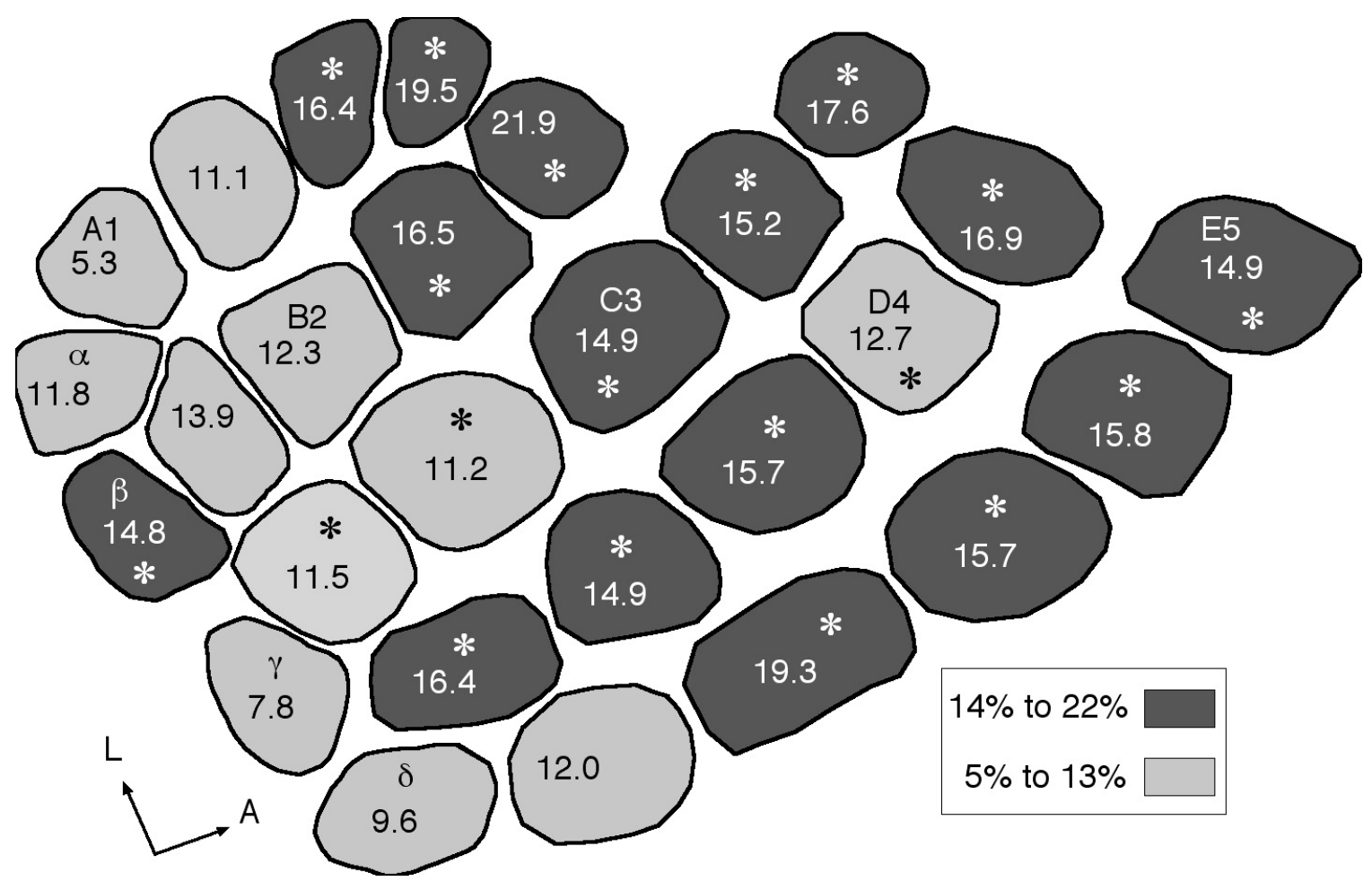

Figure 2.3 Barrel asymmetries within the PMBSF of PAE juvenile rats.

Comparison of individual barrel areas in PMBSF between EtOH and CF groups in juvenile rats at 6 weeks of age. Each barrel area in the EtOH group was calculated as a percent change from the respective barrel in the CF group. The percent reduction in the area of the barrels in the EtOH group ranged from 5.3\% to 21.9\% smaller in EtOH group compared to the CF group. The midpoint in this range was used to partition the PMBSF between less reduced barrels in posterior barrels (lightly shaded) of the PMBSF from more reduced anterior barrels (dark shading). Each barrel is annotated with a number indicating the percent reduction in barrel area in the $\mathrm{EtOH}$ group compared to the $\mathrm{CF}$ group. Asterisks denote barrels that were significantly reduced in the EtOH group compared to the CF group $(\mathrm{P} \leq 0.05)$. 
between EtOH and XF or between PF and CF groups were identified. The areas of barrels of rats in the EtOH group were calculated as a percent difference from a respective barrel in the CF group and categorized as follows: (i) $5 \%$ to $13 \%$ reduced, (ii) $14 \%$ to $22 \%$ reduced. The areas of the anterior barrels of rats in the EtOH group were more reduced compared to anterior barrels of rats in CF group (Fig 2.3) although this difference was not significant (data not shown). In addition, the majority of the areas of posterior barrels of rats in the EtOH group were modestly smaller compared to those in the CF group.

\subsection{PMBSF barrel rows in juveniles}

We next compared barrel rows (A to E) and arcs (1 to 5 and Straddlers) between groups. Calculation of the row data in the EtOH group, as a percent change from $\mathrm{CF}$, showed little difference in percent reductions between lateral (A1 to A4) and medial (E1 to E5) rows $[12.1 \%, 15.8 \%, 13.8 \%, 15.3 \%$, and $15.7 \%$, respectively] (Fig 2.4C). A significant effect did occur between EtOH and CF groups in all rows. Post-hoc analysis (Tukey- Kramer's) revealed that individually, rows B to E were significantly reduced in the $\mathrm{EtOH}$ group compared to the $\mathrm{CF}$ group (Fig 2.4B). However, rows $\mathrm{C}$ and A were not significantly different between EtOH and PF groups.

\subsection{PMBSF barrel arcs in juveniles}

The percent change of arcs in EtOH compared to the CF group (combination of lateral to medial running barrels in a column) showed less reduction in posterior arcs compared to anterior arcs $[10.8 \%, 12.2 \%, 14.3 \%, 15.8 \%, 16.2 \%$ and $16.3 \%$, respectively $]$ (Fig 2.4D). The differential effect in EtOH group posterior barrels and arcs in relation to 

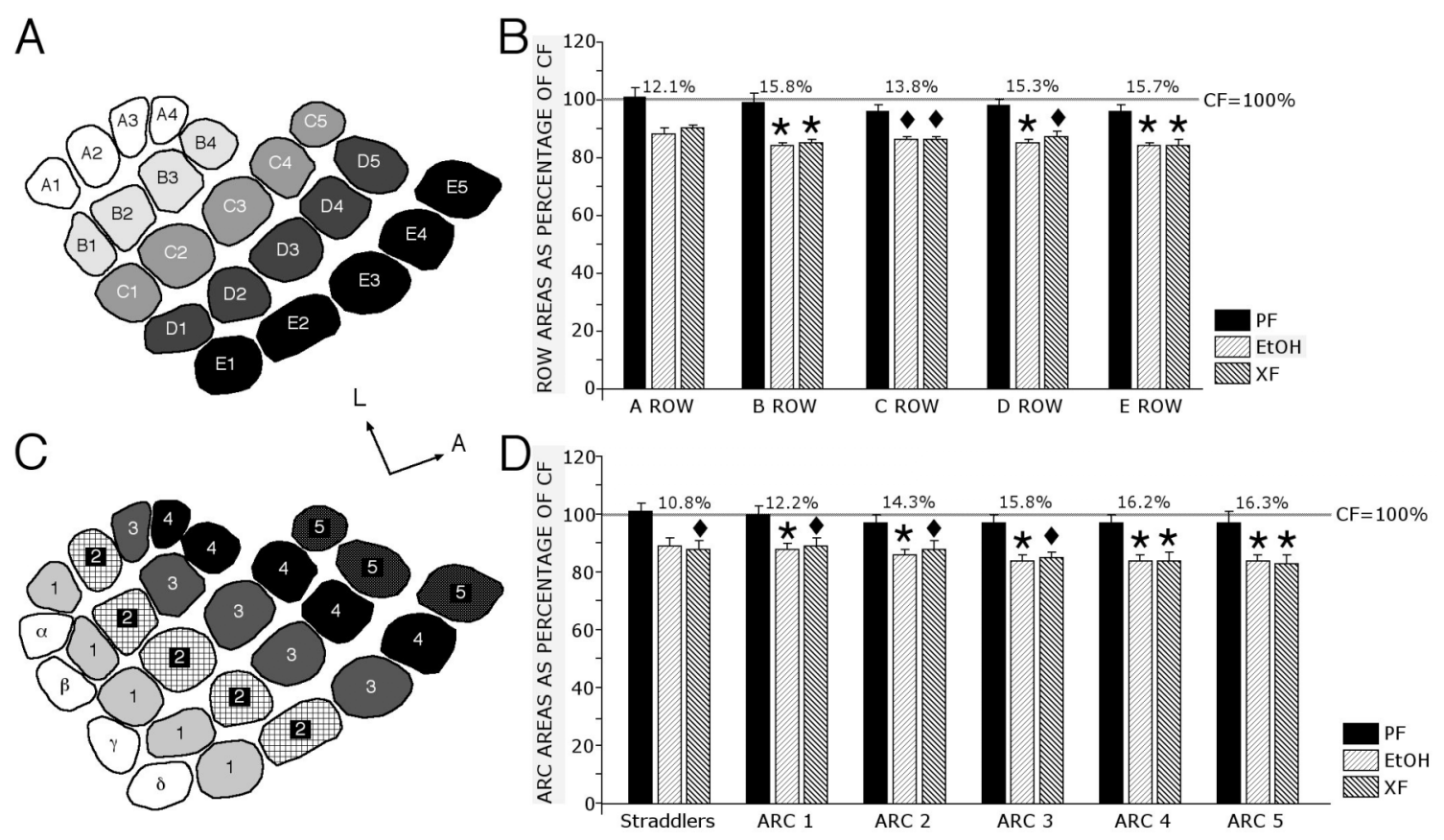

Figure 2.4 Juvenile intraregional arc and row vulnerability within PMBSF.

PMBSF row and arc areas in $\mathrm{EtOH}, \mathrm{XF}$, and $\mathrm{PF}$ groups compared to $\mathrm{CF}$ groups at 6weeks of age. Schematic diagrams showing A) barrel rows, or B) arcs and Straddler barrels. C) Graph showing row areas for EtOH, XF, and PF groups calculated as a percentage change of $\mathrm{CF}$ group are shown in $\mathrm{C}$. In this histogram, there are significant area reductions between $\mathrm{EtOH}$ and $\mathrm{CF}$ rats for rows $\mathrm{B}-\mathrm{E}$, but no significant row differences between $\mathrm{CF}$ and $\mathrm{PF}$ or between EtOH and XF. Percent reduction in row area between EtOH and CF is indicated above the bars for each of the rows. D) Graph showing percent arc and Straddler areas for $\mathrm{EtOH}, \mathrm{XF}$, and $\mathrm{PF}$ rats calculated as a percentage change of CF rats. The Straddler arc showed no significant difference between $\mathrm{EtOH}$ and $\mathrm{CF}$, while arcs 1-5 were significantly reduced. No significant differences occurred between $\mathrm{CF}$ and PF or between EtOH and XF arc areas. Percent reduction in arc area between $\mathrm{EtOH}$ and $\mathrm{CF}$ is indicated above the bars of each arc/Straddler. Note the gradual asymmetry moving from posterior to anterior arcs, which is not seen between rows. Gray lines in $\mathbf{C}$ and $\mathbf{D}$ indicate percent change from $\mathrm{CF}$ values at $100 \%$. Diamonds $(\checkmark)$ indicate significant differences between EtOH and CF or between XF and CF. Asterisks (*) indicate significant differences between EtOH and both $\mathrm{CF}$ and $\mathrm{PF}$ groups or between $\mathrm{XF}$ and both $\mathrm{CF}$ and $\mathrm{PF}$ groups. 
anterior barrels and arcs is heretofore referred to as an "asymmetry" in the PMBSF.

The data from arc measurements in the EtOH group suggested a trend towards an asymmetrical pattern between posterior and anterior barrels. This asymmetry indicated that arcs positioned anteriorly were more reduced than posterior arcs. All arc comparisons resulted in a significant reduction between the EtOH group and the $\mathrm{CF}$ or PF groups. Post-hoc analysis (Tukey- Kramer's) revealed that all arcs were significantly reduced in the $\mathrm{EtOH}$ group compared to the $\mathrm{CF}$ and $\mathrm{PF}$ groups except the Straddler arc as shown in Fig 2.4D.

\subsubsection{Effect of PAE on body weight in juveniles}

There was a significant reduction in body weight between the groups $[\mathrm{F}(3,80)=$ $4.348, \mathrm{p}=0.0069]$. The body weight was significantly reduced in the EtOH group compared to PF, but no significant differences occurred between the other groups (Table 2.2). The mean body weight of the EtOH group was divided by the mean body weight of the PF group. The body weight of the EtOH group was reduced $18.6 \pm 3.1 \%$ compared to the PF group. No significant effect occurred between PF and CF groups.

\subsubsection{Effect of PAE on brain weight in juveniles}

PAE produced a significant main effect on whole brain $[\mathrm{F}(3,80)=19.804, \mathrm{p}<$ 0.0001] and forebrain $[F(3,80)=17.094, p<0.0001]$ weights. Whole brain and forebrain weights were significantly lower in the EtOH group compared to controls (Table 2.2). No significant differences in brain weight were found between $\mathrm{EtOH}$ and $\mathrm{XF}$ rats or between 
Table 2.2 The effect of PAE on body, brain weights, and forebrain/PMBSF ratio in juveniles.

\begin{tabular}{lcccc}
\hline Treatment & $\mathrm{EtOH}(n=17)$ & $\mathrm{XF}(n=15)$ & $\mathrm{CF}(n=25)$ & $\mathrm{PF}(n=27)$ \\
\hline Body weight $(\mathrm{g})$ & $156.12^{\mathrm{b}}(6)$ & $179.67(11)$ & $180.75(5)$ & $191.86(7)$ \\
Whole brain weight $(\mathrm{g})$ & $1.48^{\mathrm{a}, \mathrm{b}}(0.03)$ & $1.47^{\mathrm{c}, \mathrm{d}}(0.03)$ & $1.61(0.01)$ & $1.63(0.02)$ \\
Forebrain weight $(\mathrm{g})$ & $1.07^{\mathrm{a}, \mathrm{b}}(0.02)$ & $1.06^{\mathrm{c}, \mathrm{d}}(0.02)$ & $1.15(0.01)$ & $1.18(0.01)$ \\
Forebrain/PMBSF area ratio & $0.23(0.01)$ & $0.23(0.01)$ & $0.21(0.01)$ & $0.23(0.01)$ \\
\hline
\end{tabular}

Data are presented as mean \pm SEM. A significant difference between groups indicates a $\mathrm{P}$-value of $(\mathrm{P}<0.05)$. EtOH versus $\mathrm{CF}={ }^{\mathrm{a}}, \mathrm{EtOH}$ versus $\mathrm{PF}={ }^{\mathrm{b}}, \mathrm{XF}$ versus $\mathrm{CF}={ }^{\mathrm{c}}, \mathrm{XF}$ versus $P F={ }^{d}$. 
$\mathrm{CF}$ and PF rats. EtOH whole brain and forebrain weights were reduced by $8.3 \pm 1.6 \%$ and $6.9 \pm 1.5 \%$, respectively compared to CF rats, while the EtOH group was reduced $9.5 \pm$ $1.6 \%$ and $9.1 \pm 1.5 \%$, respectively compared to the PF group.

\subsubsection{Forebrain/PMBSF ratio in juveniles}

PAE significantly reduced both forebrain weight and PMBSF area. We used forebrain/PMBSF ratio to determine whether alcohol played a greater role on either brain weight or PMBSF area, or whether both were equally affected. PAE had no significant effect on the ratio across groups, suggesting that both brain weight and PMBSF area were equally affected (Table 2.2).

\subsubsection{Correlation between forebrain weight and total PMBSF area in juveniles}

A regression analysis indicated (Fig 2.5) that forebrain weight was not highly correlated to the area of PMBSF in any of the groups (EtOH: $r=0.23, \mathrm{XF}: \mathrm{r}=-0.29, \mathrm{PF}$ : $r=-0.22, C F: r=0.40)$

\subsubsection{Effect of PAE on PMBSF in adults}

\subsubsection{PMBSF barrel pattern in adults}

No obvious differences were observed in the positions of the PMBSF or individual barrels within the PMBSF between EtOH, XF, and PF treatment groups, and CF control group (data not shown). There were no missing barrels within the PMBSF in any of the reconstructed brain sections from any group. Also, the positions of the 

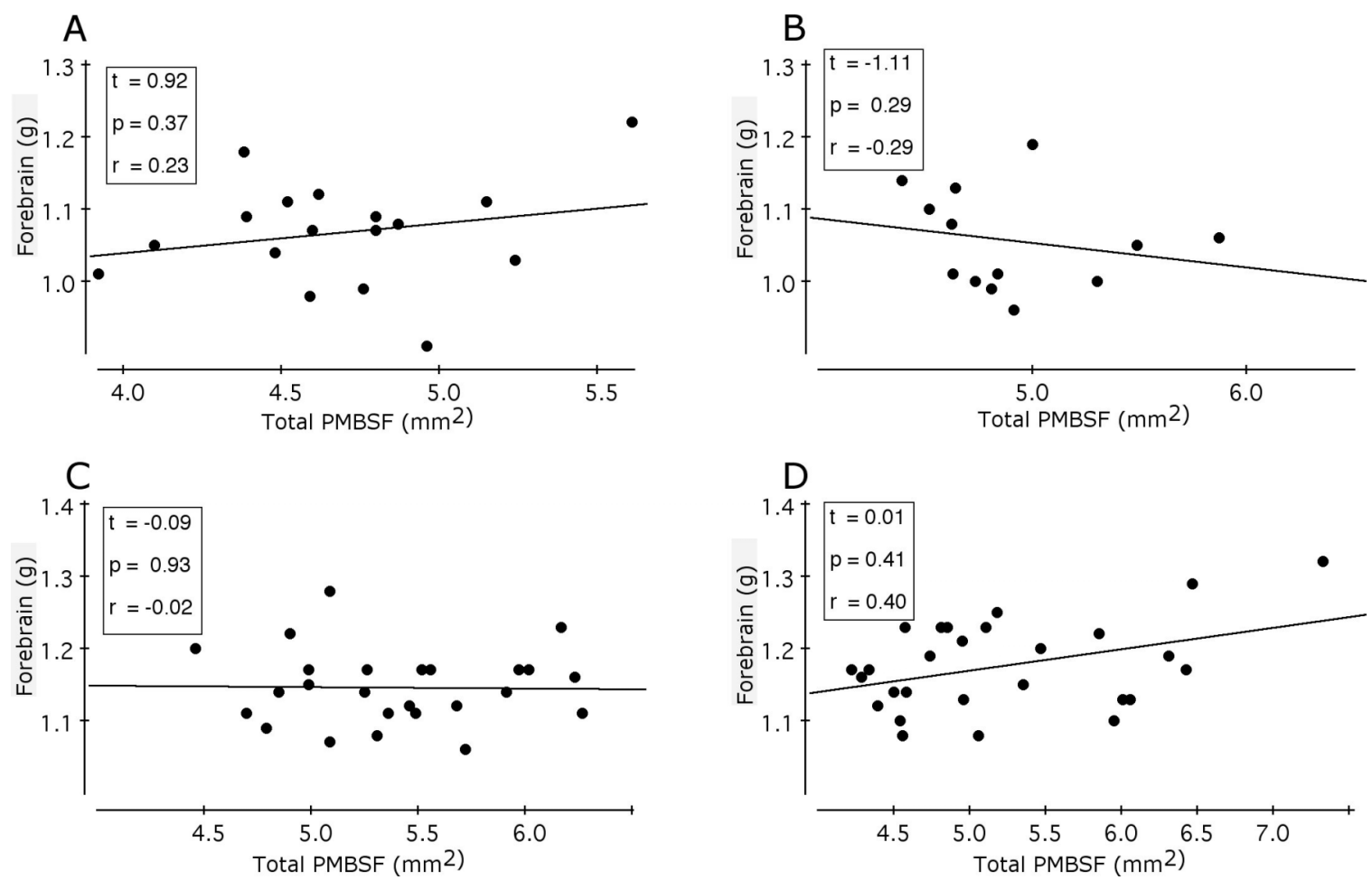

\section{Figure 2.5 Correlation between forebrain weight and total PMBSF area in juveniles.}

Pearson Product-Moment Correlation and linear regression analyses [t-ratio] of forebrain weight and total PMBSF area for each group in 6-week-old rats. A) Forebrain weight and total PMBSF area correlation and regression analyses for EtOH group. Inset provides tratio, probability (p), and correlation coefficient (r) values. B) Correlation for XF group, C) PF group, and D) CF group. 
individual barrels within the PMBSF were similar between groups.

\subsubsection{The PMBSF area in adults}

The adult PMBSF area was significantly smaller in the EtOH group compared to the PF and CF groups $[F(2,22)=5.017, p=0.016]$ (Table 2.3). There were no significant differences in PMBSF area between the CF and PF groups. Data indicated that the PMBSF area in the EtOH group was $8.9 \pm 2.0 \%$ reduced compared to CF group and $9.6 \pm$ $1.9 \%$ reduced compared to the PF group.

\subsubsection{The PMBSF barrel area in adults}

The PMBSF barrel area in the EtOH group was significantly smaller compared to $\mathrm{PF}$ and $\mathrm{CF}$ rats $[\mathrm{F}(2,22)=6.433, \mathrm{p}=0.0063]$; these data are illustrated in Table 2.3. No significant differences were found between non-alcohol groups. PAE reduced mean total barrel area by $12.7 \pm 1.7 \%$ in EtOH rats compared to rats in the CF group while a reduction of $9.3 \pm 1.8 \%$ occurred between EtOH and PF groups.

\subsubsection{PMBSF septal area in adults}

No significant effect was observed for the total septal area $[F(2,22)=2.741, p=$ 0.0865] as shown in Table 2.3. 
Table 2.3 PMBSF areas in adults.

\begin{tabular}{|c|c|c|c|}
\hline Treatment & $\mathrm{EtOH}(n=9)$ & $\mathrm{CF}(n=8)$ & $\mathrm{PF}(n=8)$ \\
\hline Total PMBSF area $\left(\mathrm{mm}^{2}\right)$ & $4.30^{\mathrm{a}, \mathrm{b}}(0.09)$ & $4.73(0.12)$ & $4.76(0.13)$ \\
\hline Total barrel area of the PMBSF $\left(\mathrm{mm}^{2}\right)$ & $2.98^{\mathrm{a}, \mathrm{b}}(0.06)$ & $3.41(0.12)$ & $3.28(0.10)$ \\
\hline Total PMBSF septa $\left(\mathrm{mm}^{2}\right)$ & $1.33 \quad(0.06)$ & $1.32(0.04)$ & $1.48(0.06)$ \\
\hline
\end{tabular}

Data are presented as mean $\pm \mathrm{SEM}$. A significant difference between groups indicates a $\mathrm{P}$-value of $(\mathrm{P}<0.05)$. EtOH versus $\mathrm{CF}={ }^{\mathrm{a}}$, EtOH versus $\mathrm{PF}={ }^{\mathrm{b}}$ 


\subsubsection{PMBSF intraregional vulnerability to $P A E$ in adults}

Similar to results from the experiment on juvenile rats, the total barrel area of PMBSF was significantly smaller in adult rats in the EtOH group compared to those in the $\mathrm{CF}$ and $\mathrm{PF}$ groups. When the areas of individual barrels in the $\mathrm{EtOH}$ group were compared to the areas of identical barrels of juvenile rats in the EtOH group, $63 \%(17 / 27)$ of the barrels were smaller in juvenile rats compared to $48 \%(13 / 27)$ in adult rats. Asterisks in Fig 2.6 indicate significantly smaller barrels. No significant differences in individual PMBSF barrel areas occurred between the PF and CF groups. We next examined the barrels of rats in the $\mathrm{EtOH}$ group as a percent change from the $\mathrm{CF}$ group and categorized the results as shown in Fig 2.6. The juvenile EtOH group asymmetry persisted into adults as the reduction in the area of anterior barrels was greater than the reduction in posterior barrels.

\subsection{PMBSF barrel rows in adults}

Examination of rows of barrels revealed little change in percent reduction moving from lateral to medial rows and no asymmetry pattern was identified (A row $=12.2 \%, \mathrm{~B}$ row $=14.4 \%, \mathrm{C}$ row $=11.9 \%, \mathrm{D}$ row $=12.1 \%$, E row $15.6 \%)$. Tukey-Kramer's post-hoc analysis revealed that all rows of barrels in the EtOH group were significantly smaller than those in PF or CF groups (Fig 2.7B). 


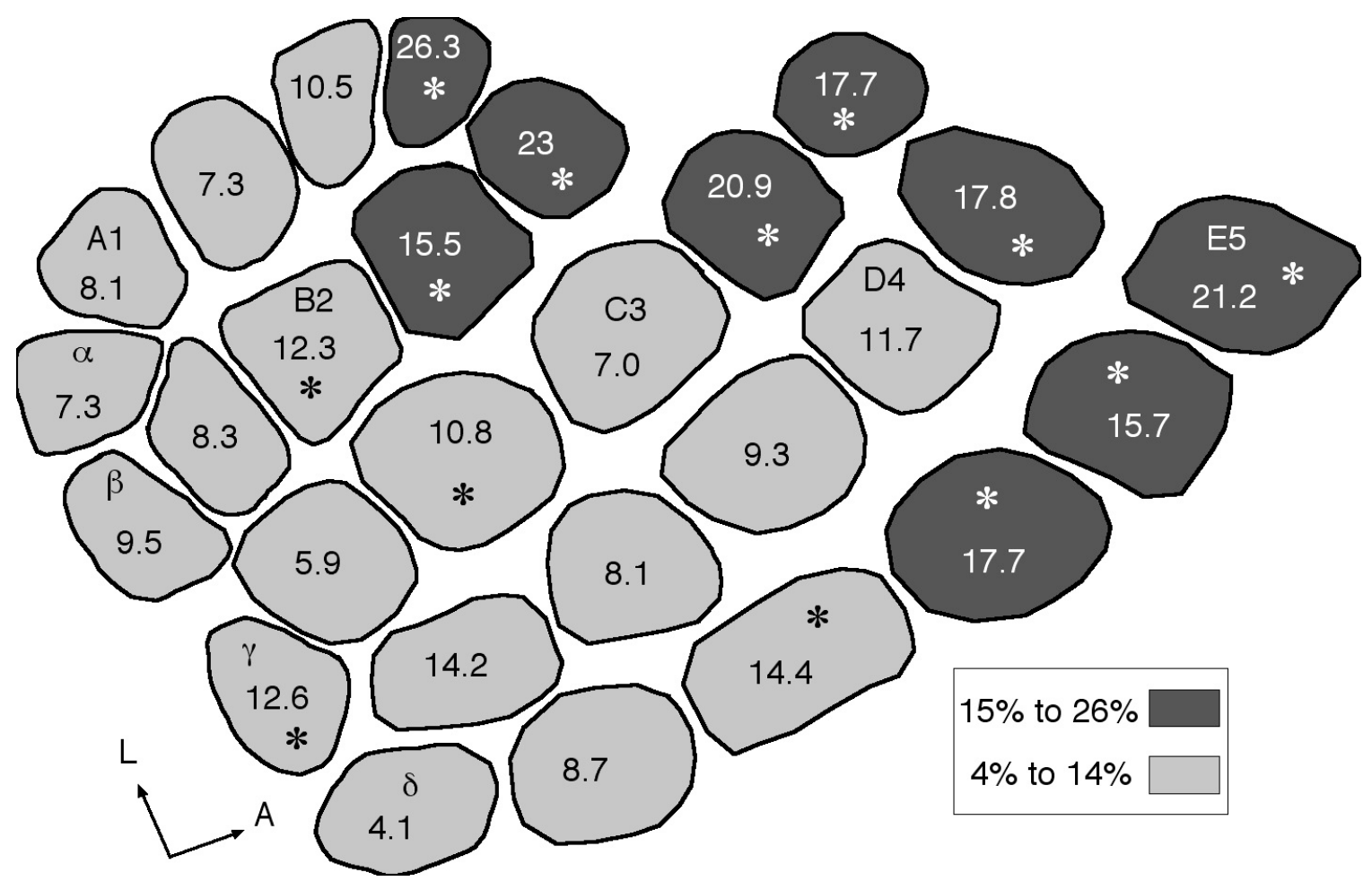

Figure 2.6 Barrel asymmetries within the PMBSF of PAE adults.

Comparison of individual barrel areas in PMBSF between EtOH and CF groups in adult rats at 7 months of age. Each barrel area in the EtOH group was calculated as a percent change from the respective barrel in the $\mathrm{CF}$ group. The percent reduction in the area of the barrels in the EtOH group ranged from $4.1 \%$ to $26.3 \%$ smaller in EtOH group compared to the CF group. The midpoint in this range was used to partition the PMBSF between less reduced barrels in posterior positions (lightly shaded) of the PMBSF to more reduced barrels in anterior regions (dark shading). Each barrel is annotated with a number indicating the percent reduction in barrel area in the EtOH group compared to the $\mathrm{CF}$ group. Asterisks denote barrels that were significantly reduced in the EtOH group compared to the $\mathrm{CF}$ group $(\mathrm{P} \leq 0.05)$. 


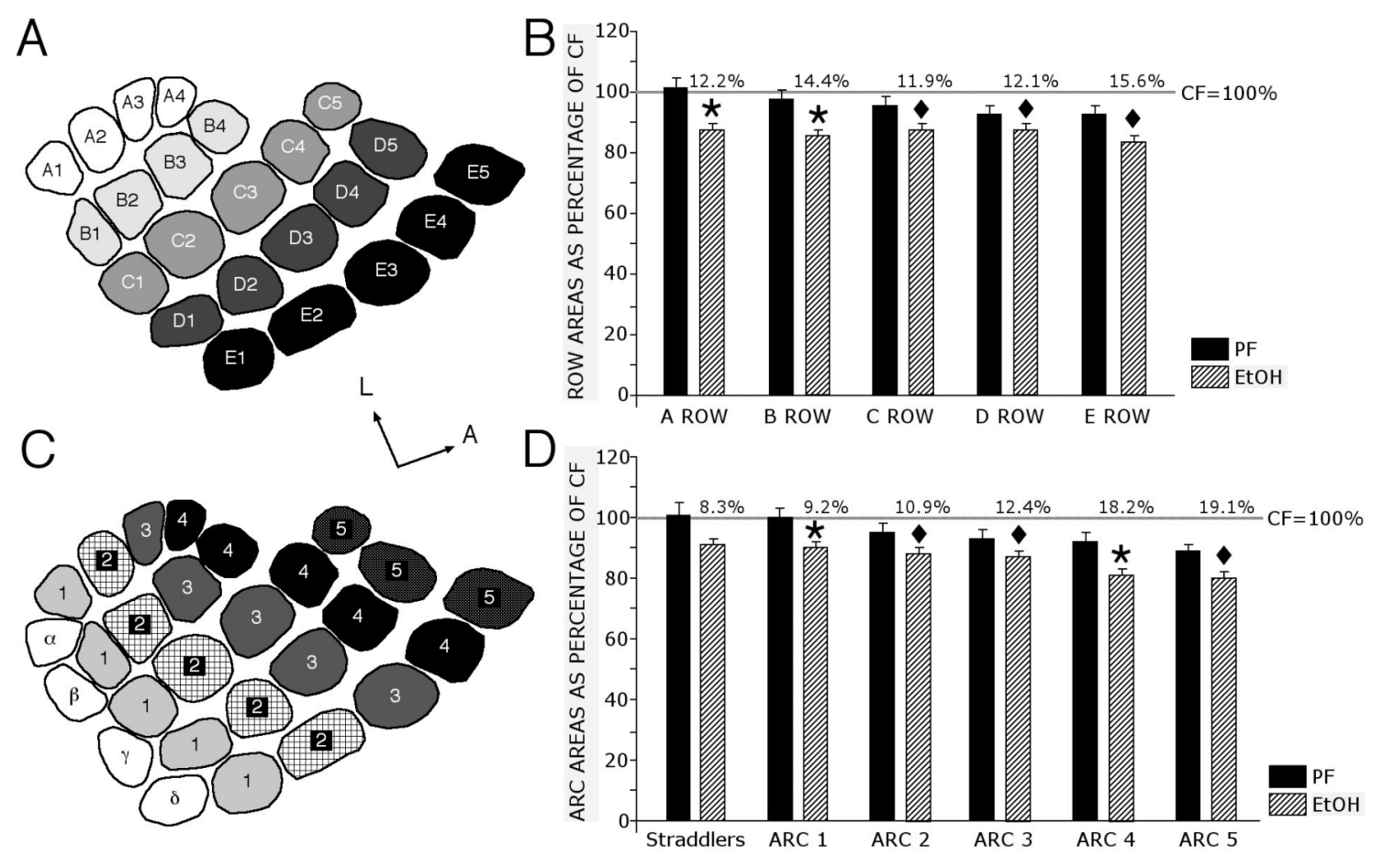

Figure 2.7 Intraregional PMBSF arc and row vulnerability within PMBSF of adults.

PMBSF row and arc areas in EtOH and PF rats were calculated as a percent change from CF rats at 7-months of age. Schematic diagrams showing A) barrel rows and B) arcs and Straddler barrels. C) Graph showing average percent row area for EtOH and PF rats calculated as a percentage change of CF rats. Significant reductions in row area were observed between EtOH and non-alcohol rats. No significant row differences occurred between $\mathrm{CF}$ and $\mathrm{PF}$ groups. Percent reduction in row area between $\mathrm{EtOH}$ and $\mathrm{CF}$ is indicated above the bars of each row. D) Graph showing averaged percent arc and Straddler areas for EtOH and PF rats calculated as a percentage change of CF rats. The Straddler arc showed no significant difference between EtOH and CF, while arcs 1-5 were significantly reduced from CF group. No significant differences occurred between $\mathrm{CF}$ and $\mathrm{PF}$ arc areas. Percent reduction in arc area between EtOH and CF is indicated above the bars of each arc. Gray lines in $\mathbf{C}$ and $\mathbf{D}$ indicate calculated $\mathrm{CF}$ values at $100 \%$. Diamond $(\diamond)$ indicates significant difference between EtOH and CF. Asterisk $\left(^{*}\right)$ indicates significant difference between $\mathrm{EtOH}$ and both non-alcohol groups. 


\subsection{PMBSF barrel arcs in adults}

In sharp contrast to barrel rows, asymmetries were observed between posterior and anterior arcs in the percent reduction between arcs in the EtOH group and those of the CF group (Straddlers 8.3\%, Arc 1 9.2\%, Arc 2 10.9\%, Arc 3 12.4\%, Arc 4 18.2\%, Arc $5=19.1 \%$ ). Tukey-Kramer's post-hoc analysis revealed that while all arcs in the EtOH group (except the Straddler arc) were significantly smaller than the CF group (Fig 2.7D) only Arcs 1 and 4 were significantly reduced in the EtOH group compared to the PF group.

\subsubsection{Effect of PAE on brain and body weights in adults}

No significant effect was observed in body or brain weights between any groups. The mean \pm SEM for each group is presented in Table 2.4.

\subsection{THE EFFECT OF PAE AND VIBRISSAE TRIMMING ON PMBSF IN JUVENILES}

Because PAE seemingly reduced the areas of the anterior barrels in juvenile rats more than the area of posterior barrels, we hypothesized that the posterior to anterior asymmetry identified in juveniles following PAE may be due to differential sensory experience in that the posterior whiskers may be stimulated to a greater extent than anterior whiskers during PD5 to PD42. 
Table 2.4 The effect of PAE on body and brain weights in adults.

\begin{tabular}{lccc}
\hline Treatment & EtOH $(n=9)$ & CF $(n=8)$ & PF $(n=8)$ \\
\hline Body weight $(\mathrm{g})$ & $383.83(40)$ & $400.31(40)$ & $405.94(37)$ \\
Whole brain weight $(\mathrm{g})$ & $1.80(0.05)$ & $1.89(0.04)$ & $1.93(0.05)$ \\
Forebrain weight $(\mathrm{g})$ & $1.27(0.04)$ & $1.31(0.03)$ & $1.35(0.03)$ \\
\hline
\end{tabular}

Data are presented as mean \pm SEM. No significant differences occurred between the groups. 


\subsubsection{Pattern of PMBSF in vibrissae trimmed juveniles}

Whisker trimming did not appear to alter barrel field pattern in any hemisphere from either treatment group. Inspection of the PMBSF showed no incidence of missing barrels, unusually displaced barrels or any gross deviation in overall shape of the PMBSF in any group (as shown in Fig 2.8).

\subsubsection{The area of the PMBSF in trimmed EtOH and CF juveniles}

The only significant reduction in the area of the PMBSF in trimmed EtOH or CF groups occurred in the EtOH-trim group which was significantly reduced compared to the $\mathrm{CF}$-intact group $[\mathrm{F}(3,50)=3.8, \mathrm{p}=0.0349]$. However, no significant differences in PMBSF area occurred between any other groups. Table 2.5 shows the mean \pm SEM of the PMBSF areas of each group

\subsubsection{PMBSF barrel area in trimmed EtOH and CF juveniles}

No significant differences occurred between the PMBSF barrel areas within or between the groups (EtOH-trim, EtOH-intact, $\mathrm{CF}$-trim, or CF-intact) $[\mathrm{F}(3,50)=1.885$, $\mathrm{p}$ $=0.1447]$ as shown in Table 2.5.

\subsubsection{Septal area in trimmed EtOH and $\mathrm{CF}$ juveniles}

The septal area of EtOH-trim group was significantly reduced compared to CFintact $[\mathrm{F}(2,38)=2.548, \mathrm{p}=0.0356]$ (Table 2.5). No significant differences occurred between any other groups. Data indicated that the EtOH-trim group was $10.5 \pm 2.5 \%$ reduced compared to the $\mathrm{CF}$-intact group. 

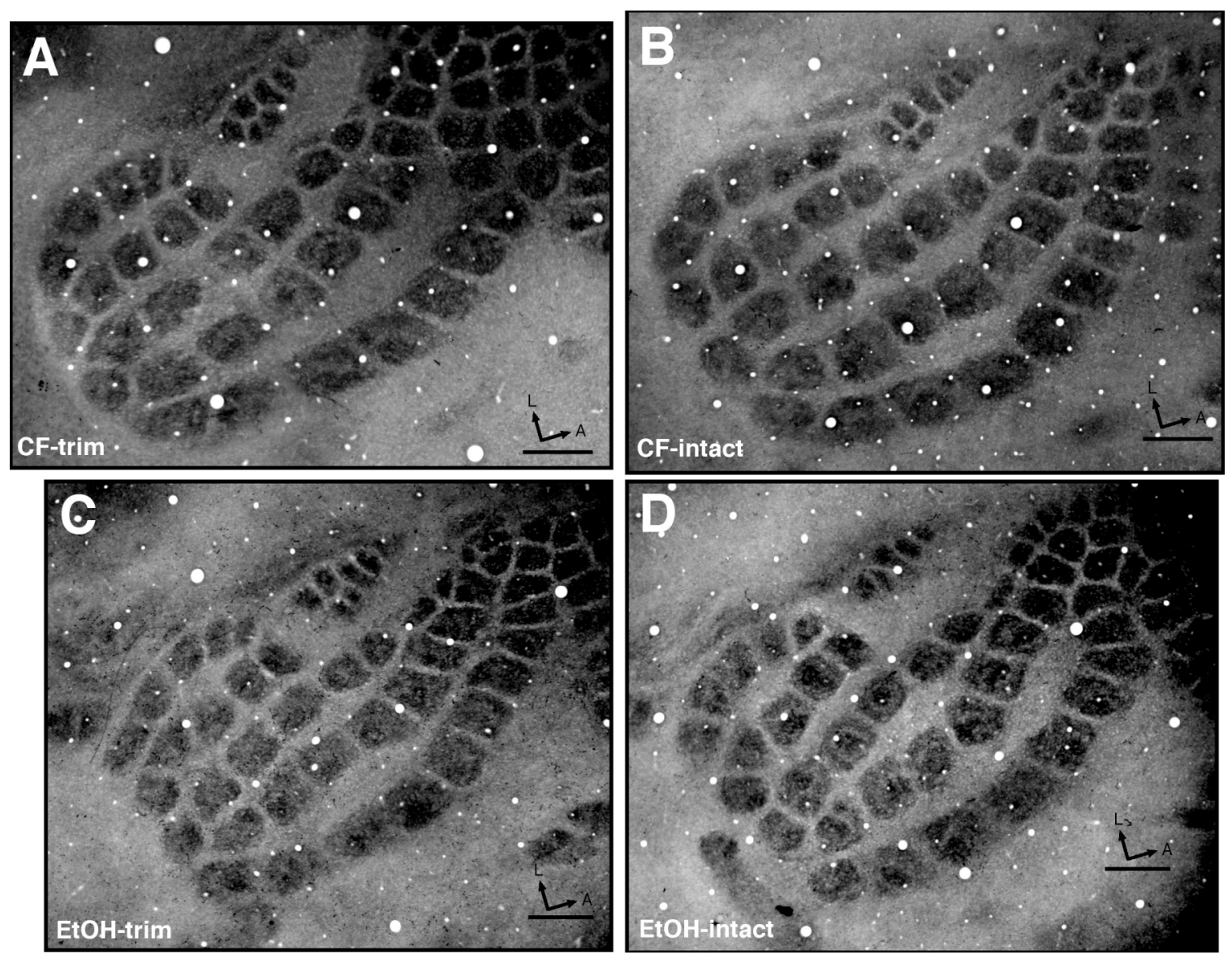

Figure 2.8 The PMBSF following unilateral vibrissae trimming in CF-trim, CFintact, EtOH-trim, and EtOH-intact groups.

The posterior medial barrel subfield (PMBSF) in juvenile rats across CF-trim, CF-intact, EtOH-trim, and EtOH-intact groups. A) Digital image of the contralateral PMBSF to unilateral vibrissae trimming of the mystacial pad (CF-trim). B) Digital image of the PMBSF from the ipsilateral hemisphere in relation to unilateral vibrissae trimming (CFintact). C) Digital image of the contralateral PMBSF to vibrissae trimming (EtOH-trim) D) Digital image of the ipsilateral PMBSF to vibrissae trimming (EtOH-intact). The general barrel field pattern was similar across the intact and trimmed vibrissae representations in PMBSF within and between hemispheres of $\mathrm{EtOH}$ and $\mathrm{CF}$ rats. Scale bar $=500 \mu \mathrm{m}$. 
Table 2.5 PMBSF areas in EtOH-trim, EtOH-intact, CF-intact, and CF-trim groups.

\begin{tabular}{lcccc}
\hline Treatment & $\begin{array}{c}\text { EtOH-trim } \\
(n=14)\end{array}$ & $\begin{array}{c}\text { EtOH-intact } \\
(n=14)\end{array}$ & $\begin{array}{c}\text { CF-intact } \\
(n=13)\end{array}$ & $\begin{array}{c}\text { CF-trim } \\
(n=13)\end{array}$ \\
\hline PMBSF area $\left(\mathrm{mm}^{2}\right)$ & $4.27^{\mathrm{a}}(0.06)$ & $4.40(0.07)$ & $4.54(0.07)$ & $4.29(0.08)$ \\
PMBSF barrel area $\left(\mathrm{mm}^{2}\right)$ & $2.68(0.04)$ & $2.75(0.05)$ & $2.76(0.04)$ & $2.63(0.05)$ \\
PMBSF septal area $\left(\mathrm{mm}^{2}\right)$ & $1.59^{\mathrm{a}}(0.05)$ & $1.65(0.05)$ & $1.78(0.06)$ & $1.66(0.04)$ \\
\hline
\end{tabular}

Data are presented as mean \pm SEM. A significant difference between EtOH-trim and CFintact $={ }^{\mathrm{a}}(\mathrm{P}<0.05)$. A significant difference did not occur between any other groups. 


\subsubsection{Intraregional vulnerability in vibrissae trimmed $\mathrm{EtOH}$ and $\mathrm{CF}$ juveniles}

To answer the question of whether sensory experience played a role in producing the posterior to anterior asymmetry identified in juvenile rats, unilateral whisker trimming was performed in an attempt to equilibrate the effect of sensory experience on the anterior-to-posterior asymmetry in the PMBSF . Since only barrel D2 $[F(3,50)=$ $3.005, \mathrm{p}=0.039]$ was significantly reduced in the EtOH-trim group compared to CFintact group, and barrel B2 was significantly reduced in the CF-trim group compared to the EtOH-intact group $[\mathrm{F}(3,50)=3.115, \mathrm{p}=0.0343]$, these results did not support the hypothesis that differential sensory experience was responsible for the anterior-toposterior asymmetry previously reported in the PMBSF barrel areas of juvenile PAE rats.

\subsection{DISCUSSION}

We, and others, have reported that PAE altered the size of PMBSF in neonatal rats (Margret, et al., 2006b; Margret, et al., 2005b) and mice (Powrozek \& Zhou, 2005), but did not disrupt the barrel pattern with the exception that an occasional barrel was reported missing in mice. One goal of the present study was to determine whether the effect of similar gestational alcohol exposure from GD1 to GD20 was restricted to neonatal rats or whether PAE had a long-term effect on barrel field size in juvenile and adult rats. The mitochondrial stain, cytochrome oxidase, was used to visualize the PMBSF; barrels in this subfield are large with wide intervening septal spaces and are easily distinguishable from neighboring barrels. The important findings in the present study are: 1) PAE significantly reduced the total PMBSF area in juvenile and adult rats compared to non-alcohol treated rats, 2) PAE significantly reduced the areas of individual 
PMBSF barrels in juvenile and adult rats, 3) PAE did not significantly effect inter-barrel septal area of PMBSF in either juvenile or adult rats, 4) intra-barrel subfield asymmetries were observed within the PMBSF indicating that the area of anterior PMBSF barrels was more reduced than in posterior barrels following comparisons with respective CF barrels, 5) an asymmetry was identified in the transition from posterior arcs to seemingly more affected anterior arcs in juvenile and adult EtOH groups. 6) PAE significantly reduced body weight in juvenile rats in comparison with $\mathrm{PF}$ controls but not $\mathrm{CF}$, 7) $\mathrm{PAE}$ significantly reduced brain weights in the juvenile age group but not the adult group. 8) vibrissae trimming significantly reduced the PMBSF and septal areas between the EtOHtrim and CF-intact groups, and 9) sensory experience did not appear to play a role in the anterior-to-posterior asymmetry. These findings support the notion that the effect of PAE on the cortical barrel field persists into adulthood, while the effect of PAE on body and brain weights diminish over time. However, the role of sensory experience in producing the posterior to anterior asymmetry in PMBSF of EtOH juvenile rats compared to CF rats remains unclear.

\subsubsection{PAE has a long-term effect on the somatosensory cortex}

Previously we used intragastric gavage to administer a high dose of alcohol (6 $\mathrm{g} / \mathrm{kg}$ ) to pregnant rat dams during the first twenty days of gestation and reported reductions in: body and brain weights, PMBSF area, PMBSF barrel area, and PMBSF septal area in rat pups at PD10 (Margret, et al., 2005b). Since body, brain, and cortical representation were all reduced, results indicated that PAE exerted a global reduction in brain and body weight in addition to a specific effect on somatosensory cortex (i.e., 
posterior barrels were less reduced compared to anterior barrels). However, a recent study in mice showed that alcohol administered over a limited period of gestation (GD8 to GD18; typical gestation length is 19 days) reduced the size of the PMBSF. Although body or brain weights of mice treated with EtOH were less than nutritional controls, the reductions were not statistically significant (Powrozek \& Zhou, 2005). These results suggested that gestational alcohol had a direct effect on barrel field cortex that was not confounded by concomitant reductions in body and brain weights. In the present study, body and brain weights and PMBSF area were compromised in the juvenile EtOH group, while only the overall size of the PMBSF and area of individual barrels were significantly reduced in the adult EtOH group. Interestingly, the septal region between barrels was not significantly reduced in either juvenile or adult rats, in contrast to findings in vibrissae trimmed juvenile EtOH rats and in previous studies on neonates (Margret, et al., 2005b). The asymmetrical/differential effect of PAE on barrels versus septa may be attributed to the differences in afferent input between septae (Gil, Needleman \& Huntley, 2002) and barrels (Molnar, Higashi \& Lopez-Bendito, 2003; Senft \& Woolsey, 1991) as well as possible differences in cortical circuitry, explained in greater detail below.

In support of the reductions in the areas of PMBSF barrels, PAE has also been shown to reduce: the overall volume of the somatosensory cortex, volume of individual cortical layers, number of cells within lamina, and total glial cell number (Miller \& Potempa, 1990); as well as produced ultrastructural changes in somata of layer V corticospinal neurons (al-Rabiai \& Miller, 1989). In addition, vibrissae stimulation altered glucose utilization in PAE rats at approximately 12 weeks of age (Miller \& DowEdwards, 1993). PAE has also been reported to reduce the thickness of layer V and 
cortical packing density but increased the thickness of layers II/III in 6-week old juvenile rats (Fakoya \& Caxton-Martins, 2006). These findings, along with the present results support the notion that PAE has a long-term effect on somatosensory cortex, and specifically on the cortical barrel field.

\subsubsection{PMBSF asymmetries in juveniles and adults}

\subsubsection{Asymmetric variability within the CNS}

It is well established that PAE produces general asymmetries in many locations, whereby some brain regions are affected while others are spared. For example, PAE administered during neurogenesis of each respective region (Maier \& West, 2003) reduced the number of cells in the locus coeruleus (LC), but not cerebellar purkinje cells or inferior olive cells. PAE significantly reduced CA1 hippocampal pyramidal cells without a concomitant reduction in CA2 through CA4 pyramidal cells or dentate gyrus granule cells (Barnes \& Walker, 1981; Gibson, et al., 2000). Cell number and volume of ventrobasal thalamus (Mooney \& Miller, 1999), and ventral lateral thalamus neurogenesis (Livy, et al., 2001) were unaffected in pups exposed to alcohol during neurogenesis, even though thalamocortical afferent inputs to cortical barrels were reportedly delayed (Margret, et al., 2005a). In contrast, cortical thickness (Fakoya \& Caxton-Martins, 2006), neuronal number (Miller, 1987), glucose utilization (Miller \& Dow-Edwards, 1988), neuronal migration (Miller, 1993) and neural cell adhesion molecules (Minana, et al., 2000) were disrupted in rats exposed to gestational alcohol. 


\subsubsection{Asymmetries within the PMBSF following PAE}

In this study, the PAE-induced reductions in individual PMBSF barrel area in both juvenile and adult groups are not uniform across the PMBSF. Interestingly, following initial statistical analysis of PMBSF barrel areas, anterior barrels of the EtOH group were more often significantly reduced than were posterior barrels (Fig 2.2). Data calculated as a percent change from CF suggested an anterior-to-posterior asymmetry pattern. The PMBSF asymmetry suggested that anterior barrels were proportionally much smaller than were posterior barrels. An asymmetrical division was shown between anterior and posterior barrels (Fig 2.3) when the percent reduction of the EtOH barrel area was divided at the midpoint range between minimum and maximum difference. Lateral to medial running arcs indicated that the anterior half of the PMBSF was more reduced than the posterior half of the PMBSF. In addition to the previously reported asymmetry in PAE neonatal rats (Margret, et al., 2005b), this study demonstrates that the PMBSF asymmetries in the EtOH group is a long-term effect.

\subsubsection{Possible peripheral substrates influencing the anterior-to-posterior PMBSF asymmetry}

Previous peripheral examinations of rodent vibrissae, follicle-sinus complex (FSC) [i.e., vibrissae root], and transected infraorbital nerves in rodents that under went FSC removal, revealed numerous asymmetries throughout the periphery. The most obvious peripheral asymmetry lay in the lengths of the mystacial vibrissae themselves. The mystacial vibrissae are not only longer moving anterior-to-posterior along the face, but the vibrissae bases are also thicker along the same gradient (Brecht, Preilowski \& 
Merzenich, 1997). Due to longer lengths of posterior vibrissae, these may come in contact with environmental objects more readily than anterior, shorter vibrissae (Carvell \& Simons, 1990). Furthermore, the posterior vibrissae more frequently palpate distant objects during daily exploration while anterior vibrissae remain in a more protracted, rigid state (Carvell \& Simons, 1990). Current theories underlying these vibrissae behavioral patterns have suggested that the longer, less dense posterior "macrovibrissae" may be critically involved with spatial recognition and therefore used as distance detectors, whereas the short, numerous "microvibrissae" may be involved more with object recognition (Brecht, Preilowski \& Merzenich, 1997). Also, the varying vibrissae lengths across rows and arcs might produce vertical and horizontal location information. Vertical information is encoded by vibrissae arcs and decoded by the lemniscal (peripheral afferent information synapsing within layer IV barrels) system. Horizontal location information is encoded by rows and decoded by the paralemniscal (peripheral afferents synapsing within layer IV septa) system (Ahissar \& Arieli, 2001). These peripheral variables could be playing a role in producing the anterior-to-posterior PMBSF asymmetry in juvenile and adult EtOH groups. Thus, PMBSF asymmetries could be produced due to asymmetrical sensory experience and/or by way of a more active lemniscal system (barrels) that expand barrel borders into that of the paralemniscal system (septa).

Additional peripheral asymmetries that may also underlie the anterior-to-posterior PMBSF asymmetry occur in the FSC located at the base of the vibrissae. In contrast to the anterior FSC, posterior mystacial pad FSC are innervated by more axons (Welker \& Van der Loos, 1986). We hypothesized that PAE might alter peripheral mystacial 
innervation patterns during development, which in part could disrupt postnatal barrel development. If this were the case, permanent changes could occur in the barrel field if proper peripheral sensory innervation was not restored immediately after birth. This is because peripheral tactile input during the first five postnatal days has been shown to be critical for proper barrel formation (Wong-Riley \& Welt, 1980). If PAE differentially affects anterior and posterior mystacial pad axonal innervation during pre- or early postnatal development, this might lead to the barrel asymmetries in neonatal, juvenile and adult rats.

Removal of the mystacial FSC was found to cause an asymmetrical pattern in the reinnervation of FSC (Melzer \& Smith, 1998a; Melzer \& Smith, 1998b), as posterior follicles were reinnervated sooner than anterior vibrissae follicles. Posterior FSC fibers were significantly longer than anterior FSC fibers at 64 days post-follicle removal. The difference in posterior and anterior follicle lengths was significantly correlated with asymmetric metabolic changes identified in the barrel cortex at a similar post-follicle removal time point (Melzer \& Smith, 1998a; Melzer \& Smith, 1998b). These studies suggested that whisker reinnervation and barrel metabolic activity are linked and that both contain anterior-to-posterior asymmetries. Similar regeneration patterns have been identified in other laboratories that have crushed or transected the infraorbital nerve and examined reinnervation of Merkel cells, the Ruffini complex, free nerve endings and lanceolate receptors (Renehan \& Munger, 1986). Furthermore, follow-up studies revealed that follicular reinnervation is row specific, running from anterior-to-posterior (Bronchti, Corthesy \& Welker, 1999; Corthesy, Bronchti \& Welker, 1999). Finally, control rats also 
have developmental asymmetries as innervating Merkel cells develop in a caudorostral orientation beginning GD15 (Nurse \& Farraway, 1988).

In a recent study, the effect of alcohol exposure during PD2 to PD10 on peripheral sensory receptors of rat forepaw was examined. Alcohol exposure reduced nociception [mechanical hypoalgesia and thermal hyperalgesia] (Sweitzer, et al., 2006). The investigators hypothesized that the effect of alcohol exposure could be due to peripheral as well as central alterations in the processing of nociceptive sensory information. Any serious PAE investigation must attempt to reconcile the influence of peripheral sensory receptors and subsequent sensory input on CNS and vice versa.

Posterior vibrissae have lower $(60 \mathrm{~Hz}$ to $100 \mathrm{~Hz})$ fundamental resonance tuning frequencies than anterior $(\sim 750 \mathrm{~Hz})$ vibrissae (Neimark, et al., 2003). These peripheral resonance patterns produce frequency maps between posterior and anterior barrels allowing the "tuning" of frequency signals that may assist in texture discrimination. A similar effect occurs in the auditory system, in particular the inner ear's cochlea with its ability to discriminate a wide range of sound frequencies. The differences in vibrissa resonance form "isofrequency bands" from posterior to anterior PMBSF arcs (Andermann, et al., 2004). As of yet, it is unknown whether this pattern in isofrequencies would influence postnatal barrel development, or whether PAE might perturb normal vibration frequencies.

Any one of the above mechanisms could act as a substrate for the PMBSF asymmetry identified in this study. In addition to the periphery, there are numerous CNS asymmetries that may also subserve the production of anterior-to-posterior asymmetries in the PMBSF. 


\subsubsection{Possible CNS substrates influencing the anterior-to-posterior PMBSF}

\section{asymmetry}

Cortical barrel asymmetries have been identified in: metabolic (McCasland, et al., 1991) and electrical activity levels (Riddle, et al., 1993), developmental patterns

(McCandlish, Li \& Waters, 1993; Rice \& Van der Loos, 1977; Stettler, Tavitian \& Moya, 1996), inhibitory summation (Brumberg, Pinto \& Simons, 1996), cortical thickness, and the relationship between peripheral axons and barrel areas (Welker \& Van der Loos, 1986). However, the most obvious central asymmetry is in the size of layer IV PMBSF barrels, as larger, more cell-dense barrels are found in posterior PMBSF while barrels typically become smaller (due, in part, to fewer cells) shifting towards anterior barrels. Studies on the vibrissae-to-barrel system indicated that longer vibrissae were associated with larger, more cell dense cortical barrels (Lee \& Woolsey, 1975; Welker \& Van der Loos, 1986).

Metabolic activity patterns were viewed following an injection of 2-deoxyglucose (2DG) into rats that were then allowed to freely roam cages, after which time they were sacrificed, perfused, brain tissue was sectioned, and then stained. In these animals, there was a greater glucose uptake in posterior barrels than in anterior barrels (Durham \& Woolsey, 1985; McCasland, et al., 1991; Shin, et al., 2005). Similar results were identified following vibrissae denervation (Bronchti, Corthesy \& Welker, 1999). The differences in metabolic activity levels across PMBSF may be due to greater surround inhibition in anterior barrels compared to posterior barrels (McCasland, et al., 1991). Furthermore, 2DG studies identified a directional row bias indicating that dendritic branching preferentially travels along rows in a posterior to anterior direction. Activity 
expansion following peripheral deafferentation of vibrissae input travels most prominently in a dorsal-to-ventral direction (Bernardo, McCasland \& Woolsey, 1990; Bernardo, et al., 1990; Shin, et al., 2005).

The PMBSF asymmetry could also be due to biochemical and/or physiological factors that accompany normal development and not necessarily due to PAE. Although the anterior barrels are always smaller than posterior barrels in normal rodents, the anterior barrels increase in size more than do posterior barrels during postnatal brain development (Riddle, et al., 1992). Thus the asymmetrical effect in the PMBSF of PAE rats may actually reflect an increase in anterior barrels of the $\mathrm{CF}$ group. However these results would hardly explain the similar PMBSF asymmetry identified in PAE rats at PD10 (Margret, et al., 2005b). Then again, rodent "whisking” does not begin until approximately PD15 so (Landers \& Philip Zeigler, 2006) the regional effect seen in PD10 neonatal rats is probably not due solely to active whisking behavior, but perhaps is more dependent upon differential follicle and Merkel cell development, rostrocaudal innervation pattern and regeneration asymmetries occurring in the mystacial pad (Brecht, Preilowski \& Merzenich, 1997; Melzer \& Smith, 1998a; Nurse \& Farraway, 1988; Welker \& Van der Loos, 1986).

Future studies are needed to better understand whether PAE has effects on afferent fibers and the mechanoreceptors of mystacial vibrissae and intervibrissal fur (Mosconi \& Rice, 1993; Munger \& Rice, 1986; Rice, Mance \& Munger, 1986; Rice \& Munger, 1986). If so, how may these peripheral influences further compound the direct effect of PAE on PMBSF cortical areas? 
Our results in juveniles and adults (non-trimmed) suggest either the possibility of recovery following PAE (in the case of posterior barrels), or that PAE inhibits normal postnatal development (in the case of anterior barrels). It is likely that PAE affects and disrupts each level of the biological system, from the developing mystacial pad to the development of vibrissae, axons, and mechanoreceptors of the periphery to generation and differentiation of the neurons, glia, and astrocytes necessary for the formation of barrel cortex.

\subsubsection{The effect of PAE and vibrissae trimming on the area of the PMBSF and septa}

The present study showed that unilateral vibrissae trimming has no significant effect on PMBSF area, PMBSF barrel area, septal area, or individual barrel area in the hemispheres of either trimmed or intact EtOH and CF groups. Vibrissae trimming only produced a significant effect in two barrels between treatment groups (barrel B2 of CFtrim was significantly smaller than EtOH-intact and barrel D2 was significantly smaller in EtOH-trim compared to $\mathrm{CF}$-intact). These results suggest that unilateral vibrissae trimming may not be an adequate method to eliminate sensory experience to produce a significant effect between cortical hemispheres within trimmed EtOH or CF rats, much less between trimmed EtOH and $\mathrm{CF}$ rats. Perhaps a more severe form of infraorbital nerve transection would produce a greater reduction in sensory input from the mystacial

pad to the contralateral hemisphere in both $\mathrm{EtOH}$ and $\mathrm{CF}$ rats. Another possibility is that peripheral experience is not important for producing an asymmetry in PMBSF barrel area. However, PMBSF area and septal area were significantly reduced in the EtOH-trim group compared to the CF-intact group suggesting that the combination of PAE and 
trimming significantly altered the area of the PMBSF. Because the area of the PMBSF in the EtOH-intact group was not significantly different than any other group (CF-intact, CF-trim, or the EtOH-trim) suggests that a larger sample size may be necessary to reach a significant effect between intact and trimmed hemispheres of trimmed EtOH and CF rats.

The significant reduction in PMBSF septa area of the EtOH-trim group is an interesting result, as a significant reduction was not previously identified in the results of non-trimmed juvenile rats of the current chapter. While the results from the trimmed EtOH rats and the non-trimmed rats cannot be compared directly, due to methodological considerations (alcohol habituation procedure and differences in flattening apparatus between the two groups of animals), the reduced septa area does suggest the role of sensory experience in producing and maintaining normal septal development.

Inter-barrel septa and the barrels of layer IV receive vibrissae information from two separate pathways [lemniscal and paralemniscal pathways] (Kim \& Ebner, 1999; Koralek, Jensen \& Killackey, 1988). Information traveling along the lemniscal pathway ascends in "barrel columns" from the ventral posterior medial thalamus (VPM) while information traveling along the paralemniscal system ascends in "septal columns" via the posterior medial nucleus of the thalamus (POm). There are reciprocal projections between VPM and cortical layers V/VI (Chmielowska, Carvell \& Simons, 1989; Lu \& Lin, 1993). POm projects to layers I and V and to the septa between barrels in layer IV while also receiving afferent fibers from layers V/VI (Diamond, 1995; Koralek, Jensen \& Killackey, 1988). This suggests that the thalamocortical loops are largely separate for these two pathways and opens the door for reasons explaining the necessity for these two systems (Ahissar \& Arieli, 2001; Kim \& Ebner, 1999). Regardless of the exact purpose 
for separate pathways, definite functional differences occur between information traveling along the lemniscal pathway and the paralemniscal pathway. The paralemniscal pathway has peripheral to central representation latencies that are more variable, the vibrissae responses in layer IV septa are weaker following peripheral vibrissae stimulation, and the receptive field sizes are larger in this system (Ahissar, Sosnik \& Haidarliu, 2000; Armstrong-James \& Fox, 1987; Armstrong-James, Fox \& Das- Gupta, 1992; Diamond, et al., 1992). Furthermore, the cortico-cortical projections from septal columns extend much further into surrounding cortex compared to the projection patterns from barrel columns (Kim \& Ebner, 1999). Some investigators have described the paralemniscal system in terms of its role in processing vibrissae information indirectly via the output from the lemniscal pathway (Diamond, Armstrong-James \& Ebner, 1992; Hoogland, Welker \& Van der Loos, 1987). This would suggest that the POm, sending information along the paralemniscal pathway, may function as a higher order nuclei by processing the output of the primary cortex (Kaas \& Ebner, 1998). While a greater review of septa versus barrel differences is beyond the scope of this study (Ahissar \& Arieli, 2001; Kim \& Ebner, 1999), future studies will need to further examine and address the role of EtOH on septal development in vibrissae trimmed and non-trimmed animals.

\subsubsection{Body and brain weights of juveniles and adults}

In the present study, the body weight of juvenile rats in the EtOH group was significantly lower than the body weight of rats in PF group. In contrast, no significant differences in body weights occurred between any adult groups, suggesting a catch-up with age. Other investigators have also reported significant reductions in body weight 
following fetal alcohol exposure in rats at four weeks of age (McMechan, et al., 2004) while no significant difference in PAE rats was found at 12 weeks (Miller \& Potempa, 1990), 16 weeks (Miller \& Dow-Edwards, 1988; Mooney, Napper \& West, 1996), or at 21 to 28 weeks (Savage, et al., 2002) of age, suggesting the potential for catch-up in body weight following PAE. These results in the body weights of rodents are interesting in light of reported reductions in body weight, height (Streissguth, et al., 1991), and bodymass index (Day, et al., 2002; Klug, et al., 2003) of children and adolescents with FASD. However, the differences in these parameters become negligible as the children reach adulthood; suggesting possible amelioration by environmental factors (Nordstrom-Klee, et al., 2002).

The present study also showed that whole brain and forebrain weights were significantly lighter in non-trimmed juvenile rats, while the brain weights of adult rats were not significantly affected by PAE. Supporting studies indicated that PAE $(5.8 \mathrm{~g} / \mathrm{kg})$ reduced brain weight at 6 weeks (Fakoya \& Caxton-Martins, 2006), 12 weeks (Miller \& Potempa, 1990) and 16 weeks (Miller, 1987; Mooney, Napper \& West, 1996) of age but not after 16 weeks of age (Allan, et al., 1998; Savage, et al., 2002). Results from the brain weights of the adult EtOH group in combination with previous results in the brain weights of EtOH, PF, and CF neonates (Maier, et al., 1997; Maier, Miller \& West, 1999; Margret, et al., 2005b) and juveniles indicates that significant brain weight differences disappear as $\mathrm{EtOH}$ rats reach adult age.

One hallmark feature of children whose mothers consumed alcohol during pregnancy is microcephaly (Clarren, et al., 1978; Ferrer \& Galofre, 1987) that typically persists through adolescence and into adulthood (Sowell, et al., 2001; Sowell, et al., 
2002a). In the present study, even though the mean brain weight of PAE adults was not significantly different from controls, the mean brain weights of both juvenile and adult rats exposed to alcohol during gestation were always lower than non-alcohol controls.

\subsection{CONCLUSION}

In the current study, PAE diminished total PMBSF area, and individual barrel area in six-week-old (juvenile) rats, an effect that persisted into seven-month-old (adult) rats. These effects of gestational alcohol on the body weight in juvenile and adult rats were vital in explaining earlier results in neonatal rats and suggested that the barrel cortex is reduced not merely because of smaller brain and body weights.

Our results in juvenile and adult rats are consistent with the effect of PAE seen in children indicating that amelioration of some physical traits such as the craniofacial malformations (Burd, et al., 2003a) as well as cortical deficits may be possible over time. Results from EtOH and CF rats that received unilateral vibrissae trimming suggested that a more severe form of sensory deprivation may be necessary to examine the effect of sensory experience on the posterior to anterior asymmetry identified in neonatal, juvenile, and adult $\mathrm{EtOH}$ rats. However, it is also possible that the periphery is unnecessary for producing the asymmetry in the barrel areas of the PMBSF. 


\section{CHAPTER 3}

\section{PRENATAL ALCOHOL EXPOSURE REDUCED THE AREA OF THE GLABROUS FOREPAW AND FOREPAW BARREL SUBFIELD}

\subsection{INTRODUCTION}

Prenatal alcohol exposure (PAE) in children can cause brain and body deficits that often persist to adulthood (Burd, et al., 2003b; Connor, et al., 2006; Jones \& Smith, 1973; Jones \& Smith, 1975; Riley \& McGee, 2005). Some of the physical deficits that occur in humans following PAE include shortened fingers, flexion contracture of the digits (camptodactyly), curving of the fingers (clinodactyly), and extra digits [polydactyly] (Pauli \& Feldman, 1986; Spiegel, et al., 1979). Similar limb defects such as extra digits with webbing situated between the digits (polysyndactyly), missing digits [ectrodactyly] (Kotch, et al., 1992; West, et al., 1981), or shorter digit lengths and lower digit ratios (McMechan, et al., 2004) have been described in animals exposed to high blood alcohol levels during gestation. In addition to limb deficits, PAE also disrupted the sensorimotor cortical system in humans, an effect that may lead to deficits in spatial and fine motor control (Kaplan-Estrin, Jacobson \& Jacobson, 1999), and delayed fine motor skills (Kalberg, et al., 2006). Imaging studies showed that PAE reduced the area of the parietal cortex in humans (Sowell, et al., 2001; Sowell, et al., 2002a). Rodent PAE-models have provided valuable information regarding the underlying mechanisms for sensory deficits seen in humans exposed to alcohol during pregnancy. These deficits may be attributed to ultrastructural abnormalities in neurons within sensorimotor cortex and thalamo-cortical connections as reported in PAE rats (Minciacchi, et al., 1993; Popova, 1989). 
SI is composed of structures called barrels that are associated with the representation of the body surface. Within SI, PAE has been shown to reduce the area of the posterior medial barrel subfield (PMBSF) that is associated with the representation of the large mystacial vibrissae in 9-day-old neonatal rats (Margret, et al., 2005b), 6-weekold juvenile rats, and 7-month-old adult rats (Chappell 2007, in press). Fewer neurons were found in PMBSF barrels in 6-week-old PAE pups compared to controls (Chappell, et al., 2005). PAE also significantly reduced the area of the forepaw barrel subfield (FBS) in neonatal rat pups (Margret, et al., 2006a), but whether this reduction persists in juvenile (six-weeks-old) rats is unknown.

In the present study, the effect of PAE on the area of the glabrous forepaw and the representation of the forepaw in the FBS were investigated in juvenile PAE rats. Our results suggest that PAE continues to exert long-term deficits on the size of the central representation of the forepaw in the FBS, while PAE produces little if any influence on the area of the glabrous peripheral forepaw in juvenile rats.

\subsection{METHODS}

\subsubsection{Animals}

Sprague-Dawley pups $(\mathrm{n}=51)$ from 24 dams were used in this study. All females were handled and habituated daily to the dry gavage procedure 4 days prior to breeding. 


\subsubsection{Breeding and treatment groups}

Breeding consisted of placing 1 to 3 adult female rats in a cage overnight with an adult male breeder. Vaginal smears were examined the next morning under a microscope for presence of sperm. Gestational day one (GD1) was marked as the day the female was sperm positive. On GD1 all pregnant dams were weighed, and separated into the following groups: alcohol treatment $(\mathrm{EtOH}, \mathrm{n}=9)$, pairfed $(\mathrm{PF}, \mathrm{n}=6)$, and chowfed $(\mathrm{CF}$, $n=9$ ). PF mothers were matched in weight to EtOH dams. Pregnant dams were separated into individual cages during pregnancy and pup rearing.

\subsubsection{Treatment procedures}

All daily procedures (handling of dams, gavaging solutions, food allotment provided each group, recording of dam weight and food consumption, and gavage needles used), blood sampling, blood alcohol level (BAL) analysis, postnatal rearing of pups, and tissue processing in this experiment were as described in Chapter 2 of this dissertation.

\subsubsection{Quantitative FBS morphometric analysis}

Digitization of sections, tissue reconstruction, measurements, and data analysis were as described in Chapter 2. Briefly, the FBS was delineated by placing a boundary line around the total FBS. The total area of FBS included the representations of P1, P2, P3, HT, and TH pads along with the ventral and dorsal representations of digits 2 (D2vd) to D5vd and D1. The glabrous digit representation was delineated by placing a line 
circumscribing D2v to D5v as shown in Fig 3.1 $\mathrm{A}^{\prime}$ to $\mathrm{C}^{\prime}$. These areas were measured with Image J (version 1.34s).

\subsubsection{Forepaw measurements}

Each animal was deeply anesthetized with a lethal dose of Nembutal $(100 \mathrm{mg} / \mathrm{kg})$. Following areflexia, forepaws were removed at the wrist joint and ventral surfaces of the forepaws were photographed (Fig 3.2) using an upright microscope. ImageJ was used to calculate the total surface area of glabrous forepaw as demarcated in Fig 3.2A. Individual glabrous digits (D2 to D5) were similarly outlined as shown in Fig 3.2B

\subsubsection{Statistical analysis}

Statview (version 5.1) was used for all statistical analyses. When the images of two hemispheres from the same animal were considered satisfactory, the areas of FBS representations on both hemispheres were measured and averaged. The cortical areas of FBS representation were compared across groups and compared using a one-way ANOVA with level of significance $(\alpha)$ set at 0.05 followed by pairwise comparisons using Tukey-Kramer's post-hoc test with significance level set at $\alpha=0.05$. When data from the EtOH group was significantly different from respective CF or PF data, the EtOH data was calculated as a percent change from the CF or PF group. 

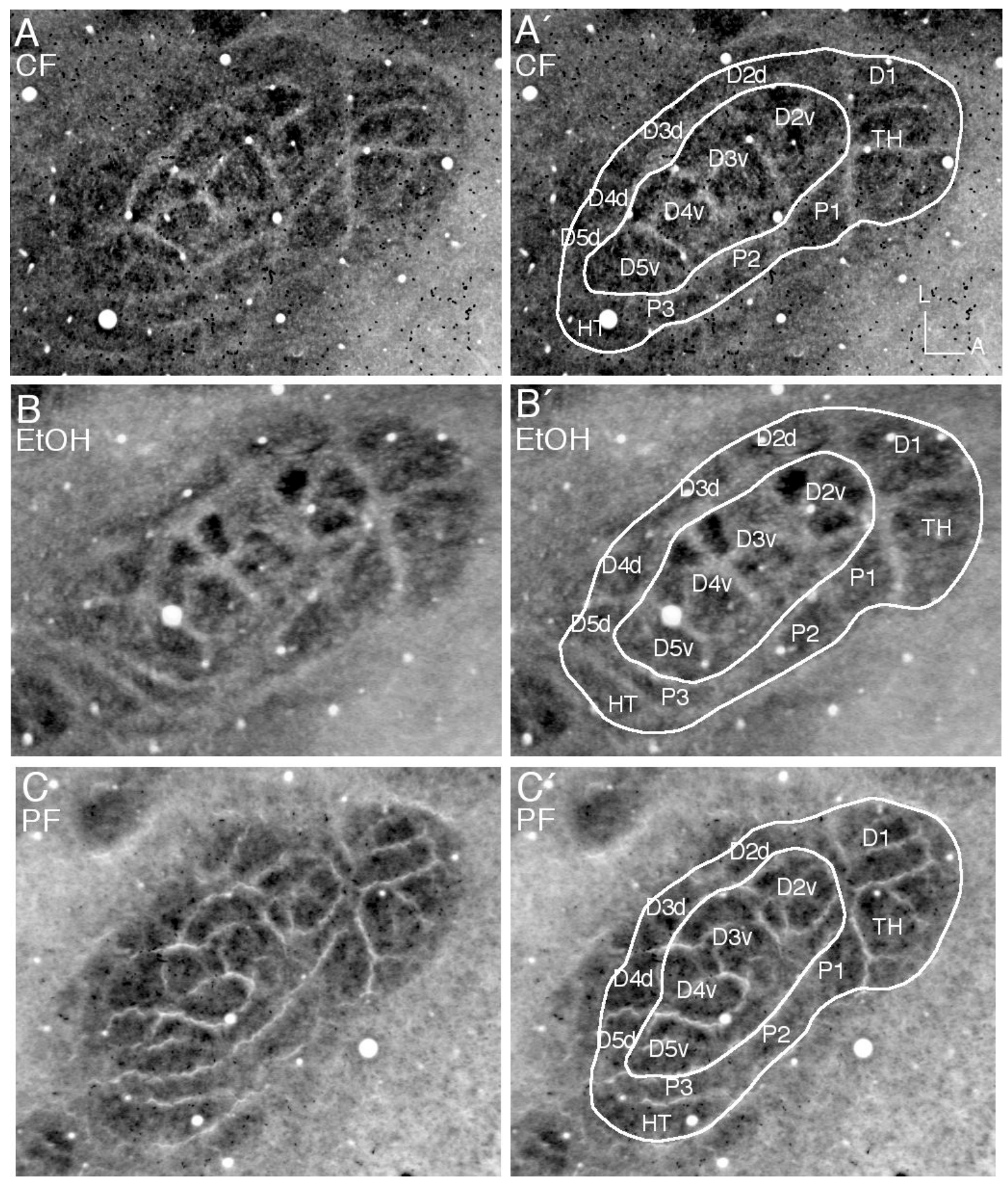

Figure 3.1 Total FBS and ventral digit representation for each group.

The forepaw barrel subfield (FBS) pattern illustrated for each group. A, $\mathbf{A}^{\prime}$ ), The total FBS in chowfed [CF], $\mathbf{B}, \mathbf{B}^{\prime}$ ) ethanol [EtOH], and $\mathbf{C}, \mathbf{C}^{\prime}$ ) pairfed [PF] groups (outerline), and ventral digit (D2v to D5v) representation (inner-line) Nomenclature: $\mathrm{D}=$ digit, $\mathrm{TH}=$ thenar pad, $\mathrm{P}=$ pad, $\mathrm{HT}=$ hypothenar pad, $\mathrm{d}=$ dorsal, $\mathrm{v}=$ ventral. 

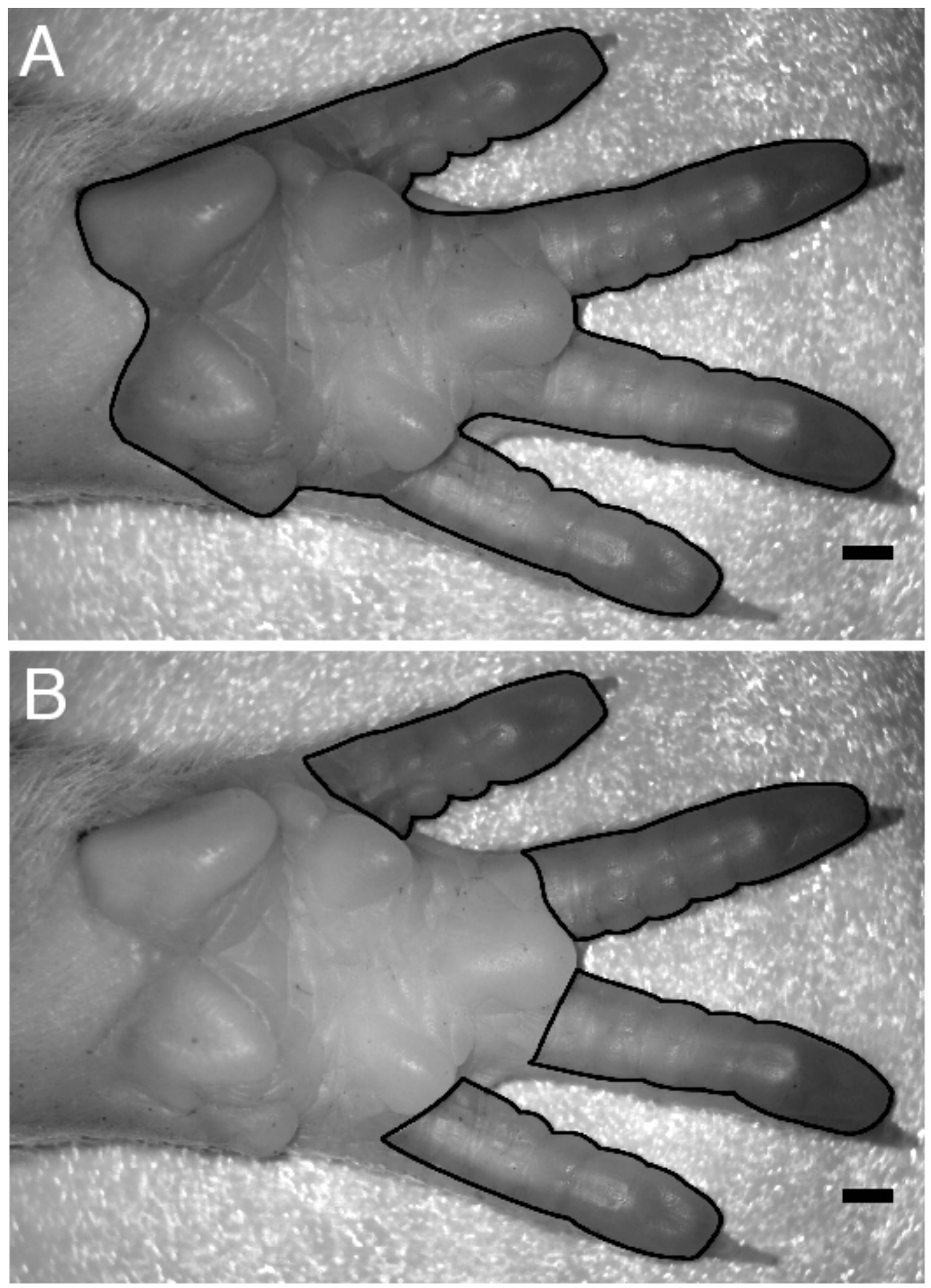

Figure 3.2 Glabrous forepaw and digit areas.

A) Outline of measured glabrous forepaw. B) Outline of measured glabrous digits, D2 to D5. Scale bar indicates $1 \mathrm{~mm}$. 


\subsection{RESULTS}

\subsubsection{Maternal variables}

The mean peak BAL in the EtOH group was $278.6 \pm 16.2$ (range; 210.4 to 353.4 $\mathrm{mg} / \mathrm{dl}$ ) on GD13, and 319.1 \pm 16.6 (range; 207.4 to $396.0 \mathrm{mg} / \mathrm{dl}$ ) on GD20 (Table 3.1). No significant difference occurred in mean weight gain between dams at GD1 and GD20 $[F(2,21)=2.129, p=0.1439]$ as shown in Table 3.1. No significant difference $[F(2,21)=$ 2.953, $\mathrm{p}=0.0741]$ in litter size of dams occurred between the groups (Table 3.1).

\subsubsection{Offspring variables}

\subsubsection{The effect of $P A E$ on the area of the FBS}

FBS area was significantly smaller in the EtOH group compared to both $\mathrm{CF}$ and PF groups $[F(2,48)=7.3, p=0.0018]$. When the data from the EtOH group was calculated as a percent difference from CF or PF groups, results indicated that the total FBS area of the EtOH group was $12.1 \pm 1.9 \%$ reduced compared to $\mathrm{CF}$ and $10.0 \pm 2.0 \%$ smaller than the PF group. No significant differences occurred between $\mathrm{CF}$ and PF groups (Table 3.2).

\subsubsection{The effect of PAE on the area of D2v to D5v barrels in FBS}

The area of D2v to D5v barrels was significantly reduced in EtOH group compared to $\mathrm{CF}$ and PF groups $[\mathrm{F}(2,48)=3.4, \mathrm{p}=0.042]$ as shown in Table 3.2. A significant difference between the EtOH and $\mathrm{CF}$ group was identified and $\mathrm{EtOH}$ was 
Table 3.1 Maternal variables.

\begin{tabular}{lccc}
\hline Treatment & EtOH $(n=9)$ & PF $(\mathrm{n}=6)$ & CF $(\mathrm{n}=9)$ \\
\hline Weight gain GD1 to GD21 $(\mathrm{g})$ & $95.1(11.5)$ & $100.3(8.0)$ & $119.7(6.4)$ \\
Litter-size & $11.1(1.4)$ & $15.2(0.7)$ & $12.3(0.8)$ \\
BAL on GD13 & $278.6(16.2)$ & NA & NA \\
BAL on GD20 & $319.1(16.6)$ & NA & NA \\
\hline
\end{tabular}

Data presented as mean \pm SEM for weight gains, litter size, and BAL of mothers (on GD13 and GD20) of the rats used in this experiment. No significant differences occurred between the groups.

\section{Table 3.2 PAE reduced the area of the FBS.}

\begin{tabular}{lcc}
\hline Treatment & Total FBS mm$^{2}$ & D2v to D5 $\mathrm{vm}^{2}$ \\
\hline $\mathrm{EtOH}(n=17)$ & $1.67^{\mathrm{a}, \mathrm{b}}(0.04)$ & $0.63^{\mathrm{a}}(0.02)$ \\
$\mathrm{PF} \quad(n=17)$ & $1.87(0.05)$ & $0.67(0.02)$ \\
$\mathrm{CF} \quad(n=17)$ & $1.90(0.05)$ & $0.70(0.02)$ \\
\hline
\end{tabular}

Data presented as mean $\pm \mathrm{SEM}$. A significant difference between groups indicates a Pvalue of $(\mathrm{P}<0.05)$. EtOH versus $\mathrm{CF}={ }^{\mathrm{a}}$, EtOH versus $\mathrm{PF}={ }^{\mathrm{b}}$. 
reduced $10.2 \pm 1.6 \%$ compared to the $\mathrm{CF}$ group. However, no significant difference occurred between EtOH and PF groups or between PF and CF groups.

\subsubsection{Effect of PAE on the area of the glabrous forepaw}

\subsubsection{Total glabrous forepaw area}

The total area of glabrous forepaw was significantly different between groups $[\mathrm{F}(2,48)=3.8, \mathrm{p}=0.03]$. The EtOH group had a significantly smaller glabrous forepaw than $\mathrm{CF}$, but not PF group (Table 3.3). The data revealed that the glabrous forepaws from the EtOH group were $6.8 \pm 1.8 \%$ smaller than $\mathrm{CF}$ group.

\subsubsection{Glabrous digit area}

ANOVA revealed a significant effect in the forepaw glabrous digits D2 to D5 $[F(2,48)=5.6, p=0.0064]$. PAE significantly reduced the size of glabrous forepaw digits

2 to 5 (Table 3.3) compared to the CF group, not the PF group. The glabrous digit area of the EtOH group was $7.7 \pm 1.6 \%$ smaller in the EtOH group compared to the CF group. However, unlike previous studies that examined neonatal and in utero PAE-pups, no missing digits, fused digits, or extra digits were found in the forepaws of any EtOH rat examined in the present study.

\subsection{DISCUSSION}

The purpose of this study was to evaluate whether the changes in forepaw size and forepaw representation reported in neonatal rats (Margret, et al., 2006a) following 
Table 3.3 PAE and the glabrous forepaw.

\begin{tabular}{lcc}
\hline Treatment & Total glabrous forepaw $\left(\mathrm{mm}^{2}\right)$ & Glabrous digits 2 to $5\left(\mathrm{~mm}^{2}\right)$ \\
\hline EtOH $(n=17)$ & $93.86^{\mathrm{a}}(1.81)$ & $42.52^{\mathrm{a}}(0.72)$ \\
$\mathrm{PF} \quad(n=17)$ & $95.78(1.68)$ & $44.41(0.75)$ \\
$\mathrm{CF} \quad(n=17)$ & $100.72(1.96)$ & $46.10(0.78)$ \\
\hline
\end{tabular}

Data are presented as mean \pm SEM. A significant difference between groups indicates a $\mathrm{P}$-value of $(\mathrm{P}<0.05)$. EtOH versus $\mathrm{CF}={ }^{\mathrm{a}}$. 
PAE (GD1 to GD20) persisted in six-week old rats. This period of PAE coincides with the first and second trimesters in humans and is also a critical period for neurogenesis, cell proliferation and cell migration during cortical development.

By using cytochrome oxidase, we were able to visualize and measure the cortical barrels representing the overall glabrous forepaw and the glabrous digits. Our major findings were that PAE significantly reduced the areas of the following: 1) FBS, 2) ventral digit representation D2 to D5, 3) peripheral glabrous forepaw, and 4) glabrous peripheral digits 2 to 5 compared to the CF group. However, the only difference between EtOH and PF groups occurred in the total FBS representation. These findings support the hypothesis that PAE-induced deficits in total FBS size previously reported in neonates continue in juvenile rats. However, the area of the FBS and forepaw area appear equally affected by PAE, nutrition, and stress. Our major findings are : 1) PAE significantly reduced the area of the FBS. However, PAE, nutrition, and stress played roles in altering 2) the D2 to D5 representation in the FBS, 3) the total glabrous forepaw, and 4) the area of the glabrous digits D2 to D5.

\subsubsection{FBS area}

The present study demonstrated that PAE altered the morphological representation of the forepaw in the FBS. These findings support the changes in the brain that have been reported by other investigators who studied the effects of PAE on the brain. These changes include: significant reductions in the numbers of neuronal cell bodies, glia, and calbindin neurons (Granato, 2006), as well as significant increases in the size of the soma of glia (Miller \& Potempa, 1990) and the number of calretinin neurons 
(Granato, 2006), while PAE also increased $\mathrm{GABA}_{\mathrm{A}}$ receptors (Bailey, Brien \& Reynolds, 2004).

PAE also has been documented to induce changes within the rodent somatosensory cortex. For example, PAE significantly reduced: the number of neurons in the barrels of layer IV of somatosensory cortex (Chappell, et al., 2005; Powrozek \& Zhou, 2005), the area of the posterior medial barrel subfield in neonate (Margret, et al., 2005b), juvenile, and adult rats (Chappell 2007, in press), and the area of the FBS representation in the cortex of neonatal rats (Margret, et al., 2006a). The findings in these previous studies strongly support the persistent reduction in the total area of the FBS of juvenile rats reported in this chapter.

A compromised somatosensory cortex could lead to deficits in sensorimotor information processing and fewer connections to motor cortex where sensory information is involved in the control and modulation of motor movement. Furthermore, increased sensory processing times (described in Chapter 5) may lead to impaired premotor responses often associated with the teratogenic effect of PAE, as exhibited in children exposed to prenatal alcohol (Simmons, et al., 2002).

The fact that the area of the D2 to d5 digit representation in the FBS of the EtOH group was significantly reduced from CF rats but not significantly reduced from the PF group suggests that nutrition is also playing a role in reducing the digit representation. Of interest is the fact that the average area of the digit representation is more greatly reduced in the EtOH group than in the PF controls suggesting that more than nutritional affects are responsible for the reduced representation. It is also interesting to note that the PF and CF groups are not significantly different from one another, although the average digit 
representation is smaller in the $\mathrm{PF}$ groups than in the $\mathrm{CF}$ group. We interpret this to suggest that it is not nutrition alone that accounts for the reduced forepaw representation. Nonetheless, our results are puzzling that the EtOH group indicates a significant reduction in the total forepaw representation in the FBS, while this reduction does not carry over to the representation of individual digits. It is possible that region specific differences may play a role in facilitating a greater reduction in the presence of PAE, or that some cortical regions are more readily ameliorated following PAE in juvenile rats. As previously reported in chapter 2 , not all barrel arcs and rows in the posterior medial barrel subfield were significantly reduced between the EtOH group and either the CF or PF groups. The lack of a significant effect in some cortical regions may be due to activity dependence, however this hypothesis has yet to be proven. The possibility exists that the representation of glabrous digits 2 to 5 may recover to a greater extent over time given that the glabrous forepaw likely palpates the environment more frequently.

\subsubsection{Glabrous forepaw and total glabrous digit area}

The PAE-induced reduction in forepaw size compared to $\mathrm{CF}$ in juvenile rats extends our previous findings in neonates where PAE was also compared to CF rats (Margret, et al., 2006a). For example, the areas of the glabrous forepaw and glabrous forepaw digits 2 to 5 were significantly reduced in neonatal (PD9) rats that were exposed to alcohol during gestation. However, because a significant reduction did not occur in the present study between the EtOH group and the PF group, we can not suggest that PAE significantly reduced the glabrous forepaw, rather that a combination of PAE, reduced nutrition, stress of daily handling, and blood sampling all played roles in this effect. 
Furthermore, since no correlations were found between peripheral glabrous forepaw and the FBS of neonates (Margret, et al., 2006a), and between brain weight and PMBSF of juvenile rats (Chapter 2), we did not examine the relationship between FBS and glabrous forepaw or between FBS and brain weight in the present study.

The effect of PAE on the periphery has been well documented in humans as well as animals. Children of alcoholic mothers often show growth deficits (Burd, et al., 2003a; Jones \& Smith, 1973; Jones, et al., 1973) that include shorter digits in children (Jaffer, Nelson \& Beighton, 1981) and adolescence (Habbick, et al., 1998). The use of animal models has shown that PAE leads to digit malformations (West, et al., 1981), fused and missing digits (Fadem, et al., 1989), forepaw digit ectrodactyly and syndactyly (Chrisman, et al., 2004; Kotch, et al., 1992) and shortened digits (McMechan, et al., 2004) in animals.

The influence of PAE on digit development may be due, in part, to reactive oxygen species (ROS) and non-ROS (Johnson, Blanton \& Hunter, 2004) related mechanisms influencing the limb bud during development by increasing apoptotic cell death and mesenchymal tissue loss of the developing limbs' apical ectodermal ridge (Chrisman, et al., 2004). One study in rats incorporated the antioxidant superoxide dismutase in combination with alcohol and reported that ROS played a role in the malformation of developing limb buds. In this study, the group that received antioxidants and alcohol had fewer limb malformations compared to groups that received alcohol alone (Chen, Dehart \& Sulik, 2004). A similar effect was also seen in mouse embryos that were exposed to alcohol plus antioxidants or alcohol alone (Kotch, Chen \& Sulik, 1995). Strong correlations have also been identified between PAE and cell death patterns 
in embryonic regions related to limb-bud formation. The cell death in the apical ectodermal ridge (location of future limbs) was observable within 8 to $12 \mathrm{hrs}$ following an initial alcohol insult when rat fetuses are examined (Cartwright \& Smith, 1995; Dunty, Zucker \& Sulik, 2002; Kotch \& Sulik, 1992). However, while others have indicated PAE-related effects on digit formation, the lack of significant effects between our EtOH and PF groups in the size of the glabrous forepaw or glabrous digits suggests that the effect of PAE alone does not produce significant reductions in the peripheral forepaw in juvenile rats. Unless the EtOH group is significantly different from both the $\mathrm{CF}$ (control) and PF (nutritional and stress control) groups, it is impossible to determine whether an effect is due to PAE, or the reduced caloric intake and stress of handling involved with the dosing paradigm.

We previously reported in neonatal rats that digit length as well as the digit representation in the FBS were significantly reduced in PAE pups (Margret, et al., 2006a). However, in neither neonatal nor juvenile groups were digit malformations ever identified nor was an unorganized FBS ever observed, indicating that our dosing paradigm was either not severe enough to produce digit malformations, or that pups with digit malformations had significant PAE and nutrition related impairments to cause the reabsorbing of the fetus prior to delivery.

The present study examined the morphological representation of the forepaw (FBS) in rodents exposed to gestational alcohol to better understand whether the effect of PAE, previously examined in neonates, persisted in juvenile rats. In Chapter 4, we physiologically mapped the representation of the forepaw in somatosensory cortex and examined evoked response latencies between digits and cortex in PAE and non-PAE 
groups to determine whether PAE altered the physiological representation of the forepaw and disrupted conduction latency between periphery and cortex. 


\section{CHAPTER 4}

\section{PRENATAL ALCOHOL EXPOSURE REDUCED THE AREA OF THE FOREPAW REPRESENTATION IN SOMATOSENSORY CORTEX}

\subsection{INTRODUCTION}

Fetal Alcohol Spectrum Disorder (FASD) is a non-diagnostic term used to describe the broad range of effects following prenatal alcohol exposure [PAE] (Goodlett, Horn \& Zhou, 2005; Riley \& McGee, 2005). Deficits in postural balance (Roebuck, et al., 1998a; Roebuck, et al., 1998b), higher-order cognitive motor tasks (Adnams, et al., 2001), reaction times (Simmons, et al., 2002), motor coordination (Bookstein, et al., 2002b), motor timing (Wass, et al., 2002), fine motor control (Connor, et al., 2006), and delayed fine motor skills (Kalberg, et al., 2006) have been reported in children with FASD. These findings strongly suggest the existence of abnormalities in brain structures responsible for sensorimotor information processing and integration (Bookstein, et al., 2002b). In support of these brain structural anomalies by prenatal alcohol exposure (PAE), imaging studies have shown that parietal cortex (Archibald, et al., 2001; Sowell, et al., 2001; Sowell, et al., 2002a; Sowell, et al., 2002b), the interhemispheric pathway between parietal cortices (Roebuck, Mattson \& Riley, 2002), and the parietal cortex to motor cortex pathway (Bookstein, et al., 2002b) are particularly susceptible to early alcohol exposure.

Animal models have been used to examine the effect of both PAE and postnatal alcohol exposure (PostAE) on sensorimotor cortex. Reduction in the area of large mystacial vibrissa barrel fields in the primary somatosensory cortex (SI) have been 
reported in rodents that were exposed to PAE or postnatal alcohol (Margret, et al., 2006b; Margret, et al., 2003; Margret, et al., 2006c; Powrozek \& Zhou, 2002; Powrozek \& Zhou, 2005). In contrast, the barrel pattern was relatively invariant. Similarly, PAE and PostAE have been documented to alter the physiological properties of SI vibrissae-related neurons and SI forepaw-related neurons. For example, in relation to SI, PAE was reported to reduce: spontaneous firing levels of neurons, evoked response levels, and long latency responses to whisker stimulation. PAE also altered whisker pairing (a form of cortical plasticity produced by cutting all but two mystacial vibrissae) in SI of three month old rats (Rema \& Ebner, 1999). Similarly, acute PostAE also reduced spontaneous firing and reduced evoked response levels in forepaw-related SI neurons in adult rats (Chapin, Sorensen \& Woodward, 1986). PAE also altered SI cortical firing levels during ethanol withdrawal (Chapin \& Woodward, 1989).

Functional electrophysiological studies have also been used to map the body representation in SI in rodents (Welker, 1976). Detailed maps of the forelimb representation reveal an orderly somatotopic organization of the forepaw, wrist, forearm, and shoulder (Pearson, Li \& Waters, 1999; Waters, McCandlish \& Li, 1995). SI forelimb neurons also receive short latency input from the periphery (Pearson, Li \& Waters, 1999). However, to date no study has examined the effect of PAE on the SI map of the forepaw representation, and latency of forepaw stimulation to SI. The purpose of the present study was to examine the effect of PAE on the physiological representation of the forepaw in SI and to examine the effect of PAE on evoked response latencies of SI forepaw neurons following tactile or electrical digit stimulation. Results from this study are relevant for 
understanding the mechanisms underlying the behavioral deficits in somatosensory function and reaction times reported in children with FASD.

\subsection{METHODS}

\subsubsection{Animals, breeding, and treatment groups}

A total of 27 Sprague-Dawley juvenile rats was used in this study. Following females $(n=21)$ and males being placed together overnight, vaginal swabs from each female were smeared on glass slides and examined under a microscope for sperm presence. Gestational day one (GD1) was defined as the first day sperm were present. Pregnant dams were weighed, separated into groups (alcohol [EtOH], $\mathrm{n}=6$, pairfed [PF], $\mathrm{n}=7$, chowfed $[\mathrm{CF}], \mathrm{n}=8$ ), and placed in individual cages.

\subsubsection{Treatment procedures}

The treatment procedures for: daily procedures (handling of dams, gavaging solutions, food allotment provided each group, recording of dam weight and food consumption, and gavage needles used), blood sampling, blood alcohol level (BAL) analysis, and postnatal rearing of pups used in this experiment were as described in Chapter 2 of this dissertation.

\subsubsection{Surgery}

At six weeks of age, rats were injected with sodium pentobarbital (35 mg/kg, i.p.) and supplemented by $10 \%$ of initial dose as necessary in order to maintain areflexia. The 
scalp was shaved, head placed in a stereotaxic apparatus, a midsagittal slit was made in the overlying skin, and tissue was infused with $3 \%$ Polocaine (mepivacaine HCL). The skull was drilled open to expose somatosensory cortex and the cisterna magna opened to alleviate cranial pressure. A chamber was formed around the exposed cortex using dental cement, the dura was then removed and the chamber was filled with silicone fluid (10,000 centi-stokes). A digital photomicrograph was made of the brain surface and the image viewed on a computer monitor. The location of electrode penetrations was marked on the image for plotting the receptive fields of the forepaw representation in SI cortex.

\subsubsection{Physiological mapping and map reconstruction of primary receptive fields in the forepaw representation of SI}

The methods used to produce electrophysiological maps of the forepaw representation have been previously described (Coq \& Xerri, 1998; Hall \& Lindholm, 1974; Li, et al., 1996; Waters, Li \& McCandlish, 1995; Xerri, et al., 1996). In brief, single or multi-unit cortical responses were recorded from neurons in SI using a carbon fiber electrode attached to a Narishige microdrive. The electrode was inserted into SI at a depth $700 \mu \mathrm{m}$ to record receptive fields of neurons. This depth has been previously shown to lie within the central portion of layer IV of SI in the forepaw representation (Waters, Li \& McCandlish, 1995). The electrophysiological signal was amplified and then fed into a storage oscilloscope and audio monitor. A hand-held probe consisting of a thin wire fastened onto a wooden applicator stick was used to lightly tap the skin surface and the responses to tapping were recorded in SI cortex (Coq \& Xerri, 2000; Hall \& Lindholm, 1974). The primary receptive field was defined as the area of skin that yielded 
a maximal response with minimal tactile stimulation. While multiple digit and pad representations were occasionally identified in any one penetration, only the primary receptive field/s or the region of forepaw that produced the greatest response to tactile stimulation was recorded in the present study. Electrode penetrations were spaced at approximately $150 \mu \mathrm{m}$ from one another, but varied depending on surface vasculature. Typically, 90 to 110 electrode penetrations were necessary to generate a complete map of the forepaw representation.

The receptive field of each electrode penetration was projected to the cortical surface and used to generate the map of the forepaw representation. The map was transferred into a drawing program (Clarisdraw) and partitioned into individual digit and pad representations by drawing boundaries around penetration sites that enclosed receptive fields for digits and pads. Boundary lines were drawn equi-distant between penetrations yielding different primary receptive fields and through penetration sites with overlapping primary receptive fields (Coq \& Xerri, 2000). The receptive field areas for digits and pads were measured and quantified using NIH image and Image J (courtesy of Wayne Rashbaum).

\subsubsection{Evoked response latencies}

At the conclusion of mapping, evoked response latencies were measured in SI. A bipolar electrode consisting of a pair of twisted, silver, chloride wires, attached to a probe, was used to deliver current to the tips of digit 2 (D2) to D4. Stimulation consisted of a single square wave pulse $(0.1 \mathrm{~ms}$ duration, $1 \mathrm{sec}$ inter-pulse interval, $1.5 \times$ threshold, 
$60 \mu \mathrm{A}$ maximum current amplitude) delivered to the tip of the digit. Evoked response latencies were collected for 50 consecutive stimulations and averaged (RC Electronics).

\subsubsection{Statistical analyses}

Data analysis was performed with Statview (version 5.1). The data for the three groups were compared using a one-way ANOVA. Differences were considered significant at $\alpha \leq 0.05$, followed by Tukey-Kramer's post-hoc test (significance at $\alpha \leq$ 0.05). When EtOH data were significantly different from measurements in either $\mathrm{CF}$ or PF groups, this data was calculated as a percent difference from the respective group.

\subsection{RESULTS}

\subsubsection{Maternal blood alcohol levels}

Blood samples were taken on GD13 and GD20. On GD13, EtOH dams had a mean peak blood alcohol level (BAL) of $257.7 \pm 18.2$ (range; 210 to $327 \mathrm{mg} / \mathrm{dl}$ ), while the mean peak BAL on GD20 was $322 \pm 21.3$ (range; 207 to $396 \mathrm{mg} / \mathrm{dl}$ ).

\subsubsection{Effect of PAE on the forepaw representation in SI}

The rat forepaw consists of glabrous and dorsal hairy skin surfaces with four wellformed digits (digit two [D2], D3, D4, and D5) and a stunted D1. Three digit pads and two palmar pads are also found on the glabrous surface. The forepaw is somatotopically represented in SI and this is illustrated in Fig 4.1. The forepaw map in SI is ovoid in shape. This pattern is seen across all groups. Within each map, all parts of the glabrous 
and dorsal skin surfaces are represented somatotopically with the glabrous surfaces of ventral digit $2(\mathrm{D} 2 \mathrm{v})$ to $\mathrm{D} 5 \mathrm{v}$ located centrally, while digit 1 (D1) is represented at the anterolateral edge of the forepaw representation. The three digit pads (pad one [P1], P2, and P3) are located medially and bordered by thenar (TH) and hypothenar (HT) pads.

The dorsal hairy digit surfaces form the lateral border of the forepaw representation map. Examples of forepaw maps from EtOH, PF, and CF groups are also shown in Figure 4.1. Note that each map contained all digit and pad representations and the general pattern of the forepaw representation in SI is identical across the groups.

\subsubsection{Effect of PAE on the total ventral forepaw representation}

The total ventral forepaw representation was significantly different between groups $[F(2,24)=4.768, p=0.0181]$. The total ventral forepaw representation was significantly smaller in the EtOH group compared to CF and PF groups; no significant difference occurred between CF and PF groups (Table 4.1). The total glabrous forepaw representation in the EtOH group was reduced $13.9 \pm 4.4 \%$ compared to $\mathrm{CF}$ group while EtOH was $15.8 \pm 4.3 \%$ smaller than PF.

\subsubsection{Total forepaw representation}

The total forepaw representation was significantly different between groups $[F(2,24)=7.558, p=0.0028]($ Table 4.1). The dorsal hand $(\mathrm{Hd})$ was not included in the map since the borders were often ambiguous and difficult to map. The total forepaw representation was significantly smaller in the EtOH group compared to both $\mathrm{CF}$ and $\mathrm{PF}$ groups. However, no significant difference resulted between PF and CF groups. The total 

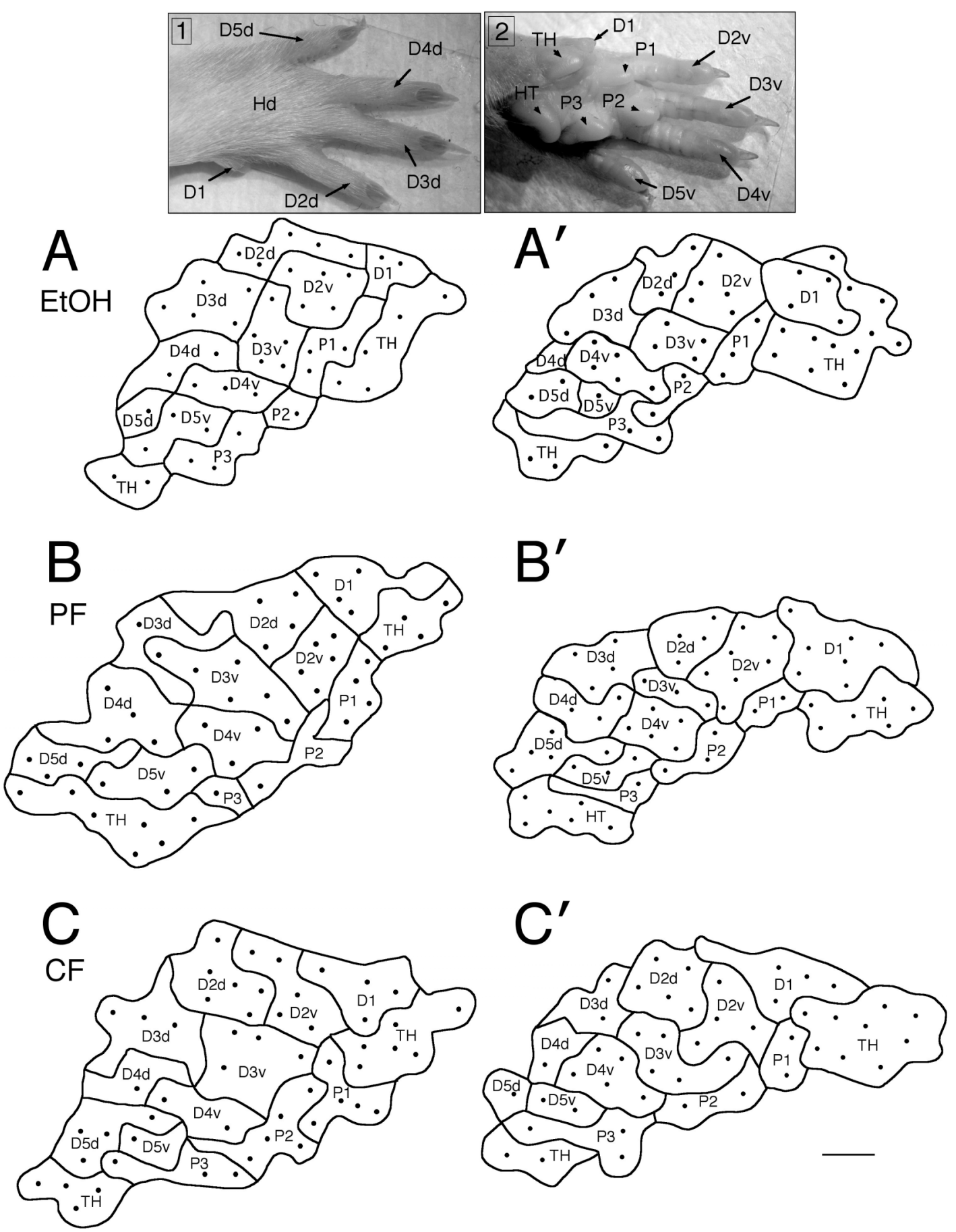

Figure 4.1 Forepaw representation of each group.

$\mathbf{A}, \mathbf{A}^{\prime}$ ) Examples of forepaw maps in SI for two EtOH rats. B, $\mathbf{B}^{\prime}$ ) Two PF maps. $\mathbf{C}, \mathbf{C}^{\prime}$ ) Two CF maps. Dots within maps represent penetration sites. The maps are somatotopically organized and the common pattern can be observed in each group. RFs were examined at $700 \mu \mathrm{m}$ below the cortical surface. Inset shows a photomicrograph of the rat forepaw with nomenclature used in generating forepaw maps. Scale $=250 \mu \mathrm{m}$. 
Table 4.1 Electrophysiological forepaw representations.

\begin{tabular}{lccc}
\hline Treatment & EtOH $(n=7)$ & CF $(n=10)$ & PF $(n=10)$ \\
\hline Total ventral forepaw representation $\left(\mathrm{mm}^{2}\right)$ & $1.07^{\mathrm{a}, \mathrm{b}}(0.05)$ & $1.24(0.04)$ & $1.26(0.05)$ \\
Total forepaw representation $\left(\mathrm{mm}^{2}\right)$ & $1.38^{\mathrm{a}, \mathrm{b}}(0.04)$ & $1.68(0.07)$ & $1.64(0.05)$ \\
D2vd to D5vd representation $\left(\mathrm{mm}^{2}\right)$ & $0.76^{\mathrm{a}}(0.02)$ & $0.93(0.04)$ & $0.90(0.04)$ \\
D2v to D5v representation $\left(\mathrm{mm}^{2}\right)$ & $0.43^{\mathrm{b}}(0.02)$ & $0.49(0.01)$ & $0.50(0.01)$ \\
D2d to D5d representation $\left(\mathrm{mm}^{2}\right)$ & $0.32^{\mathrm{a}}(0.03)$ & $0.46(0.03)$ & $0.38(0.02)$ \\
D2vd representation $\left(\mathrm{mm}^{2}\right)$ & $0.23^{\mathrm{a}}(0.01)$ & $0.30(0.02)$ & $0.27(0.01)$ \\
D3vd representation $\left(\mathrm{mm}^{2}\right)$ & $0.19^{\mathrm{a}, \mathrm{b}}(0.02)$ & $0.26(0.02)$ & $0.26(0.01)$ \\
D4vd representation $\left(\mathrm{mm}^{2}\right)$ & $0.19(0.01)$ & $0.21(0.02)$ & $0.20(0.02)$ \\
D5vd representation $\left(\mathrm{mm}^{2}\right)$ & $0.15(0.01)$ & $0.18(0.02)$ & $0.16(0.02)$ \\
\hline
\end{tabular}

Data presented as mean $\pm \mathrm{SEM}$. A significant effect for $\mathrm{EtOH}$ versus $\mathrm{CF}={ }^{\mathrm{a}}$, EtOH versus $\mathrm{PF}={ }^{\mathrm{b}}(\mathrm{P}<0.05)$. 
forepaw representation was reduced by $17.9 \pm 2.2 \%$ in the $\mathrm{EtOH}$ group compared to the CF group and $16.1 \pm 2.2 \%$ smaller in the EtOH group compared to the PF group.

\subsubsection{D2vd to D5vd representation}

The total ventral and dorsal digits representation (D2vd to D5vd) was significantly different between groups $[\mathrm{F}(2,24)=3.867, \mathrm{p}=0.035]$ (Table 4.1). The EtOH group had significantly smaller D2vd to D5vd area compared to the CF group, but not the PF group. No significant difference occurred between CF and PF groups. The EtOH group was $17.7 \pm 2.7 \%$ smaller than the CF group.

\subsubsection{D2v to D5v representation}

The area of the dorsal (d) digit representation (D2d to D5d) was significantly different between groups $[F(2,24)=4.465, p=0.0225]$. The $\mathrm{D} 2 \mathrm{v}$ to $\mathrm{D} 5 \mathrm{v}$ representation in the EtOH group was significantly smaller compared to PF but not CF groups (Table 4.1) although a trend to significance was identified. No differences occurred between CF and PF groups. The area of the ventral representation in EtOH was reduced by $11.6 \pm 4.7 \%$ compared to $\mathrm{CF}$ and $13.7 \pm 4.6 \%$ compared to $\mathrm{PF}$.

\subsubsection{D2d to D5d representation}

There were significant differences between groups in dorsal digit area representation (D2d to D5d) $[F(2,24)=4.465, p=0.0225]$. This area was significantly reduced in $\mathrm{D} 2 \mathrm{~d}$ to $\mathrm{D} 5 \mathrm{~d}$ of rats in the EtOH group compared to the CF group, but was not significantly different between EtOH and PF groups (Table 4.1). No significant 
differences were found between $\mathrm{CF}$ and PF groups. Interestingly, the dorsal area resulted in the greatest reduction $(29.5 \pm 5.9 \%)$ in EtOH compared to the CF group.

\subsubsection{Individual digit representations}

A significant main effect was found between $\mathrm{D} 2 \mathrm{vd}[\mathrm{F}(2,24)=5.224, \mathrm{p}=0.0131]$ and $\mathrm{D} 3 \mathrm{vd}[\mathrm{F}(2,24)=5.639, \mathrm{p}=0.0098]$ digits, while no significant main effect occurred in D4vd or D5vd area between groups. The area of the D2vd representation was significantly smaller in EtOH compared to the CF group but not to the PF group, while the area of the D3vd representation was significantly smaller in the EtOH group compared to the PF and the CF groups (Table 4.1). D2vd and D3vd of the EtOH group were reduced by $21.6 \pm 3.9 \%$ and $26.2 \pm 7.2 \%$ compared to CF respectively, while the area of D3vd digit representation in EtOH was $25.7 \pm 7.2 \%$ smaller than the respective digit in the PF group. We found no significant differences in D2vd, D3vd, D4vd, or D5vd representations between the CF and the PF groups.

\subsubsection{Evoked response latencies}

Since no significant differences in evoked response latencies recorded from D2, D3 or D4 were found within an individual group, latencies from all digits within a group were combined and analyzed (EtOH, $n=12 ; \mathrm{CF}, \mathrm{n}=17 ; \mathrm{PF}, \mathrm{n}=17)$. Evoked response latencies were found to be significantly different in D2 to D4 between groups $[F(2,43)=$ $10.02, \mathrm{p}=0.0003]$ (Table 4.2). The total combined latencies were significantly longer in the EtOH and the PF groups compared to latencies in the CF group. Latencies for the EtOH group were $9.3 \pm 1.7 \%$ longer than latencies in the CF group. 
Table 4.2 Longer evoked response latencies occurred in the EtOH and PF groups.

\begin{tabular}{lccc}
\hline Treatment & EtOH $(n=12)$ & CF $(n=17)$ & PF $(n=17)$ \\
\hline Summed D2v-D4v Latencies (msec) & $9.4^{\mathrm{a}}(0.3)$ & $8.5(0.2)$ & $9.5^{\mathrm{b}}(0.2)$ \\
\hline
\end{tabular}

Data presented as mean \pm SEM. A significant difference between groups indicated by: EtOH versus $\mathrm{CF}={ }^{\mathrm{a}}$, $\mathrm{CF}$ versus $\mathrm{PF}={ }^{\mathrm{b}}(\mathrm{P}<0.05)$. 


\subsection{DISCUSSION}

The purpose of this study was to examine the effect of PAE ( $6 \mathrm{~g} / \mathrm{kg}$ from GD1 to GD20) on the physiological representation of the forepaw in juvenile rats and peripheralto-CNS evoked response latencies. PAE reduced the overall area of the total forepaw representation in SI in juvenile PAE rats, but did not alter the general somatotopic organizational representation of the forepaw in SI. The evoked response latencies between periphery and central representation of $\mathrm{EtOH}$ and $\mathrm{PF}$ groups were significantly increased compared to the CF group. The main findings in this study were: PAE significantly reduced the representative areas of the total ventral forepaw, total forepaw and D3vd.. A smaller cortical representation may contribute to the numerous deficits in learning, balance, reaction times, and other sensorimotor-type disruptions frequently seen in children with FASD.

Our results indicate that the significantly reduced values between EtOH and CF groups may not be solely an effect of PAE per se, but may actually be an affect due to nutrition and stress of handling pregnant dams. Therefore, the PF control group becomes very important for our PAE data. As a reminder, the PF dam received an isocaloric/isovolumetric maltose dextran solution and only the amount of food eaten by a matched EtOH dam on the equivalent gestational day. Furthermore, PF dams also received identical stress as the $\mathrm{EtOH}$ dam when blood samples were taken from each dam during gestation. It must be emphasized that the PF group is also a treatment group that must be realized when interpreting results from this study. For example, PF rats may experience heightened levels of stress in comparison to the EtOH dams when blood is sampled by way of EtOH's analgesic abilities. Furthermore it is possible that the 
metabolic rate of PF dams may be much higher than $\mathrm{EtOH}$ dams due to the lethargic state of EtOH dams in the initial 2 to 4 hours following ethanol dose. No observable lethargy was observed in PF dams following maltose dextran treatment. This lowered activity level in EtOH dams may have allowed for their conservation of energy through less motility in comparison to PF dams. However, despite these minor weaknesses in the current study's methodology, the role of the PF treatment group is vital.

It should be noted that measurements from the PF group frequently fall midpoint between EtOH and CF results. When a significant difference occurs between EtOH and $\mathrm{CF}$ while no significant effect occurs between $\mathrm{EtOH}$ and $\mathrm{PF}$ is interpreted as an effect indicative of comorbid factors between PAE/nutrition/and stress, rather than purely a PAE effect as seen when EtOH is significantly different from both CF and PF groups. A more difficult interpretation occurs when EtOH is significantly different from PF but not CF groups. While this result is less frequently observed, it is usually accompanied with a trend towards a significant effect between EtOH and CF groups and may be due to inadequate sampling from the EtOH group. In those cases where EtOH and PF group measurements were similar and both groups were significantly different from the $\mathrm{CF}$ group, nutrition and stress likely produce the majority of the effect. This situation is occurred in the latency results seen in Table 4.2.

\subsubsection{PAE reduced the forepaw representation in juvenile rats}

Despite the extensive documentation of numerous sensorimotor deficits in children with FASD (Burden, Jacobson \& Jacobson, 2005; Church \& Abel, 1998; Janzen, Nanson \& Block, 1995; Kaplan-Estrin, Jacobson \& Jacobson, 1999; Roebuck, et al., 
1998b; Simmons, et al., 2002), this is the first study using a rodent model to determine if PAE disrupts the cortical representation of the body surface (forepaw) and periphery to CNS evoked response latency.

Our results indicating a significant reduction in 1) the area of the total forepaw and 2) total ventral forepaw representation between EtOH and both CF and PF groups are consistent with the findings of other investigators who reported a detrimental effect of PAE in the somatosensory cortex. For example, PAE altered the columnar cortical organization in visual cortex and disrupted sensory information processing (Medina, Krahe \& Ramoa, 2005), as well as EEG activity levels which may have led to the behavioral hyperactivity in the rats (Slawecki, 2002; Slawecki, et al., 2004), as well as the severely reduced spontaneous activity levels of neurons in barrel cortex; effects such as these persisted even in rats reared in an enriched environment (Rema \& Ebner, 1999). Interestingly, the enriched environment did not significantly increase the neuronal activity levels of these rats (Rema \& Ebner, 1999). This is surprising in light of other studies that resulted in the amelioration of some PAE-induced motor deficits from enriched environmental conditions (Thomas, et al., 1996).

In addition to the reduction in the area of the total forepaw representation in the EtOH group, the representation for D2d to D5d was more reduced ( 30\%) than in D2v to D5v $(\sim 18 \%)$ when data were calculated as a difference to respective areas in the CF group. Interestingly, the area of D2d to D5d was not significantly reduced between EtOH and PF groups, an effect suggesting nutrition may play a role in reducing cortical representations less susceptible to postnatal improvements. This results also suggest that the reduction in this representative area may not be due to PAE alone, but an interaction 
between PAE/nutrition/stress. Previous studies suggest that the difference in area between dorsal and ventral digit representations may be due to use-dependent plasticity (Maier, et al., 2003), in that the glabrous forepaw may receive more tactile stimulation in typical day-to-day activity behavior, thus providing more cortical activation in the corresponding SI ventral forepaw representation. This possibility is supported by results from studies indicating that PAE modifies behavioral activity levels by producing hyperactive animals (Riley, et al., 1993; Thomas, et al., 1998) and humans (Nanson \& Hiscock, 1990). Hyperactivity may lead to activity-dependent cortical plasticity between the ventral forepaw representation (possibly more metabolically and electrically active in a hyperactive animal that is palpating its environment more often) and the less stimulated dorsal forepaw representation.

The existence of a use-dependent plasticity in somatosensory cortex is supported by environmental enrichment studies where rats were housed in enriched environments that included two large cages ( $76 \mathrm{~cm}$ wide $\times 76 \mathrm{~cm}$ deep $\times 40 \mathrm{~cm}$ high) connected by two tunnels. Cages also included running wheels and numerous objects of different shapes, sizes, and textures to promote tactile stimulation. Objects were replaced daily with novel ones to promote exploratory behavior (Coq \& Xerri, 1998). Following $\sim 100$ days of environmental enrichment, the physiological representation of the area of the forepaw was significantly increased compared to animals reared in standard cage environments. Furthermore, the glabrous representation, not the dorsal hairy skin surface representation, showed the most increase in surface area when compared to representations in control rats raised in a standard caged environment. In contrast, when the forepaw was immobilized for seven days, producing a state of sensory deprivation to the forepaw, the 
physiological forepaw representation decreased by $\sim 50 \%$ and remained reduced even after 7 days of free limb movement was allowed (Coq \& Xerri, 1998; Xerri, et al., 1996). While not directly related to the present study, findings in these studies tend to provide explanations for decreases in the areas of D2v to D5v (by 12\%) and D2d to D5d (by $\sim 30 \%$ ) representation in SI cortex of PAE rats.

Possible morphological substrates leading to a reduced forepaw representation could be related to the effect seen in numerous studies that examined differential environmental influences on brain and body. For example, sensory/environmental deprivation resulted in lighter cerebral weights (Bennett, et al., 1974; Walsh, 1981), reductions in numbers of dendritic spines (Globus, et al., 1973) as well as decreased dendritic branching (Greenough \& Volkmar, 1973; Greenough, Volkmar \& Juraska, 1973). Environmental impoverishment also reduced the number of neuronal synapses (Greenough, Hwang \& Gorman, 1985; Sirevaag \& Greenough, 1985), perikarya volume (Diamond, et al., 1975) and mitochondria number (Black, Zelazny \& Greenough, 1991). In contrast, environmental enrichment [as recently reviewed (Churchill, et al., 2002; Nithianantharajah \& Hannan, 2006)] resulted in heavier cerebral and cortical weights (Ferchmin \& Eterovic, 1986), increased capillary branching and increased cortical surface area (Sirevaag, et al., 1988), more DNA (Ferchmin \& Eterovic, 1986) and RNA content in the brain (Ferchmin \& Eterovic, 1987) and more synapses per neuron (Sirevaag \& Greenough, 1988).

The possibility that the differentially decreased area of D2d to D5d representation reported here might be due to a reduction in peripheral tactile experience in both EtOH and PF groups needs to be explored in future studies. However, the present results are 
clearly not due to complete sensory denervation, as this would likely produce an expansion of representative forepaw areas into sensory deprived cortical regions. For example, when all mystacial vibrissae were removed except Row C, a progressive expansion into surrounding cortex occurred in the physiological vibrissae-related representation of the spared row (Kossut \& Juliano, 1999). In contrast, when less severe environmental impoverishment occurred, the area of skin receptive fields decreased in area (Coq \& Xerri, 1999).

\subsubsection{Increased evoked response latencies in EtOH and PF groups}

For central processing to work efficiently, signal transfer must be accurate and rapid between the periphery and central targets. Any delay of a signal in turn delays the ability to process information. Our results showed that evoked response latencies from the periphery to SI were delayed in EtOH and PF groups suggesting that both PAE and nutrition were involved in delayed signal processing. However, significantly longer latencies in $\mathrm{EtOH}$ rats, compared to $\mathrm{CF}$ rats, is similar to children with FASD, and emphasizes the importance of additional intrinsic (poor health and nutrition, genetics) and extrinsic (low socioeconomic status, culture) influences that often enhance the teratogenic effect of PAE (May, et al., 2004). It is likely that some brain regions and structures (such as neuronal myelination) may be more effected by PAE following additional concomitant variables such as poor nutrition (Church, et al., 1998). More importantly, these latency results corroborate previous research findings regarding difficulties in responding to visual and auditory stimuli onset in children with FASD (Kable \& Coles, 2004); slower reaction times in tasks involving: mental rotation, arrow 
discrimination, and memory scanning (Burden, Jacobson \& Jacobson, 2005); and an increased choice reaction time which was attributed to peripheral delays and reduced information processing speed (Simmons, et al., 2002). Reduced reaction times can be explained in part by reduced speed of signal passage traveling from periphery to central nervous system possibly due to PAE induced peripheral nerve pathology (Baruah \& Kinder, 1989) as alcohol retracts and shrinks axons. Despite Burden et al. (2005) concluding that reaction time deficits are found in the context of complex cognition and not in the processing of automatic responses, our data show that even automatic stimulation from the periphery to the CNS is delayed in both EtOH and PF groups. The delay in evoked response latencies from periphery to cortex may be due in part to reduced levels of axon myelination as shown in layer V cortex of PAE rats at PD30 (alRabiai \& Miller, 1989) and PD52 (Lancaster, et al., 1984).

Future studies must also examine the role of environmental conditions in producing many of the results produced in PAE studies. At least one environmental enrichment study suggests that evoked response latencies traveling from vibrissa to PMBSF were slower in rats in standard or impoverished environments and significantly faster for rats reared in an enriched environment (Seo, 1992). This study suggests that standard cage environments, while clearly not the natural habitat for rodents, may exacerbate deficits produced by PAE and poor nutrition. While we do not suppose that the environmental conditions where the rats from the current chapter were reared was anything less than "standard", when PAE and poor nutrition is coupled with a standard cage environment, it is possible that these three variables may concomitantly lead to 
longer evoked response latencies as well as reduced physiological forepaw representation areas in juvenile rats.

\subsection{Conclusion}

Considerable evidence now exists supporting the hypothesis that PAE has a short and long-term effects on rat somatosensory cortex (Margret, et al., 2005b; Miller \& DowEdwards, 1993; Powrozek \& Zhou, 2005). The deficits found in SI of juvenile rats in this study may be an important substrate for deficits in processing and learning in children identified with FASD (Burden, Jacobson \& Jacobson, 2005; Janzen, Nanson \& Block, 1995; Kaplan-Estrin, Jacobson \& Jacobson, 1999; Roebuck, et al., 1998b; Simmons, et al., 2002). Furthermore, the parietal lobe, containing SI, is severely effected following PAE (Archibald, et al., 2001). PAE has also been shown to reduce SI neurons (Miller \& Potempa, 1990; Powrozek \& Zhou, 2005) and produce severe motor coordination deficits which may be related to slower latencies and a diminished ability of the cortex to process information due to the general size reduction of the sensory cortex in animals and humans (Simmons, et al., 2002; Thomas, et al., 2000). Taken as a whole, these results underscore the potential long-term dangers that result in children whose mothers drink during pregnancy. 


\section{CHAPTER 5}

\section{THE EFFECT OF PRENATAL ALCOHOL EXPOSURE ON THE PRIMARY MOTOR CORTEX}

\subsection{INTRODUCTION}

Prenatal alcohol exposure (PAE) has been known to produce numerous teratogenic effects during human development (Hoyme, et al., 2005). Children exposed to gestational alcohol are categorized under the umbrella term of fetal alcohol spectrum disorder (FASD), a term covering the broad range of deficits that remain with the children throughout their lives.

Children with FASD are frequently reported to suffer from delayed motor development and fine-motor skill dysfunctions (Janzen, Nanson \& Block, 1995; Kalberg, et al., 2006; Kaplan-Estrin, Jacobson \& Jacobson, 1999) including hand tremors, weak hand strength, and poor hand-eye coordination (Riley \& McGee, 2005). They have also been shown to exhibit impaired performance on the Grooved Pegboard test, which measures the speed of fine motor movement and coordination (Mattson, et al., 1998). Children and adults with FASD performed more poorly than controls in balance and fine motor control tests that examined unilateral and bilateral finger, hand, and foot coordination (Connor, et al., 2006). Additional PAE-related behavioral and cognitive deficits in children include: posture (Roebuck, et al., 1998a), executive functioning (Mattson, et al., 1999), attention (Brown, et al., 1991), memory (Mattson \& Riley, 1999), and IQ (Mattson, et al., 1997). Finally, when children with FASD were tested using the Griffiths Mental Development Scale (used to assess cognitive development), the children 
had deficits in "higher-order cognitive-motor competencies" (Adnams, et al., 2001); an effect possibly due to an increased amount of time the children processed information (Simmons, et al., 2006).

PAE-related disruptions in posture and balance are likely due, in part, to deficits in the basal ganglia (Mattson, et al., 1994; Mattson, et al., 1996), cerebellum (Sowell, et al., 1996), and corpus callosum (Riley, et al., 1995; Roebuck-Spencer, et al., 2004). However, evidence now indicates that the primary motor cortex (MI) may play a role in higher-order executive functioning associated with learning and cognitive motor movements, as well as assisting in fine motor control (Donoghue \& Sanes, 1994; Sanes \& Donoghue, 2000). Therefore, the fine motor, learning, and planning deficits often observed in children with FASD suggest that PAE affects MI development. While only a few studies have examined the effect of postnatal alcohol on MI in humans (Kahkonen, et al., 2001), there is far less information on the effect of PAE on MI in monkey (Miller, 2006) or rodent (Slawecki, 2002) animal models.

The functional organization of rodent MI has been extensively studied by placing microelectrodes into MI and using intracortical microstimulation (ICMS) to elicit peripheral movements in rats (Donoghue \& Wise, 1982; Gioanni \& Lamarche, 1985; Hall \& Lindholm, 1974; Miyashita, Keller \& Asanuma, 1994; Neafsey, et al., 1986; Neafsey \& Sievert, 1982) and mice (Li \& Waters, 1991). These studies indicated that the movements of different parts of the body (forelimb, hindlimb, vibrissae, neck, tongue, jaw, and eyelids) are topographically arranged into discrete regions in MI (Gioanni \& Lamarche, 1985; Neafsey, et al., 1986). However, it is unknown whether PAE alters the organization of body parts in MI. 
We took advantage of the mototopic organization in MI to test the hypothesis that PAE alters the organization of forepaw and vibrissae representation in MI. We also hypothesized that PAE rats would require higher levels of activating current to elicit motor movements compared to control rats. These results may provide better understanding of the deficits in fine motor control (Mattson, et al., 1999) observed in children (Connor, et al., 2006; Janzen, Nanson \& Block, 1995; Kalberg, et al., 2006; Kaplan-Estrin, Jacobson \& Jacobson, 1999; Riley \& McGee, 2005) and adults (Connor, et al., 2006) with FASD.

\subsection{METHODS}

\subsubsection{Breeding of dams and treatment groups}

All virgin female Sprague-Dawley rats were dry-gavaged four consecutive days prior to breeding to habituate them to the gavage procedure. Breeding occurred by placing two virgin female rats in a cage overnight with a proven male breeder. Daily vaginal smears were examined for the presence of sperm. Gestational day one (GD1) was marked when sperm-positive identification occurred. Pregnant dams were weighed, and separated into one of three groups: alcohol $(\mathrm{EtOH}, \mathrm{n}=4)$, pairfed $(\mathrm{PF}, \mathrm{n}=2)$, or chowfed $(\mathrm{CF}, \mathrm{n}=3)$ groups. PF dams were matched in body weight $( \pm 10 \mathrm{~g})$ to an EtOH dam. Dams remained in separate cages during pregnancy and after delivery with their nursing pups until PD28. 


\subsubsection{Treatment procedures}

EtOH dams were gavaged daily from GD1 to GD20 with 25\% ethanol mixed with water. The dams were habituated to alcohol gavaging from GD1 to GD3, with $1 \mathrm{~g} / \mathrm{kg}, 3$ $\mathrm{g} / \mathrm{kg}, 5 \mathrm{~g} / \mathrm{kg}$ of alcohol respectively, then followed by $6 \mathrm{~g} / \mathrm{kg}$ of $25 \% \mathrm{w} / \mathrm{v}$ ethanol solution in water from GD4 to GD20. Also, PF dams that served as nutritional and stress controls were gavaged daily with an isocaloric and isovolumetric maltose dextran solution.

Furthermore, each PF dam was paired to an $\mathrm{EtOH}$ dam in that PF dams were allotted only the amount of food that their matched $\mathrm{EtOH}$ dam had eaten on an equivalent gestational day. CF dams served as normal controls and had no food restrictions. All dams in the three groups had access to water ad libitum.

Daily procedures consisting of food allotment to each respective group $(\mathrm{EtOH}$, $\mathrm{PF}$, and CF), recording of daily food consumption, weight gain of dam, needles used for gavaging, and the obtaining of blood alcohol levels, as well as parturition and rearing methods, were as described in Chapter 2. All experiments conformed to the Principles of Laboratory Animal Care (NIH publication No. 86-23, revised 1985) and were approved by the Animal Care and Use Committee, UTHSC. The Animal Care Facility is accredited by AAALAC.

\subsubsection{Surgery}

At six weeks of age, 18 rats (six from each examined group) were anesthetized with ketamine hydrochloride $(85 \mathrm{mg} / \mathrm{kg})$ and xylazine $(15 \mathrm{mg} / \mathrm{kg})$. A supplemental dose of ketamine/xylazine (10\%) was administered to maintain areflexia. Due to the highly dependent nature of MI maps on level of anesthesia, supplemental doses of 
ketamine/xylazine were administered only when spontaneous motor movements were observed. The scalp was shaved and the head placed in a stereotaxic apparatus. A local anesthetic (Polocaine (mepivacaine HCL)) was injected into the scalp. A scalp incision was made to expose the skull and the cisterna magna was opened to reduce cranial pressure. A small window was made in the cranium and the underlying dura mater was removed to expose the motor cortex. A chamber fashioned out of dental cement was formed around the exposed cortex. The chamber was filled with warm silicone fluid $(10,000$ centi-stokes) and the surface of the cortex was photographed. The image of the cortical surface was viewed on a computer and used to mark the location of electrode penetrations.

\subsubsection{Microstimulation and motor mapping}

A tungsten microelectrode (impedance of 1-3 $\mathrm{M} \Omega$ at $1 \mathrm{kHz}$ ), attached to a Narishige microdrive, was used to deliver ICMS. To reduce the possibility of variations in the stimulation physics (i.e., strength of actual level of stimulating current that could be diminished by electrodes coated with tissue) of the experiment, the electrode was cleaned prior to use, then lowered into the brain to an initial depth of $1400 \mu \mathrm{m}$. The microelectrode was used to deliver a train of cathodal stimulating pulses (12 pulses/train, $0.2 \mathrm{~ms}$ duration, $300 \mathrm{~Hz}$ ) through a constant current source (WPI model A360) to elicit motor responses. A search stimulus of $50 \mu \mathrm{A}$ was initially used to elicit motor responses; however, if an evoked movement was not observed, the stimulus amplitude was increased in $5 \mu \mathrm{A}$ steps until either a movement was elicited or a maximum current of $100 \mu \mathrm{A}$ was reached. Threshold for movement was determined by reducing the stimulating current 
until movement ceased, at that point, the current was slowly increased until movement reappeared with three consecutive stimulations. This stimulus amplitude was defined as threshold.

The maximum current for ICMS was capped at $100 \mu \mathrm{A}$ as this is a typical maximum stimulating current for the production of MI maps (Donoghue \& Wise, 1982; Gharbawie, et al., 2005; Hall \& Lindholm, 1974; Neafsey, et al., 1986). Furthermore, 100 $\mu \mathrm{A}$ produces a current spread roughly 250 to $300 \mu \mathrm{m}$ in radius from the stimulating electrode (Asanuma, Arnold \& Zarzecki, 1976; Tehovnik, et al., 2006). Current spread from a stimulating current of $100 \mu \mathrm{A}$ would travel approximately half the distance between adjacent electrode penetrations that were placed $\sim 500 \mu \mathrm{m}$ apart. Bregma was used as a zero reference point because it is considered to be the location of the most posterior portion of the forepaw motor representation (Hall \& Lindholm, 1974; Neafsey, et al., 1986). Microelectrode penetrations were aligned with Bregma, perpendicular to the surface of the cortex, and spaced approximately $500 \mu \mathrm{m}$ from one another depending upon vasculature of the cortical surface. ICMS was used to generate a motor map based on the lowest evoked response threshold $(\leq 100 \mu \mathrm{A})$ for forepaw and vibrissae movements obtained between depths of $1400 \mu \mathrm{m}$ and $1800 \mu \mathrm{m}$. ICMS was delivered at $100 \mu \mathrm{m}$ increments between depths of $1400 \mu \mathrm{m}$ and $1800 \mu \mathrm{m}$. These depths have been previously reported to lie within deep layer V and superficial layer VI without passing into underlying white fiber tracts (Brecht, et al., 2004a; Neafsey, et al., 1986). Map boundaries were determined when ICMS failed to elicit a motor response, elicited motor response with thresholds greater than $100 \mu \mathrm{A}$, or evoked movements other than from forepaw or vibrissae. Forepaw manipulation and stroking the vibrissae were used to 
achieve lower threshold intensities as previously described (Gioanni \& Lamarche, 1985;

Li \& Waters, 1991; Neafsey, et al., 1986).

\subsubsection{Motor cortex map reconstruction}

The movement elicited by the lowest stimulation current (threshold) for each penetration site was projected to a 2-dimensional grid superimposed on the cortical surface and served to define the motor receptive field for that penetration. Motor responsive sites for vibrissae and forepaw with ICMS thresholds $\leq 100 \mu \mathrm{A}$ were used to construct a motor map. Each data point showed the movement elicited by the lowest stimulating current, location of electrode penetration, and ICMS threshold current for that movement. Map boundaries were established by placing lines between penetration points when only one motor movement occurred at threshold (e.g. vibrissae versus forelimb), while boundary lines were superimposed upon penetrations representing two different motor areas of equal threshold. A secondary vibrissae representation was often identified posterior to the forelimb map and was always separated from the medial strip of vibrissae by either hindpaw (HP) responses, or a non-responsive (NR) penetrations. Only the area of the medial vibrissae was analyzed in this study.

\subsubsection{Measurements and statistical analysis}

Reconstructed motor maps were saved as TIFF files, and measured using ImageJ. Statview (version 5.1) was used to perform all statistical analyses. The data for the three groups were compared using a one-way ANOVA to determine if the groups were statistically different, followed by Fisher's post-hoc for pair-wise comparisons. The level 
of significance $(\alpha)$ was set at 0.05 . Size of the motor representation and ICMS threshold were calculated as percent changes in the values for the EtOH group compared to the values in the $\mathrm{CF}$ and $\mathrm{PF}$ groups, which were used as a standard control or nutritional control respectively. The mean threshold for each group was calculated by averaging the lowest stimulating threshold current from all penetrations used to produce a motor map for each animal respectively. The mean threshold was then used in the ANOVA and Fisher's post-hoc statistical analysis mentioned above.

\subsection{Results}

\subsubsection{Maternal blood alcohol level}

Blood samples were taken on GD10 and GD17. EtOH dam blood alcohol levels were $276.1 \pm 34.2 \mathrm{mg} / \mathrm{dl}$ (range; 169.2 to 353.4 ) on GD10 and $296.7 \pm 16.2 \mathrm{mg} / \mathrm{dl}$ (range; 248.2 to 330.8 ) on GD17.

\subsubsection{The effect of PAE on the area of forepaw and vibrissae representations in MI}

An example of a typical motor map in MI, transposed on the cortical surface, is shown in Fig 5.1 to demonstrate the location of MI on the cortical surface. Fig 5.1 shows the location of vibrissae motor representation in relation to the forepaw motor representation. A motor map consisting of ICMS thresholds $\leq 100 \mu \mathrm{A}$ is shown for each group in Fig 5.2. The general mototopic organization in MI was similar between groups. The vibrissae motor representation was located medially and ran rostral-caudally along a thin strip of cortex beginning near bregma and extending approximately $4 \mathrm{~mm}$ in the 


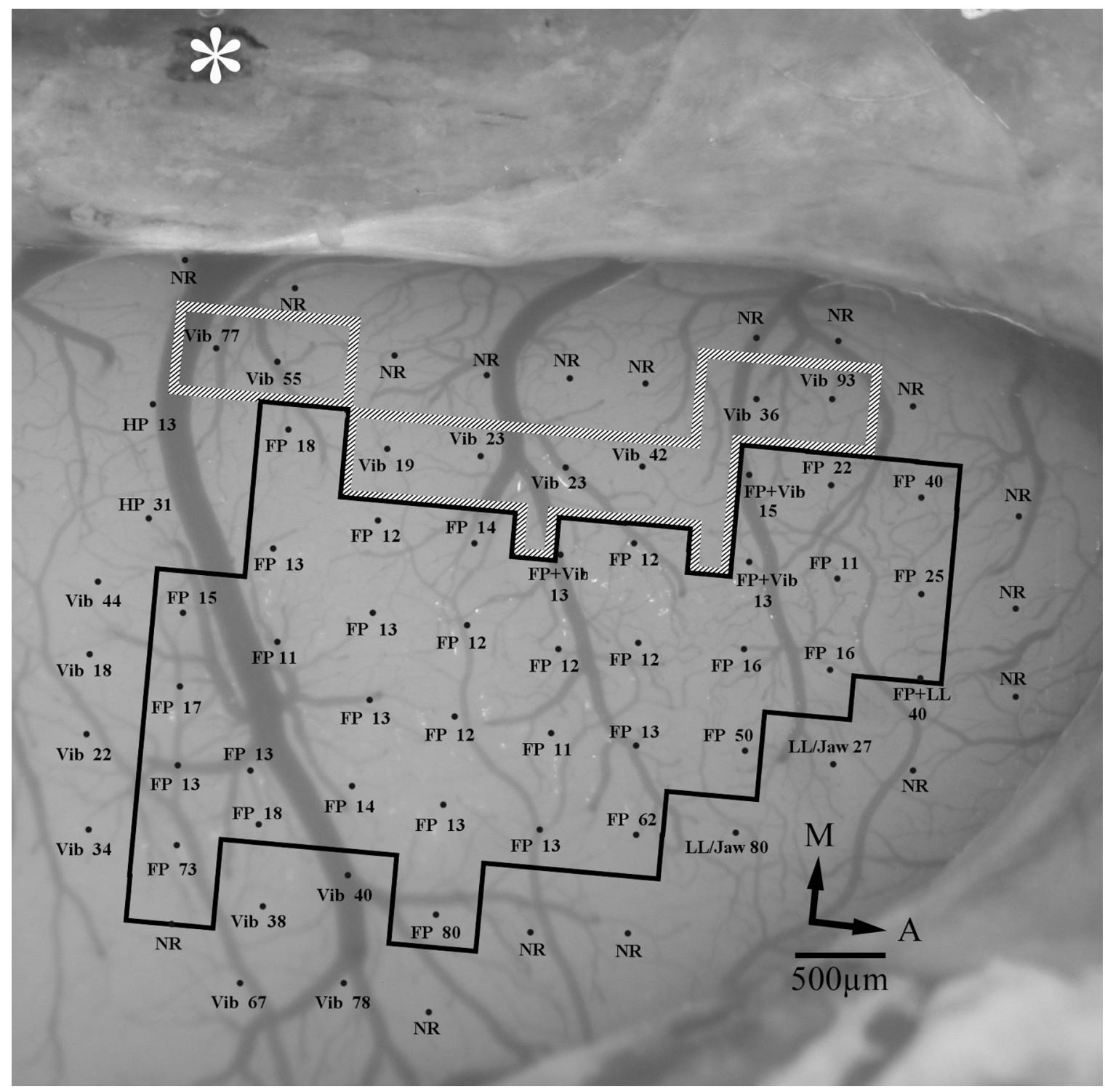

Figure 5.1 Forepaw and vibrissae motor map overlying cortical MI surface.

Diagonal hatched lines delineate the evoked vibrissae motor responses following ICMS. Dark solids lines indicate evoked forepaw motor responses following ICMS. Black dots indicate electrode penetration sites. Abbreviations indicate elicited motor response $\mathrm{FP}=$ forepaw, $\mathrm{Vib}=$ Vibrissae, $\mathrm{HP}=$ hindpaw, $\mathrm{LL}=$ lower-lip, Jaw $=$ jaw, $\mathrm{NR}=$ no response. Numeral following motor response indicates lowest evoked motor response in $\mu \mathrm{A}$. The large asterisk indicates bregma. Note, vibrissae and forepaw borders are raw data taken from the CF map in Fig 5.2A. However, here the grid is placed directly over the cortical image. 

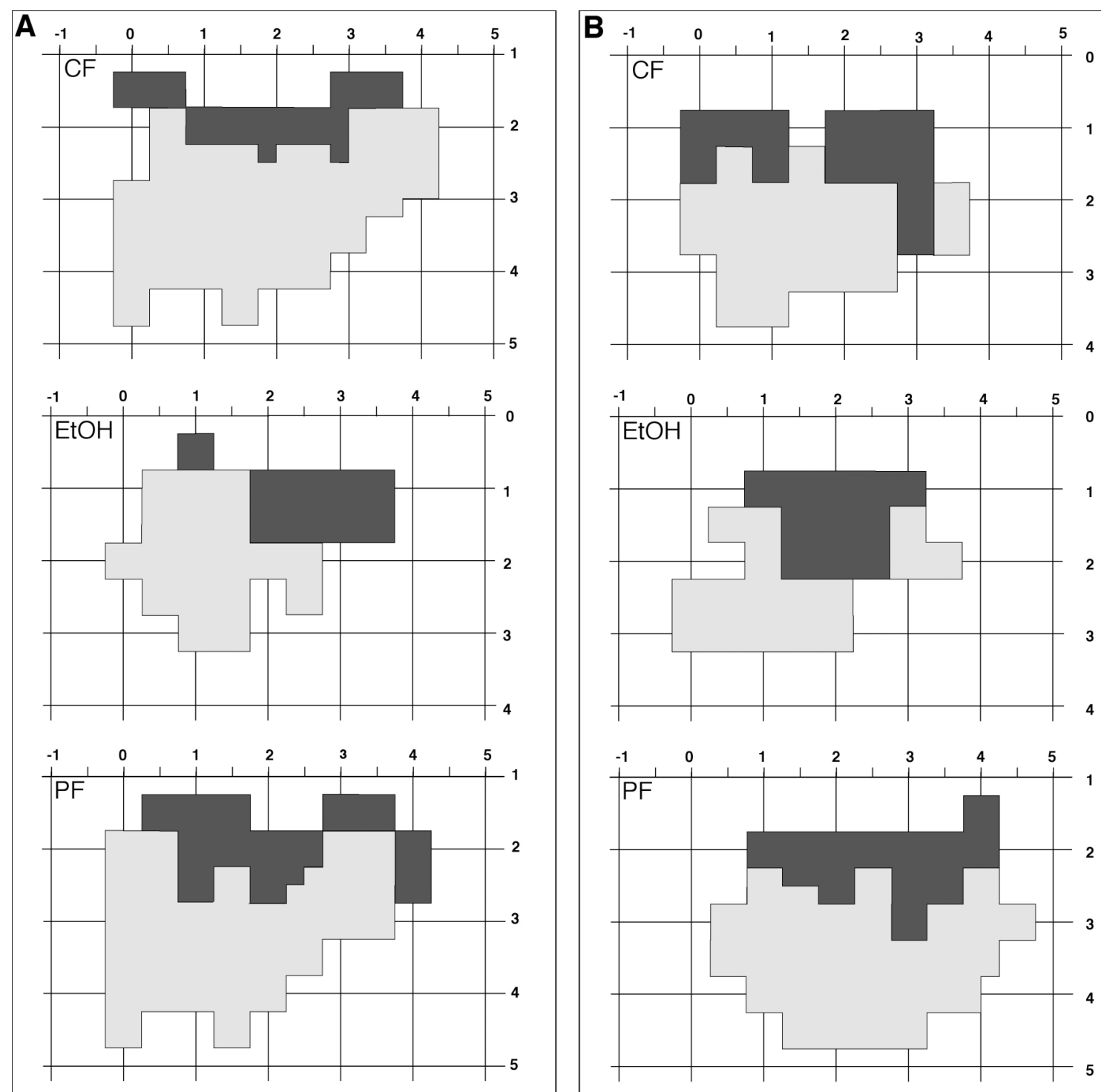

Figure 5.2 MI maps of forelimb and vibrissae evoked motor responses $\leq 100 \mu \mathrm{A}$.

Figure demonstrates typical vibrissae and forelimb motor maps with two examples for each group. Blackened regions indicate the area of the vibrissae motor representation in MI. Light gray regions indicate the areas of forelimb representation in MI. A) Motor map examples from each group. B) A second set of examples, one from each group. Notice that the motor responsive areas were similar in location between groups and only differed in overall area of motor cortex. " 0 " on the $y$-axis indicates bregma. All graphs are divided into $1 \mathrm{~mm} \times 1 \mathrm{~mm}$ grid plots. 
rostral direction. The forepaw representation was found more laterally where it bordered the vibrissae representation.

\subsubsection{Area of forepaw representation in MI}

Forepaw motor maps were constructed using stimulus currents $\leq 100 \mu \mathrm{A}$. There was a significant difference in the area of the forepaw representation in MI, between the three groups $[F(2,15)=4.317, p=0.033]$ and these results are shown in Table 5.1. The area of the motor representation for the EtOH group was significantly smaller than respective motor areas for the $\mathrm{CF}$ and $\mathrm{PF}$ groups. Data indicated that the area of forepaw representation in MI was $32.12 \pm 11.67 \%$ smaller than $\mathrm{CF}$ and $27.8 \pm 12.4 \%$ smaller than PF.

\subsubsection{Area of the vibrissae representation in MI}

The area of the vibrissae representations in MI was significantly different between the groups $[F(2,15)=3.969, p=0.0413]$. The area of the EtOH group was significantly reduced compared to respective areas in CF and PF groups (Table 5.1). Data indicated that the area of the vibrissae representation in the EtOH group was smaller by $29.45 \pm$ $11.82 \%$ compared to the $\mathrm{CF}$ group while EtOH was $30.3 \pm 11.7 \%$ smaller than the PF group.

\subsubsection{Combined areas of forepaw and vibrissae representations in MI}

There was a significant reduction in the combined areas of forepaw and vibrissae representations in MI $[\mathrm{F}(2,15)=11.548, \mathrm{p}<0.001]$ (Table 5.1). The combined area of 
Table 5.1 Forepaw and vibrissae motor map areas with thresholds $\leq 100 \mu \mathrm{A}$.

\begin{tabular}{lccc}
\hline Groups & EtOH $(n=6)$ & CF $(n=6)$ & PF $(n=6)$ \\
\hline Forepaw MI $\leq 100 \mu \mathrm{A}\left(\mathrm{mm}^{2}\right)$ & $5.14^{\mathrm{a}, \mathrm{b}}(0.88)$ & $7.58(0.50)$ & $7.13(0.36)$ \\
Vibrissae MI $\leq 100 \mu \mathrm{A}\left(\mathrm{mm}^{2}\right)$ & $2.00^{\mathrm{a}, \mathrm{b}}(0.35)$ & $2.96(0.24)$ & $2.99(0.14)$ \\
Forepaw and vibrissae $\mathrm{MI} \leq 100 \mu \mathrm{A}\left(\mathrm{mm}^{2}\right)$ & $7.23^{\mathrm{a}, \mathrm{b}}(0.74)$ & $10.53(0.39)$ & $10.05(0.39)$ \\
\hline
\end{tabular}

Data presented as the mean \pm SEM of forepaw and vibrissae MI areas that produced movements with ICMS thresholds $\leq 100 \mu \mathrm{A}$. A significant difference between groups indicated by: $\mathrm{EtOH}$ versus $\mathrm{CF}={ }^{\mathrm{a}}$, EtOH versus $\mathrm{PF}={ }^{\mathrm{b}}(\mathrm{P}<0.05)$. 
forepaw and vibrissae representations in MI, with thresholds $\leq 100 \mu \mathrm{A}$, was significantly reduced in the EtOH group compared to the respective area in the CF or PF groups. The EtOH group was $31.37 \pm 6.99 \%$ smaller than the $\mathrm{CF}$ group, while EtOH was $28.6 \pm 7.3 \%$ smaller than PF.

\subsubsection{The effect of PAE on ICMS thresholds for forepaw and vibrissae representation in $\mathrm{MI}$}

\subsubsection{ICMS thresholds in forepaw MI}

A significant difference in the mean threshold $(0-100 \mu \mathrm{A})$ required to produce a forepaw movement occurred between the groups $[F(2,15)=4.078, p=0.0385]$ (Table 5.2). The EtOH group had significantly higher thresholds than the CF, but not PF groups. The data indicated that the average threshold for EtOH was $33.2 \pm 5.5 \%$ higher than the CF group.

\subsubsection{ICMS thresholds in vibrissae MI}

No significant difference occurred between groups when the average ICMS threshold required to produce a vibrissae movement was analyzed (Table 5.2).

\subsubsection{Total ICMS thresholds for vibrissae and forepaw representation in MI}

There was a significant difference in the thresholds for all vibrissae and forepaw $[\mathrm{F}(2,15)=3.976, \mathrm{p}=0.0412]$ (Table 5.2). The average threshold for the EtOH group was higher than it was for the CF group, but not the PF group. the data showed that the 
Table 5.2 Average threshold $(\leq 100 \mu \mathrm{A})$ needed to produce motor maps.

\begin{tabular}{lccc}
\hline Groups & EtOH $(n=6)$ & CF $(n=6)$ & PF $(n=6)$ \\
\hline Average forepaw threshold $\leq 100(\mu \mathrm{A})$ & $47.0^{\mathrm{a}}(5.9)$ & $31.4(2.6)$ & $38.0(2.0)$ \\
Average vibrissae threshold $\leq 100(\mu \mathrm{A})$ & $50.2(4.7)$ & $42.3(2.5)$ & $43.8(3.5)$ \\
$\begin{array}{l}\text { Average forepaw + vibrissae thresholds } \\
\leq 100(\mu \mathrm{A})\end{array}$ & $47.0^{\mathrm{a}}(4.1)$ & $35.1(2.4)$ & $40.1(2.1)$ \\
\hline
\end{tabular}

Data presented as mean \pm SEM of thresholds required to produce forepaw and vibrissae motor maps. A significant difference between groups indicated by: $\mathrm{EtOH}$ versus $\mathrm{CF}={ }^{\mathrm{a}}$ $(\mathrm{P}<0.05)$. 
average threshold for the combined vibrissae and forepaw MI was $25.29 \pm 5.12 \%$ higher in the EtOH group than in the CF group.

\subsection{Discussion}

Previous studies in humans with FASD have resulted in the identification of numerous motor-related deficits in: balance (Roebuck, et al., 1998b), posture (Roebuck, et al., 1998a), cognitive-motor development such as hand-eye coordination (Adnams, et al., 2001), motor coordination (Bookstein, et al., 2002b), fine motor (Connor, et al., 2006; Janzen, Nanson \& Block, 1995) and spatial fine motor control (Kaplan-Estrin, Jacobson \& Jacobson, 1999). In addition, studies on PAE rodent models showed deficits in motorrelated behavioral tasks similar to those seen in children with FASD (Goodlett \& Lundahl, 1996; Norton, et al., 1988; Thomas, et al., 1996). Animal models have also been used to identify motor-related brain regions affected by PAE (Inomata, Nasu \& Tanaka, 1987; Popova, 1989). To this end, we hypothesized that PAE might also affect MI by reducing the area of cortex representing vibrissae and forelimb movement as well as increasing the threshold of ICMS required to elicit a motor movement. The major findings of this study are that PAE significantly: 1) reduced the area of the forepaw motor map; 2) reduced the area of the vibrissae motor map; and 3) reduced the combined area of the forepaw and vibrissae motor map.

While technical limitations (such as current spread, threshold determination, and anesthesia level for each animal during mapping) are inherent when mapping the motor cortex with ICMS, our procedures are similar to reported motor mapping findings in rat (Donoghue \& Wise, 1982; Gioanni \& Lamarche, 1985; Hall \& Lindholm, 1974; 
Miyashita, Keller \& Asanuma, 1994; Neafsey, et al., 1986; Neafsey \& Sievert, 1982) and mouse (Li \& Waters, 1991). Similar to the present study, previous investigators used stimulating currents $\leq 100 \mu \mathrm{A}$ to map the motor cortex (Asanuma, Arnold \& Zarzecki, 1976; Bagshaw \& Evans, 1976; Neafsey, et al., 1986; Ranck, 1975). Results from these studies indicated that an ICMS current of $100 \mu \mathrm{A}$ spreads $\sim 250 \mu \mathrm{m}$ from the electrode. As $250 \mu \mathrm{m}$ is approximately half the distance between two adjacent electrode penetrations in this study, the maximum ICMS current of $100 \mu \mathrm{A}$ would not overlap the midpoint distance between two proximal electrode penetrations. To produce MI maps in this study, movements elicited at the lowest threshold were used as data points to represent a penetration. However when the representation of two motor movements (i.e., one forepaw and one vibrissae) were identified at the same threshold within a single penetration, both movements were designated as a data point representing those penetrations, see Neafsey et al. (1986). While the boundaries of responsive MI are arbitrary in that higher ICMS current can produce movement, only movements elicited at thresholds $\leq 100 \mu \mathrm{A}$ were noted. Reliance on our threshold data reduced concerns associated with using higher ICMS currents that can spread, producing complex movements (Hall \& Lindholm, 1974). Motor responses elicited by ICMS were dependent on the level of anesthesia during mapping. To control for anesthesia levels, animals were provided supplemental doses of ketamine/xylazine when spontaneous vibrissae and/or forepaw movements were observed (see Brecht et al 2004a). By limiting the ICMS current to $\leq 100 \mu \mathrm{A}$ and maintaining rats in a light state of areflexia, motor maps were similar to the maps in previous studies (Brecht, et al., 2004b; Donoghue \& Wise, 1982; Li \& Waters, 1991). 
The forepaw and vibrissae representations in the MI maps in this study (PAE and non-PAE alike) were similar to the maps in previous studies (Donoghue \& Wise, 1982; Gioanni \& Lamarche, 1985; Hall \& Lindholm, 1974; Li \& Waters, 1991; Miyashita, Keller \& Asanuma, 1994; Neafsey, et al., 1986; Neafsey \& Sievert, 1982). In brief, a narrow vibrissae representation was typically found lying medial to a much larger area of MI representing the forepaw. Infrequently, a second vibrissae area was identified lying posterior and lateral to the medial strip of the vibrissae responsive zone; however, a hindlimb responsive zone or a non-responsive zone always separated these two vibrissae representations in MI. Only the medial vibrissae area was measured in this study because the posterior lateral vibrissae may be part of a much larger region of primary somatosensory cortex extending into granular cortex (Neafsey, et al., 1986). Unresponsive regions were found anterior to forepaw and vibrissae regions, as well as lateral to the forepaw.

It has been revealed in many motor studies that PAE affects layer $\mathrm{V}$ of cortex. When acute PAE of $18 \mathrm{~g} / \mathrm{kg}$ of alcohol was given to pregnant rats on GD14 and GD15, their offspring showed significant delays in motor activity and speed of movements with a concomitant decrease in thickness of layer $\mathrm{V}$ of cerebral cortex as well as a decrease in total cortical thickness at PD28 (Kotkoskie \& Norton, 1989). Similarly, exposure to alcohol between GD9 to GD12 significantly reduced layer V cortical thickness with a concomitant 44\% decrease in brain weight at PD42 (Fakoya \& Caxton-Martins, 2006). This effect of prenatal alcohol was so devastating on layer $\mathrm{V}$ as to produce many pyknotic pyramidal neurons that consisted of many broken dendrites, and collapsed cell bodies with obliterated nuclei and nucleoli. Furthermore, normal cell packing density in 
layer V pyramidal neurons was decreased by 55\%. However, Fakoya and Caxton-Martins (2006) stated that the overall cortical thickness was not changed in EtOH rats due to a concomitant increase in layer II/III, apparently matching the decrease in layer $\mathrm{V}$ thickness. In a similar study, dendritic spines of SI pyramidal neurons in layer V were significantly reduced, but appeared long and thin only at PD15 (Galofre, et al., 1987). However, these changes were not apparent at PD30 and PD90. The improvements identified at PD30 and PD90 contradict the persistent deficits that occurred until PD42 in the study by Fakoya and Caxton-Martins (2006). Electron microscopic examination of layer V cortex in EtOH treated rats resulted in: cisternae that were less organized; Golgi complex and lysosomes that occupied more of the somata while symmetric axosomatic synapses (but not asymmetric axosomatic synapses) occurred more often in local circuit neurons; and dendritic shafts that occupied less space while axons filled more of the neuropil (al-Rabiai \& Miller, 1989). The increase in axonal space was apparently due to a significant increase in non-myelinated axons and significantly fewer myelinated axons (al-Rabiai \& Miller, 1989). A similar cortical ultrastructure also has been shown in other studies (Popova, 1989).

Intracellular cell records in an in vitro slice preparation, bathed in physiological relevant concentrations of ethanol, resulted in reduced excitability of layer $\mathrm{V}$ and layer II/III cortical neurons by increasing the rheobase (electrical current threshold), decreased input resistance and repetitive firing, and reduced postsynaptic potentials amplitude and the probability of evoking action potentials (Sessler, et al., 1998). This study is extremely relevant to the current chapter as each of the Sessler et al. (1998) results are directly related to the higher thresholds needed to facilitate motor movement (between EtOH and 
CF groups only), which in turn may decrease the area of the MI representative map in the EtOH group with thresholds of $\leq 100 \mu \mathrm{A}$. This is due to the possibility that additional motor movements may have been produced in the edges of maps that bordered nonresponsive zones, if the $100 \mu \mathrm{A}$ limit had not been set in the present study. An additional study has revealed that PAE cortical deficits were not confined to layer V as synaptic density was decreased in molecular Layer I of the motor cortex following PAE (Inomata, Nasu \& Tanaka, 1987).

The results of our motor cortex examination lend support to previous animal behavioral studies that demonstrated the significant affects of PAE on motor performance: Chronic PAE from GD5 to PD10 resulted in rats that were significantly delayed by two days in a reflex suspension task (the time an animal maintained grip on a crossbar) and significantly slower negative geotaxis or the time they took to rotate 180 degrees compared to controls (Norton, et al., 1988). PAE produced hyperactivity in PD10 guinea pigs (Gibson, et al., 2000). Alcohol exposure during third trimester human equivalent also produced significant impairments on the parallel bar task in PD42-44 rats (Goodlett \& Lundahl, 1996; Thomas, et al., 1996). However, the effect seemingly disappeared by PD80, as adult alcohol rats were indistinguishable from controls in openfield activity behaviors (Melcer, Gonzalez \& Riley, 1995). Furthermore, PAE produced an altered gait in rats, and a stride similar to that of an ataxic animal (Hannigan \& Riley, 1988; Melcer, et al., 1995). While an abnormal gait persisted in rats as old as PD87

(Meyer, Kotch \& Riley, 1990), environmental enrichment ameliorated this condition and improved the stride of EtOH rats until their gait appeared equal to controls (Hannigan, Berman \& Zajac, 1993). 
In light of our results, the effect of PAE on vibrissae MI area may seriously disrupt rodent perception of its environment, as vibrissae movement is tuned to resonate at specific frequencies to assist in sensory perception (Andermann, et al., 2004). A combination of less MI cortical area devoted to vibrissae movement, and the slightly increased ICMS threshold levels required to produce movement may distort the processing capabilities of a rat exposed to alcohol during gestation.

\subsection{Conclusion}

In summary, the current chapter indicated that PAE significantly reduced the motor cortex area devoted to vibrissae and forepaw movements with thresholds $\leq 100 \mu \mathrm{A}$, while the effect on ICMS threshold for forepaw and vibrissae movements may be more dependent upon nutrition then PAE. These findings suggest that the effect of PAE on MI persists in juvenile rats and may provide an explanation for some of the deficits related to balance, posture, and fine motor movement in children with FASD. Results from the current and previous studies provide evidence that PAE significantly disrupts the morphological and physiological characteristics of sensorimotor cortex and also produces long-term deficits in behaviors associated with the functions of the sensorimotor system. 


\section{LIST OF REFERENCES}

Abel, E.L. (1984a). Prenatal effects of alcohol. Drug Alcohol Depend, 14(1), 1-10. Abel, E.L. (1984b). Smoking and pregnancy. J Psychoactive Drugs, 16(4), 327-38. Abel, E.L. (1998). Fetal Alcohol Abuse Syndrome. New York: Plenum Press.

Abel, E.L. (1999). Was the fetal alcohol syndrome recognized by the Greeks and Romans? Alcohol and Alcoholism, 34(6), 868-72.

Abel, E.L., \& Hannigan, J.H. (1995). Maternal risk factors in fetal alcohol syndrome: provocative and permissive influences. Neurotoxicol Teratol, 17(4), 445-62.

Abel, E.L., \& Sokol, R.J. (1987). Incidence of fetal alcohol syndrome and economic impact of FAS-related anomalies. Drug Alcohol Depend, 19(1), 51-70.

Abel, E.L., \& Sokol, R.J. (1991). A revised conservative estimate of the incidence of FAS and its economic impact. Alcohol Clin Exp Res, 15(3), 514-24.

Adnams, C.M., Kodituwakku, P.W., Hay, A., Molteno, C.D., Viljoen, D., \& May, P.A. (2001). Patterns of cognitive-motor development in children with fetal alcohol syndrome from a community in South Africa. Alcohol Clin Exp Res, 25(4), 55762.

Ahissar, E., \& Arieli, A. (2001). Figuring space by time. Neuron, 32(2), 185-201.

Ahissar, E., Sosnik, R., \& Haidarliu, S. (2000). Transformation from temporal to rate coding in a somatosensory thalamocortical pathway. Nature, 406(6793), 302-6.

al-Rabiai, S., \& Miller, M.W. (1989). Effect of prenatal exposure to ethanol on the ultrastructure of layer V of mature rat somatosensory cortex. J Neurocytol, 18(6), 711-29.

Allan, A.M., Wu, H., Paxton, L.L., \& Savage, D.D. (1998). Prenatal ethanol exposure alters the modulation of the gamma-aminobutyric acidA1 receptor-gated chloride ion channel in adult rat offspring. J Pharmacol Exp Ther, 284(1), $250-7$.

Ames, B.N., \& Saul, R.L. (1986). Oxidative DNA damage as related to cancer and aging. Prog Clin Biol Res, 209A, 11-26.

Andermann, M.L., Ritt, J., Neimark, M.A., \& Moore, C.I. (2004). Neural correlates of vibrissa resonance; band-pass and somatotopic representation of high-frequency stimuli. Neuron, 42(3), 451-63.

Anderson, S.A., Marin, O., Horn, C., Jennings, K., \& Rubenstein, J.L. (2001). Distinct cortical migrations from the medial and lateral ganglionic eminences.

Development, 128(3), 353-63.

Angelucci, F., Cimino, M., Balduini, W., Piltillo, L., \& Aloe, L. (1997). Prenatal exposure to ethanol causes differential effects in nerve growth factor and its receptor in the basal forebrain of preweaning and adult rats. J Neural Transplant Plast, 6(2), 63-71.

Archibald, S.L., Fennema-Notestine, C., Gamst, A., Riley, E.P., Mattson, S.N., \& Jernigan, T.L. (2001). Brain dysmorphology in individuals with severe prenatal alcohol exposure. Dev Med Child Neurol, 43(3), 148-54.

Arendt, T. (1994). Impairment in memory function and neurodegenerative changes in the cholinergic basal forebrain system induced by chronic intake of ethanol. J Neural Transm Suppl, 44, 173-87. 
Armstrong-James, M., \& Fox, K. (1987). Spatio-temporal divergence and convergence in rat "barrel" cortex. J Comp Neurol, 263, 265-281.

Armstrong-James, M., Fox, K., \& Das- Gupta, A. (1992). Flow of excitation within barrel cortex on striking a single vibrissa. J Neurophysiol, 68, 1345-1358.

Arnold, P.B., Li, C.X., \& Waters, R.S. (2001). Thalamocortical arbors extend beyond single cortical barrels: an in vivo tracing study in the rat. Exp Brain Res, 136, 152168.

Asanuma, H., Arnold, A., \& Zarzecki, P. (1976). Further study on the excitation of pyramidal tract cells by intracortical microstimulation. Exp Brain Res, 26(5), 44361.

Asanuma, H., \& Rosen, I. (1972a). Functional role of afferent inputs to the monkey motor cortex. Brain Res, 40(1), 3-5.

Asanuma, H., \& Rosen, I. (1972b). Topographical organization of cortical efferent zones projecting to distal forelimb muscles in the monkey. Exp Brain Res, 14(3), 24356.

Ashwell, K.W., \& Zhang, L.L. (1996). Forebrain hypoplasia following acute prenatal ethanol exposure: quantitative analysis of effects on specific forebrain nuclei. Pathology, 28(2), 161-6.

Bagshaw, E.V., \& Evans, M.H. (1976). Measurement of current spread from microelectrodes when stimulating within the nervous system. Exp Brain Res, 25(4), 391-400.

Bailey, B.N., Delaney-Black, V., Covington, C.Y., Ager, J., Janisse, J., Hannigan, J.H., \& Sokol, R.J. (2004). Prenatal exposure to binge drinking and cognitive and behavioral outcomes at age 7 years. Am J Obstet Gynecol, 191(3), 1037-43.

Bailey, C.D., Brien, J.F., \& Reynolds, J.N. (2004). Chronic prenatal ethanol exposure alters the proportion of GABAergic neurons in layers II/III of the adult guinea pig somatosensory cortex. Neurotoxicol Teratol, 26(1), 59-63.

Bailey, S.M., Pietsch, E.C., \& Cunningham, C.C. (1999). Ethanol stimulates the production of reactive oxygen species at mitochondrial complexes I and III. Free Radic Biol Med, 27(7-8), 891-900.

Barnes, D.E., \& Walker, D.W. (1981). Prenatal ethanol exposure permanently reduces the number of pyramidal neurons in rat hippocampus. Brain Res, 227(3), 333-40.

Baruah, J.K., \& Kinder, D. (1989). Pathological changes in peripheral nerves in experimental fetal alcohol syndrome. Alcohol Clin Exp Res, 13(4), 547-8.

Bennett, E.L., Rosenzweig, M.R., Diamond, M.C., Morimoto, H., \& Hebert, M. (1974). Effects of successive environments on brain measures. Physiol Behav, 12(4), 62131.

Bernardo, K.L., McCasland, J.S., \& Woolsey, T.A. (1990). Local axonal trajectories in mouse barrel cortex. Exp Brain Res, 82(2), 247-53.

Bernardo, K.L., McCasland, J.S., Woolsey, T.A., \& Strominger, R.N. (1990). Local intraand interlaminar connections in mouse barrel cortex. J Comp Neurol, 291(2), 23155.

Bhave, S.V., \& Hoffman, P.L. (1997). Ethanol promotes apoptosis in cerebellar granule cells by inhibiting the trophic effect of NMDA. J Neurochem, 68(2), 578-86. 
Bingham, S.M., Mudd, L.M., Lopez, T.F., \& Montague, J.R. (2004). Effects of ethanol on cultured embryonic neurons from the cerebral cortex of the rat. Alcohol, 32(2), 129-35.

Black, J.E., Zelazny, A.M., \& Greenough, W.T. (1991). Capillary and mitochondrial support of neural plasticity in adult rat visual cortex. Exp Neurol, 111(2), 204-9.

Bonthius, D.J., Goodlett, C.R., \& West, J.R. (1988). Blood alcohol concentration and severity of microencephaly in neonatal rats depend on the pattern of alcohol administration. Alcohol, 5(3), 209-14.

Bonthius, D.J., \& West, J.R. (1988). Blood alcohol concentration and microencephaly: a dose-response study in the neonatal rat. Teratology, 37(3), 223-31.

Bookstein, F.L., Sampson, P.D., Connor, P.D., \& Streissguth, A.P. (2002a). Midline corpus callosum is a neuroanatomical focus of fetal alcohol damage. Anat Rec, 269(3), 162-74.

Bookstein, F.L., Sampson, P.D., Streissguth, A.P., \& Connor, P.D. (2001). Geometric morphometrics of corpus callosum and subcortical structures in the fetal-alcoholaffected brain. Teratology, 64(1), 4-32.

Bookstein, F.L., Streissguth, A.P., Sampson, P.D., Connor, P.D., \& Barr, H.M. (2002b). Corpus callosum shape and neuropsychological deficits in adult males with heavy fetal alcohol exposure. Neuroimage, 15(1), 233-51.

Boylan, C.B., Kesterson, K.L., Bennett-Clarke, C.A., Chiaia, N.L., \& Rhoades, R.W. (2001). Neither peripheral nerve input nor cortical NMDA receptor activity are necessary for recovery of a disrupted barrel pattern in rat somatosensory cortex. Brain Res Dev Brain Res, 129(1), 95-106.

Brecht, M., Krauss, A., Muhammad, S., Sinai-Esfahani, L., Bellanca, S., \& Margrie, T.W. (2004a). Organization of rat vibrissa motor cortex and adjacent areas according to cytoarchitectonics, microstimulation, and intracellular stimulation of identified cells. J Comp Neurol, 479(4), 360-73.

Brecht, M., Preilowski, B., \& Merzenich, M.M. (1997). Functional architecture of the mystacial vibrissae. Behav Brain Res, 84(1-2), 81-97.

Brecht, M., Schneider, M., Sakmann, B., \& Margrie, T.W. (2004b). Whisker movements evoked by stimulation of single pyramidal cells in rat motor cortex. Nature, 427(6976), 704-10.

Bronchti, G., Corthesy, M.E., \& Welker, E. (1999). Partial denervation of the whiskerpad in adult mice: altered patterns of metabolic activity in barrel cortex. Eur $J$ Neurosci, 11(8), 2847-55.

Brown, R.T., Coles, C.D., Smith, I.E., Platzman, K.A., Silverstein, J., Erickson, S., \& Falek, A. (1991). Effects of prenatal alcohol exposure at school age. II. Attention and behavior. Neurotoxicol Teratol, 13(4), 369-76.

Brumberg, J.C., Pinto, D.J., \& Simons, D.J. (1996). Spatial gradients and inhibitory summation in the rat whisker barrel system. J Neurophysiol, 76(1), 130-40.

Burd, L., Cotsonas-Hassler, T.M., Martsolf, J.T., \& Kerbeshian, J. (2003a). Recognition and management of fetal alcohol syndrome. Neurotoxicol Teratol, 25(6), 681-8.

Burd, L., Klug, M.G., Martsolf, J.T., \& Kerbeshian, J. (2003b). Fetal alcohol syndrome: neuropsychiatric phenomics. Neurotoxicol Teratol, 25(6), 697-705. 
Burden, M.J., Jacobson, S.W., \& Jacobson, J.L. (2005). Relation of prenatal alcohol exposure to cognitive processing speed and efficiency in childhood. Alcohol Clin Exp Res, 29(8), 1473-83.

Cartwright, M.M., \& Smith, S.M. (1995). Increased cell death and reduced neural crest cell numbers in ethanol-exposed embryos: partial basis for the fetal alcohol syndrome phenotype. Alcohol Clin Exp Res, 19(2), 378-86.

Carvell, G.E., \& Simons, D.J. (1990). Biometric analyses of vibrissal tactile discrimination in the rat. $J$ Neurosci, 10(8), 2638-48.

Catalano, S.M., Robertson, R.T., \& Killackey, H.P. (1996). Individual axon morphology and thalamocortical topography in developing rat somatosensory cortex. J Comp Neurol, 367(1), 36-53.

Chan, W.Y., Lorke, D.E., Tiu, S.C., \& Yew, D.T. (2002). Proliferation and apoptosis in the developing human neocortex. Anat Rec, 267(4), 261-76.

Chapin, J.K., \& Lin, C.S. (1984). Mapping the body representation in the SI cortex of anesthetized and awake rats. J Comp Neurol, 229(2), 199-213.

Chapin, J.K., Sorensen, S.M., \& Woodward, D.J. (1986). Acute ethanol effects on sensory responses of single units in the somatosensory cortex of rats during different behavioral states. Pharmacol Biochem Behav, 25(3), 607-14.

Chapin, J.K., \& Woodward, D.J. (1989). Ethanol withdrawal increases sensory responsiveness of single somatosensory cortical neurons in the awake, behaving rat. Alcohol Clin Exp Res, 13(1), 8-14.

Chappell, T.D., Oladehin, A., Margret, C.P., Li, C.X., Elberger, A.J., \& Waters, R.S. (2005). Prenatal alcohol exposure (PAE) reduces cell number in whisker barrel cortex in rats examined at six weeks of age: A Neu-N labeling study. Alcohol Clin Exp Res, 29, 88-A.

Chaudhuri, J.D. (2000). Alcohol and the developing fetus--a review. Med Sci Monit, 6(5), 1031-41.

Chen, S.Y., Dehart, D.B., \& Sulik, K.K. (2004). Protection from ethanol-induced limb malformations by the superoxide dismutase/catalase mimetic, EUK-134. Faseb J, 18(11), 1234-6.

Chiriboga, C.A. (2003). Fetal alcohol and drug effects. Neurologist, 9(6), 267-79.

Chmielowska, J., Carvell, G.E., \& Simons, D.J. (1989). Spatial organization of thalamocortical and corticothalamic projection systems in the rat SmI barrel cortex. J Comp Neurol, 285(3), 325-38.

Choi, D.W. (1995). Calcium: still center-stage in hypoxic-ischemic neuronal death. Trends Neurosci, 18(2), 58-60.

Chrisman, K., Kenney, R., Comin, J., Thal, T., Suchocki, L., Yueh, Y.G., \& Gardner, D.P. (2004). Gestational ethanol exposure disrupts the expression of FGF8 and Sonic hedgehog during limb patterning. Birth Defects Res A Clin Mol Teratol, 70(4), 163-71.

Church, M.W. (1987). Chronic in utero alcohol exposure affects auditory function in rats and in humans. Alcohol, 4(4), 231-9.

Church, M.W., \& Abel, E.L. (1998). Fetal alcohol syndrome. Hearing, speech, language, and vestibular disorders. Obstet Gynecol Clin North Am, 25(1), 85-97. 
Church, M.W., Eldis, F., Blakley, B.W., \& Bawle, E.V. (1997). Hearing, language, speech, vestibular, and dentofacial disorders in fetal alcohol syndrome. Alcohol Clin Exp Res, 21(2), 227-37.

Church, M.W., Jen, K.L., Pellizzon, M.A., \& Holmes, P.A. (1998). Prenatal cocaine, alcohol, and undernutrition differentially alter mineral and protein content in fetal rats. Pharmacol Biochem Behav, 59(3), 577-84.

Churchill, J.D., Galvez, R., Colcombe, S., Swain, R.A., Kramer, A.F., \& Greenough, W.T. (2002). Exercise, experience and the aging brain. Neurobiol Aging, 23(5), 941-55.

Clarren, S.K., Alvord, E.C., Jr., Sumi, S.M., Streissguth, A.P., \& Smith, D.W. (1978). Brain malformations related to prenatal exposure to ethanol. J Pediatr, 92(1), 647.

Clarren, S.K., Astley, S.J., \& Bowden, D.M. (1988). Physical anomalies and developmental delays in nonhuman primate infants exposed to weekly doses of ethanol during gestation. Teratology, 37(6), 561-9.

Coles, C.D., Brown, R.T., Smith, I.E., Platzman, K.A., Erickson, S., \& Falek, A. (1991). Effects of prenatal alcohol exposure at school age. I. Physical and cognitive development. Neurotoxicol Teratol, 13(4), 357-67.

Connor, P.D., Sampson, P.D., Bookstein, F.L., Barr, H.M., \& Streissguth, A.P. (2000). Direct and indirect effects of prenatal alcohol damage on executive function. Dev Neuropsychol, 18(3), 331-54.

Connor, P.D., Sampson, P.D., Streissguth, A.P., Bookstein, F.L., \& Barr, H.M. (2006). Effects of prenatal alcohol exposure on fine motor coordination and balance: A study of two adult samples. Neuropsychologia, 44(5), 744-51.

Connor, P.D., Streissguth, A.P., Sampson, P.D., Bookstein, F.L., \& Barr, H.M. (1999). Individual differences in auditory and visual attention among fetal alcoholaffected adults. Alcohol Clin Exp Res, 23(8), 1395-402.

Conry, J. (1990). Neuropsychological deficits in fetal alcohol syndrome and fetal alcohol effects. Alcohol Clin Exp Res, 14(5), 650-5.

Coq, J.O., \& Xerri, C. (1998). Environmental enrichment alters organizational features of the forepaw representation in the primary somatosensory cortex of adult rats. Exp Brain Res, 121(2), 191-204.

Coq, J.O., \& Xerri, C. (1999). Tactile impoverishment and sensorimotor restriction deteriorate the forepaw cutaneous map in the primary somatosensory cortex of adult rats. Exp Brain Res, 129(4), 518-31.

Coq, J.O., \& Xerri, C. (2000). Age-related alteration of the forepaw representation in the rat primary somatosensory cortex. Neuroscience, 99(3), 403-11.

Corthesy, M.E., Bronchti, G., \& Welker, E. (1999). Partial denervation of the whiskerpad in adult mice: pattern and origin of reinnervation. Eur J Neurosci, 11(8), 2835-46.

Couch, D.B., \& Baker, R.C. (2002). Ethanol-enhanced cytotoxicity of alkylating agents. Alcohol Clin Exp Res, 26(3), 381-5.

Covington, C.Y., Nordstrom-Klee, B., Ager, J., Sokol, R., \& Delaney-Black, V. (2002). Birth to age 7 growth of children prenatally exposed to drugs: a prospective cohort study. Neurotoxicol Teratol, 24(4), 489-96. 
Crawford, M.L., Meharg, L.S., \& Johnston, D.A. (1982). Structure of columns in monkey striate cortex induced by luminant-contrast and color-contrast stimulation. Proc Natl Acad Sci U S A, 79(21), 6722-6.

Davis, R.L., \& Syapin, P.J. (2004). Acute ethanol exposure modulates expression of inducible nitric-oxide synthase in human astroglia: evidence for a transcriptional mechanism. Alcohol, 32(3), 195-202.

Day, N.L., Leech, S.L., Richardson, G.A., Cornelius, M.D., Robles, N., \& Larkby, C. (2002). Prenatal alcohol exposure predicts continued deficits in offspring size at 14 years of age. Alcohol Clin Exp Res, 26(10), 1584-91.

Diamond, M.C., Johnson, R.E., Ingham, C., Rosenzweig, M.R., \& Bennett, E.L. (1975). Effects of differential experience on neuronal nuclear and perikarya dimensions in the rat cerebral cortex. Behav Biol, 15(1), 107-11.

Diamond, M.E. (1995). Somatosensory thalamus of the rat, The Barrel Cortex of Rodents. 189-219. New York: Plenum Press.

Diamond, M.E., Armstrong-James, M., Budway, M.J., \& Ebner, F.F. (1992). Somatic sensory responses in the rostral sector of the posterior group (POm) and in the ventral posterior medial nucleus (VPM) of the rat thalamus: dependence on the barrel field cortex. J Comp Neurol, 319(1), 66-84.

Diamond, M.E., Armstrong-James, M., \& Ebner, F.F. (1992). Somatic sensory responses in the rostral sector of the posterior group (POm) and in the ventral posterior medial nucleus (VPM) of the rat thalamus. J Comp Neurol, 318(4), 462-76.

Diamond, M.E., Petersen, R.S., Harris, J.A., \& Panzeri, S. (2003). Investigations into the organization of information in sensory cortex. J Physiol Paris, 97(4-6), 529-36.

Dobbing, J., \& Sands, J. (1973). Quantitative growth and development of human brain. Arch Dis Child, 48(10), 757-67.

Dobbing, J., \& Sands, J. (1979). Comparative aspects of the brain growth spurt. Early Hum Dev, 3(1), 79-83.

Donoghue, J.P., \& Sanes, J.N. (1994). Motor areas of the cerebral cortex. J Clin Neurophysiol, 11(4), 382-96.

Donoghue, J.P., \& Wise, S.P. (1982). The motor cortex of the rat: cytoarchitecture and microstimulation mapping. J Comp Neurol, 212(1), 76-88.

Duester, G. (1991). A hypothetical mechanism for fetal alcohol syndrome involving ethanol inhibition of retinoic acid synthesis at the alcohol dehydrogenase step. Alcohol Clin Exp Res, 15(3), 568-72.

Dunty, W.C., Jr., Zucker, R.M., \& Sulik, K.K. (2002). Hindbrain and cranial nerve dysmorphogenesis result from acute maternal ethanol administration. Dev Neurosci, 24(4), 328-42.

Durham, D., \& Woolsey, T.A. (1977). Barrels and columnar cortical organization: evidence from 2-deoxyglucose (2-DG) experiments. Brain Res, 137(1), 168-74.

Durham, D., \& Woolsey, T.A. (1985). Functional organization in cortical barrels of normal and vibrissae-damaged mice: a $(3 \mathrm{H}) 2$-deoxyglucose study. J Comp Neurol, 235(1), 97-110.

Ebner, F.F., \& Armstrong-James, M.A. (1990). Intracortical processes regulating the integration of sensory information. Prog Brain Res, 86, 129-41. 
Erzurumlu, R.S., \& Jhaveri, S. (1990). Thalamic axons confer a blueprint of the sensory periphery onto the developing rat somatosensory cortex. Brain Res Dev Brain Res, 56(2), 229-34.

Fadem, B.H., Tassava, R.A., Miller, S.I., \& Congleton, L.A. (1989). Limb defects in gray short-tailed opossums (Monodelphis domestica) following postnatal injection with ethanol or saline. Teratog Carcinog Mutagen, 9(1), 1-6.

Fakoya, F. (2004). Persistant neocortical astrogliosis in adult wistar rats following prenatal ethanol exposure. Brain and Development, 1-7.

Fakoya, F.A. (2005). Persistent neocortical astrogliosis in adult wistar rats following prenatal ethanol exposure. Brain Dev, 27(4), 259-65.

Fakoya, F.A., \& Caxton-Martins, E.A. (2006). Neocortical neurodegeneration in young adult Wistar rats prenatally exposed to ethanol. Neurotoxicol Teratol, 28(2), 22937.

Falconer, J. (1990). The effect of maternal ethanol infusion on placental blood flow and fetal glucose metabolism in sheep. Alcohol and Alcoholism, 25(4), 413-6.

Famy, C., Streissguth, A.P., \& Unis, A.S. (1998). Mental illness in adults with fetal alcohol syndrome or fetal alcohol effects. Am J Psychiatry, 155(4), 552-4.

Favorov, O.V., \& Diamond, M.E. (1990). Demonstration of discrete place-defined columns--segregates--in the cat SI. J Comp Neurol, 298(1), 97-112.

Ferchmin, P.A., \& Eterovic, V.A. (1986). Forty minutes of experience increase the weight and RNA content of cerebral cortex in periadolescent rats. Dev Psychobiol, 19(6), 511-9.

Ferchmin, P.A., \& Eterovic, V.A. (1987). Role of polyamines in experience-dependent brain plasticity. Pharmacol Biochem Behav, 26(2), 341-9.

Ferrer, I., \& Galofre, E. (1987). Dendritic spine anomalies in fetal alcohol syndrome. Neuropediatrics, 18(3), 161-3.

Finlay, B.L., \& Slattery, M. (1983). Local differences in the amount of early cell death in neocortex predict adult local specializations. Science, 219(4590), 1349-51.

Fox, N.L., Sexton, M., \& Hebel, J.R. (1990). Prenatal exposure to tobacco: I. Effects on physical growth at age three. Int J Epidemiol, 19(1), 66-71.

Frezza, M., di Padova, C., Pozzato, G., Terpin, M., Baraona, E., \& Lieber, C.S. (1990). High blood alcohol levels in women. The role of decreased gastric alcohol dehydrogenase activity and first-pass metabolism. $N$ Engl J Med, 322(2), 95-9.

Friedman, B., \& Price, J.L. (1986). Age-dependent cell death in the olfactory cortex: lack of transneuronal degeneration in neonates. J Comp Neurol, 246(1), 20-31.

Galofre, E., Ferrer, I., Fabregues, I., \& Lopez-Tejero, D. (1987). Effects of prenatal ethanol exposure on dendritic spines of layer $\mathrm{V}$ pyramidal neurons in the somatosensory cortex of the rat. J Neurol Sci, 81(2-3), 185-95.

Gharbawie, O.A., Gonzalez, C.L., Williams, P.T., Kleim, J.A., \& Whishaw, I.Q. (2005). Middle cerebral artery (MCA) stroke produces dysfunction in adjacent motor cortex as detected by intracortical microstimulation in rats. Neuroscience, 130(3), 601-10.

Gibson, M.A., Butters, N.S., Reynolds, J.N., \& Brien, J.F. (2000). Effects of chronic prenatal ethanol exposure on locomotor activity, and hippocampal weight, neurons, and nitric oxide synthase activity of the young postnatal guinea pig. Neurotoxicol Teratol, 22(2), 183-92. 
Gil, O.D., Needleman, L., \& Huntley, G.W. (2002). Developmental patterns of cadherin expression and localization in relation to compartmentalized thalamocortical terminations in rat barrel cortex. J Comp Neurol, 453(4), 372-88.

Gioanni, Y., \& Lamarche, M. (1985). A reappraisal of rat motor cortex organization by intracortical microstimulation. Brain Res, 344(1), 49-61.

Globus, A., Rosenzweig, M.R., Bennett, E.L., \& Diamond, M.C. (1973). Effects of differential experience on dendritic spine counts in rat cerebral cortex. J Comp Physiol Psychol, 82(2), 175-81.

Gongidi, V., Ring, C., Moody, M., Brekken, R., Sage, E.H., Rakic, P., \& Anton, E.S. (2004). SPARC-like 1 regulates the terminal phase of radial glia-guided migration in the cerebral cortex. Neuron, 41(1), 57-69.

Goodlett, C.R., \& Horn, K.H. (2001). Mechanisms of alcohol-induced damage to the developing nervous system. Alcohol Res Health, 25(3), 175-84.

Goodlett, C.R., Horn, K.H., \& Zhou, F.C. (2005). Alcohol teratogenesis: mechanisms of damage and strategies for intervention. Exp Biol Med (Maywood), 230(6), 394406.

Goodlett, C.R., \& Lundahl, K.R. (1996). Temporal determinants of neonatal alcoholinduced cerebellar damage and motor performance deficits. Pharmacol Biochem Behav, 55(4), 531-40.

Granato, A. (2006). Altered organization of cortical interneurons in rats exposed to ethanol during neonatal life. Brain Res, 1069(1), 23-30.

Greenough, W.T., Hwang, H.M., \& Gorman, C. (1985). Evidence for active synapse formation or altered postsynaptic metabolism in visual cortex of rats reared in complex environments. Proc Natl Acad Sci U S A, 82(13), 4549-52.

Greenough, W.T., \& Volkmar, F.R. (1973). Pattern of dendritic branching in occipital cortex of rats reared in complex environments. Exp Neurol, 40(2), 491-504.

Greenough, W.T., Volkmar, F.R., \& Juraska, J.M. (1973). Effects of rearing complexity on dendritic branching in frontolateral and temporal cortex of the rat. Exp Neurol, 41(2), 371-8.

Guerri, C. (1998). Neuroanatomical and neurophysiological mechanisms involved in central nervous system dysfunctions induced by prenatal alcohol exposure. Alcohol Clin Exp Res, 22(2), 304-12.

Guerri, C., \& Renau-Piqueras, J. (1997). Alcohol, astroglia, and brain development. Mol Neurobiol, 15(1), 65-81.

Habbick, B.F., Blakley, P.M., Houston, C.S., Snyder, R.E., Senthilselvan, A., \& Nanson, J.L. (1998). Bone age and growth in fetal alcohol syndrome. Alcohol Clin Exp Res, 22(6), 1312-6.

Haidarliu, S., \& Ahissar, E. (2001). Size gradients of barreloids in the rat thalamus. $J$ Comp Neurol, 429(3), 372-87.

Hall, R.D., \& Lindholm, E.P. (1974). Organization of motor and somatosensory neocortex in the albino rat. Brain Res(66), 23-38.

Halliwell, B. (1987). Oxidants and human disease: some new concepts. Faseb J, 1(5), 358-64.

Hamilton, D.A., Kodituwakku, P., Sutherland, R.J., \& Savage, D.D. (2003). Children with Fetal Alcohol Syndrome are impaired at place learning but not cuednavigation in a virtual Morris water task. Behav Brain Res, 143(1), 85-94. 
Hannigan, J.H., Berman, R.F., \& Zajac, C.S. (1993). Environmental enrichment and the behavioral effects of prenatal exposure to alcohol in rats. Neurotoxicol Teratol, 15(4), 261-6.

Hannigan, J.H., \& Riley, E.P. (1988). Prenatal ethanol alters gait in rats. Alcohol, 5(6), 451-4.

Haorah, J., Knipe, B., Leibhart, J., Ghorpade, A., \& Persidsky, Y. (2005). Alcoholinduced oxidative stress in brain endothelial cells causes blood-brain barrier dysfunction. J Leukoc Biol, 78(6), 1223-32.

Heaton, M.B., Mitchell, J.J., \& Paiva, M. (2000). Amelioration of ethanol-induced neurotoxicity in the neonatal rat central nervous system by antioxidant therapy. Alcohol Clin Exp Res, 24(4), 512-8.

Heaton, M.B., Paiva, M., Madorsky, I., \& Shaw, G. (2003). Ethanol effects on neonatal rat cortex: comparative analyses of neurotrophic factors, apoptosis-related proteins, and oxidative processes during vulnerable and resistant periods. Brain Res Dev Brain Res, 145(2), 249-62.

Hebel, J.R., Fox, N.L., \& Sexton, M. (1988). Dose-response of birth weight to various measures of maternal smoking during pregnancy. J Clin Epidemiol, 41(5), 483-9.

Heumann, D., Leuba, G., \& Rabinowicz, T. (1978). Postnatal development of the mouse cerebral neocortex. IV. Evolution of the total cortical volume, of the population of neurons and glial cells. J Hirnforsch, 19(5), 385-93.

Hicks, S.P., \& D'Amato, C.J. (1968). Cell migrations to the isocortex in the rat. Anat Rec, 160(3), 619-34.

Hoogland, P.V., Welker, E., \& Van der Loos, H. (1987). Organization of the projections from barrel cortex to thalamus in mice studied with Phaseolus vulgarisleucoagglutinin and HRP. Exp Brain Res, 68(1), 73-87.

Horton, J.C., \& Adams, D.L. (2005). The cortical column: a structure without a function. Philos Trans R Soc Lond B Biol Sci, 360(1456), 837-62.

Hoyme, H.E., May, P.A., Kalberg, W.O., Kodituwakku, P., Gossage, J.P., Trujillo, P.M., Buckley, D.G., Miller, J.H., Aragon, A.S., Khaole, N., Viljoen, D.L., Jones, K.L., \& Robinson, L.K. (2005). A practical clinical approach to diagnosis of fetal alcohol spectrum disorders: clarification of the 1996 institute of medicine criteria. Pediatrics, 115(1), 39-47.

Hubel, D.H., Wiesel, T.N., \& Stryker, M.P. (1978). Anatomical demonstration of orientation columns in macaque monkey. J Comp Neurol, 177(3), 361-80.

Imig, T.J., \& Adrian, H.O. (1977). Binaural columns in the primary field (A1) of cat auditory cortex. Brain Res, 138(2), 241-57.

Imig, T.J., \& Brugge, J.F. (1978). Sources and terminations of callosal axons related to binaural and frequency maps in primary auditory cortex of the cat. J Comp Neurol, 182(4), 637-60.

Inomata, K., Nasu, F., \& Tanaka, H. (1987). Decreased density of synaptic formation in the frontal cortex of neonatal rats exposed to ethanol in utero. Int J Dev Neurosci, 5(5-6), 455-60.

Jacquin, M.F., Rhoades, R.W., \& Klein, B.G. (1995). Structure-function relationships in rat brainstem subnucleus interpolaris. XI. Effects of chronic whisker trimming from birth. J Comp Neurol, 356(2), 200-24. 
Jaffer, Z., Nelson, M., \& Beighton, P. (1981). Bone fusion in the foetal alcohol syndrome. J Bone Joint Surg Br, 63B(4), 569-71.

Janzen, L.A., Nanson, J.L., \& Block, G.W. (1995). Neuropsychological evaluation of preschoolers with fetal alcohol syndrome. Neurotoxicol Teratol, 17(3), 273-9.

Jensen, K.F., \& Killackey, H.P. (1987). Terminal arbors of axons projecting to the somatosensory cortex of the adult rat. II. The altered morphology of thalamocortical afferents following neonatal infraorbital nerve cut. J Neurosci, 7(11), 3544-53.

Jeret, J.S., Serur, D., Wisniewski, K., \& Fisch, C. (1985). Frequency of agenesis of the corpus callosum in the developmentally disabled population as determined by computerized tomography. Pediatr Neurosci, 12(2), 101-3.

Jeret, J.S., Serur, D., Wisniewski, K.E., \& Lubin, R.A. (1987). Clinicopathological findings associated with agenesis of the corpus callosum. Brain Dev, 9(3), 25564.

Johnson, C.S., Blanton, M.R., \& Hunter, E.S., 3rd (2004). Effects of ethanol and hydrogen peroxide on mouse limb bud mesenchyme differentiation and cell death. In Vitro Cell Dev Biol Anim, 40(3-4), 108-12.

Jones, K.L., \& Smith, D.W. (1973). Recognition of the fetal alcohol syndrome in early infancy. Lancet, 2(7836), 999-1001.

Jones, K.L., \& Smith, D.W. (1975). The fetal alcohol syndrome. Teratology, 12(1), 1-10.

Jones, K.L., Smith, D.W., Ulleland, C.N., \& Streissguth, P. (1973). Pattern of malformation in offspring of chronic alcoholic mothers. Lancet, 1(7815), 1267 71.

Jones, P.J., Leichter, J., \& Lee, M. (1981). Placental blood flow in rats fed alcohol before and during gestation. Life Sci, 29(11), 1153-9.

Kaas, J., \& Ebner, F. (1998). Intrathalamic connections: a new way to modulate cortical plasticity? Nat Neurosci, 1(5), 341-2.

Kaas, J.H., Nelson, R.J., Sur, M., Lin, C.S., \& Merzenich, M.M. (1979). Multiple representations of the body within the primary somatosensory cortex of primates. Science, 204(4392), 521-3.

Kable, J.A., \& Coles, C.D. (2004). The impact of prenatal alcohol exposure on neurophysiological encoding of environmental events at six months. Alcohol Clin Exp Res, 28(3), 489-96.

Kahkonen, S., Kesaniemi, M., Nikouline, V.V., Karhu, J., Ollikainen, M., Holi, M., \& Ilmoniemi, R.J. (2001). Ethanol modulates cortical activity: direct evidence with combined TMS and EEG. Neuroimage, 14(2), 322-8.

Kalberg, W.O., Provost, B., Tollison, S.J., Tabachnick, B.G., Robinson, L.K., Eugene Hoyme, H., Trujillo, P.M., Buckley, D., Aragon, A.S., \& May, P.A. (2006). Comparison of motor delays in young children with fetal alcohol syndrome to those with prenatal alcohol exposure and with no prenatal alcohol exposure. Alcohol Clin Exp Res, 30(12), 2037-45.

Kandel, E.R., \& Schwartz, J.H. (1985). Principles of Neural Science. New York: Elsevier Science Publishing Co.

Kanold, P.O., Kara, P., Reid, R.C., \& Shatz, C.J. (2003). Role of subplate neurons in functional maturation of visual cortical columns. Science, 301(5632), 521-5. 
Kaplan, M.S., \& Hinds, J.W. (1977). Neurogenesis in the adult rat: electron microscopic analysis of light radioautographs. Science, 197(4308), 1092-4.

Kaplan-Estrin, M., Jacobson, S.W., \& Jacobson, J.L. (1999). Neurobehavioral effects of prenatal alcohol exposure at 26 months. Neurotoxicol Teratol, 21(5), 503-11.

Kartje-Tillotson, G., Neafsey, E.J., \& Castro, A.J. (1985). Electrophysiological analysis of motor cortical plasticity after cortical lesions in newborn rats. Brain Res, 332(1), 103-11.

Kelly, S.J., Day, N., \& Streissguth, A.P. (2000). Effects of prenatal alcohol exposure on social behavior in humans and other species. Neurotoxicol Teratol, 22(2), 143-9.

Kelly, S.J., Hulsether, S.A., \& West, J.R. (1987). Alterations in sensorimotor development: relationship to postnatal alcohol exposure. Neurotoxicol Teratol, 9(3), 243-51.

Kerns, K.A., Don, A., Mateer, C.A., \& Streissguth, A.P. (1997). Cognitive deficits in nonretarded adults with fetal alcohol syndrome. J Learn Disabil, 30(6), 685-93.

Killackey, H.P. (1973). Anatomical evidence for cortical subdivisions based on vertically discrete thalamic projections from the ventral posterior nucleus to cortical barrels in the rat. Brain Res, 51, 326-31.

Kim, U., \& Ebner, F.F. (1999). Barrels and septa: separate circuits in rat barrels field cortex. J Comp Neurol, 408(4), 489-505.

Klug, M.G., Burd, L., Martsolf, J.T., \& Ebertowski, M. (2003). Body mass index in fetal alcohol syndrome. Neurotoxicol Teratol, 25(6), 689-96.

Kopera-Frye, K., Dehaene, S., \& Streissguth, A.P. (1996). Impairments of number processing induced by prenatal alcohol exposure. Neuropsychologia, 34(12), 1187-96.

Koralek, K.A., Jensen, K.F., \& Killackey, H.P. (1988). Evidence for two complementary patterns of thalamic input to the rat somatosensory cortex. Brain Res, 463(2), 34651.

Kossut, M., \& Juliano, S.L. (1999). Anatomical correlates of representational map reorganization induced by partial vibrissectomy in the barrel cortex of adult mice. Neuroscience, 92(3), 807-17.

Kotch, L.E., Chen, S.Y., \& Sulik, K.K. (1995). Ethanol-induced teratogenesis: free radical damage as a possible mechanism. Teratology, 52(3), 128-36.

Kotch, L.E., Dehart, D.B., Alles, A.J., Chernoff, N., \& Sulik, K.K. (1992). Pathogenesis of ethanol-induced limb reduction defects in mice. Teratology, 46(4), 323-32.

Kotch, L.E., \& Sulik, K.K. (1992). Patterns of ethanol-induced cell death in the developing nervous system of mice; neural fold states through the time of anterior neural tube closure. Int J Dev Neurosci, 10(4), 273-9.

Kotkoskie, L.A., \& Norton, S. (1989). Cerebral cortical morphology and behavior in rats following acute prenatal ethanol exposure. Alcohol Clin Exp Res, 13(6), 776-81.

Kroemer, G., Zamzami, N., \& Susin, S.A. (1997). Mitochondrial control of apoptosis. Immunol Today, 18(1), 44-51.

Kumral, A., Tugyan, K., Gonenc, S., Genc, K., Genc, S., Sonmez, U., Yilmaz, O., Duman, N., Uysal, N., \& Ozkan, H. (2005). Protective effects of erythropoietin against ethanol-induced apoptotic neurodegenaration and oxidative stress in the developing C57BL/6 mouse brain. Brain Res Dev Brain Res, 160(2), 146-56. 
Lamarche, F., Gonthier, B., Signorini, N., Eysseric, H., \& Barret, L. (2003). Acute exposure of cultured neurones to ethanol results in reversible DNA single-strand breaks; whereas chronic exposure causes loss of cell viability. Alcohol and Alcoholism, 38(6), 550-8.

Lancaster, F.E., Phillips, S.M., Patsalos, P.N., \& Wiggins, R.C. (1984). Brain myelination in the offspring of ethanol-treated rats: in utero versus lactational exposure by crossfostering offspring of control, pairfed and ethanol treated dams. Brain Res, 309(2), 209-16.

Land, P.W., Buffer, S.A., Jr., \& Yaskosky, J.D. (1995). Barreloids in adult rat thalamus: three-dimensional architecture and relationship to somatosensory cortical barrels. J Comp Neurol, 355(4), 573-88.

Landers, M., \& Philip Zeigler, H. (2006). Development of rodent whisking: trigeminal input and central pattern generation. Somatosens Mot Res, 23(1-2), 1-10.

Larry, S.R., James, R.L., Nicholas, S.C., Michael, Z.J., \& Susan, M.K. (2003). Fundamental Neuroscience. San Diego, California: Academic Press.

Lee, K.J., \& Woolsey, T.A. (1975). A proportional relationship between peripheral innervation density and cortical neuron number in the somatosensory system of the mouse. Brain Res, 99(2), 349-53.

Lemoine, P. (1992). [Outcome of children of alcoholic mothers (study of 105 cases followed to adult age) and various prophylactic findings]. Ann Pediatr (Paris), 39(4), 226-35.

LeVay, S., Hubel, D.H., \& Wiesel, T.N. (1975). The pattern of ocular dominance columns in macaque visual cortex revealed by a reduced silver stain. J Comp Neurol, 159(4), 559-76.

LeVay, S., Wiesel, T.N., \& Hubel, D.H. (1980). The development of ocular dominance columns in normal and visually deprived monkeys. J Comp Neurol, 191(1), 1-51.

Li, C.X., \& Waters, R.S. (1991). Organization of the mouse motor cortex studied by retrograde tracing and intracortical microstimulation (ICMS) mapping. Can J Neurol Sci, 18(1), 28-38.

Li, C.X., Waters, R.S., McCandlish, C.A., \& Johnson, E.F. (1996). Electrical stimulation of a forepaw digit increases the physiological representation of that digit in layer IV of SI cortex in rat. Neuroreport, 7(14), 2395-400.

Lieber, C.S. (1997). Cytochrome P-4502E1: its physiological and pathological role. Physiol Rev, 77(2), 517-44.

Lin, C.S., Merzenich, M.M., Sur, M., \& Kaas, J.H. (1979). Connections of areas 3b and 1 of the parietal somatosensory strip with the ventroposterior nucleus in the owl monkey (Aotus trivirgatus). J Comp Neurol, 185(2), 355-71.

Lindblad, B., \& Olsson, R. (1976). Unusually high levels of blood alcohol? Jama, 236(14), 1600-2.

Livy, D.J., \& Elberger, A.J. (2001). Effect of prenatal alcohol exposure on midsagittal commissure size in rats. Teratology, 63(1), 15-22.

Livy, D.J., Maier, Se, \& West, J.R. (2001). Fetal alcohol exposure and temporal vulnerability: effects of binge-like alcohol exposure on the ventrolateral nucleus of the thalamus. Alcohol Clin Exp Res, 25(5), 774-80.

Lopez-Bendito, G., \& Molnar, Z. (2003). Thalamocortical development: how are we going to get there? Nat Rev Neurosci, 4(4), 276-89. 
Lopez-Tejero, D., Ferrer, I., M, L.L., \& Herrera, E. (1986). Effects of prenatal ethanol exposure on physical growth, sensory reflex maturation and brain development in the rat. Neuropathol Appl Neurobiol, 12(3), 251-60.

Lorente de No, R. (1922). La corteza cerebral del raton. Trab. Lab. Invest. Biol. (Madrid), 20, 41-78.

Lu, S.M., \& Lin, R.C. (1993). Thalamic afferents of the rat barrel cortex: a light- and electron-microscopic study using Phaseolus vulgaris leucoagglutinin as an anterograde tracer. Somatosens Mot Res, 10(1), 1-16.

Lubke, J., Egger, V., Sakmann, B., \& Feldmeyer, D. (2000). Columnar organization of dendrites and axons of single and synaptically coupled excitatory spiny neurons in layer 4 of the rat barrel cortex. J Neurosci, 20(14), 5300-11.

Lubke, J., Roth, A., Feldmeyer, D., \& Sakmann, B. (2003). Morphometric analysis of the columnar innervation domain of neurons connecting layer 4 and layer $2 / 3$ of juvenile rat barrel cortex. Cereb Cortex, 13(10), 1051-63.

Ma, P.M. (1993). Barrelettes--architectonic vibrissal representations in the brainstem trigeminal complex of the mouse. II. Normal post-natal development. J Comp Neurol, 327(3), 376-97.

Machin, R., Blasco, B., Bjugn, R., \& Avendano, C. (2004). The size of the whisker barrel field in adult rats: minimal nondirectional asymmetry and limited modifiability by chronic changes of the sensory input. Brain Res, 1025(1-2), 130-8.

Maier, D.L., Grieb, G.M., Stelzner, D.J., \& McCasland, J.S. (2003). Large-scale plasticity in barrel cortex following repeated whisker trimming in young adult hamsters. Exp Neurol, 184(2), 737-45.

Maier, S.E., Chen, W.J., Miller, J.A., \& West, J.R. (1997). Fetal alcohol exposure and temporal vulnerability regional differences in alcohol-induced microencephaly as a function of the timing of binge-like alcohol exposure during rat brain development. Alcohol Clin Exp Res, 21(8), 1418-28.

Maier, S.E., Miller, J.A., Blackwell, J.M., \& West, J.R. (1999). Fetal alcohol exposure and temporal vulnerability: regional differences in cell loss as a function of the timing of binge-like alcohol exposure during brain development. Alcohol Clin Exp Res, 23(4), 726-34.

Maier, S.E., Miller, J.A., \& West, J.R. (1999). Prenatal binge-like alcohol exposure in the rat results in region-specific deficits in brain growth. Neurotoxicol Teratol, 21(3), 285-91.

Maier, S.E., \& West, J.R. (2001a). Drinking patterns and alcohol-related birth defects. Alcohol Res Health, 25(3), 168-74.

Maier, S.E., \& West, J.R. (2001b). Regional differences in cell loss associated with binge-like alcohol exposure during the first two trimesters equivalent in the rat. Alcohol, 23(1), 49-57.

Maier, S.E., \& West, J.R. (2003). Alcohol and nutritional control treatments during neurogenesis in rat brain reduce total neuron number in locus coeruleus, but not in cerebellum or inferior olive. Alcohol, 30(1), 67-74.

Manger, P.R., Rosa, M.G., \& Collins, R. (2001). Somatotopic organization and cortical projections of the ventrobasal complex of the flying fox: an "inverted" wing representation in the thalamus. Somatosens Mot Res, 18(1), 19-30. 
Margret, C.P., Chappell, T.D., Li, C.X., Jan, T.A., Matta, S.G., Elberger, A.J., \& Waters, R.S. (2006a). Prenatal alcohol exposure (PAE) reduces the size of the forepaw representation in forepaw barrel subfield (FBS) cortex in neonatal rats: relationship between periphery and central representation. Exp Brain Res, 1-10. Margret, C.P., Elberger, A.J., Li, C.X., Chappell, T.D., Matta, S.G., \& Waters, R.S. (2005a). Prenatal alcohol exposure (PAE) delays thalamocortical input to barrel field cortex in early postnatal rats: A DiI labeling study. Alcohol Clin Exp Res, 29, $128 \mathrm{~A}$.

Margret, C.P., Li, C.X., Chappell, T.D., Elberger, A.J., Matta, S.G., \& Waters, R.S. (2006b). Prenatal alcohol exposure delays the development of the cortical barrel field in neonatal rats. Exp Brain Res, 172(1), 1-13.

Margret, C.P., Li, C.X., Elberger, A.J., Matta, S.G., Chappell, T.D., \& Waters, R.S. (2005b). Prenatal alcohol exposure alters the size, but not the pattern, of the whisker representation in neonatal rat barrel cortex. Exp Brain Res, 165(2), $167-$ 78.

Margret, C.P., Li, C.X., Matta, S.G., \& Waters, R.S. (2003). Prenatal alcohol exposure reduces the size of vibrissae and forepaw representations in neonate rat barrel field cortex without altering barrel pattern: relationship between peripheral structure and central representation. Alcohol Clin Exp Res, 27, 82A.

Margret, C.P., Li, C.X., Oladehin, A., Maier, S.E., \& Waters, R.S. (2006c). Effect of postnatal or third trimester equivalent exposure of alcohol on developing rodent barrel cortex. Alcohol Clin Exp Res, 30, 228A.

Marino, M.D., Aksenov, M.Y., \& Kelly, S.J. (2004). Vitamin E protects against alcoholinduced cell loss and oxidative stress in the neonatal rat hippocampus. Int J Dev Neurosci, 22(5-6), 363-77.

Mattson, S.N., Goodman, A.M., Caine, C., Delis, D.C., \& Riley, E.P. (1999). Executive functioning in children with heavy prenatal alcohol exposure. Alcohol Clin Exp Res, 23(11), 1808-15.

Mattson, S.N., \& Riley, E.P. (1999). Implicit and explicit memory functioning in children with heavy prenatal alcohol exposure. J Int Neuropsychol Soc, 5(5), 462-71.

Mattson, S.N., Riley, E.P., Gramling, L., Delis, D.C., \& Jones, K.L. (1997). Heavy prenatal alcohol exposure with or without physical features of fetal alcohol syndrome leads to IQ deficits. J Pediatr, 131(5), 718-21.

Mattson, S.N., Riley, E.P., Gramling, L., Delis, D.C., \& Jones, K.L. (1998). Neuropsychological comparison of alcohol-exposed children with or without physical features of fetal alcohol syndrome. Neuropsychology, 12(1), 146-53.

Mattson, S.N., Riley, E.P., Jernigan, T.L., Garcia, A., Kaneko, W.M., Ehlers, C.L., \& Jones, K.L. (1994). A decrease in the size of the basal ganglia following prenatal alcohol exposure: a preliminary report. Neurotoxicol Teratol, 16(3), 283-9.

Mattson, S.N., Riley, E.P., Sowell, E.R., Jernigan, T.L., Sobel, D.F., \& Jones, K.L. (1996). A decrease in the size of the basal ganglia in children with fetal alcohol syndrome. Alcohol Clin Exp Res, 20(6), 1088-93.

May, P.A., Brooke, L., Gossage, J.P., Croxford, J., Adnams, C., Jones, K.L., Robinson, L., \& Viljoen, D. (2000). Epidemiology of fetal alcohol syndrome in a South African community in the Western Cape Province. Am J Public Health, 90(12), 1905-12. 
May, P.A., \& Gossage, J.P. (2001). Estimating the prevalence of fetal alcohol syndrome. A summary. Alcohol Res Health, 25(3), 159-67.

May, P.A., Gossage, J.P., Brooke, L.E., Snell, C.L., Marais, A.S., Hendricks, L.S., Croxford, J.A., \& Viljoen, D.L. (2005). Maternal risk factors for fetal alcohol syndrome in the Western cape province of South Africa: a population-based study. Am J Public Health, 95(7), 1190-9.

May, P.A., Gossage, J.P., White-Country, M., Goodhart, K., Decoteau, S., Trujillo, P.M., Kalberg, W.O., Viljoen, D.L., \& Hoyme, H.E. (2004). Alcohol consumption and other maternal risk factors for fetal alcohol syndrome among three distinct samples of women before, during, and after pregnancy: the risk is relative. Am J Med Genet C Semin Med Genet, 127(1), 10-20.

McCandlish, C., Waters, R.S., \& Cooper, N.G. (1989). Early development of the representation of the body surface in SI cortex barrel field in neonatal rats as demonstrated with peanut agglutinin binding: evidence for differential development within the rattunculus. Exp Brain Res, 77(2), 425-31.

McCandlish, C.A., Li, C.X., \& Waters, R.S. (1993). Early development of the SI cortical barrel field representation in neonatal rats follows a lateral-to-medial gradient: an electrophysiological study. Exp Brain Res, 92(3), 369-74.

McCasland, J.S., Carvell, G.E., Simons, D.J., \& Woolsey, T.A. (1991). Functional asymmetries in the rodent barrel cortex. Somatosens Mot Res, 8(2), 111-6.

McCasland, J.S., \& Woolsey, T.A. (1988). High-resolution 2-deoxyglucose mapping of functional cortical columns in mouse barrel cortex. J Comp Neurol, 278(4), 55569.

McMechan, A.P., O'Leary-Moore, S.K., Morrison, S.D., \& Hannigan, J.H. (2004). Effects of prenatal alcohol exposure on forepaw digit length and digit ratios in rats. Dev Psychobiol, 45(4), 251-8.

Medina, A.E., Krahe, T.E., \& Ramoa, A.S. (2005). Early alcohol exposure induces persistent alteration of cortical columnar organization and reduced orientation selectivity in the visual cortex. J Neurophysiol, 93(3), 1317-25.

Melcer, T., Gonzalez, D., \& Riley, E.P. (1995). Locomotor activity and alcohol preference in alcohol-preferring and -nonpreferring rats following neonatal alcohol exposure. Neurotoxicol Teratol, 17(1), 41-8.

Melcer, T., Gonzalez, D., Somes, C., \& Riley, E.P. (1995). Neonatal alcohol exposure and early development of motor skills in alcohol preferring and nonpreferring rats. Neurotoxicol Teratol, 17(2), 103-10.

Melzer, P., \& Smith, C.B. (1998a). Plasticity of cerebral metabolic whisker maps in adult mice after whisker follicle removal--I. Modifications in barrel cortex coincide with reorganization of follicular innervation. Neuroscience, 83(1), 27-41.

Melzer, P., \& Smith, C.B. (1998b). Plasticity of cerebral metabolic whisker maps in adult mice after whisker follicle removal--II. Modifications in the subcortical somatosensory system. Neuroscience, 83(1), 43-61.

Merzenich, M.M., Kaas, J.H., Wall, J., Nelson, R.J., Sur, M., \& Felleman, D. (1983). Topographic reorganization of somatosensory cortical areas $3 \mathrm{~b}$ and 1 in adult monkeys following restricted deafferentation. Neuroscience, 8(1), 33-55. 
Metin, C., Baudoin, J.P., Rakic, S., \& Parnavelas, J.G. (2006). Cell and molecular mechanisms involved in the migration of cortical interneurons. Eur J Neurosci, 23(4), 894-900.

Meyer, L.S., Kotch, L.E., \& Riley, E.P. (1990). Alterations in gait following ethanol exposure during the brain growth spurt in rats. Alcohol Clin Exp Res, 14(1), 23-7.

Mierau, S.B., Meredith, R.M., Upton, A.L., \& Paulsen, O. (2004). Dissociation of experience-dependent and -independent changes in excitatory synaptic transmission during development of barrel cortex. Proc Natl Acad Sci U S A, 101(43), 15518-23.

Miller, M.W. (1986). Effects of alcohol on the generation and migration of cerebral cortical neurons. Science, 233(4770), 1308-11.

Miller, M.W. (1987). Effect of prenatal exposure to alcohol on the distribution and time of origin of corticospinal neurons in the rat. J Comp Neurol, 257(3), 372-82.

Miller, M.W. (1988). Effect of prenatal exposure to ethanol on the development of cerebral cortex: I. Neuronal generation. Alcohol Clin Exp Res, 12(3), 440-9.

Miller, M.W. (1989). Effects of prenatal exposure to ethanol on neocortical development: II. Cell proliferation in the ventricular and subventricular zones of the rat. J Comp Neurol, 287(3), 326-38.

Miller, M.W. (1993). Migration of cortical neurons is altered by gestational exposure to ethanol. Alcohol Clin Exp Res, 17(2), 304-14.

Miller, M.W. (1997). Effects of prenatal exposure to ethanol on callosal projection neurons in rat somatosensory cortex. Brain Res, 766(1-2), 121-8.

Miller, M.W. (2004). Repeated episodic exposure to ethanol affects neurotrophin content in the forebrain of the mature rat. Exp Neurol, 189(1), 173-81.

Miller, M.W. (2006). Effect of prenatal exposure to ethanol on glutamate and GABA immunoreactivity in macaque somatosensory and motor cortices: critical timing of exposure. Neuroscience, 138(1), 97-107.

Miller, M.W., Chiaia, N.L., \& Rhoades, R.W. (1990). Intracellular recording and injection study of corticospinal neurons in the rat somatosensory cortex: effect of prenatal exposure to ethanol. J Comp Neurol, 297(1), 91-105.

Miller, M.W., \& Dow-Edwards, D.L. (1988). Structural and metabolic alterations in rat cerebral cortex induced by prenatal exposure to ethanol. Brain Res, 474(2), 31626.

Miller, M.W., \& Dow-Edwards, D.L. (1993). Vibrissal stimulation affects glucose utilization in the trigeminal/somatosensory system of normal rats and rats prenatally exposed to ethanol. J Comp Neurol, 335(2), 283-4.

Miller, M.W., \& Potempa, G. (1990). Numbers of neurons and glia in mature rat somatosensory cortex: effects of prenatal exposure to ethanol. J Comp Neurol, 293(1), 92-102.

Minana, R., Climent, E., Barettino, D., Segui, J.M., Renau-Piqueras, J., \& Guerri, C. (2000). Alcohol exposure alters the expression pattern of neural cell adhesion molecules during brain development. J Neurochem, 75(3), 954-64.

Minciacchi, D., Granato, A., Santarelli, M., \& Sbriccoli, A. (1993). Modifications of thalamo-cortical circuitry in rats prenatally exposed to ethanol. Neuroreport, 4(4), 415-8. 
Miyashita, E., Keller, A., \& Asanuma, H. (1994). Input-output organization of the rat vibrissal motor cortex. Exp Brain Res, 99(2), 223-32.

Molina, J.C., Hoffmann, H., Spear, L.P., \& Spear, N.E. (1987). Sensorimotor maturation and alcohol responsiveness in rats prenatally exposed to alcohol during gestational day 8. Neurotoxicol Teratol, 9(2), 121-8.

Molnar, Z., Higashi, S., \& Lopez-Bendito, G. (2003). Choreography of early thalamocortical development. Cereb Cortex, 13(6), 661-9.

Montoliu, C., Sancho-Tello, M., Azorin, I., Burgal, M., Valles, S., Renau-Piqueras, J., \& Guerri, C. (1995). Ethanol increases cytochrome P4502E1 and induces oxidative stress in astrocytes. $J$ Neurochem, 65(6), 2561-70.

Montoliu, C., Valles, S., Renau-Piqueras, J., \& Guerri, C. (1994). Ethanol-induced oxygen radical formation and lipid peroxidation in rat brain: effect of chronic alcohol consumption. $J$ Neurochem, 63(5), 1855-62.

Mooney, S.M., \& Miller, M.W. (1999). Effects of prenatal exposure to ethanol on systems matching: the number of neurons in the ventrobasal thalamic nucleus of the mature rat. Brain Res Dev Brain Res, 117(1), 121-5.

Mooney, S.M., \& Miller, M.W. (2003). Ethanol-induced neuronal death in organotypic cultures of rat cerebral cortex. Brain Res Dev Brain Res, 147(1-2), 135-41.

Mooney, S.M., Napper, R.M., \& West, J.R. (1996). Long-term effect of postnatal alcohol exposure on the number of cells in the neocortex of the rat: a stereological study. Alcohol Clin Exp Res, 20(4), 615-23.

Moore, D.B., Madorsky, I., Paiva, M., \& Barrow Heaton, M. (2004). Ethanol exposure alters neurotrophin receptor expression in the rat central nervous system: Effects of prenatal exposure. J Neurobiol, 60(1), 101-13.

Mosconi, T.M., \& Rice, F.L. (1993). Sequential differentiation of sensory innervation in the mystacial pad of the ferret. J Comp Neurol, 333(3), 309-25.

Mountcastle, A. (1997a). Reframing refugees: the power of Tibetan identity. Coll Antropol, 21(2), 585-93.

Mountcastle, V. (1978). An organizing principle for cerebral function: the unit module and the distributed system. Massachusetts: MIT Press.

Mountcastle, V.B. (1957). Modality and topographic properties of single neurons of cat's somatic sensory cortex. J Neurophysiol, 20(4), 408-34.

Mountcastle, V.B. (1958). Somatic functions of the nervous system. Annu Rev Physiol, 20, 471-508.

Mountcastle, V.B. (1997b). The columnar organization of the neocortex. Brain, 120 (Pt 4), 701-22.

Mountcastle, V.B., Davies, P.W., \& Berman, A.L. (1957). Response properties of neurons of cat's somatic sensory cortex to peripheral stimuli. J Neurophysiol, 20(4), 374-407.

Munger, B.L., \& Rice, F.L. (1986). Successive waves of differentiation of cutaneous afferents in rat mystacial skin. J Comp Neurol, 252(3), 404-14.

Nadarajah, B., \& Parnavelas, J.G. (2002). Modes of neuronal migration in the developing cerebral cortex. Nat Rev Neurosci, 3(6), 423-32.

Nakano, T., Fujimoto, T., Shimooki, S., Fukudome, T., Uchida, T., Tsuji, T., Mitsuyama, Y., Akimoto, H., \& Furukawa, S. (1996). Transient elevation of nerve growth 
factor content in the rat hippocampus and frontal cortex by chronic ethanol treatment. Psychiatry Clin Neurosci, 50(3), 157-60.

Nanji, A.A., \& Hiller-Sturmhofel, S. (1997). Apoptosis and necrosis: two types of cell death in liver disease. Alcohol Health Res World, 21(4), 325-30.

Nanson, J.L., \& Hiscock, M. (1990). Attention deficits in children exposed to alcohol prenatally. Alcohol Clin Exp Res, 14(5), 656-61.

Neafsey, E.J., Bold, E.L., Haas, G., Hurley-Gius, K.M., Quirk, G., Sievert, C.F., \& Terreberry, R.R. (1986). The organization of the rat motor cortex: a microstimulation mapping study. Brain Res, 396(1), 77-96.

Neafsey, E.J., \& Sievert, C. (1982). A second forelimb motor area exists in rat frontal cortex. Brain Res, 232(1), 151-6.

Neimark, M.A., Andermann, M.L., Hopfield, J.J., \& Moore, C.I. (2003). Vibrissa resonance as a transduction mechanism for tactile encoding. $J$ Neurosci, 23(16), 6499-509.

Nelson, R.J., Sur, M., Felleman, D.J., \& Kaas, J.H. (1980). Representations of the body surface in postcentral parietal cortex of Macaca fascicularis. J Comp Neurol, 192(4), 611-43.

NIAAA (1993). NIAAA (National Institue on Alcohol Abuse and Alcoholism). Eighth Special Report to the U.S. Congress on Alcohol and Health., NIAAA (National Institue on Alcohol Abuse and Alcoholism). Eighth Special Report to the U.S. Congress on Alcohol and Health. Washington, DC: U.S. Department of Health and Human Services.

Nithianantharajah, J., \& Hannan, A.J. (2006). Enriched environments, experiencedependent plasticity and disorders of the nervous system. Nat Rev Neurosci, 7(9), 697-709.

Nordstrom-Klee, B., Delaney-Black, V., Covington, C., Ager, J., \& Sokol, R. (2002). Growth from birth onwards of children prenatally exposed to drugs: a literature review. Neurotoxicol Teratol, 24(4), 481-8.

Norton, S., Terranova, P., Na, J.Y., \& Sancho-Tello, M. (1988). Early motor development and cerebral cortical morphology in rats exposed perinatally to alcohol. Alcohol Clin Exp Res, 12(1), 130-6.

Nurse, C.A., \& Farraway, L. (1988). Development of Merkel cell populations with contrasting sensitivities to neonatal deafferentation in the rat whisker pad. Somatosens Mot Res, 6(2), 141-62.

O'Hare, E.D., Kan, E., Yoshii, J., Mattson, S.N., Riley, E.P., Thompson, P.M., Toga, A.W., \& Sowell, E.R. (2005). Mapping cerebellar vermal morphology and cognitive correlates in prenatal alcohol exposure. Neuroreport, 16(12), 1285-90.

Olson, H.C., Feldman, J.J., Streissguth, A.P., Sampson, P.D., \& Bookstein, F.L. (1998). Neuropsychological deficits in adolescents with fetal alcohol syndrome: clinical findings. Alcohol Clin Exp Res, 22(9), 1998-2012.

Panzeri, S., Petroni, F., Petersen, R.S., \& Diamond, M.E. (2003). Decoding neuronal population activity in rat somatosensory cortex: role of columnar organization. Cereb Cortex, 13(1), 45-52.

Parnavelas, J.G. (2002). The origin of cortical neurons. Braz J Med Biol Res, 35(12), $1423-9$. 
Pauli, R.M., \& Feldman, P.F. (1986). Major limb malformations following intrauterine exposure to ethanol: two additional cases and literature review. Teratology, 33(3), 273-80.

Pearson, P.P., Arnold, P.B., Oladehin, A., Li, C.X., \& Waters, R.S. (2001). Large-scale cortical reorganization following forelimb deafferentation in rat does not involve plasticity of intracortical connections. Exp Brain Res, 138(1), 8-25.

Pearson, P.P., Li, C.X., Chappell, T.D., \& Waters, R.S. (2003). Delayed reorganization of the shoulder representation in forepaw barrel subfield (FBS) in first somatosensory cortex (SI) following forelimb deafferentation in adult rats. Exp Brain Res, 153(1), 100-12.

Pearson, P.P., Li, C.X., \& Waters, R.S. (1999). Effects of large-scale limb deafferentation on the morphological and physiological organization of the forepaw barrel subfield (FBS) in somatosensory cortex (SI) in adult and neonatal rats. Exp Brain Res, 128(3), 315-31.

Pearson, P.P., Oladehin, A., Li, C.X., Johnson, E.F., Weeden, A.M., Daniel, C.H., \& Waters, R.S. (1996). Relationship between representation of hindpaw and hindpaw barrel subfield (HBS) in layer IV of rat somatosensory cortex. Neuroreport, 7(14), 2317-23.

Penfield, W. (1972). The electrode, the brain and the mind. Z Neurol, 201(4), 297-309.

Penfield, W., \& Boldrey, E. (1937). Somatic motor and sensory representation in the cerebral cortex of man as studied by electrical stimulation. Brain, 60, 389-443.

Peng, Y., Kwok, K.H., Yang, P.H., Ng, S.S., Liu, J., Wong, O.G., He, M.L., Kung, H.F., \& Lin, M.C. (2005). Ascorbic acid inhibits ROS production, NF-kappaB activation and prevents ethanol-induced growth retardation and microencephaly. Neuropharmacology, 48(3), 426-434.

Petersen, C.C., Brecht, M., Hahn, T.T., \& Sakmann, B. (2004). Synaptic changes in layer $2 / 3$ underlying map plasticity of developing barrel cortex. Science, 304(5671), $739-42$.

Petersen, C.C., \& Sakmann, B. (2000). The excitatory neuronal network of rat layer 4 barrel cortex. J Neurosci, 20(20), 7579-86.

Polavarapu, R., Spitz, D.R., Sim, J.E., Follansbee, M.H., Oberley, L.W., Rahemtulla, A., \& Nanji, A.A. (1998). Increased lipid peroxidation and impaired antioxidant enzyme function is associated with pathological liver injury in experimental alcoholic liver disease in rats fed diets high in corn oil and fish oil. Hepatology, 27(5), 1317-23.

Popova, E.N. (1989). Ultrastructure of neurons of the sensorimotor cortex in progeny of rats receiving alcohol during pregnancy. Neurosci Behav Physiol, 19(5), 433-9.

Powrozek, T.A., \& Zhou, F.C. (2002). Effects of prenatal alcohol exposure on postnatal cortical barrel development.

Powrozek, T.A., \& Zhou, F.C. (2005). Effects of prenatal alcohol exposure on the development of the vibrissal somatosensory cortical barrel network. Brain Res Dev Brain Res, 155(2), 135-46.

Primatesta, P., Del Corno, G., Bonazzi, M.C., \& Waters, W.E. (1993). Alcohol and pregnancy: an international comparison. J Public Health Med, 15(1), 69-76.

Puddey, I.B., Zilkens, R.R., Croft, K.D., \& Beilin, L.J. (2001). Alcohol and endothelial function: a brief review. Clin Exp Pharmacol Physiol, 28(12), 1020-4. 
Pullarkat, R.K. (1991). Hypothesis: prenatal ethanol-induced birth defects and retinoic acid. Alcohol Clin Exp Res, 15(3), 565-7.

Qiang, M., Wang, M.W., \& Elberger, A.J. (2002). Second trimester prenatal alcohol exposure alters development of rat corpus callosum. Neurotoxicol Teratol, 24(6), 719-32.

Rakic, P. (1988). Specification of cerebral cortical areas. Science, 241(4862), 170-6.

Rakic, P. (2003). Developmental and evolutionary adaptations of cortical radial glia. Cereb Cortex, 13(6), 541-9.

Rakic, P., Bourgeois, J.P., Eckenhoff, M.F., Zecevic, N., \& Goldman-Rakic, P.S. (1986). Concurrent overproduction of synapses in diverse regions of the primate cerebral cortex. Science, 232(4747), 232-5.

Ramachandran, V., Perez, A., Chen, J., Senthil, D., Schenker, S., \& Henderson, G.I. (2001). In utero ethanol exposure causes mitochondrial dysfunction, which can result in apoptotic cell death in fetal brain: a potential role for 4-hydroxynonenal. Alcohol Clin Exp Res, 25(6), 862-71.

Ranck, J.B., Jr. (1975). Which elements are excited in electrical stimulation of mammalian central nervous system: a review. Brain Res, 98(3), 417-40.

Rao, D.N., Yang, M.X., Lasker, J.M., \& Cederbaum, A.I. (1996). 1-Hydroxyethyl radical formation during NADPH- and NADH-dependent oxidation of ethanol by human liver microsomes. Mol Pharmacol, 49(5), 814-21.

Reinke, L.A., Lai, E.K., DuBose, C.M., \& McCay, P.B. (1987). Reactive free radical generation in vivo in heart and liver of ethanol-fed rats: correlation with radical formation in vitro. Proc Natl Acad Sci U S A, 84(24), 9223-7.

Rema, V., Armstrong-James, M., \& Ebner, F.F. (2003). Experience-dependent plasticity is impaired in adult rat barrel cortex after whiskers are unused in early postnatal life. J Neurosci, 23(1), 358-66.

Rema, V., \& Ebner, F.F. (1999). Effect of enriched environment rearing on impairments in cortical excitability and plasticity after prenatal alcohol exposure. J Neurosci, 19(24), 10993-1006.

Renehan, W.E., \& Munger, B.L. (1986). Degeneration and regeneration of peripheral nerve in the rat trigeminal system. II. Response to nerve lesions. J Comp Neurol, 249(4), 429-59.

Rice, F.L., Mance, A., \& Munger, B.L. (1986). A comparative light microscopic analysis of the sensory innervation of the mystacial pad. I. Innervation of vibrissal folliclesinus complexes. J Comp Neurol, 252(2), 154-74.

Rice, F.L., \& Munger, B.L. (1986). A comparative light microscopic analysis of the sensory innervation of the mystacial pad. II. The common fur between the vibrissae. J Comp Neurol, 252(2), 186-205.

Rice, F.L., \& Van der Loos, H. (1977). Development of the barrels and barrel field in the somatosensory cortex of the mouse. J Comp Neurol, 171(4), 545-60.

Richardson, G.A., Ryan, C., Willford, J., Day, N.L., \& Goldschmidt, L. (2002). Prenatal alcohol and marijuana exposure: effects on neuropsychological outcomes at 10 years. Neurotoxicol Teratol, 24(3), 309-20.

Riddle, D., Richards, A., Zsuppan, F., \& Purves, D. (1992). Growth of the rat somatic sensory cortex and its constituent parts during postnatal development. J Neurosci, 12(9), 3509-24. 
Riddle, D.R., Gutierrez, G., Zheng, D., White, L.E., Richards, A., \& Purves, D. (1993). Differential metabolic and electrical activity in the somatic sensory cortex of juvenile and adult rats. $J$ Neurosci, 13(10), 4193-213.

Riikonen, R., Salonen, I., Partanen, K., \& Verho, S. (1999). Brain perfusion SPECT and MRI in foetal alcohol syndrome. Dev Med Child Neurol, 41(10), 652-9.

Riley, E.P., Barron, S., Melcer, T., \& Gonzalez, D. (1993). Alterations in activity following alcohol administration during the third trimester equivalent in $\mathrm{P}$ and $\mathrm{NP}$ rats. Alcohol Clin Exp Res, 17(6), 1240-6.

Riley, E.P., Mattson, S.N., Sowell, E.R., Jernigan, T.L., Sobel, D.F., \& Jones, K.L. (1995). Abnormalities of the corpus callosum in children prenatally exposed to alcohol. Alcohol Clin Exp Res, 19(5), 1198-202.

Riley, E.P., \& McGee, C.L. (2005). Fetal alcohol spectrum disorders: an overview with emphasis on changes in brain and behavior. Exp Biol Med (Maywood), 230(6), 357-65.

Riley, E.P., McGee, C.L., \& Sowell, E.R. (2004). Teratogenic effects of alcohol: a decade of brain imaging. Am J Med Genet C Semin Med Genet, 127(1), 35-41.

Rockland, K.S., \& Ichinohe, N. (2004). Some thoughts on cortical minicolumns. Exp Brain Res, 158(3), 265-77.

Roebuck, T.M., Mattson, S.N., \& Riley, E.P. (1998). A review of the neuroanatomical findings in children with fetal alcohol syndrome or prenatal exposure to alcohol. Alcohol Clin Exp Res, 22(2), 339-44.

Roebuck, T.M., Mattson, S.N., \& Riley, E.P. (2002). Interhemispheric transfer in children with heavy prenatal alcohol exposure. Alcohol Clin Exp Res, 26(12), 1863-71.

Roebuck, T.M., Simmons, R.W., Mattson, S.N., \& Riley, E.P. (1998a). Prenatal exposure to alcohol affects the ability to maintain postural balance. Alcohol Clin Exp Res, 22(1), 252-8.

Roebuck, T.M., Simmons, R.W., Richardson, C., Mattson, S.N., \& Riley, E.P. (1998b). Neuromuscular responses to disturbance of balance in children with prenatal exposure to alcohol. Alcohol Clin Exp Res, 22(9), 1992-7.

Roebuck-Spencer, T.M., Mattson, S.N., Marion, S.D., Brown, W.S., \& Riley, E.P. (2004). Bimanual coordination in alcohol-exposed children: role of the corpus callosum. J Int Neuropsychol Soc, 10(4), 536-48.

Rosen, I., \& Asanuma, H. (1972). Peripheral afferent inputs to the forelimb area of the monkey motor cortex: input-output relations. Exp Brain Res, 14(3), 257-73.

Rosett, H.L., Weiner, L., \& Edelin, K.C. (1981). Strategies for prevention of fetal alcohol effects. Obstet Gynecol, 57(1), 1-7.

Rosett, H.L., Weiner, L., Lee, A., Zuckerman, B., Dooling, E., \& Oppenheimer, E. (1983). Patterns of alcohol consumption and fetal development. Obstet Gynecol, 61(5), 539-46.

Sampson, P.D., Streissguth, A.P., Bookstein, F.L., Little, R.E., Clarren, S.K., Dehaene, P., Hanson, J.W., \& Graham, J.M., Jr. (1997). Incidence of fetal alcohol syndrome and prevalence of alcohol-related neurodevelopmental disorder. Teratology, 56(5), 317-26.

Sanes, J.N., \& Donoghue, J.P. (2000). Plasticity and primary motor cortex. Annu Rev Neurosci, 23, 393-415. 
Sanes, J.N., Suner, S., \& Donoghue, J.P. (1990). Dynamic organization of primary motor cortex output to target muscles in adult rats. I. Long-term patterns of reorganization following motor or mixed peripheral nerve lesions. Exp Brain Res, 79(3), 479-91.

Sari, Y., Powrozek, T., \& Zhou, F.C. (2001). Alcohol deters the outgrowth of serotonergic neurons at midgestation. J Biomed Sci, 8(1), 119-25.

Savage, D.D., Becher, M., de la Torre, A.J., \& Sutherland, R.J. (2002). Dose-dependent effects of prenatal ethanol exposure on synaptic plasticity and learning in mature offspring. Alcohol Clin Exp Res, 26(11), 1752-8.

Scheich, H. (1983). Two columnar systems in the auditory neostriatum of the chick: evidence from 2-deoxyglucose. Exp Brain Res, 51(2), 199-205.

Schlaggar, B.L., \& O'Leary, D.D. (1993). Patterning of the barrel field in somatosensory cortex with implications for the specification of neocortical areas. Perspect Dev Neurobiol, 1(2), 81-91.

Schlaggar, B.L., \& O'Leary, D.D. (1994). Early development of the somatotopic map and barrel patterning in rat somatosensory cortex. J Comp Neurol, 346(1), 80-96.

Senft, S.L., \& Woolsey, T.A. (1991). Growth of thalamic afferents into mouse barrel cortex. Cereb Cortex, 1(4), 308-35.

Seo, M.L. (1992). Effect of environmental complexity on the latency of cortical vibrissa potentials. Dev Psychobiol, 25(1), 67-76.

Sessler, F.M., Hsu, F.C., Felder, T.N., Zhai, J., Lin, R.C., Wieland, S.J., \& Kosobud, A.E. (1998). Effects of ethanol on rat somatosensory cortical neurons. Brain Res, 804(2), 266-74.

Shin, J.W., Lee, D.J., Jung, H.S., \& Sohn, N.W. (2005). Metabolic barrel representations with various patterns of neonatal whisker deafferentation in rats. Int $J$ Dev Neurosci, 23(6), 537-44.

Shoykhet, M., Land, P.W., \& Simons, D.J. (2005). Whisker trimming begun at birth or on postnatal day 12 affects excitatory and inhibitory receptive fields of layer IV barrel neurons. J Neurophysiol, 94(6), 3987-95.

Sievert, C.F., \& Neafsey, E.J. (1986). A chronic unit study of the sensory properties of neurons in the forelimb areas of rat sensorimotor cortex. Brain Res, 381(1), 15-23.

Simmons, R.W., Thomas, J.D., Levy, S.S., \& Riley, E.P. (2006). Motor response selection in children with fetal alcohol spectrum disorders. Neurotoxicol Teratol, 28(2), 278-85.

Simmons, R.W., Wass, T., Thomas, J.D., \& Riley, E.P. (2002). Fractionated simple and choice reaction time in children with prenatal exposure to alcohol. Alcohol Clin Exp Res, 26(9), 1412-9.

Simons, D.J. (1978). Response properties of vibrissa units in rat SI somatosensory neocortex. J Neurophysiol, 41(3), 798-820.

Singer, W. (1981). Topographic organization of orientation columns in the cat visual cortex. A deoxyglucose study. Exp Brain Res, 44(4), 431-6.

Singh, S.P., Snyder, A.K., \& Pullen, G.L. (1989). Maternal alcohol ingestion inhibits fetal glucose uptake and growth. Neurotoxicol Teratol, 11(3), 215-9.

Sirevaag, A.M., Black, J.E., Shafron, D., \& Greenough, W.T. (1988). Direct evidence that complex experience increases capillary branching and surface area in visual cortex of young rats. Brain Res, 471(2), 299-304. 
Sirevaag, A.M., \& Greenough, W.T. (1985). Differential rearing effects on rat visual cortex synapses. II. Synaptic morphometry. Brain Res, 351(2), 215-26.

Sirevaag, A.M., \& Greenough, W.T. (1988). A multivariate statistical summary of synaptic plasticity measures in rats exposed to complex, social and individual environments. Brain Res, 441(1-2), 386-92.

Slawecki, C.J. (2002). Altered EEG responses to ethanol in adult rats exposed to ethanol during adolescence. Alcohol Clin Exp Res, 26(2), 246-54.

Slawecki, C.J., Thomas, J.D., Riley, E.P., \& Ehlers, C.L. (2004). Neurophysiologic consequences of neonatal ethanol exposure in the rat. Alcohol, 34(2-3), 187-96.

Smart, I.H., \& McSherry, G.M. (1982). Growth patterns in the lateral wall of the mouse telencephalon. II. Histological changes during and subsequent to the period of isocortical neuron production. $J$ Anat, 134 (Pt 3), 415-42.

Sood, B., Delaney-Black, V., Covington, C., Nordstrom-Klee, B., Ager, J., Templin, T., Janisse, J., Martier, S., \& Sokol, R.J. (2001). Prenatal alcohol exposure and childhood behavior at age 6 to 7 years: I. dose-response effect. Pediatrics, 108(2), E34.

Sowell, E.R., Jernigan, T.L., Mattson, S.N., Riley, E.P., Sobel, D.F., \& Jones, K.L. (1996). Abnormal development of the cerebellar vermis in children prenatally exposed to alcohol: size reduction in lobules I-V. Alcohol Clin Exp Res, 20(1), 314.

Sowell, E.R., Thompson, P.M., Mattson, S.N., Tessner, K.D., Jernigan, T.L., Riley, E.P., \& Toga, A.W. (2001). Voxel-based morphometric analyses of the brain in children and adolescents prenatally exposed to alcohol. Neuroreport, 12(3), 51523.

Sowell, E.R., Thompson, P.M., Mattson, S.N., Tessner, K.D., Jernigan, T.L., Riley, E.P., \& Toga, A.W. (2002a). Regional brain shape abnormalities persist into adolescence after heavy prenatal alcohol exposure. Cereb Cortex, 12(8), 856-65.

Sowell, E.R., Thompson, P.M., Peterson, B.S., Mattson, S.N., Welcome, S.E., Henkenius, A.L., Riley, E.P., Jernigan, T.L., \& Toga, A.W. (2002b). Mapping cortical gray matter asymmetry patterns in adolescents with heavy prenatal alcohol exposure. Neuroimage, 17(4), 1807-19.

Spiegel, P.G., Pekman, W.M., Rich, B.H., Versteeg, C.N., Nelson, V., \& Dudnikov, M. (1979). The orthopedic aspects of the fetal alcohol syndrome. Clin Orthop Relat $\operatorname{Res}(139), 58-63$.

Staiger, J.F., Kotter, R., Zilles, K., \& Luhmann, H.J. (1999). Connectivity in the somatosensory cortex of the adolescent rat: an in vitro biocytin study. Anat Embryol (Berl), 199(4), 357-65.

Stettler, O., Tavitian, B., \& Moya, K.L. (1996). Differential synaptic vesicle protein expression in the barrel field of developing cortex. J Comp Neurol, 375(2), 32132.

Stratton, K., Howe, C., \& Battaglia, F. (1996). Fetal Alcohol Syndrome: Diagnosis, Epidemiology, Prevention and Treatment. Institute of Medicine. Washington, DC: National Academy Press.

Streissguth, A.P., Aase, J.M., Clarren, S.K., Randels, S.P., LaDue, R.A., \& Smith, D.F. (1991). Fetal alcohol syndrome in adolescents and adults. Jama, 265(15), 1961-7. 
Streissguth, A.P., Barr, H.M., \& Sampson, P.D. (1990). Moderate prenatal alcohol exposure: effects on child IQ and learning problems at age 7 1/2 years. Alcohol Clin Exp Res, 14(5), 662-9.

Streissguth, A.P., Bookstein, F.L., Sampson, P.D., \& Barr, H.M. (1989). Neurobehavioral effects of prenatal alcohol: Part III. PLS analyses of neuropsychologic tests. Neurotoxicol Teratol, 11(5), 493-507.

Stromland, K. (1985). Ocular abnormalities in the fetal alcohol syndrome. Acta Ophthalmol Suppl, 171, 1-50.

Stromland, K. (2004). Visual impairment and ocular abnormalities in children with fetal alcohol syndrome. Addict Biol, 9(2), 153-7; discussion 159-60.

Stromland, K., Mattson, S.N., Adnams, C., Autti-Ramo, I., Riley, E.P., \& Warren, K. (2005). Fetal Alcohol Spectrum Disorders: an International Perspective. Alcoholism: Clinical and Experimental Research, 29(6), 1121-1126.

Stuhmer, T., Puelles, L., Ekker, M., \& Rubenstein, J.L. (2002). Expression from a Dlx gene enhancer marks adult mouse cortical GABAergic neurons. Cereb Cortex, 12(1), 75-85.

Sun, A.Y., Ingelman-Sundberg, M., Neve, E., Matsumoto, H., Nishitani, Y., Minowa, Y., Fukui, Y., Bailey, S.M., Patel, V.B., Cunningham, C.C., Zima, T., Fialova, L., Mikulikova, L., Popov, P., Malbohan, I., Janebova, M., Nespor, K., \& Sun, G.Y. (2001). Ethanol and oxidative stress. Alcohol Clin Exp Res, 25(5 Suppl ISBRA), 237S-243S.

Sur, M., Nelson, R.J., \& Kaas, J.H. (1980). Representation of the body surface in somatic koniocortex in the prosimian Galago. J Comp Neurol, 189(2), 381-402.

Sweitzer, S.M., Fulk, L.J., Zhang, G., McKelvy, A., Bravo, M., \& Jinks, S.L. (2006). Neonatal Ethanol Exposure Produces Modality Dependent Changes in Nociception and Decreased Opiod Analgesia in Juvenile Rats: Behavioral and Neurophysiological Evidence., 228A-885.

Taylor, J.M., \& Crack, P.J. (2004). Impact of oxidative stress on neuronal survival. Clin Exp Pharmacol Physiol, 31(7), 397-406.

Tehovnik, E.J., Tolias, A.S., Sultan, F., Slocum, W.M., \& Logothetis, N.K. (2006). Direct and indirect activation of cortical neurons by electrical microstimulation. $J$ Neurophysiol, 96(2), 512-21.

Thomas, J.D., Burchette, T.L., Dominguez, H.D., \& Riley, E.P. (2000). Neonatal alcohol exposure produces more severe motor coordination deficits in high alcohol sensitive rats compared to low alcohol sensitive rats. Alcohol, 20(1), 93-9.

Thomas, J.D., Melcer, T., Weinert, S., \& Riley, E.P. (1998). Neonatal alcohol exposure produces hyperactivity in high-alcohol-sensitive but not in low-alcohol-sensitive rats. Alcohol, 16(3), 237-42.

Thomas, J.D., Wasserman, E.A., West, J.R., \& Goodlett, C.R. (1996). Behavioral deficits induced by bingelike exposure to alcohol in neonatal rats: importance of developmental timing and number of episodes. Dev Psychobiol, 29(5), 433-52.

Van der Loos, H., \& Woolsey, T.A. (1973). Somatosensory cortex: structural alterations following early injury to sense organs. Science, 179(71), 395-8.

Vierck, C.J., Jr., Favorov, O., \& Whitsel, B.L. (1988). Neural mechanisms of absolute tactile localization in monkeys. Somatosens Mot Res, 6(1), 41-61. 
Viljoen, D.L., Carr, L.G., Foroud, T.M., Brooke, L., Ramsay, M., \& Li, T.K. (2001). Alcohol dehydrogenase- $2 * 2$ allele is associated with decreased prevalence of fetal alcohol syndrome in the mixed-ancestry population of the Western Cape Province, South Africa. Alcohol Clin Exp Res, 25(12), 1719-22.

Wallace, M.N., \& Bajwa, S. (1991). Patchy intrinsic connections of the ferret primary auditory cortex. Neuroreport, 2(8), 417-20.

Walsh, R.N. (1981). Effects of environmental complexity and deprivation on brain anatomy and histology: a review. Int J Neurosci, 12(1), 33-51.

Warren, K.R., \& Li, T.K. (2005). Genetic polymorphisms: impact on the risk of fetal alcohol spectrum disorders. Birth Defects Res A Clin Mol Teratol, 73(4), 195-203.

Warren, R.K., \& Foudin, L.L. (2001). Alcohol-related Birth Defects - The Past, Present, and Future. Alcohol Research and Health, 25(3), 153-158.

Wass, T.S., Simmons, R.W., Thomas, J.D., \& Riley, E.P. (2002). Timing accuracy and variability in children with prenatal exposure to alcohol. Alcohol Clin Exp Res, 26(12), 1887-96.

Waters, R.S., Li, C.X., \& McCandlish, C.A. (1995). Relationship between the organization of the forepaw barrel subfield and the representation of the forepaw in layer IV of rat somatosensory cortex. Exp Brain Res, 103(2), 183-97.

Waters, R.S., McCandlish, C., \& Li, C.X. (1995). Organization and development of the forepaw representation in rodent barrel subfield (FBS) in somatosensory cortex of rat. In G. E. Jones \& I. T. Diamond (Eds.), Organization and development of the forepaw representation in rodent barrel subfield (FBS) in somatosensory cortex of rat. New York: Plenum Press.

Waters, R.S., McCandlish, C.A., \& Cooper, N.G. (1990). Early development of SI cortical barrel subfield representation of forelimb in normal and deafferented neonatal rat as delineated by peroxidase conjugated lectin, peanut agglutinin (PNA). Exp Brain Res, 81(2), 234-40.

Welker, C. (1976). Receptive fields of barrels in the somatosensory neocortex of the rat. $J$ Comp Neurol, 166(2), 173-89.

Welker, C., \& Woolsey, T.A. (1974). Structure of layer IV in the somatosensory neocortex of the rat: description and comparison with the mouse. J Comp Neurol, 158(4), 437-53.

Welker, E., Armstrong-James, M., Van der Loos, H., \& Kraftsik, R. (1993). The mode of activation of a barrel column: response properties of single units in the somatosensory cortex of the mouse upon whisker deflection. Eur J Neurosci, 5(6), 691-712.

Welker, E., \& Van der Loos, H. (1986). Quantitative correlation between barrel-field size and the sensory innervation of the whiskerpad: a comparative study in six strains of mice bred for different patterns of mystacial vibrissae. J Neurosci, 6(11), 335573.

Welker, W.I., Benjamin, R.M., Miles, R.C., \& Woolsey, C.N. (1957). Motor effects of stimulation of cerebral cortex of squirrel monkey (Saimiri sciureus). $J$ Neurophysiol, 20(4), 347-64.

Welt, C., \& Steindler, D.A. (1977). Somatosensory cortical barrels and thalamic barreloids in reeler mutant mice. Neuroscience, 2(5), 755-66. 
West, J.R., Black, A.C., Jr., Reimann, P.C., \& Alkana, R.L. (1981). Polydactyly and polysyndactyly induced by prenatal exposure to ethanol. Teratology, 24(1), 13-8.

West, J.R., Goodlett, C.R., Bonthius, D.J., Hamre, K.M., \& Marcussen, B.L. (1990). Cell population depletion associated with fetal alcohol brain damage: mechanisms of BAC-dependent cell loss. Alcohol Clin Exp Res, 14(6), 813-8.

White, E.L. (1989). Cortical Circuits: synaptic organization of the cerebral cortex. Stucture, function and theory. 132-149. Boston: Birkhauser Publishing.

Wichterle, H., Garcia-Verdugo, J.M., Herrera, D.G., \& Alvarez-Buylla, A. (1999). Young neurons from medial ganglionic eminence disperse in adult and embryonic brain. Nat Neurosci, 2(5), 461-6.

Williams, M.N., Zahm, D.S., \& Jacquin, M.F. (1994). Differential foci and synaptic organization of the principal and spinal trigeminal projections to the thalamus in the rat. Eur J Neurosci, 6(3), 429-53.

Winer, J.A. (1984). The pyramidal neurons in layer III of cat primary auditory cortex (AI). J Comp Neurol, 229(4), 476-96.

Wong-Riley, M. (1979). Columnar cortico-cortical interconnections within the visual system of the squirrel and macaque monkeys. Brain Res, 162(2), 201-17.

Wong-Riley, M.T., \& Welt, C. (1980). Histochemical changes in cytochrome oxidase of cortical barrels after vibrissal removal in neonatal and adult mice. Proc Natl Acad Sci U S A, 77(4), 2333-7.

Woolsey, C.N. (1964). Cortical localization as defined by evoked potentials and electrical stimulation studies. Madison, WI: University of Wisconsin Press.

Woolsey, C.N., Marshall, W.H., \& Bard, P. (1942). Represenation of cutaneous tactile sensitivity in the cerebral cortex of the monkey as indicated by evoked potentials. Bulletin Johns Hopkins Hospital, 70, 399-441.

Woolsey, T.A., Dierker, M.L., \& Wann, D.F. (1975). Mouse SmI cortex: qualitative and quantitative classification of golgi-impregnated barrel neurons. Proc Natl Acad Sci US A, 72(6), 2165-9.

Woolsey, T.A., \& Van der Loos, H. (1970). The structural organization of layer IV in the somatosensory region (SI) of mouse cerebral cortex. The description of a cortical field composed of discrete cytoarchitectonic units. Brain Res, 17(2), 205-42.

Woolsey, T.A., Welker, C., \& Schwartz, R.H. (1975). Comparative anatomical studies of the SmL face cortex with special reference to the occurrence of "barrels" in layer IV. J Comp Neurol, 164(1), 79-94.

Wu, D., \& Cederbaum, A.I. (2003). Alcohol, oxidative stress, and free radical damage. Alcohol Res Health, 27(4), 277-84.

Xerri, C., Coq, J.O., Merzenich, M.M., \& Jenkins, W.M. (1996). Experience-induced plasticity of cutaneous maps in the primary somatosensory cortex of adult monkeys and rats. $J$ Physiol Paris, 90(3-4), 277-87.

Zantua, J.B., Wasserstrom, S.P., Arends, J.J., Jacquin, M.F., \& Woolsey, T.A. (1996). Postnatal development of mouse "whisker" thalamus: ventroposterior medial nucleus (VPM), barreloids, and their thalamocortical relay neurons. Somatosens Mot Res, 13(3-4), 307-22.

Zhou, F.C., Bledsoe, S., Lumeng, L., \& Li, T.K. (1994a). Reduced serotonergic immunoreactive fibers in the forebrain of alcohol-preferring rats. Alcohol Clin Exp Res, 18(3), 571-9. 
Zhou, F.C., Pu, C.F., Murphy, J., Lumeng, L., \& Li, T.K. (1994b). Serotonergic neurons in the alcohol preferring rats. Alcohol, 11(5), 397-403.

Zhou, F.C., Sari, Y., Powrozek, T., Goodlett, C.R., \& Li, T.K. (2003). Moderate alcohol exposure compromises neural tube midline development in prenatal brain. Brain Res Dev Brain Res, 144(1), 43-55.

Zhou, F.C., Sari, Y., \& Powrozek, T.A. (2005). Fetal alcohol exposure reduces serotonin innervation and compromises development of the forebrain along the serotonergic pathway. Alcohol Clin Exp Res, 29(1), 141-9.

Zhou, F.C., Sari, Y., Zhang, J.K., Goodlett, C.R., \& Li, T. (2001). Prenatal alcohol exposure retards the migration and development of serotonin neurons in fetal C57BL mice. Brain Res Dev Brain Res, 126(2), 147-55.

Zima, T., Fialova, L., Mestek, O., Janebova, M., Crkovska, J., Malbohan, I., Stipek, S., Mikulikova, L., \& Popov, P. (2001). Oxidative stress, metabolism of ethanol and alcohol-related diseases. J Biomed Sci, 8(1), 59-70. 


\section{VITA}

Tyson Dow Chappell was born in West Valley City, Utah, on October 23, 1975, to Bill and Susan Chappell. Prior to graduating from Wayne High School in 1994, he had already begun his college education by taking courses from Snow College and Utah State University.

In August 1994 he matriculated into Weber State University in Ogden, Utah. After two years at Weber State, he postponed his education to serve for two years as a missionary for the Church of Jesus Christ of Latter Day Saints. During his mission he lived in Portugal and the islands of Cape Verde in West Africa.

After his mission, he returned to Weber State to receive his bachelors degree in psychology. In August 2001, he began his doctoral education at the University of Tennessee Health Science Center, Memphis, Tennessee. In December 2007 he received his Ph.D. degree in neuroscience for his study of " The long-term effects of prenatal alcohol exposure on the somatosensory and motor cortex of rats."

He currently lives with his wife, Amber Chappell, and their four children in Memphis, Tennessee. 